Prepared in cooperation with the Washington, D.C., Department of Energy \& Environment

\title{
Sediment and Chemical Contaminant Loads in Tributaries to the Anacostia River, Washington, District of Columbia, 2016-17
}

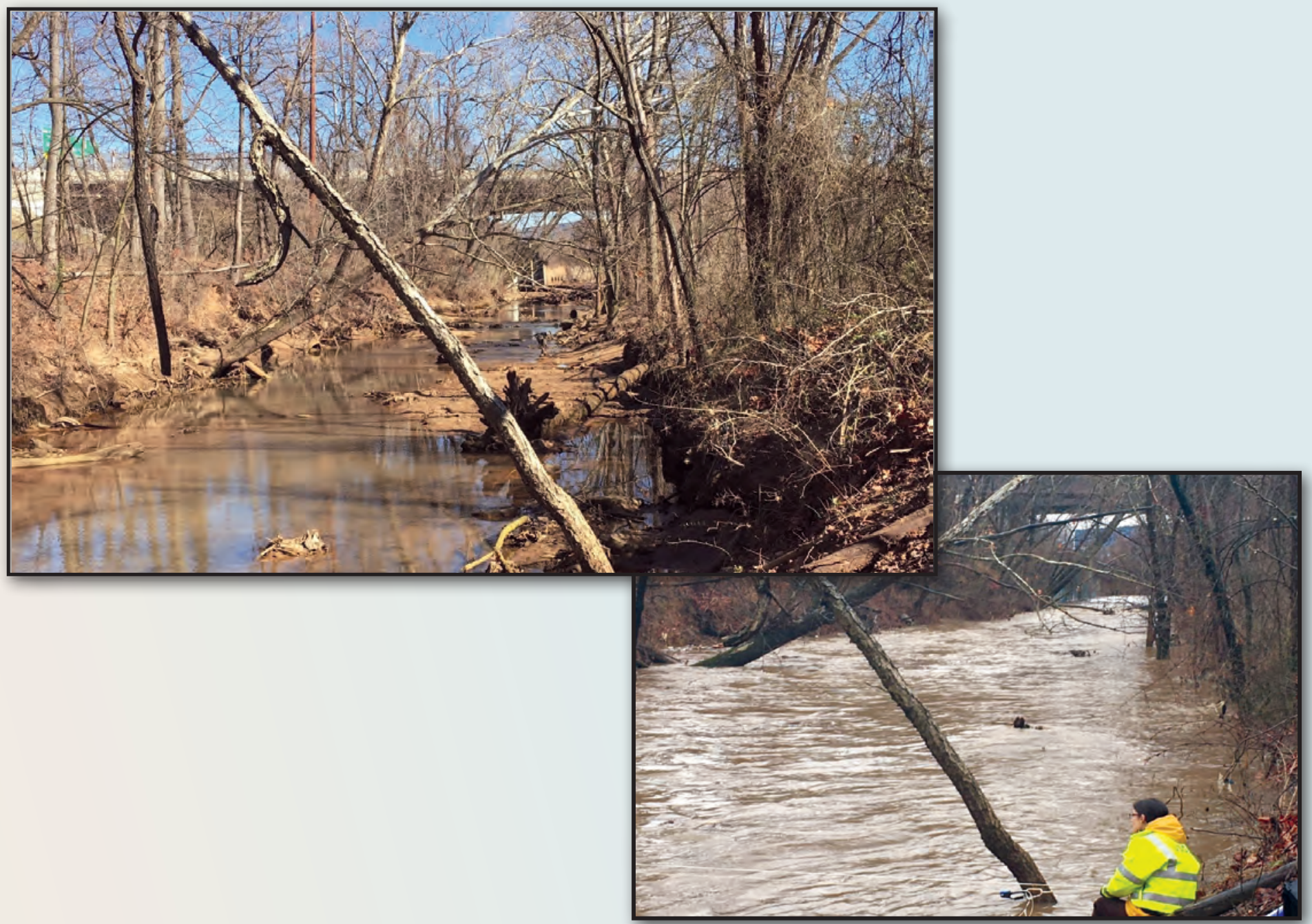

Scientific Investigations Report 2019-5092 
Cover. Low-flow (upper left, June 2017) and flood (lower right, March 2017) conditions at U.S. Geological Survey station 01651730, Beaverdam Creek near Cheverly, Maryland. Photographs by T.P. Wilson, U.S. Geological Survey. 


\section{Sediment and Chemical Contaminant Loads in Tributaries to the Anacostia River, Washington, District of Columbia, 2016-17}

By Timothy P. Wilson

Prepared in cooperation with the

Washington, D.C., Department of Energy \& Environment

Scientific Investigations Report 2019-5092 


\title{
U.S. Department of the Interior DAVID BERNHARDT, Secretary
}

\author{
U.S. Geological Survey \\ James F. Reilly II, Director
}

\section{U.S. Geological Survey, Reston, Virginia: 2019}

For more information on the USGS - the Federal source for science about the Earth, its natural and living resources, natural hazards, and the environment-visit https://www.usgs.gov or call 1-888-ASK-USGS.

For an overview of USGS information products, including maps, imagery, and publications, visit https://store.usgs.gov.

Any use of trade, firm, or product names is for descriptive purposes only and does not imply endorsement by the U.S. Government.

Although this information product, for the most part, is in the public domain, it also may contain copyrighted materials as noted in the text. Permission to reproduce copyrighted items must be secured from the copyright owner.

Suggested citation:

Wilson, T.P., 2019, Sediment and chemical contaminant loads in tributaries to the Anacostia River, Washington, District of Columbia, 2016-17: U.S. Geological Survey Scientific Investigations Report 2019-5092, 146 p., https://doi.org/10.3133/sir20195092.

Associated data for this publication:

Wilson, T.P., 2019, Discharge and sediment data for selected tributaries to the Anacostia River, Washington, District of Columbia, 2003-18: U.S. Geological Survey data release, https://doi.org/10.5066/P9RUZSMV. 


\section{Acknowledgments}

The author thanks Dev Murali of the Washington, D.C., Department of Energy \& Environment for help in planning and gaining access to sampling sites. The assistance of U.S. Geological Survey employees Charles Walker, Brenda Majedi, Brian Banks, Shannon Jackson, and Jeffrey Kvech in planning, aiding in fieldwork, and developing gaging and sampling stations is gratefully acknowledged. 


\section{Contents}

Acknowledgments .......................................................................................................................ii

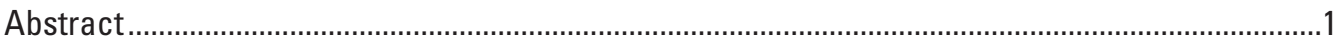

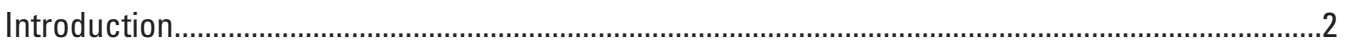

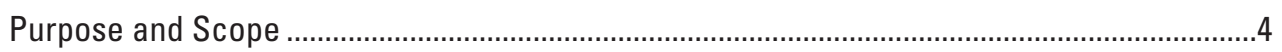

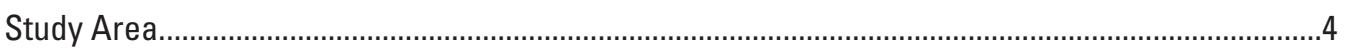

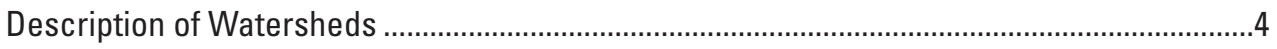

Methods

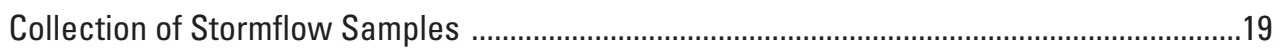

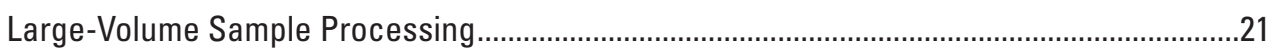

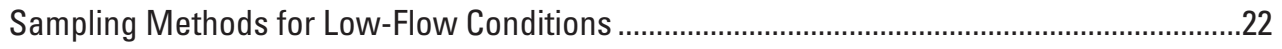

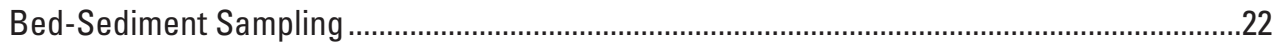

Sampling for Suspended Sediment and Particulate Organic Carbon.......................................22

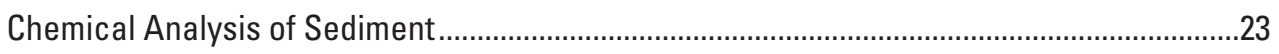

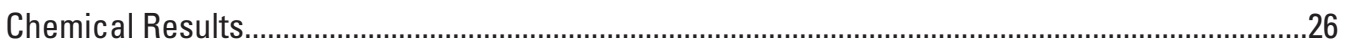

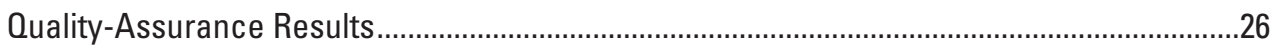

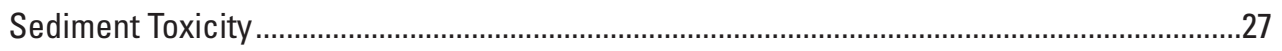

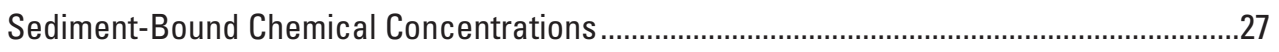

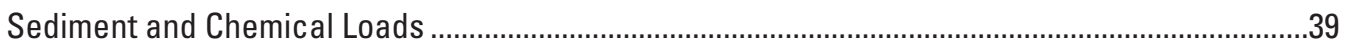

General Load Calculations ............................................................................................

Discharge in Gaged Tributaries.................................................................................

Discharge in Ungaged Tributaries ...............................................................................

Estimating Suspended-Sediment Concentrations from Measurements

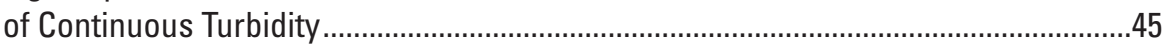

Sediment Loads in Gaged Tributaries.....................................................................

Sediment Loads in Ungaged Tributaries ..................................................................54

Concentrations of Sediment-Bound Contaminants ................................................................54

Representativeness of Samples.................................................................................58

Polychlorinated Biphenyls and Polycyclic Aromatic Hydrocarbons in Suspended Sediment and Bed Sediment.................................................................................59

Loads of Polychlorinated Biphenyls and Polycyclic Aromatic Hydrocarbons ........................79

Loads of Polychlorinated Biphenyls and Polyaromatic Hydrocarbons in Ungaged Tributaries .................................................................................

Comparison of Loads during Storms and Low Flow.........................................................79

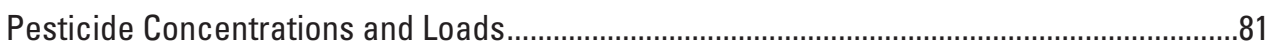

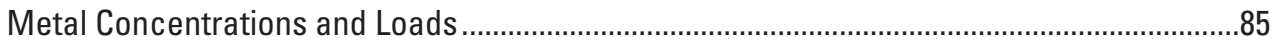

Comparison of Loads of Contaminants of Concern with Total Maximum Daily

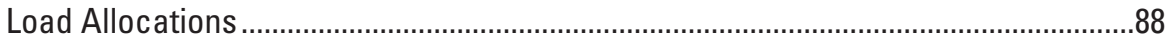

Summary

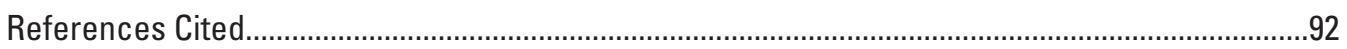


Appendix 1. Summary of stream discharge, precipitation, and sediment and contaminant loadings for the individual storms sampled in tributaries to the Anacostia

River, 2017.

Appendix 2. Summary of polychlorinated biphenyl, polycyclic aromatic hydrocarbon, pesticide, and metal concentrations in blank samples and suspended and bed sediment in tributaries to the Anacostia River, 2017

Appendix 3. Datasets used to model suspended sediment in tributaries to the Anacostia River, 2017.

\section{Figures}

1. Maps showing the Anacostia River watershed sampling locations and subwatersheds: $A$, Anacostia River, $B$, Beaverdam Creek, $C$, Watts Branch, $D$, Hickey Run, E, Nash Run, F, Pope Branch, G, unnamed stream at Fort DuPont, and $H$, unnamed stream at Fort Stanton.

2. Graphs showing stage, turbidity, and precipitation in $A$, Northeast Branch, $B$, Northwest Branch, $C$, Beaverdam Creek, D, Watts Branch, and $E$, Hickey Run during a storm from March 30 through April 2, 2017.

3. Diagram showing configuration of sampling equipment used to collect large-volume and discrete samples.

4. Graph showing stage and water velocity in Beaverdam Creek, March 15-April 1, 2017...

5. Graph showing water levels in Nash Run and Watts Branch, July 18-19, 2017

6. A, Screen capture showing cross-sectional water velocity, and $B$, graph showing turbidity and specific conductance measured March 30, 2017, at Beaverdam Creek.

7. Graphs showing relation between the measured suspended-sediment concentrations in the model dataset and predicted concentrations in $A$, Northeast Branch, $B$, Northwest Branch, $C$, Beaverdam Creek, $D$, Watts Branch, and $E$, Hickey Run

8. Hydrographs showing discharge and measured and predicted suspended-sediment concentrations in $A$, Northeast Branch, $B$, Northwest Branch, $C$, Beaverdam Creek, $D$, Watts Branch, and $E$, Hickey Run for the storm beginning March 31, 2017.

9. Histograms of molar percentage of polychlorinated biphenyls (PCBs) for PCB congeners in samples of suspended sediment during storm and low-flow conditions and samples of bed sediment from $A$, Northeast Branch, $B$, Northwest Branch, $C$, Beaverdam Creek, $D$, Watts Branch, $E$, Hickey Run, $F$, Nash Run, $G$, Pope Branch, $H$, unnamed stream at Fort DuPont, and $I$, unnamed stream at Fort Stanton. 


\section{Tables}

1. U.S. Geological Survey sampling locations

2. Summary of precipitation in the Washington, D.C., area, 2013-17

3. Analytical methods used to measure concentrations of polychlorinated biphenyls, polycyclic aromatic hydrocarbons, organochlorine pesticides, and metals in suspended and bed sediment from tributaries to the Anacostia River, Washington, D.C., 2017.

4. Chemical analytes measured in suspended and bed sediment in tributaries to the Anacostia River, Washington, D.C., 2017.

5. Toxic equivalency factors for polychlorinated biphenyl and polycyclic aromatic

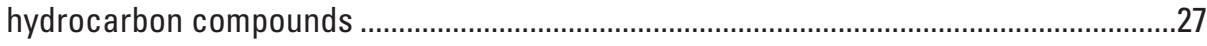

6. Sample identifiers and associated field or equipment blanks .....................................28

7. Concentrations of polychlorinated biphenyls in suspended-sediment samples from tributaries to the Anacostia River.

8. Concentrations of polychlorinated biphenyl compounds in samples of bed sediment from tributaries to the Anacostia River.

9. Concentrations of polycyclic aromatic hydrocarbon compounds in suspendedsediment samples from tributaries to the Anacostia River....

10. Concentrations of polycyclic aromatic hydrocarbon compounds in bed sediment in tributaries to the Anacostia River

11. Concentrations of selected pesticides in suspended-sediment samples from tributaries to the Anacostia River.

12. Concentrations of selected pesticides in bed sediment in tributaries to the Anacostia River

13. Concentrations of metals in suspended-sediment samples from tributaries to the Anacostia River

14. Concentrations of metals in bed sediment in tributaries to the Anacostia River.

15. Estimated sediment loads for Northeast Branch, Northwest Branch, Watts Branch, and Hickey Run, 2017

16. Discharge and stream measurements for Nash Run, Pope Branch, Fort DuPont Creek, and Fort Stanton Creek.......................................................................................4

17. Relative basin areas and estimated discharge for ungaged tributaries, 2013-17...........45

18. Summary of available discharge, turbidity, and suspended-sediment concentration data for the Anacostia River tributaries.

19. Summary of stream data used for regression analysis of Anacostia River tributaries.

20. Regression models for predicting suspended-sediment concentrations in the Anacostia River tributaries

21. Summary of yearly discharge and turbidity in the continuous records for Anacostia River tributaries compared with model dataset.

22. Annual discharge and predicted sediment load for tributaries to the Anacostia River.

23. Estimated sediment loads in ungaged tributaries to the Anacostia River, calendar year 2017

24. Summary statistics of polychlorinated biphenyl and polycyclic aromatic hydrocarbon concentrations in tributaries to the Anacostia River 
25. Loads and relative contribution of total polychlorinated biphenyls and polycyclic aromatic hydrocarbons in tributaries to the Anacostia River in 2017.

26. Summary of sediment and chemical concentrations and loads in tributaries for storm event on March 31, 2017, compared with low-flow loads... . .81

27. Method detection levels for pesticides in suspended-sediment and bed-sediment samples collected from Beaverdam Creek.

28. Maximum loads of pesticides in tributaries to the Anacostia River, 2017.......................83

29. Total maximum loads of pesticides from all gaged tributaries to the Anacostia River, 2017

30. Average concentrations of selected metals in suspended sediment from tributaries to the Anacostia River, 2017.

31. Loads of suspended-sediment-bound metals in tributaries to the Anacostia River, 2017 .86

32. Average concentrations of selected constituents in tributaries to the Anacostia River grouped into categories

33. Loadings of selected constituents in tributaries to the Anacostia River compared with permitted total maximum daily load allocations. 


\section{Conversion Factors}

U.S. customary units to International System of Units

\begin{tabular}{|c|c|c|}
\hline Multiply & By & To obtain \\
\hline \multicolumn{3}{|c|}{ Length } \\
\hline inch (in.) & 0.0254 & meter $(\mathrm{m})$ \\
\hline foot $(\mathrm{ft})$ & 0.3048 & meter $(\mathrm{m})$ \\
\hline mile (mi) & 1.609 & kilometer (km) \\
\hline \multicolumn{3}{|c|}{ Area } \\
\hline acre & 4,047 & square meter $\left(\mathrm{m}^{2}\right)$ \\
\hline acre & 0.4047 & hectare (ha) \\
\hline acre & 0.4047 & square hectometer $\left(\mathrm{hm}^{2}\right)$ \\
\hline acre & 0.004047 & square kilometer $\left(\mathrm{km}^{2}\right)$ \\
\hline square foot $\left(\mathrm{ft}^{2}\right)$ & 929.0 & square centimeter $\left(\mathrm{cm}^{2}\right)$ \\
\hline square foot $\left(\mathrm{ft}^{2}\right)$ & 0.09290 & square meter $\left(\mathrm{m}^{2}\right)$ \\
\hline square inch $\left(\mathrm{in}^{2}\right)$ & 6.452 & square centimeter $\left(\mathrm{cm}^{2}\right)$ \\
\hline \multicolumn{3}{|c|}{ Volume } \\
\hline gallon (gal) & 3.785 & liter (L) \\
\hline gallon (gal) & 0.003785 & cubic meter $\left(\mathrm{m}^{3}\right)$ \\
\hline gallon (gal) & 3.785 & cubic decimeter $\left(\mathrm{dm}^{3}\right)$ \\
\hline million gallons (Mgal) & 3,785 & cubic meter $\left(\mathrm{m}^{3}\right)$ \\
\hline cubic inch $\left(\mathrm{in}^{3}\right)$ & 16.39 & cubic centimeter $\left(\mathrm{cm}^{3}\right)$ \\
\hline cubic yard $\left(\mathrm{yd}^{3}\right)$ & 0.7646 & cubic meter $\left(\mathrm{m}^{3}\right)$ \\
\hline \multicolumn{3}{|c|}{ Flow rate } \\
\hline foot per second ( $\mathrm{ft} / \mathrm{s})$ & 0.3048 & meter per second $(\mathrm{m} / \mathrm{s})$ \\
\hline foot per minute (ft/min) & 0.3048 & meter per minute $(\mathrm{m} / \mathrm{min})$ \\
\hline cubic foot per second $\left(\mathrm{ft}^{3} / \mathrm{s}\right)$ & 0.02832 & cubic meter per second $\left(\mathrm{m}^{3} / \mathrm{s}\right)$ \\
\hline cubic foot per day $\left(\mathrm{ft}^{3} / \mathrm{d}\right)$ & 0.02832 & cubic meter per day $\left(\mathrm{m}^{3} / \mathrm{d}\right)$ \\
\hline gallon per minute (gal/min) & 0.06309 & liter per second (L/s) \\
\hline gallon per day (gal/d) & 0.003785 & cubic meter per day $\left(\mathrm{m}^{3} / \mathrm{d}\right)$ \\
\hline million gallons per day (Mgal/d) & 0.04381 & cubic meter per second $\left(\mathrm{m}^{3} / \mathrm{s}\right)$ \\
\hline $\begin{array}{l}\text { million gallons per day per square } \\
\text { mile }\left[(\mathrm{Mgal} / \mathrm{d}) / \mathrm{mi}^{2}\right]\end{array}$ & 1,461 & $\begin{array}{l}\text { cubic meter per day per square } \\
\text { kilometer }\left[\left(\mathrm{m}^{3} / \mathrm{d}\right) / \mathrm{km}^{2}\right]\end{array}$ \\
\hline \multicolumn{3}{|c|}{ Mass } \\
\hline ounce, avoirdupois (oz) & 28.35 & $\operatorname{gram}(\mathrm{g})$ \\
\hline pound, avoirdupois (lb) & 0.4536 & kilogram (kg) \\
\hline
\end{tabular}

Temperature in degrees Celsius $\left({ }^{\circ} \mathrm{C}\right)$ may be converted to degrees Fahrenheit ( $\left.{ }^{\circ} \mathrm{F}\right)$ as follows:

$$
{ }^{\circ} \mathrm{F}=\left(1.8 \times{ }^{\circ} \mathrm{C}\right)+32 .
$$

Temperature in degrees Fahrenheit $\left({ }^{\circ} \mathrm{F}\right)$ may be converted to degrees Celsius $\left({ }^{\circ} \mathrm{C}\right)$ as follows:

$$
{ }^{\circ} \mathrm{C}=\left({ }^{\circ} \mathrm{F}-32\right) / 1.8 \text {. }
$$




\section{Datum}

Vertical coordinate information is referenced to the North American Vertical Datum of 1988 (NAVD 88).

Horizontal coordinate information is referenced to the North American Datum of 1983 (NAD 83).

Altitude, as used in this report, refers to distance above the vertical datum.

\section{Supplemental Information}

Specific conductance is given in microsiemens per centimeter at 25 degrees Celsius $\left(\mu \mathrm{S} / \mathrm{cm}\right.$ at $\left.25^{\circ} \mathrm{C}\right)$.

\section{Abbreviations}

$\begin{array}{ll}\text { ADCP } & \text { acoustic Doppler current profiler } \\ \text { ADVM } & \text { acoustic Doppler velocity meter } \\ \text { BCF } & \text { bias correction factor } \\ \text { CAS } & \text { Chemical Abstract Service } \\ \text { COC } & \text { contaminant of concern } \\ \text { DOEE } & \text { Washington, D.C., Department of Energy \& Environment } \\ \text { EPA } & \text { U.S. Environmental Protection Agency } \\ \text { FDP } & \text { Fort DuPont Creek } \\ \text { FNU } & \text { Formazin Nephelometric Units } \\ \text { FSt } & \text { Fort Stanton Creek } \\ \text { HR } & \text { Hickey Run } \\ \text { HRGC/HRMS } & \text { high-resolution gas chromatography/high-resolution mass spectrometry } \\ \text { HMW } & \text { high molecular weight } \\ \text { IUAP } & \text { International Union of Pure and Applied Chemistry } \\ \text { LBDC } & \text { Beaverdam Creek } \\ \text { LF } & \text { low flow } \\ \text { LMW } & \text { low molecular weight } \\ \text { LV } & \text { large-volume sample } \\ \text { MSPE } & \text { model standard percentage error } \\ \text { MDL } & \text { method detection level } \\ \text { MLR } & \text { multivariable linear regression } \\ \text { NR } & \text { Nash Run }\end{array}$




\begin{tabular}{ll} 
NEB & Northeast Branch Anacostia River \\
NPDES & National Pollutant Discharge Elimination System \\
NWB & Northwest Branch Anacostia River \\
PAH & polycyclic aromatic hydrocarbon \\
PB & Pope Branch \\
PCB & polychlorinated biphenyl \\
PTFE & polytetrafluoroethylene \\
POC & particulate organic carbon \\
RMSE & root mean squared error \\
SLR & simple linear regression \\
SS & suspended sediment \\
SSC & suspended-sediment concentration \\
TEF & toxic equivalency factor \\
TEO & toxic equivalency \\
TMDL & total maximum daily load \\
USGS & U.S. Geological Survey \\
WB & Watts Branch \\
WHO & World Health Organization \\
$<$ & greater than \\
\hline &
\end{tabular}




\title{
Sediment and Chemical Contaminant Loads in Tributaries to the Anacostia River, Washington, District of Columbia, 2016-17
}

\author{
By Timothy P. Wilson
}

\section{Abstract}

A study was conducted by the U.S. Geological Survey (USGS) in cooperation with the Washington, D.C., Department of Energy \& Environment to estimate the loads of suspended-sediment-bound chemical compounds in five gaged tributaries and four ungaged tributaries of the Anacostia River (known locally as "Lower Anacostia River") in Washington, D.C. Tributaries whose discharge is measured by the USGS are the Northeast and Northwest Branches of the Anacostia River, referred to in this report as "Northeast Branch" (NEB) and "Northwest Branch" (NWB), respectively; Watts Branch (WB); and Hickey Run (HR). A USGS streamflow-gaging station was established in 2016 on Beaverdam Creek (known locally as "Lower Beaverdam Creek" [LBDC]) to support this study. The ungaged streams studied include Nash Run; Pope Branch; an unnamed stream at Fort DuPont, referred to in this report as "Fort DuPont Creek"; and an unnamed stream at Fort Stanton, referred to in this report as "Fort Stanton Creek." The gaged streams were sampled during four to five storms and two low-flow events during January, March, May, and July 2017. The ungaged streams were sampled during one storm and one low-flow event during July 2017. Storm sampling involved collecting large-volume (60- to 70-liter) composite samples, then removing sediment by filtration in the laboratory. Low-flow samples were obtained by filtering streamwater directly in the field. Continuously recording data sondes were deployed throughout the study to measure turbidity and other water-quality characteristics. During sampling, multiple discrete samples of streamwater were collected to determine suspended-sediment concentration (SSC) and particulate organic carbon (POC) concentration. Shortly after each storm, bed sediment was collected for chemical analysis.

Sediment samples were analyzed for 209 polychlorinated biphenyl (PCB) congeners; 35 polyaromatic hydrocarbon (PAH) compounds, including 20 nonalkylated and 15 alkylated species; and 20 organochlorine pesticide (OP) compounds. Sediment from one storm was analyzed for 23 metals.
Relations were developed among turbidity, discharge, and measured SSC by using multiple linear regression of $\log$-transformed data. These relations were used to estimate SSC from continuous records of discharge and turbidity and were subsequently used to estimate sediment loads for the 2017 calendar year. USGS continuous records of turbidity in NEB, NWB, Watts Branch, and Hickey Run were available for 2013-17, which allowed sediment loads to be calculated for these years. Sediment loads for the ungaged streams were estimated by using loads measured in Watts Branch adjusted on the basis of stream-basin areas.

Sediment loads for 2017 total $3.10 \times 10^{7}$ kilograms $(\mathrm{kg})$, with $1.02 \times 10^{7} \mathrm{~kg}$ (33 percent of total) from the NEB, $1.55 \times 10^{7} \mathrm{~kg}(50$ percent $)$ from the NWB, $4.45 \times 10^{6} \mathrm{~kg}$ (14 percent) from LBDC, $5.62 \times 10^{5} \mathrm{~kg}$ ( 2 percent) from Watts Branch, and $2.82 \times 10^{5} \mathrm{~kg}$ (1 percent) from Hickey Run. Sediment yields were highest from NWB and LBDC $\left(3.13 \times 10^{5}\right.$ kilograms per year per square mile $\left[\mathrm{kg} / \mathrm{yr} / \mathrm{mi}^{2}\right]$ and $3.01 \mathrm{~kg} / \mathrm{yr} / \mathrm{mi}^{2}$, respectively). As a result of gaps in turbidity and discharge data, the load for LBDC reported here was calculated from measurements representing only 88 percent of the year (2017), and thus underestimates the actual load. All other gaged tributaries had datasets covering 100 percent of the year and are considered to fully represent actual loads. Estimated sediment loads for the ungaged streams during 2017 total $3.5 \times 10^{5} \mathrm{~kg}$, with $1.2 \times 10^{5} \mathrm{~kg}$ from Nash Run, $6.2 \times 10^{4} \mathrm{~kg}$ from Pope Branch, $1.1 \times 10^{5} \mathrm{~kg}$ from Fort DuPont Creek, and $5.6 \times 10^{4} \mathrm{~kg}$ from Fort Stanton Creek.

Concentrations of PCBs, PAHs, and chlorinated pesticides in streamwater are presented for stormflow and low-flow conditions. Average concentrations (in stormflow and lowflow samples) of total PCBs (sum of all congeners, including coelutions) are 5.9 micrograms per kilogram $(\mu \mathrm{g} / \mathrm{kg})$ for NEB, $6.6 \mu \mathrm{g} / \mathrm{kg}$ for NWB, $130 \mu \mathrm{g} / \mathrm{kg}$ for LBDC, $34 \mu \mathrm{g} / \mathrm{kg}$ for Watts Branch, and $69 \mu \mathrm{g} / \mathrm{kg}$ for Hickey Run. Average concentrations of total PAHs (tPAH) (total of nonalkylated and alkylated species) are 2,000 $\mu \mathrm{g} / \mathrm{kg}$ for NEB, 3,300 $\mu \mathrm{g} / \mathrm{kg}$ for NWB, $2,200 \mu \mathrm{g} / \mathrm{kg}$ for LBDC, $2,400 \mu \mathrm{g} / \mathrm{kg}$ for Watts Branch, and $18,000 \mu \mathrm{g} / \mathrm{kg}$ for Hickey Run. tPAH concentrations among 
the ungaged streams were highest in Nash Run $(5,500 \mu \mathrm{g} / \mathrm{kg})$; concentrations in the other ungaged streams were less than $(<)$ $700 \mu \mathrm{g} / \mathrm{kg}$.

The general magnitude of $\mathrm{PCB}$ and tPAH concentrations in streamwater samples was low-flow samples greater than $(>)$ stormflow samples greater than or equal to $(\geq)$ bed-sediment samples. PCB congener profiles in the three types of samples were nearly identical in each stream and were similar in all streams except for LBDC, where the dominant PCBs shifted to the lighter di- through tetra- homologs. LBDC showed higher tPCB concentrations and a distinct congener profile from the other streams. The similarity in congener makeup supported that averaging PCB concentrations in stormflow and low-flow samples was appropriate for calculating chemical loads.

Loads of tPCB, tPAH (total of alkylated and nonalkylated forms), and pesticides were estimated for each stream by multiplying average contaminant concentrations by the respective sediment loads. Total PCB loads for 2017 were estimated to be 820 grams (g) with 8 percent $(60 \mathrm{~g})$ from NEB, 12 percent $(95 \mathrm{~g})$ from NWB, 75 percent $(590 \mathrm{~g})$ from LBDC, 3 percent $(25 \mathrm{~g})$ from Watts Branch, and 2.5 percent $(19 \mathrm{~g})$ from Hickey Run. PCB toxicity totaled $3.8 \times 10^{-3} \mu \mathrm{g} / \mathrm{kg}$, with the largest contribution (47 percent) derived from LBDC. Total PAH loads (sum of alkylated and nonalkylated forms) for 2017 were estimated to be $89,000 \mathrm{~g}$, with 23 percent $(20,000 \mathrm{~g})$ from NEB, 59 percent $(52,000 \mathrm{~g})$ from NWB, 11 percent $(9,800 \mathrm{~g})$ from LBDC, 2 percent $(1,400 \mathrm{~g})$ from Watts Branch, and 6 percent $(5,200 \mathrm{~g})$ from Hickey Run. These results indicate that the largest contributor (75 percent) of PCBs to the Anacostia River is LBDC, although it contributes only 15 percent of the sediment and its basin area represents only 10 percent of the area of the Anacostia River watershed. The majority of the PAH load originates from NWB (59 percent of total) and NEB (22 percent). The ungaged tributaries contribute extremely small loads of PCBs and PAHs, totaling $8.1 \mathrm{~g}$ and $765 \mathrm{~kg}$, respectively. More than 94 percent of the total load from the ungaged tributaries is derived from the Nash Run Basin.

Various organochlorine pesticides were present in suspended and bed sediment from all gaged and ungaged tributaries; however, elevated detection levels associated with the analytical methods resulted in numerous unquantifiable concentrations in the suspended-sediment samples. Only the pesticide chlordane was found in measurable concentrations in all gaged tributaries. As a result, in this report, a combination of analytical data from suspended-sediment and bed-sediment samples was used to estimate the maximum pesticide loading for each tributary. Chlordane was the principal compound present in the gaged tributaries; the highest average concentration (average of stormflow and low-flow samples from each stream) was $62 \mu \mathrm{g} / \mathrm{kg}$ in sediment from Watts Branch. Chlordane loads for 2017 totaled $1,100 \mathrm{~g}$, of which 7 percent (430 g) was from NEB, 28 percent (320 g) was from NWB, 28 percent $(310 \mathrm{~g})$ was from LBDC, 5 percent $(56 \mathrm{~g})$ was from Watts Branch, and 1 percent $(11 \mathrm{~g})$ was from Hickey Run. Chlordane was not present in suspended or bed sediment from any of the ungaged tributaries. Loads of the other pesticides were estimated by using the highest concentration measured in the combined suspended-sediment and bed-sediment data for each stream. Notable loads include dieldrin ( $860 \mathrm{~g}$ from NWB), methoxychlor (205 g from LBDC), endrin aldehyde (150 $\mathrm{g}$ from NWB), and 4,4-DDT (79 $\mathrm{g}$ from Watts Branch). Compared with pesticide loads from the gaged streams, those from the ungaged streams were minimal, with only the Pope Branch contribution exceeding 1 gram per year for 4,4-DDE $(1.05 \mathrm{~g})$ and $4,4^{\prime}$-DDT $(1.3 \mathrm{~g})$.

The results of this study show that the dominant source of PCBs and chlordane is LBDC, despite its relatively small basin area. PAHs are ubiquitous throughout the study area, with the largest sources being NEB and NWB; this finding is a result of the large sediment load originating from these basins. The small, ungaged streams supply only minimal PCB and PAH loads, with Nash Run being the largest contributor.

\section{Introduction}

The Anacostia River is a major tributary to the Potomac River, one of the several large rivers flowing into Chesapeake Bay. Originating in eastern Maryland, the Northeast Branch of the Anacostia River and Northwest Branch of the Anacostia River, referred to in this report as the "Northeast Branch" (NEB) and "Northwest Branch" (NWB), respectively, flow south toward Washington, D.C., where they join to form the lower Anacostia River, which ultimately enters the Potomac River. The Anacostia River flows 8 miles (mi) through the District of Columbia; along this reach, several small tributaries join the Anacostia River. The water, sediment, and contaminants that are delivered to the Anacostia River are the focus of this study.

Over the past decade, the Anacostia River has been the focus of much study as part of an effort to restore the quality of the river (D.C. Department of Energy \& Environment, 2012). The remediation and cleanup efforts began with a "Phase I Remedial Investigation" (RI) study (TetraTech, 2016). This study identified the lack of data on tributary loadings of sediment and chemicals of concern (COCs) as being an important deficiency in the information needed for the remedial effort. Determining whether these tributaries represent ongoing sources of contaminated sediment is critical, not only for source track-down and cleanup efforts but also to provide input for a hydrodynamic and water-quality model being developed for the Anacostia River.

In response to this need, the U.S. Geological Survey (USGS), in cooperation with the Washington, D.C., Department of Energy \& Environment (DOEE), initiated a study to determine the current-day (2017) loadings of sediment and COCs from the five larger, gaged tributaries and four smaller, ungaged tributaries to the Anacostia River. The larger tributaries are the NEB and NWB of the Anacostia River, Beaverdam Creek (known locally as "Lower Beaverdam Creek" [LBDC]), 
Watts Branch (WB), and Hickey Run (HR). The four smaller ungaged tributaries are Nash Run (NR); Pope Branch (PB); an unnamed stream at Fort DuPont, referred to in this report as "Fort DuPont Creek" (FDP); and an unnamed stream at Fort Stanton, referred to in this report as "Fort Stanton Creek"(FSt). To determine the present-day (2017) loadings of sediment, samples were collected during storms and during low-flow conditions. The suspended materials were separated and analyzed for sediment-bound COCs that included polychlorinated biphenyls (PCBs), polycyclic aromatic hydrocarbons (PAHs), organochlorine pesticides, and, to a lesser degree, trace metals. The data obtained and loads calculated in this study begin to fill the existing data gaps for the remediation efforts and provide the information needed by the DOEE to track the sources of contaminants and sediment.

Remediation seeks to identify areas of contamination, then to quantify the magnitudes of the various sources of contaminants to those areas, thereby providing a basis for prioritizing cleanup and source-removal efforts. Many remediation studies have demonstrated that tributaries commonly are the largest sources of contaminated sediment to the mainstem of rivers. The tributary basins of the Anacostia River vary greatly in size and discharge, but all share two common attributes of being situated in a highly urbanized area and being the receiving water bodies for extensive stormwater-collection networks.

USGS discharge records show the NEB and NWB are the two largest basins in the Anacostia River watershed and supply most of the water to the river (Miller and others, 2007, 2013). Discharge of two mid-sized tributaries, Watts Branch and Hickey Run, has been measured by the USGS since 2013. Prior to the present study, little information was available on the discharge from the LBDC and from the several small, ungaged urban streams that enter the Anacostia River. The relative magnitudes of discharge of the other ungaged tributaries is unknown at present (2017); however, all the small streams act as conduits for water collected by the storm-sewer systems that exist throughout the Washington, D.C., metropolitan area. Storm drains efficiently capture and deliver water, sediment, and chemicals washed off roadways and paved lots, discharged from gutters on commercial and residential buildings, and moving overland as runoff from lots and yards. The runoff from impervious surfaces is the principal pathway by which chemicals associated with wet and dry precipitation are transferred to urban waterways. Storm drains are also susceptible to being used for illegal waste disposal.

The goal of this study was to estimate current (2017) loads of COCs to the Anacostia River. Three data types are needed to estimate tributary loadings. First, an accurate, continuous record of the volume of water moving through each tributary is needed. Second, because of the hydrophobic nature of COCs, an accurate accounting of the suspended material delivered by the flowing water is needed. And third, representative concentrations of the COCs associated with suspended materials during stormflow (when most sediment is transported) and low-flow conditions (when the finest grain-sized materials are transported) are needed.
This study was undertaken to provide as accurate an accounting as possible of discharge, sediment loads, and representative concentrations and loads of COCs in the tributaries for 2017. This task was accomplished by combining continuously measured discharge with suspended-sediment concentrations (SSCs) estimated from continuously measured turbidity. The resulting sediment loadings were multiplied by the concentrations of chemicals associated with the suspended sediment (SS). To this end, SS was collected throughout the year during four to six storm events (depending on the stream); one to two samples were also collected under low-flow conditions. Large-volume (LV) composite samples were collected to obtain sufficient mass of sediment for high-resolution analysis. Several aspects of this work deserve comment.

1. This study was conducted under the constraint that sampling was to be completed during 2017. As with all storm-sampling projects, weather conditions affect the outcome of the study. It was not possible to archive storm samples in the hope that a "more appropriate" storm would be forthcoming. Unless severe equipment failure occurred, once collected, the sediment was sent for analysis regardless of the magnitude of the storm or the coverage of the sampling over the storm. Although rare, equipment failures or other unavoidable conditions resulted in short gaps in the stream-turbidity data and the collection of samples.

2. The concentrations reported for the individual storms and low-flow conditions are considered to be "typical" of those occurring over the remainder of the year. The relatively few samples obtained preclude statistical testing to demonstrate similarities and differences. Because of the paucity of chemical data, yearly loads were calculated by using average concentrations in storm and low-flow samples.

3. Chemical concentrations during storm events were calculated by using estimates of dry-sediment weight captured on filters. Tests showed that the percent moisture in wet-sediment-laden filters averaged 70 percent +17 percent. This is one source of uncertainty associated with the chemical concentrations presented in this report. Although a propagation of error calculation was not performed, the analytical uncertainty resulting from moisture content is considered to be small compared with the uncertainty in measurements of discharge and in the estimates of SSCs.

4. Where continuous turbidity data are available, sediment loadings for 2013-15 were calculated to demonstrate the relation between sediment loads and yearly precipitation. Pre-2017 sediment loadings may be used to estimate COC loadings, but concentrations of COCs in previous years likely differed from those measured in this study. 
5. One purpose of sampling the small, ungaged streams was to establish the concentrations of COCs in these streams, thereby indicating the need for determining contaminant sources. The continuous discharge and sediment loadings in these streams were not measured directly but were estimated from data measured for WB. A correction was not applied for impervious/pervioussurface areas in the small basins, nor for other factors that affect runoff; rather, all precipitation falling in the basins of the ungaged tributaries was assumed to enter the tributary. Loadings, therefore, are considered to be maximum values. The small basin sizes of the ungaged tributaries dictate that discharge and sediment loadings are small compared to those of the larger, gaged streams, as demonstrated by the results of this study. It is also likely that the loads estimated for the ungaged basins are within the range of uncertainty associated with the loadings from even the smallest of the gaged tributaries.

6. Discrete SS and particulate organic carbon (POC) samples were collected during individual storm events only to help establish the relation between turbidity and SSCs needed to predict continuous sediment concentrations. The SSC data for any storm will not cover the entire range of concentrations that actually occurred in the stream, as indicated from the continuous turbidity record. Therefore, using the average SSC for samples during an event will not produce a concentration (and therefore a load) that fully represents the range of sediment concentrations that occurred during the storm.

7. Sampling on Hickey Run was conducted immediately downstream from a sediment- and trash-collection structure located at the New York Avenue bridge. Prior to the summer of 2017, this structure was observed to be overflowing with sediment and trash. The trap was cleaned in the summer of 2017. The effect of the structure on the loading of sediment and the contribution of chemicals leached from entrapped trash is unknown, but it is reasonable to assume that the trap affected the sediment chemistry in the stream, and consequently the loadings calculated for the stream. If maintenance of the trap continues, future loadings will likely differ from those presented herein.

8. Pesticide compounds, especially chlordane, are of special concern to the Anacostia River remediation program (Phelps, 2005). As discussed farther on in this report, the method detection levels (MDLs) for suspended sediment were unexpectedly higher than the MDLs for bed sediment. As a result, only a few compounds were quantifiable in the suspended-sediment samples. As a compromise, the combined datasets of suspended and bed sediment were used to establish loadings. Loadings should, therefore, be considered to be maximums, and a "nondetect" reported for a pesticide is not evidence of its absence in the stream. Moreover, because collection of samples for pesticide analysis began in the summer of 2017, fewer measurements are available, and these may not capture the actual range in concentrations present in the streams. Importantly, only chlordane was found in quantifiable concentrations in the suspended sediment from all gaged tributaries - these concentrations were used exclusively to estimate loads.

\section{Purpose and Scope}

This report describes the methods used to sample suspended sediment in gaged and ungaged tributaries of the Anacostia River during storm and low-flow conditions during 2016 and 2017, and presents results of chemical analyses for SSC, POC, PCBs, PAHs, pesticides, and trace metals in the suspended sediment and bed sediment. Relations are developed among turbidity, SSC, and discharge and are used with continuously measured turbidity to calculate sediment loadings. These sediment loadings are then combined with the chemical concentrations to estimate loadings of COCs in the Anacostia River.

\section{Study Area}

Many reports associated with the Anacostia River remediation program are available and describe in detail the geologic, geomorphologic, topographic, and cultural aspects of the tributary basins in this study (Warner and others, 1996; Miller and others, 2007, 2013; TetraTech, 2016). Therefore, a detailed review of the basin is not presented here, and the reader is directed to the various studies referenced in the Phase I Remedial Investigation (TetraTech, 2016). The brief descriptions of the small tributary basins below were developed from the final total maximum daily loads (TMDLs) for organic compounds and metals in the Anacostia River and its tributaries (D.C. Department of Health, 2003). These descriptions focus on the physical characteristics that affect the hydrologic responses of the streams.

\section{Description of Watersheds}

Maps of the Anacostia River watershed and its individual subwatersheds are presented in figure 1; the locations of sampling points in each watershed are listed in table 1. Figure 1 shows the outlines of theAnacostia watershed and each subwatershed boundary, the stormwater sewersheds within the Washington, D.C., city boundary (D.C. Department of Health, 2003), the locations where sampling was conducted, and the portion of each basin contributing water and sediment to the sampling locations. Three characteristics can be gleaned from 
these maps. First, the maps show that the streams are crossed by many roadways and the basins are highly urbanized with few open-land areas; typically, these are riparian corridors through which the streams flow. Impervious-surface coverages as high as 80 percent are reported for these basins (D.C. Department of Health, 2003). Second, the many roadways have an associated network of stormwater sewers throughout the basins. The stormwater sewersheds coincide directly with, but in some cases extend beyond the boundary of, the topographic basins. As a result of the extensive coverage of impervious surfaces and the sewer systems, nearly all the precipitation that falls on these basins likely reaches the stream shortly after the onset of precipitation. A "flashy" hydrologic response of the streams results as precipitation is quickly routed from roadways, sidewalks, roofs, and parking areas into the streams. Third, the land surface along the southeastern bank of the Anacostia River slopes steeply; this gradient induces high water-flow velocities in the streams, on the paved surfaces, and through the collection system. High velocities can cause extensive erosion of the land surface and streambanks. Eroded material may contain contaminants from past land uses, and is undoubtedly augmented by grit, sediment, and contaminants washed off paved and unpaved surfaces. The Washington, D.C., government has conducted extensive work to identify and reduce streambank erosion in these areas in an effort to reduce future loadings.

The response of water flow and sediment in a stream to precipitation reflects the physical makeup of the basin and the extent of stormwater collection in the basin. To illustrate the similarities and differences among the tributaries, hydrographs of stage and turbidity are presented for the storm sampled on March 31, 2017 (fig. 2). This storm was associated with a regional weather front passing through the Washington, D.C., area that delivered precipitation to all the tributary basins. A total of 0.965 inch (in.) of rain was recorded at the Ronald Reagan Washington National Airport (an intensity of 0.064 inch per hour [in/hr]) on March 31. The interval when precipitation occurred is indicated on each hydrograph.

The subwatersheds differ greatly in drainage area size (fig. 1A, table 1). The basins of NEB and NWB are the largest watersheds studied. NEB has a total channel length (sum of the major and all minor tributaries) of $12 \mathrm{mi}$; the channel length of NWB is considerably longer $(75 \mathrm{mi})$. These basins cover areas of 72.5 square miles $\left(\mathrm{mi}^{2}\right)$ and $49.4 \mathrm{mi}^{2}$, respectively. As expected, these two tributaries supply most of the tributary input $(32,900$ million gallons [Mgal]) to the Anacostia River; USGS streamflow data for 2017 indicate that NEB provided 48 percent of the combined flow, with NWB providing 33 percent of the total flow. These tributaries join to form the Anacostia River just upstream from the Washington, D.C., boundary (fig. 1). The two tributaries flow from the Piedmont to the Coastal Plain physiographic province (Miller and others, 2007), and both basins have similar land coverages. Impervious-surface area ranges from about 20 percent in the upper portions of the basins to nearly 50 percent in the areas near the confluence (Washington, D.C., Department of Energy \& Environment, 2012). Both basins are densely populated, with 25.1 and $21.9 \mathrm{mi}^{2}$ of land classified as residential in the NEB and NWB, respectively; 2.3 and $10.3 \mathrm{mi}^{2}$ are classified as forested and 3.8 and $7.7 \mathrm{mi}^{2}$ are classified as agricultural, respectively. Two National Pollutant Discharge Elimination System (NPDES) discharge sites - municipal wastewater-treatment plants operated by the U.S. Department of Agriculture - are present in the NEB and NWB Basins. Light-industrial-waste sites, potential sources of metals derived from paint-pigment manufacturing and metal electroplating, also are present (Miller and others, 2007, 2013). Sources of petroleum hydrocarbons include parking areas associated with commercial facilities, and mixed wastes derived from military facilities in the NEB and NWB basins (Miller and others, 2007, 2013).

The hydrographs for the NEB and NWB for the March 31 storm event (figs. $2 A$ and $2 B$ ) are broad with rounded peaks and slowly receding limbs - typical of hydrographs for large streams. However, stream stage in both basins rose rapidly, within 1 hour of the onset of precipitation. Both streams typically displayed at least two peaks in stage; the first is indicative of the arrival of water from storm drains in the basins, and the second is associated with the rise in stage as water reaches the gage from the upland area of the basin. Stage then receded slowly, taking 2 to 3 days before reaching prestorm levels. In both basins, water turbidity generally mimicked stage throughout the storm. Turbidity in the NEB showed a sharp spike lasting 30 minutes at the initial rise in stage/discharge; during the remainder of the storm, the turbidity mimicked the stage but was extremely "noisy," especially late in the hydrograph. This behavior may be the result of turbulence in the stream channel in the area near the gaging station that keeps fine-grained sediment circulating in suspension. In the NWB, turbidity also mimicked stage but shows well-defined peaks associated with "slugs" of materials moving downstream. In the NEB, the turbidity trace shows several large spikes that typically represent only a few turbidity measurements (turbidity was measured at 5-minute intervals).

Beaverdam Creek (fig. $1 B$ ) is a mid-sized basin covering $14.9 \mathrm{mi}^{2}$. LBDC enters Washington, D.C., from Maryland near the I-495/Route 50 interchange, then flows southwest along Route 50 through the northeastern corner of Washington, D.C., before entering the Anacostia River. Over its 27-mi channel length, LBDC is fed by several small tributaries, including Cabin Brook. The complete boundary of the LBDC sewershed is not shown in figure $1 B$ because it incorporates a portion of the watershed outside the D.C. city boundary. However, many storm drains - for example, five large stormwater drains in the concrete structure under the Route 50 bridge-were observed along the banks, indicating substantial stormwater input to the stream. It is likely that stormwater is also routed into LBDC upstream from the D.C. boundary.

The impervious cover in the LBDC is reported to be 32 percent, with residential land use characterizing roughly half the basin area $\left(6.8 \mathrm{mi}^{2}\right)$; the remainder is commercial 
and industrial $\left(0.97 \mathrm{mi}^{2}\right)$, forested $\left(3.6 \mathrm{mi}^{2}\right)$, and agricultural $\left(6.6 \mathrm{mi}^{2}\right)$ (TetraTech, 2016). The forested areas include the riparian corridor, a large cemetery, and a golf course. The LBDC Basin is crossed by several large roads (Route 295, Route 50) and two railroad corridors that dissect the residential and commercial areas. Over much of its length, LBDC runs through a wooded riparian corridor immediately adjacent to Route 50 and a railroad right-of-way. One of the most prominent land uses in the basin is an extensive recycling plant facility located just upstream from the sampling location.

Because discharge information was not available for LBDC, a gaging station was installed about $0.3 \mathrm{mi}$ downstream from the Route 50 bridge. The stream at this location is affected by the tidal fluctuations in the Anacostia and Potomac Rivers. During 2017, discharge data showed that LBDC supplied approximately 16 percent of the total tributary water input to the Anacostia River.

The hydrograph for LBDC (fig. 2C) shows the effect of the tides, with a daily fluctuation of 2.0 to 2.5 feet (ft). During low-flow (prestorm) conditions, stage was typically less than $0.5 \mathrm{ft}$ and water velocity was near 0 feet per second $(\mathrm{ft} / \mathrm{s})$. Water movement also decreased to near $0 \mathrm{ft} / \mathrm{s}$ as maximum stage was approached. Little or no upstream flow of water was measured; it is likely that water cannot pass upstream from the Route 50 bridge. Stage typically responded within 20 to 30 minutes from the onset of precipitation and rose rapidly, overprinting the normal tidal fluctuation. Turbidity began to increase within a few minutes of the start of the rise in stage and lagged slightly behind stage. After the stage and turbidity curves peak, both smoothly recede until the onset of the next rise in tide. Over the next 2 to 3 days, the low water stage between each tide cycle continues to decrease but remains above the prestorm level. The number of tide cycles before stage returns to prestorm levels is related to the magnitude of the storm event and conditions in the Anacostia River. Turbidity, however, continues to decline and shows no discernable effect from the subsequent tidal cycles. The turbidity record shows that sediment entrained during the storm continued moving downstream after normal tidal cycling resumed.

Watts Branch Basin (fig. $1 C$ ) is the next tributary basin downstream from the LBDC Basin. The Watts Branch Basin covers an area of $3.36 \mathrm{mi}^{2}$, with 80 percent $\left(2.6 \mathrm{mi}^{2}\right)$ of the land use listed as residential (D.C. Department of Health, 2003; D.C. Department of Environmental Programs, 2014). Less than 15 percent of the basin area is forested $\left(0.46 \mathrm{mi}^{2}\right)$; forested area is mainly along the tree-lined stream corridor. During 2017, Watts Branch contributed 2 percent of the total tributary discharge to the Anacostia River.

Watts Branch originates as two smaller tributaries that begin along the northeast-southwest-trending ridgeline (at an altitude of approximately $175 \mathrm{ft}$ above the North American Vertical Datum of 1988 (NAVD 88)). The western tributary originates at a pond in a golf course located between Marlboro Pike and Brooke Road, near the town of Oakland, Maryland. This limb flows northward until reaching East Capital Street, where it crosses the Washington, D.C., boundary. The eastern tributary rises along the ridgeline near Rollings Avenue and flows northward to its confluence with the western limb. The two limbs join just inside the northeastern corner of the District. The channel then turns northwest and flows under major roadways until it joins the Anacostia River. Watts Branch is approximately $4 \mathrm{mi}$ long and flows entirely above ground, passing through residential areas. The boundary of the sewershed (fig. 1C) is truncated at the Washington, D.C., boundary, but the sewer system in Maryland likely connects to the sewers in the D.C. portion of the basin.

During the March storm, both stage and turbidity in Watts Branch (fig. $2 D$ ) rose quickly before cresting. Watts Branch is not affected by tidal fluctuations at the gaging-station location, and stage returned to its prestorm level after about 24 hours. The storm hydrograph shows several small peaks in stage, but the hydrograph is broader than would be anticipated for a flashy urban stream. Multiple, distinct peaks in turbidity are observed.

The Hickey Run Basin is located on the western side of the Anacostia River, across from the Aquatic Gardens and the mouth of Nash Run (fig. 1D). The Hickey Run Basin is much smaller than the Watts Branch Basin, with an area of only $1.01 \mathrm{mi}^{2}$ (TetraTech, 2016). Roughly half the topographic basin lies upstream from the sampling point. Hickey Run is unique among the tributaries studied because for most of its length, it flows underground through a concrete conduit. The entire basin upstream from the sampling point is urbanized, consisting of residential areas and several areas of large commercial buildings. The path of the underground channel is unknown, but likely crosses under Route $1 \mathrm{~A}$ and a railroad right-of-way. Many stormwater drains undoubtedly connect to the underground channel. Separate sewersheds in the northeastern corner of the basin may or may not be connected to Hickey Run. After passing under Route 50, the stream flows above ground, passing through a sediment weir and trash strainer before continuing through the National Arboretum and then into the Anacostia River. The sediment load in Hickey Run is affected by the sediment-trap structure located just upstream from the sampling station. This structure consists of a concrete weir intended to divert trash and debris into a second structure that acts as a strainer. From late 2016 through early summer 2017, the weir was observed to be filled with sediment, allowing water and sediment to simply pass over the weir. After it was cleaned, the weir operated properly, and the stream flowed through the strainer.

Hickey Run (fig. 2E) responded almost instantaneously with the onset of precipitation. Its hydrograph exhibits multiple separate peaks, including one distinct peak late in the receding limb. Turbidity was chaotic and unlike that observed in the other tributaries. Multiple peaks in turbidity occurred just after the onset of precipitation; following these peaks, turbidity eventually peaked about 8 hours after precipitation ceased. The early peaks represent "slugs" of sediment that may have been loose material that moved from behind the weir. The slow rise in turbidity likely indicates the arrival of material stored in the underground channel and newer material 
washed off the streets. The slight rise (less than $[<] 0.25 \mathrm{ft}$ ) in stage was accompanied by a well-defined increase in turbidity that occurred approximately 24 hours before the onset of precipitation. Similar peaks are observed throughout the record and are likely caused by releases upstream in the sewershed that subsequently entrained sediment stored behind the weir. Turbidity increased greatly at the very end of the hydrograph; the reason for this observation is unknown, but cloudy water and an oil sheen were commonly observed on the water surface after storm events.

The ungaged streams are located downstream from LBDC along the southeastern side of the Anacostia River; from north to south, they are Nash Run, Pope Branch, Fort DuPont Creek, and Fort Stanton Creek. These streams are in generally elongated basins draining the northeast-southwesttrending ridge that runs along the southeastern edge of Washington, D.C. The ridge is located immediately northwest of Southern Avenue. These small tributaries have steep gradients; they begin at altitudes of 200 to $250 \mathrm{ft}$ above NAVD 88 and flow down to the Anacostia River at an altitude of approximately $5 \mathrm{ft}$ (NAVD 88) over channel lengths of 1 to $2 \mathrm{mi}$. The northeastern side of the ridgeline drains southeast to Oxon Creek and then into the Potomac River.

Nash Run is located directly across the Anacostia River from the mouth of Hickey Run (fig. $1 E$ ) and is between LDBC and Watts Branch. Except where it passes under roadways, Nash Run flows above ground through areas much like the Watts Branch Basin; 95 percent is classified as mixed residential and small industrial/commercial. The watershed covers $0.7 \mathrm{mi}^{2}$, with approximately two-thirds of the watershed located within the D.C. boundary and one-third in Deanwood Park, in Prince Georges County, Maryland. Many stormwater sewers were observed to discharge to Nash Run. As in the Watts Branch Basin, the sewershed boundary is truncated at the D.C. boundary, but the storm sewers likely connect to storm sewers in Maryland.

Pope Branch (fig. $1 F$ ) is in an extremely narrow basin $\left(0.37 \mathrm{mi}^{2}\right)$ located southwest of Fort DuPont Creek. Pope Branch begins along the ridgeline in Fort Davis Park at an altitude of about $250 \mathrm{ft}$ (NAVD 88), then flows through Pope Branch Park for about 1.25 mi to Fairlawn Avenue (altitude approximately $30 \mathrm{ft}$ [NAVD 88]), where it flows into an underground sewer. After entering the conduit, the tributary flows about 1,500 ft until it enters the Anacostia River. The basin is approximately 85 percent residential and light commercial property (U.S. Geological Survey, 2000). The Pope Branch sewershed coincides with the topographic basin, and below its headwaters, the stream receives water from many storm-sewer lines.

The Fort DuPont Creek Basin (fig. $1 G$ ) lies southeast of the Watts Branch Basin and is a rectangular-shaped basin that extends from the southeast-trending ridgeline to Fairlawn Avenue. The stream begins near Alabama Avenue at an altitude of about $250 \mathrm{ft}$ (NAVD 88) and flows down to the railroad right-of-way, where it flows underground (at approximately $30 \mathrm{ft}$ [NAVD 88]) for about 1,100 ft to the Anacostia River.
The Fort DuPont watershed covers an area of about $0.68 \mathrm{mi}^{2}$, of which approximately 90 percent is within Fort DuPont Park (U.S. Army Corps of Engineers, 2009a). The park is a grasscovered, hilly area containing a golf course and few roads. The stormwater sewershed and the topographic basin coincide; the stream receives runoff from impervious areas within the park and in the neighborhoods outside the park.

Fort Stanton Creek (fig. $1 H$ ) is the southernmost of the ungaged tributaries and has a basin area of about $0.33 \mathrm{mi}^{2}$. The creek emerges from a pipe in a corner of Fort Stanton Park, located along the ridgeline at an altitude of about $200 \mathrm{ft}$ (NAVD 88). Once above ground, it flows 2,100 ft until it enters a storm drain near Good Hope Road. Upstream from the sampling point, the stream flows above ground through wooded parkland surrounded by residential areas. Because the sampling point is upstream from the location where the stream enters the buried conduit at Good Hope Road, the samples represent only a small percentage of the basin. Downstream from Good Hope Road, the stream flows 4,400 ft underground until it reaches the Anacostia River. The large sewershed area downstream from the sampling point likely contributes a substantial volume of water to the discharge that ultimately enters the Anacostia River. Therefore, the results for this tributary presented herein represent only a small part of the basin, and do not represent water and sediment entering the underground sewer pipe.

The characteristics of these ungaged tributaries are as follows. All have steep streambed gradients of about 200 feet per mile (ft/mi), which encourages bank erosion. Although wooded riparian corridors are present, many erosional banks are present in the parklands, especially in the Fort Dupont Creek Basin, where an erosional bank is immediately upstream from Minnesota Avenue. The steep gradients and hilly terrain are source areas for much sediment, and the relations among stream length, discharge, and mass of sediment delivered by the ungaged streams likely differ from those for nearby Watts Branch. These tributaries receive water from numerous stormwater collection systems that drain the roadways and impervious areas in the basins. The stormwater sewersheds cover the entirety of each topographic basin, collecting runoff from miles of roadways and parking areas. Outside the parkland, land use in the basins is mainly residential, commercial, and light industrial in areas that contain gutter systems that route water into the stormwater system and then the tributaries. Nearly all the precipitation that falls on these basins can be assumed to enter the stormwater-collection system, the tributaries, and ultimately the Anacostia River. Water in these small tributaries, most notably Fort DuPont and Fort Stanton Creeks, flows underground for considerable distances downstream from the sampling locations. A substantial volume of stormwater along with sediment and road grit is likely added to the discharge, which ultimately reaches the Anacostia River; this water was not characterized in this study.

Although this report focuses on loads in 2017, possible variations in loads in response to precipitation during previous years were considered. During 2017, air temperatures and the 


\section{A. ANACOSTIA RIVER WATERSHED AND SUBWATERSHEDS}

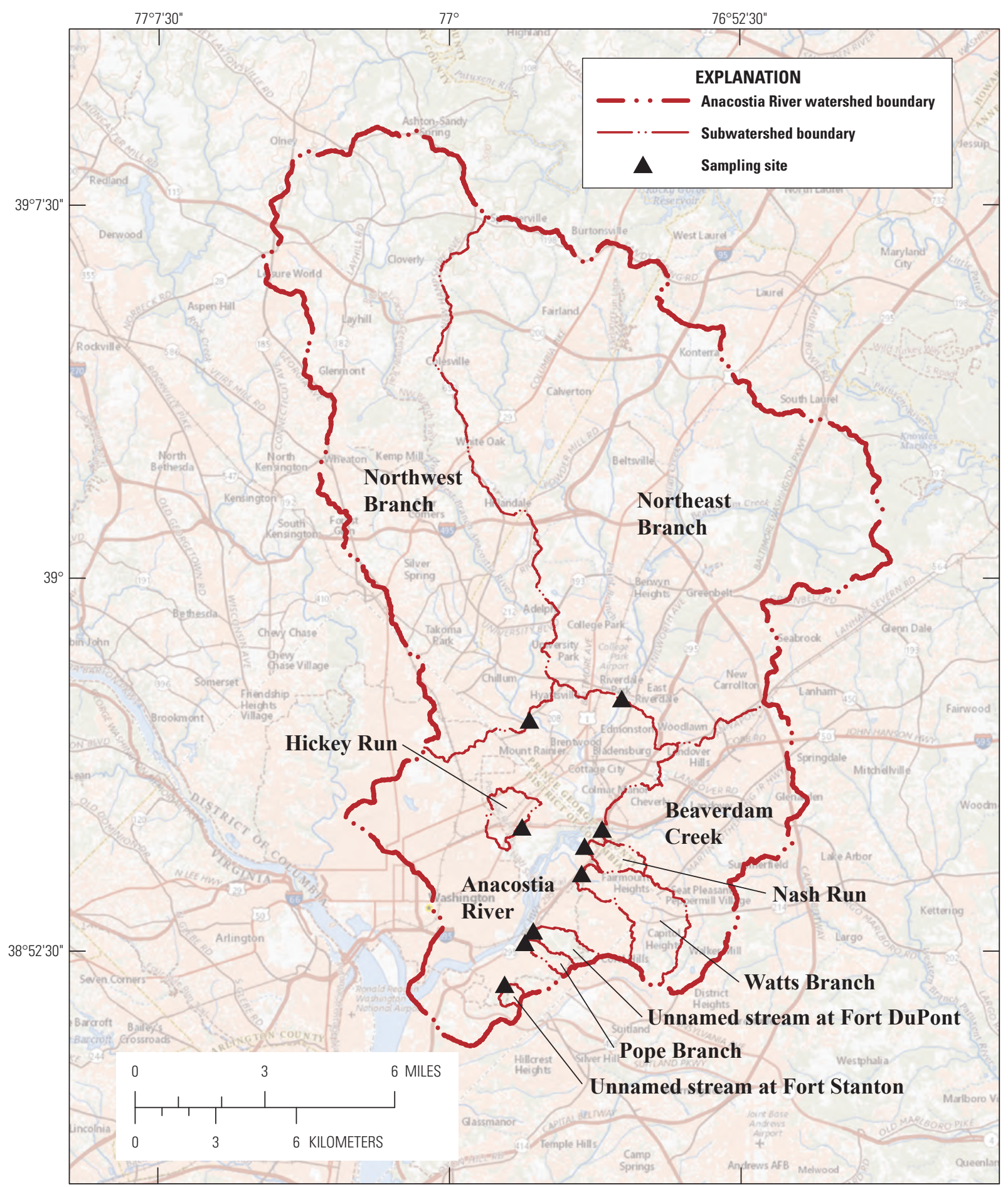

Figure 1. The Anacostia River watershed sampling locations and subwatersheds: $A$, Anacostia River, $B$, Beaverdam Creek, $C$, Watts Branch, D, Hickey Run, E, Nash Run, F, Pope Branch, G, unnamed stream at Fort DuPont (Fort DuPont Creek), and $H$, unnamed stream at Fort Stanton (Fort Stanton Creek). 


\section{B. BEAVERDAM CREEK}

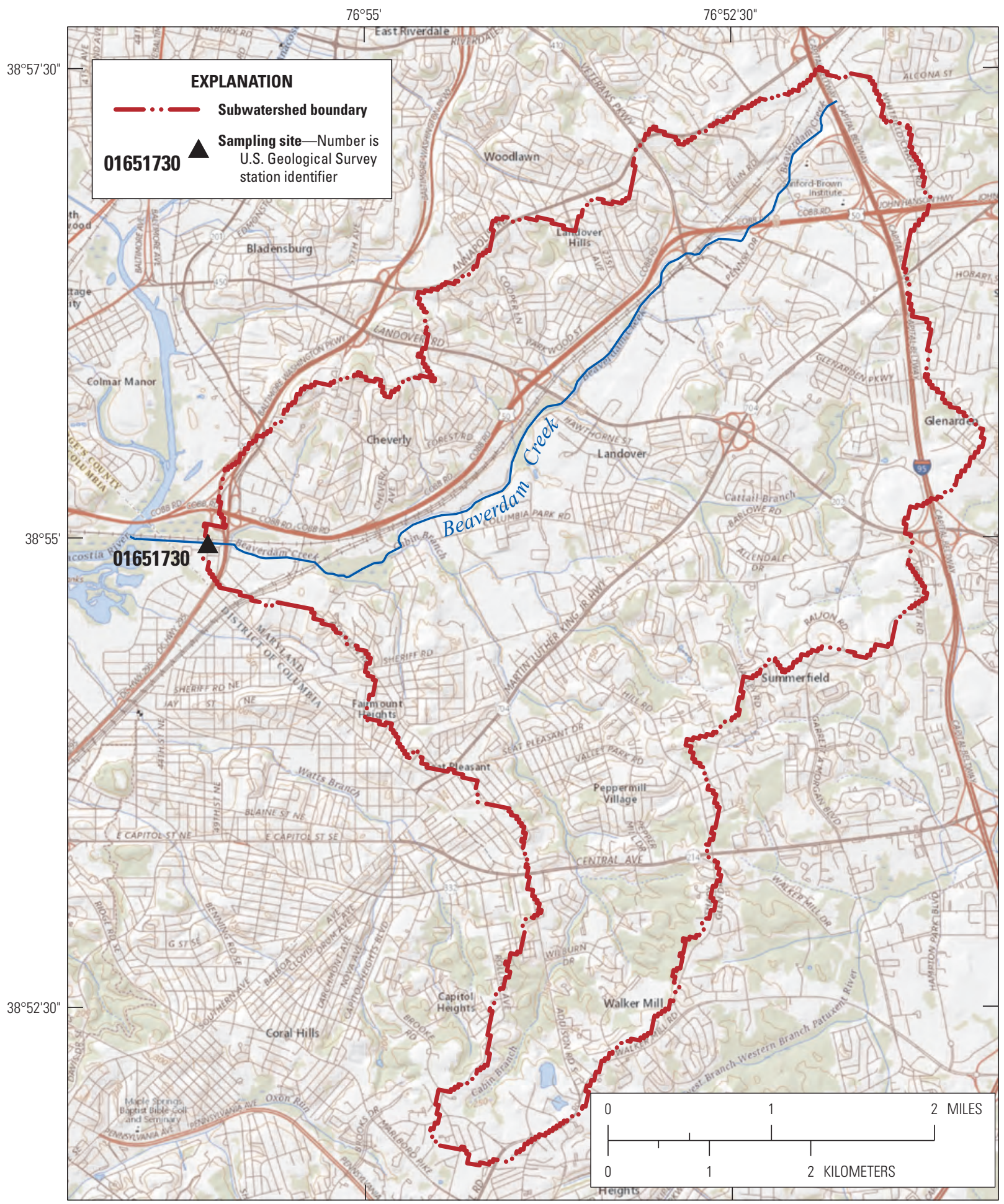

Base from U.S. Geological Survey, The National Map, 2017

Figure 1. The Anacostia River watershed sampling locations and subwatersheds: $A$, Anacostia River, $B$, Beaverdam Creek, C, Watts Branch, D, Hickey Run, E, Nash Run, F, Pope Branch, G, unnamed stream at Fort DuPont (Fort DuPont Creek), and $H$, unnamed stream at Fort Stanton (Fort Stanton Creek).-Continued 


\section{WATTS BRANCH}

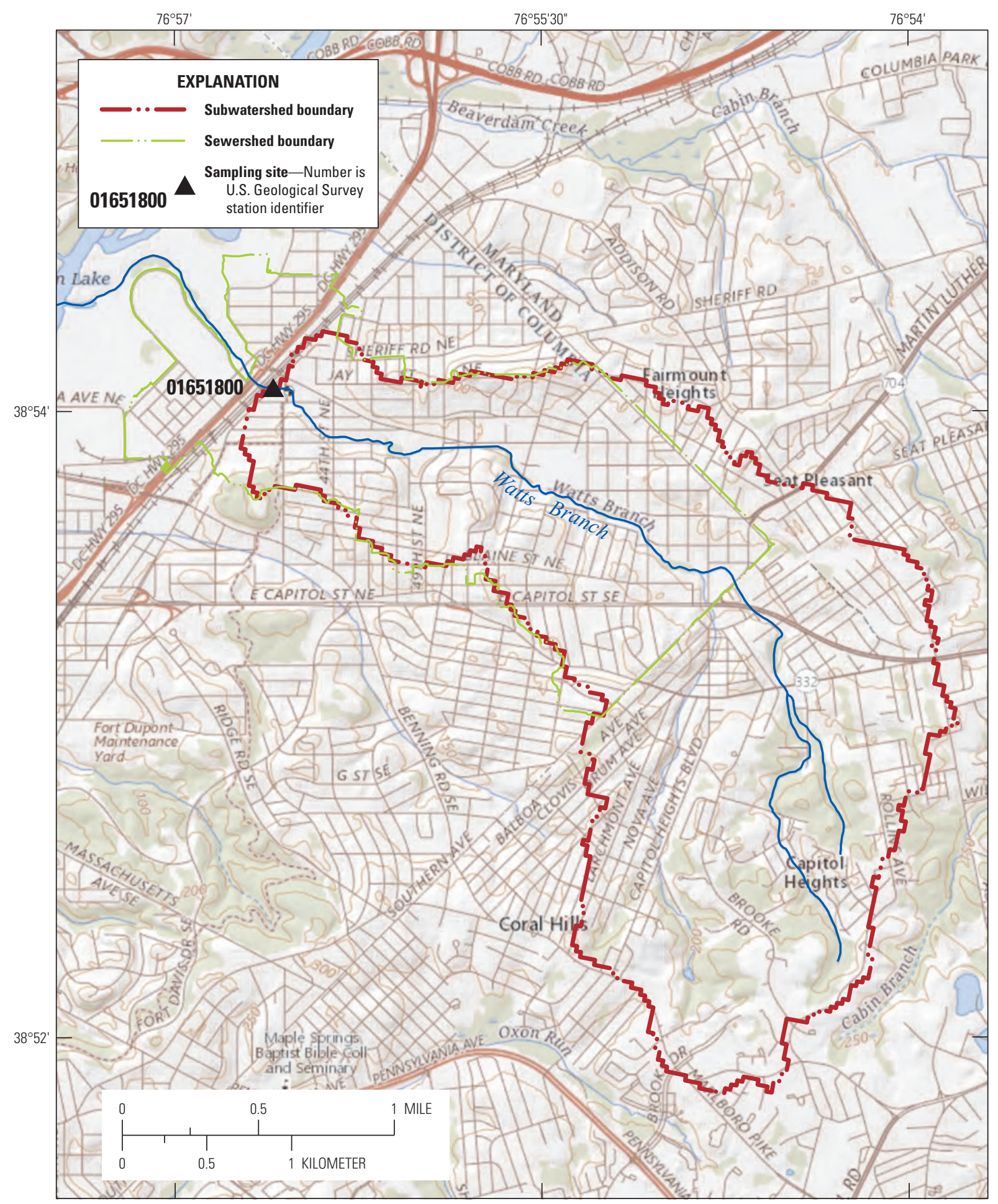

Base from U.S. Geological Survey, The National Map, 2017

Figure 1. The Anacostia River watershed sampling locations and subwatersheds: $A$, Anacostia River, $B$, Beaverdam Creek, C, Watts Branch, D, Hickey Run, E, Nash Run, F, Pope Branch, G, unnamed stream at Fort DuPont (Fort DuPont Creek), and $H$, unnamed stream at Fort Stanton (Fort Stanton Creek).-Continued 


\section{HICKEY RUN}

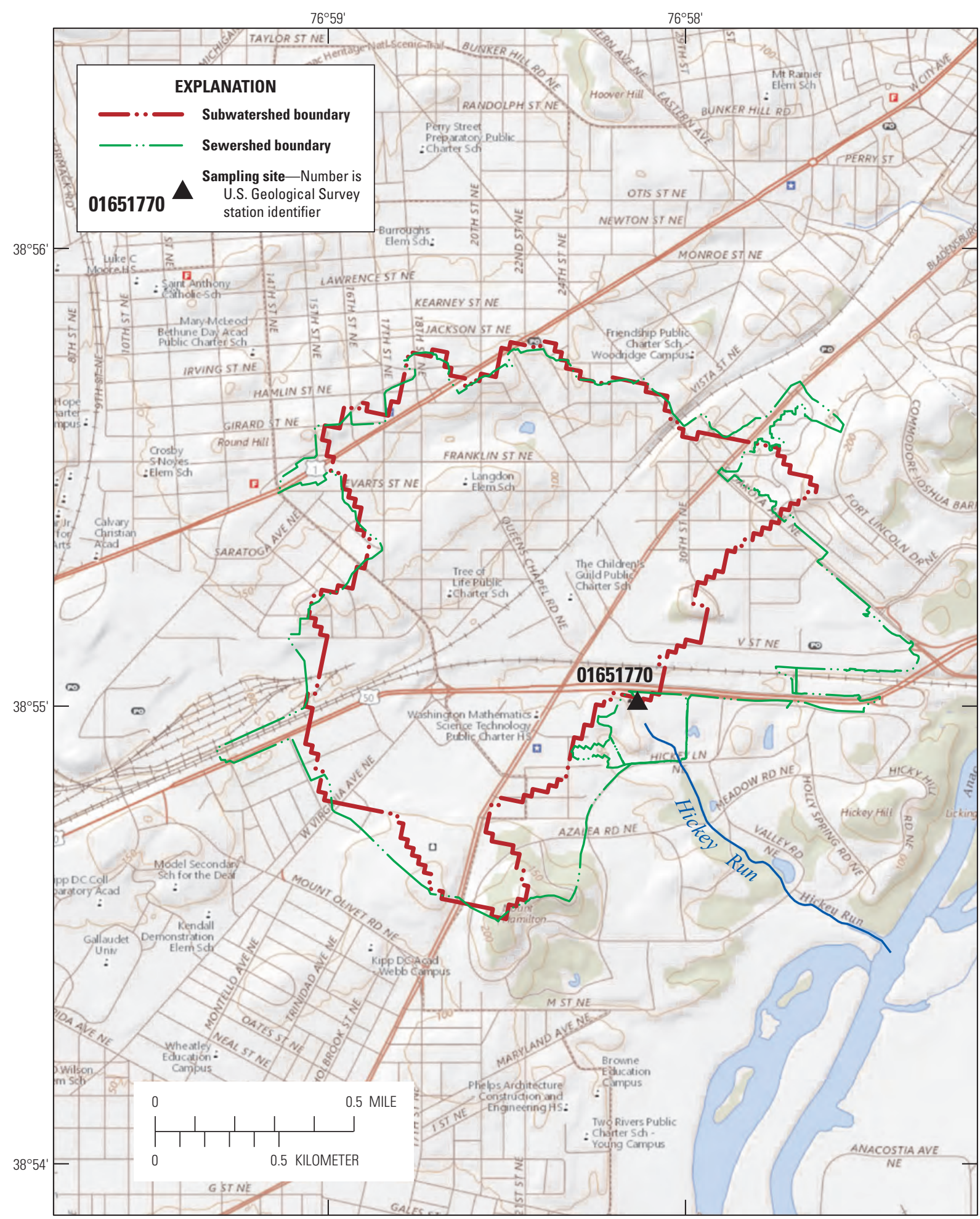

Base from U.S. Geological Survey, The National Map, 2017

Figure 1. The Anacostia River watershed sampling locations and subwatersheds: $A$, Anacostia River, $B$, Beaverdam Creek, C, Watts Branch, D, Hickey Run, E, Nash Run, F, Pope Branch, G, unnamed stream at Fort DuPont (Fort DuPont Creek), and $H$, unnamed stream at Fort Stanton (Fort Stanton Creek).-Continued 


\section{E. NASH RUN}

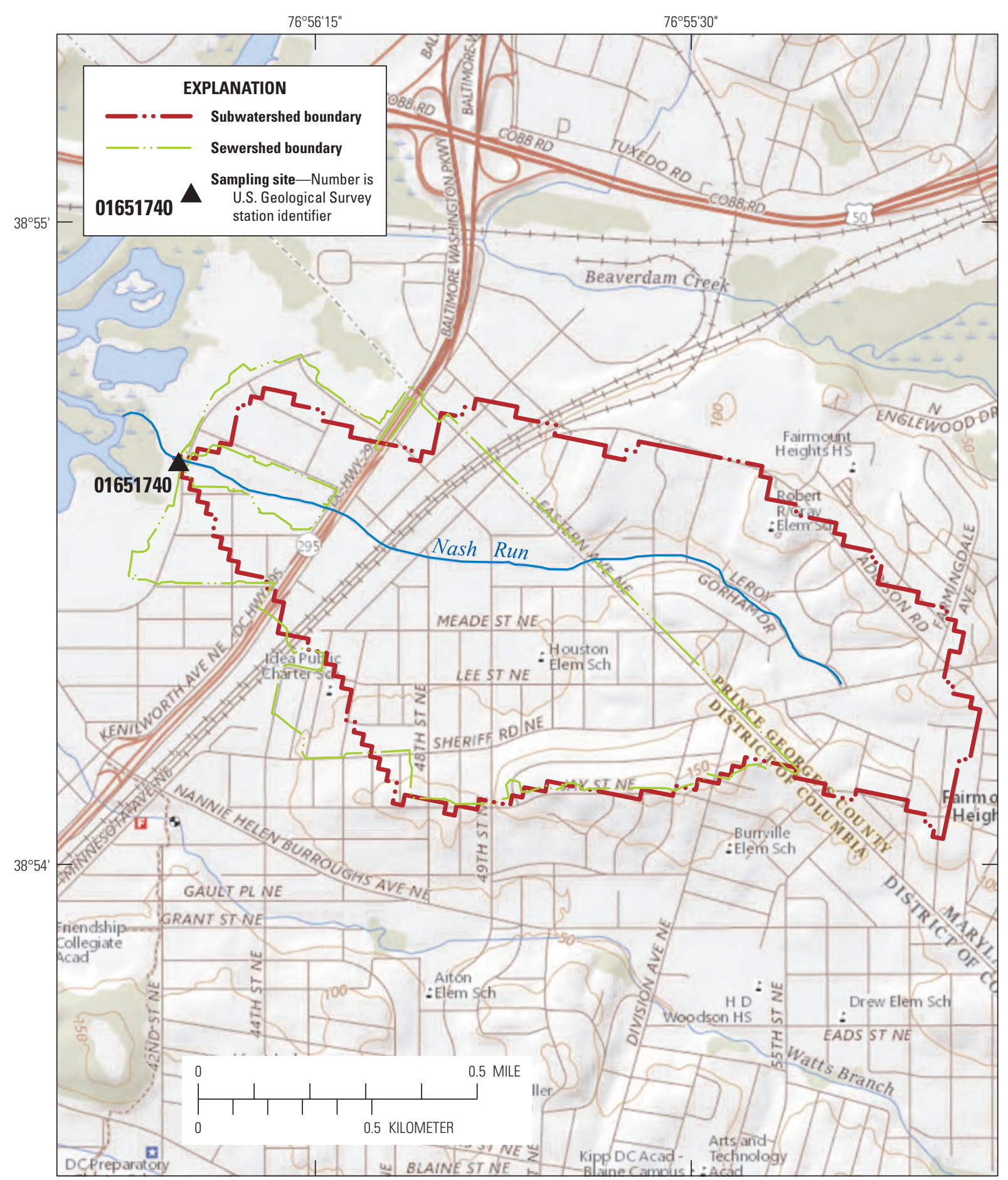

Base from U.S. Geological Survey, The National Map, 2017

Figure 1. The Anacostia River watershed sampling locations and subwatersheds: $A$, Anacostia River, $B$, Beaverdam Creek, $C$, Watts Branch, D, Hickey Run, E, Nash Run, F, Pope Branch, G, unnamed stream at Fort DuPont (Fort DuPont Creek), and $H$, unnamed stream at Fort Stanton (Fort Stanton Creek).-Continued 
F. POPE BRANCH

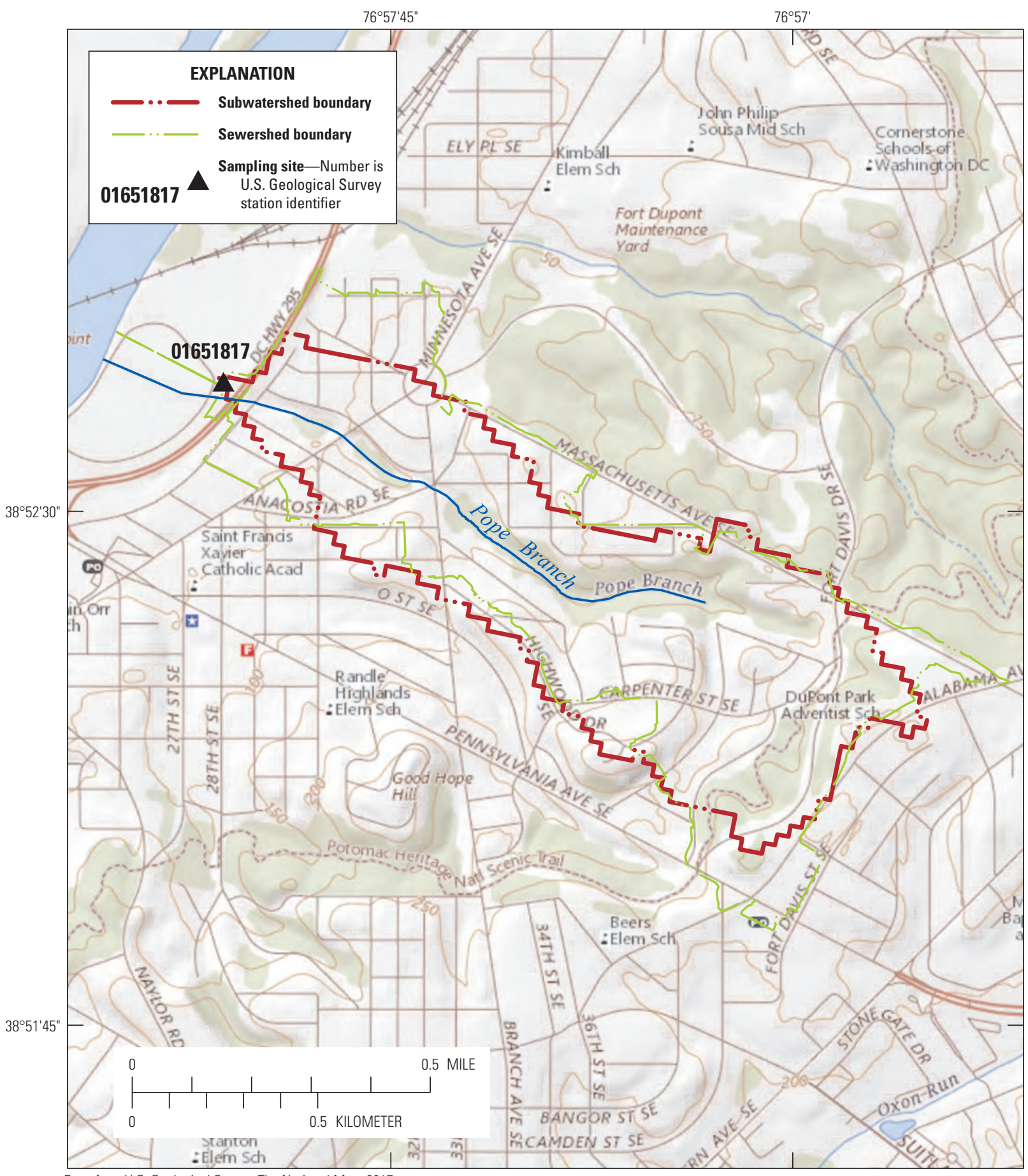

Base from U.S. Geological Survey, The National Map, 2017

Figure 1. The Anacostia River watershed sampling locations and subwatersheds: $A$, Anacostia River, $B$, Beaverdam Creek, C, Watts Branch, D, Hickey Run, E, Nash Run, F, Pope Branch, G, unnamed stream at Fort DuPont (Fort DuPont Creek), and $H$, unnamed stream at Fort Stanton (Fort Stanton Creek).-Continued 
G. UNNAMED STREAM AT FORT DUPONT

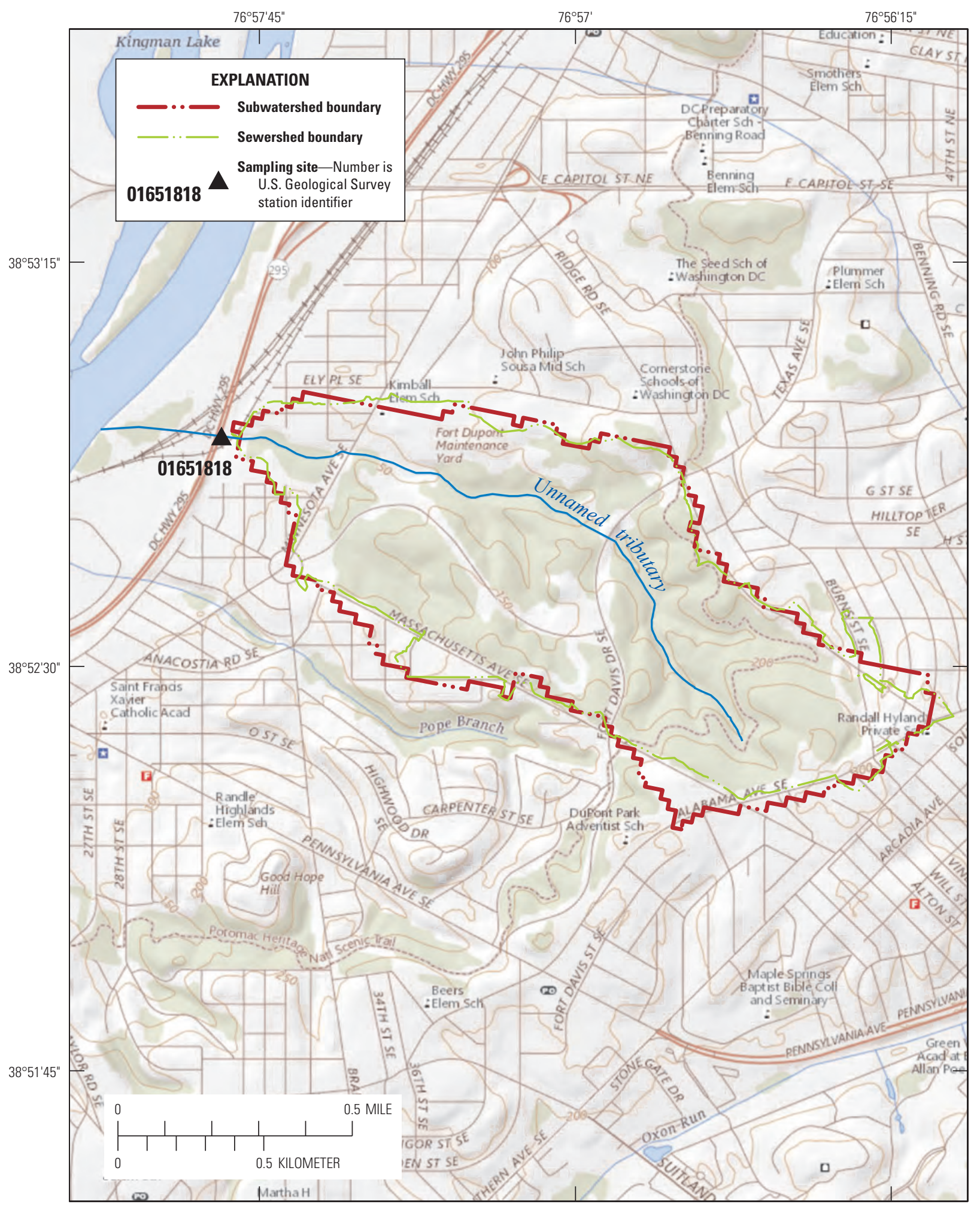

Base from U.S. Geological Survey, The National Map, 2017

Figure 1. The Anacostia River watershed sampling locations and subwatersheds: $A$, Anacostia River, $B$, Beaverdam Creek, C, Watts Branch, D, Hickey Run, E, Nash Run, F, Pope Branch, G, unnamed stream at Fort DuPont (Fort DuPont Creek), and $H$, unnamed stream at Fort Stanton (Fort Stanton Creek).-Continued 


\section{H. UNNAMED STREAM AT FORT STANTON}

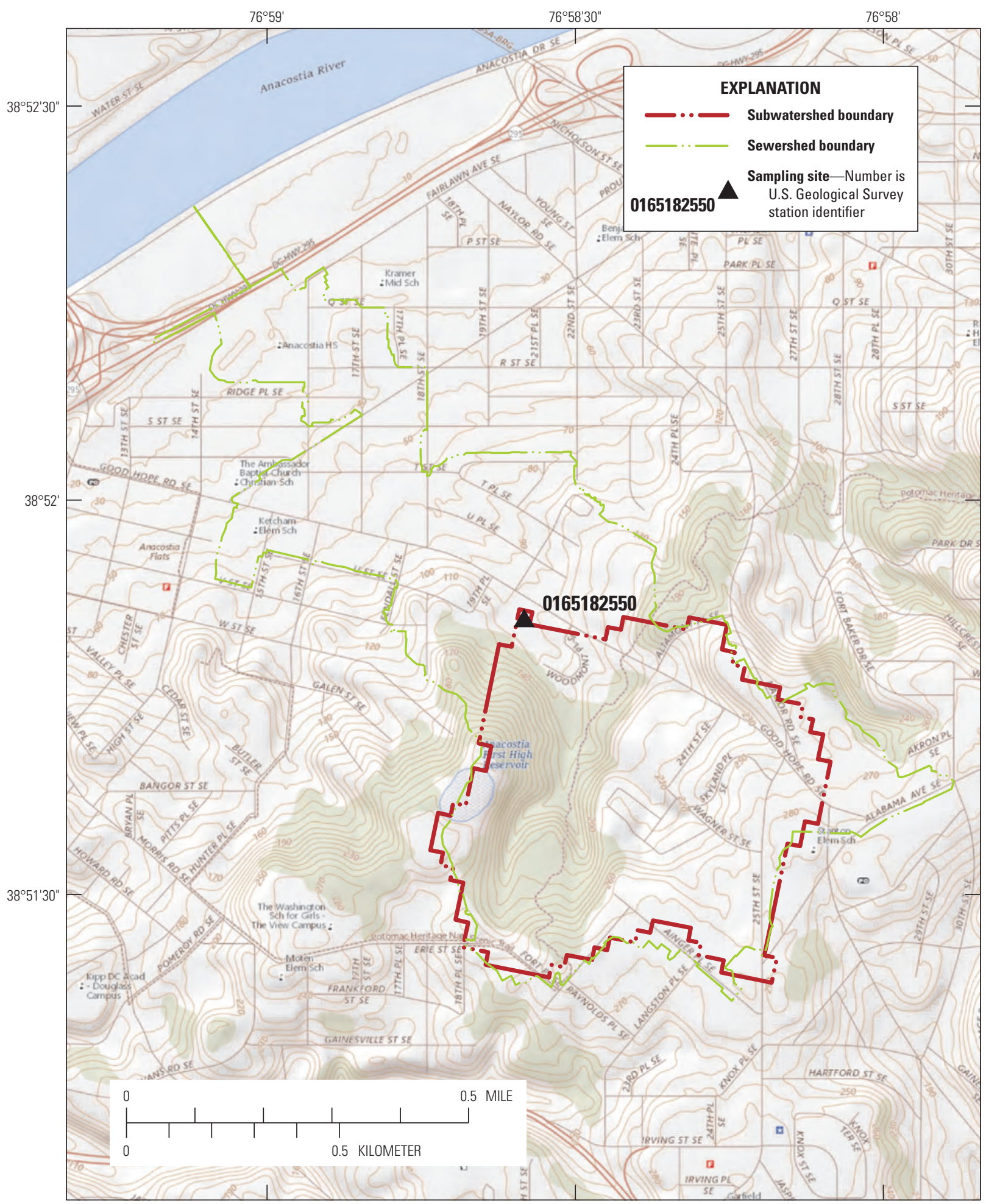

Base from U.S. Geological Survey, The National Map, 2017

Figure 1. The Anacostia River watershed sampling locations and subwatersheds: $A$, Anacostia River, $B$, Beaverdam Creek, C, Watts Branch, D, Hickey Run, E, Nash Run, F, Pope Branch, G, unnamed stream at Fort DuPont (Fort DuPont Creek), and $H$, unnamed stream at Fort Stanton (Fort Stanton Creek).-Continued 
Table 1. U.S. Geological Survey sampling locations.

[NEB, Northeast Branch; NWB, Northwest Branch; LBDC, Beaverdam Creek; WB, Watts Branch; HR, Hickey Run; NR, Nash Run; PB, Pope Branch; FDP, Fort DuPont Creek; FSt, Fort Stanton Creek; mi, miles; ft, feet; latitudes and longitudes are in degrees, minutes, seconds; rte., route]

\begin{tabular}{|c|c|c|c|c|c|}
\hline $\begin{array}{l}\text { U.S. Geological } \\
\text { Survey station } \\
\text { identifier }\end{array}$ & Abbreviation & Name & Location & $\begin{array}{l}\text { Latitude and } \\
\text { longitude }\end{array}$ & $\begin{array}{l}\text { Drainage } \\
\text { area, in } \\
\text { square } \\
\text { miles }\end{array}$ \\
\hline \multicolumn{6}{|c|}{ U.S. Geological Survey gaged stream } \\
\hline 01649500 & NEB & $\begin{array}{l}\text { Northeast Branch Anacostia River at } \\
\text { Riverdale, Md. }\end{array}$ & $\begin{array}{l}\text { Located on left bank } 100 \text { feet downstream from bridge on Riverdale Road, } \\
\text { Riverdale, Md., } 1.8 \text { mi downstream from Indian Creek, and } 1.8 \mathrm{mi} \\
\text { upstream from confluence with Northwest Branch. }\end{array}$ & $\begin{array}{l}38^{\circ} 57^{\prime} 36.9^{\prime \prime} \\
76^{\circ} 55^{\prime} 33.5^{\prime \prime}\end{array}$ & 72.52 \\
\hline 01651000 & NWB & $\begin{array}{l}\text { Northwest Branch Anacostia River near } \\
\text { Hyattsville, Md. }\end{array}$ & $\begin{array}{l}\text { Located on right bank at downstream side of bridge on Queens Chapel } \\
\text { Road (State Highway 500), Hyattsville, Md., } 0.8 \mathrm{mi} \text { downstream from } \\
\text { Sligo Branch, } 1.0 \text { mi west of Hyattsville, and } 1.6 \mathrm{mi} \text { upstream from } \\
\text { confluence with Northeast Branch. }\end{array}$ & $\begin{array}{l}38^{\circ} 57^{\prime} 08.4^{\prime \prime} \\
76^{\circ} 57^{\prime} 57.8^{\prime \prime}\end{array}$ & 49.4 \\
\hline 01651730 & LBDC & Beaverdam Creek near Cheverly, Md. & $\begin{array}{l}\text { Located on left bank, } 500 \mathrm{ft} \text { downstream from highway Rte. } 50 \text { bridge } \\
\text { on Baltimore-Washington Parkway, } 1.0 \text { mi southwest of Cheverly and } \\
0.5 \text { mi upstream from mouth. }\end{array}$ & $\begin{array}{l}38^{\circ} 54^{\prime} 58.7^{\prime \prime} \\
76^{\circ} 56^{\prime} 03.5^{\prime \prime}\end{array}$ & 14.8 \\
\hline 01651800 & WB & Watts Branch at Washington, D.C. & $\begin{array}{l}\text { Located at upstream side of Minnesota Ave. bridge, and } 1.0 \text { mi upstream } \\
\text { from mouth }\end{array}$ & $\begin{array}{l}38^{\circ} 54^{\prime} 04.6^{\prime \prime} \\
76^{\circ} 56^{\prime} 35.8^{\prime \prime}\end{array}$ & 3.36 \\
\hline 01651770 & HR & $\begin{array}{l}\text { Hickey Run at National Arboretum at } \\
\text { Washington, D.C. }\end{array}$ & $\begin{array}{l}\text { Located on left bank } 75 \mathrm{ft} \text { downstream from New York Ave., 1,000 ft west } \\
\text { of entrance to National Arboretum, Washington, D.C. }\end{array}$ & $\begin{array}{l}38^{\circ} 55^{\prime} 00.5^{\prime \prime} \\
76^{\circ} 58^{\prime} 09.2^{\prime \prime}\end{array}$ & 1.01 \\
\hline \multicolumn{6}{|c|}{ Ungaged stream } \\
\hline 01651740 & NR & Nash Run at Washington, D.C. & East side of Anacostia Avenue NE, near Douglas Ave. & $\begin{array}{l}38^{\circ} 54^{\prime} 37.2^{\prime \prime} \\
76^{\circ} 56^{\prime} 31.6^{\prime \prime}\end{array}$ & 0.71 \\
\hline 01651817 & $\mathrm{~PB}$ & $\begin{array}{l}\text { Pope Branch at Fairlawn Avenue at } \\
\text { Washington, D.C. }\end{array}$ & At west end of Fairlawn Avenue, Washington, D.C. & $\begin{array}{l}38^{\circ} 52^{\prime} 39.36^{\prime \prime} \\
76^{\circ} 57^{\prime} 59.83^{\prime \prime}\end{array}$ & 0.34 \\
\hline 01651810 & $\mathrm{FDP}^{1}$ & $\begin{array}{l}\text { Unnamed stream to Anacostia River below } \\
\text { Ft. DuPont at Washington, D.C. }\end{array}$ & East side of railroad crossing and end of 32nd St, SE, Washington, D.C. & $\begin{array}{l}38^{\circ} 52^{\prime} 55.6^{\prime \prime} \\
76^{\circ} 57^{\prime} 50.4^{\prime \prime}\end{array}$ & 0.68 \\
\hline 0165182550 & $\mathrm{FSt}^{1}$ & $\begin{array}{l}\text { Unnamed stream to Anacostia River above } \\
\text { Good Hope Road at Washington, D.C. }\end{array}$ & $\begin{array}{l}\text { At east end of condominium, } 500 \text { feet south of Good Hope Road } \\
\text { intersection at 19th St. }\end{array}$ & $\begin{array}{l}38^{\circ} 51^{\prime} 50.10^{\prime \prime} \\
76^{\circ} 58^{\prime} 35.70^{\prime \prime}\end{array}$ & 0.22 \\
\hline
\end{tabular}

'In this report, and in all Washington, D.C., Department of Energy \& Environment documentation, these sites are referred to as Fort DuPont Creek and Fort Stanton Creek, respectively. 


\section{A. Northeast Branch}
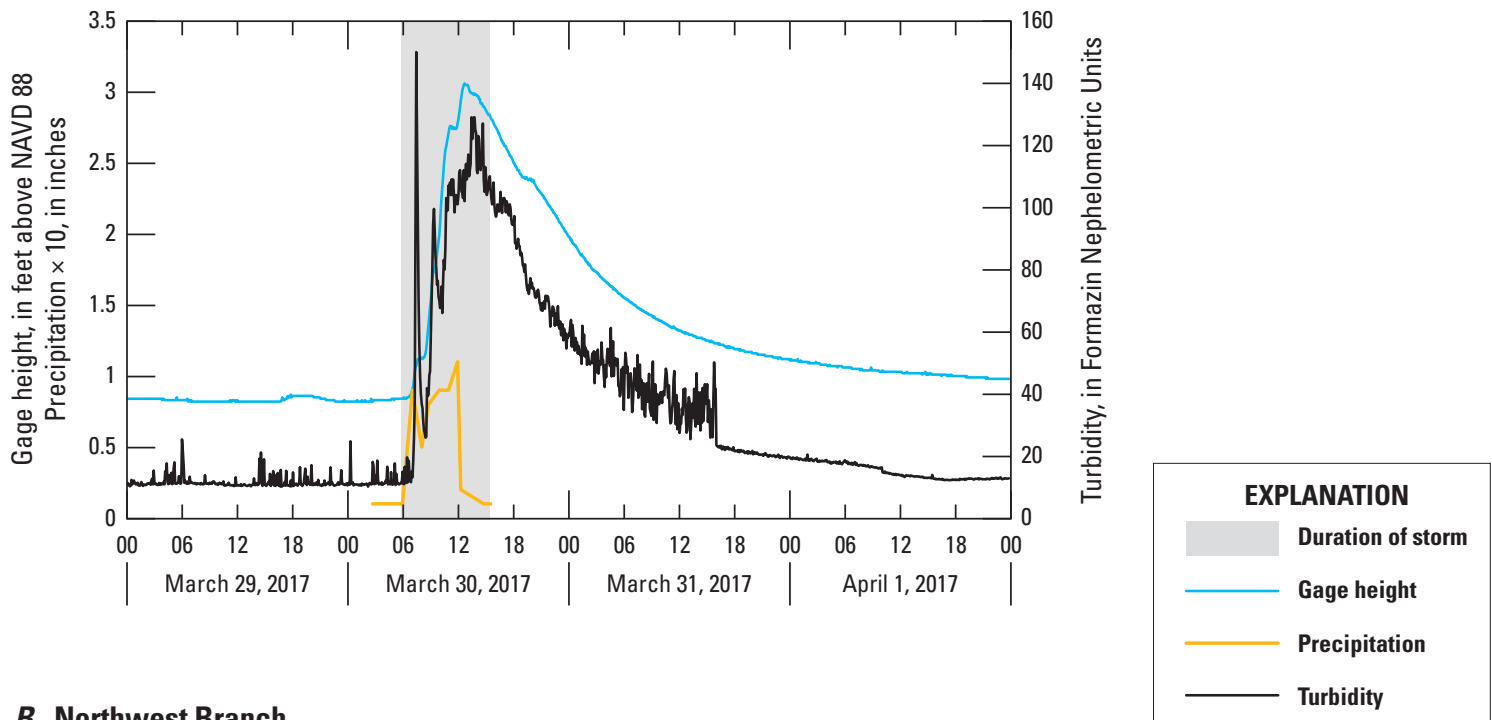

\section{B. Northwest Branch}

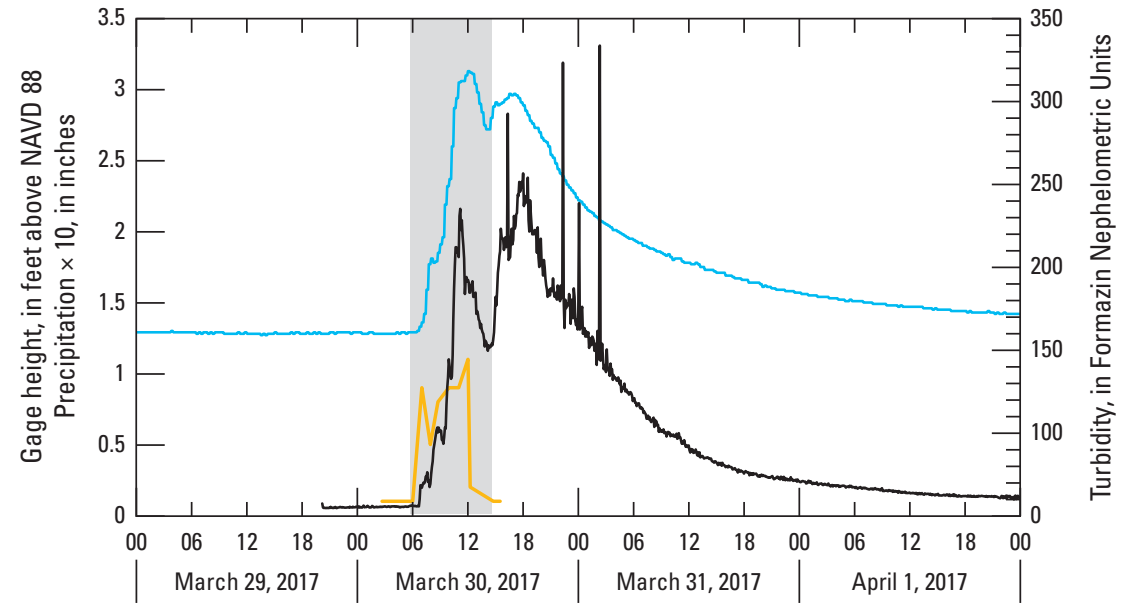

\section{Beaverdam Creek}

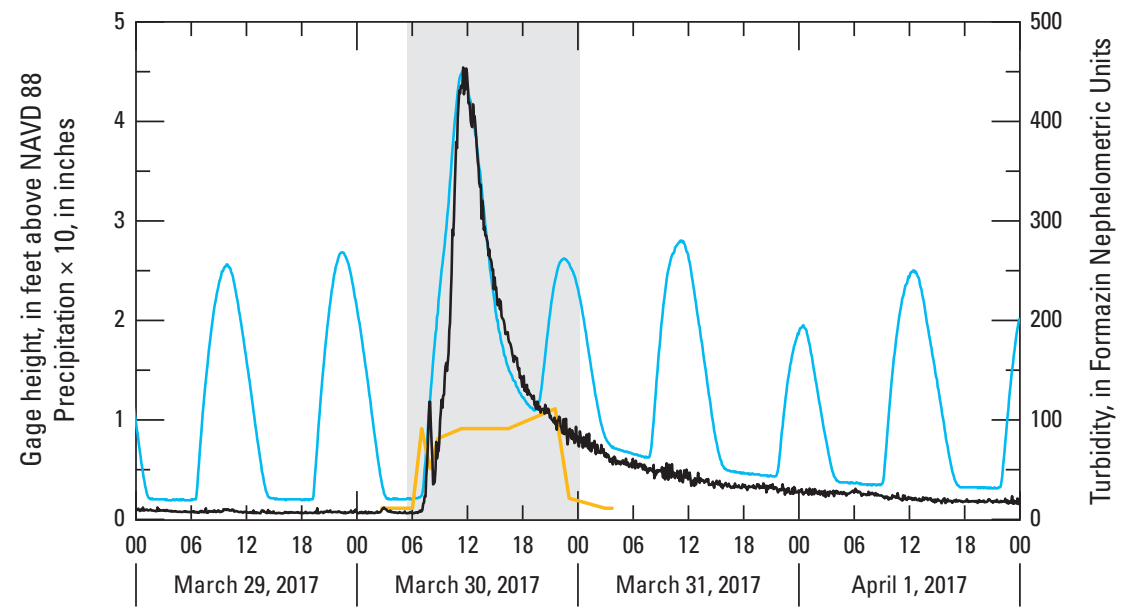

Figure 2. Stage, turbidity, and precipitation in $A$, Northeast Branch, $B$, Northwest Branch, $C$, Beaverdam Creek, D, Watts Branch, and $E$, Hickey Run during a storm from March 30 through April 2, 2017. (NAVD 88, North American Vertical Datum of 1988). 


\section{Watts Branch}
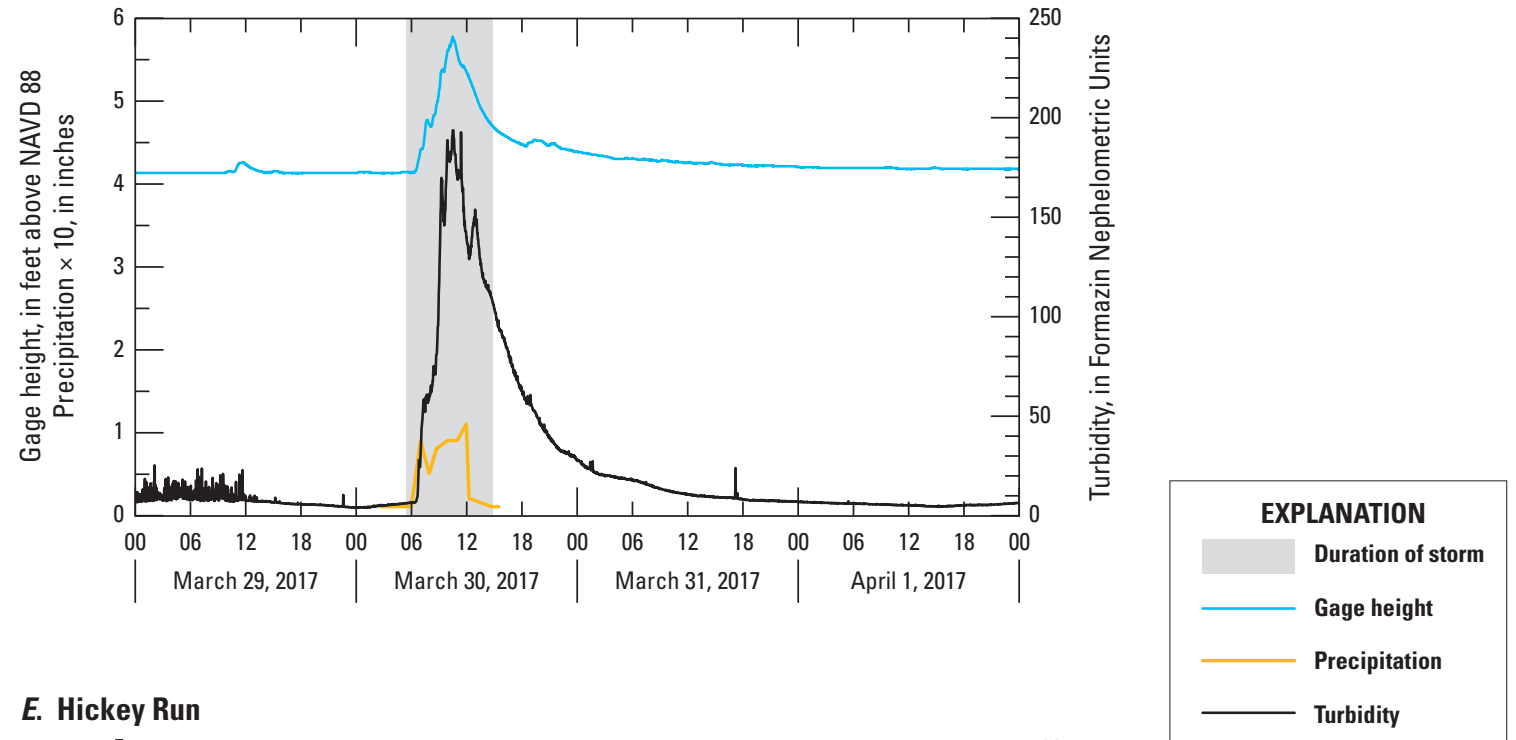

\section{E. Hickey Run}

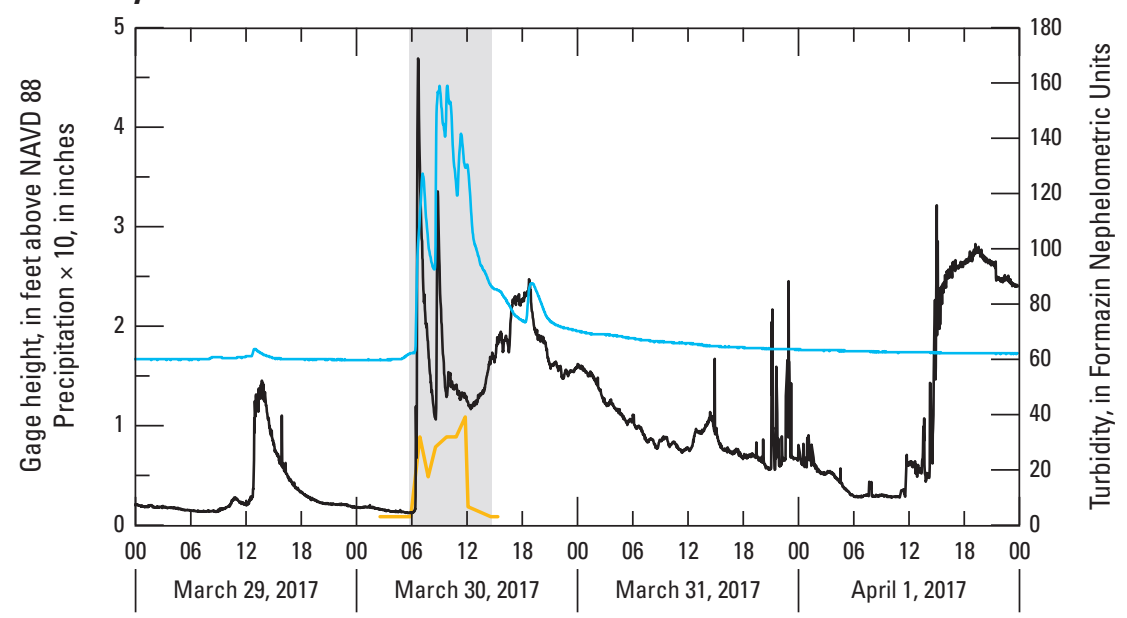

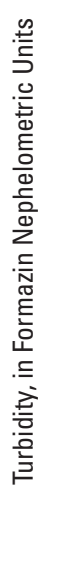

Figure 2. Stage, turbidity, and precipitation in $A$, Northeast Branch, $B$, Northwest Branch, $C$, Beaverdam Creek, $D$, Watts Branch, and $E$, Hickey Run during a storm from March 30 through April 2, 2017. (NAVD 88, North American Vertical Datum of 1988).-Continued 
number of storms were typical for the Washington, D.C., area, with no extreme events, such as hurricanes or record snowfall, occurring such as those that happened in 2016. The most relevant environmental factor, therefore, is the amount of precipitation. Table 2 summarizes the precipitation measured at Ronald Reagan Washington National Airport over the 5 years from 2013 through 2017 (data obtained from MesoWest, https://mesowest.utah.edu/). Im this 5-year period, calendar year 2017 had the second lowest total precipitation (34.7 in.) but the third highest number of days of precipitation (167). Therefore, 2017 can be considered to be a near-average year with respect to precipitation during this 5-year period.

\section{Methods}

Methods were developed to collect suspended sediment for analysis for trace levels of COCs during low-flow and storm events. Samples were collected by using automatic samplers deployed either in existing USGS gaging stations or in mobile trailers. Because detection levels for contaminants in sediment are based on having a suitable mass of sediment, the collection methods used were designed to provide a sediment mass of at least 1 gram $(\mathrm{g})$, which is needed to reach MDLs of 0.1 to 0.001 microgram per kilogram $(\mu \mathrm{g} / \mathrm{kg})$ for the COCs. Sample volume was constrained to a target volume of 60 to 70 liters $(\mathrm{L})$ of water, the maximum that could be handled safely by field crews. The sampler control routines were configured either to (1) provide flow-weighted samples of suspended sediment or to (2) maximize the amount of sediment obtained while collecting sediment during, at a minimum, the rising limb and crest of the storm hydrograph, when most of the sediment in streams is entrained and transported. The pumping rates of the autosampler for purging and sampling constrained the rate of aliquot collection and, therefore, the number of aliquots and the final composite volume that could be collected, especially in the small, flashy urban streams where water velocity varied widely. Water-quality parameters were measured continuously by using water-quality sondes deployed as part of the existing USGS monitoring network. Parameters measured included turbidity (T), specific conductance (SC), and water temperature. A precipitation monitor was deployed at Hickey Run (USGS station 01651770).

\section{Collection of Stormflow Samples}

Water samples were collected during stormflow conditions by using automated samplers (ISCO 6712), deployed either at existing gaging stations or from mobile trailers. Each station was equipped in the general configuration shown in figure 3 .

Initially (January 2017), each station was equipped with two autosamplers to collect stormwater samples at prescribed intervals. In July 2017, a third large-volume (LV) autosampler was added to collect sediment for pesticide analysis; this modification was required because of the different extraction and spiking protocols needed for the analysis for PCBs/PAHs and pesticides. Two of the autosamplers were subsequently set to collect LV (up to 70 liters [L]) composite samples, whereas the third collected individual 1-L discrete samples. Each autosampler pumped water directly from the stream through dedicated Teflon-lined polytubes (3/8-in. inside diameter, one line per sampler) held in place in the stream with steel rebar driven into the streambed and armored with a flexible polyvinyl chloride electrical conduit. The autosamplers were powered by 110 -volt line power or 12-volt marine batteries. All samplers were controlled by an electronic data logger and control unit (Campbell Scientific CR-1000x) that could be controlled through a cellphone connection, coupled with the internal control system of the autosampler. The data-logging system monitored stage and turbidity directly from the deployed data sonde, which was either located at the adjacent USGS gaging station or temporarily deployed from a mobile trailer. Sampling could be initiated and controlled by using stage, turbidity, or, when relations were available, discharge. The control program sent a signal to each autosampler at prescribed intervals to initiate the sampling program set in each autosampler. Cellphone control allowed off-site adjustment of triggering

Table 2. Summary of precipitation in the Washington, D.C., area, 2013-17.

[in., inches; data from MesoWest station KDCA, at https://mesowest.utah.edu/]

\begin{tabular}{ccccccc}
\hline Year & $\begin{array}{c}\text { Number of } \\
\text { hours having } \\
\text { precipitation }\end{array}$ & $\begin{array}{c}\text { Minimum } \\
\text { per hour } \\
\text { (in.) }\end{array}$ & $\begin{array}{c}\text { Maximum } \\
\text { per hour } \\
\text { (in.) }\end{array}$ & $\begin{array}{c}\text { Maxs having } \\
\text { precipitation }\end{array}$ & $\begin{array}{c}\text { Maximum } \\
\text { precipitation } \\
\text { per day } \\
\text { (in.) }\end{array}$ & $\begin{array}{c}\text { Yearly total } \\
\text { precipitation } \\
\text { (in.) }\end{array}$ \\
\hline 2017 & 1,014 & 0.001 & 0.71 & 167 & 2.10 & 34.7 \\
2016 & 962 & 0.001 & 0.99 & 155 & 1.17 & 31.3 \\
2015 & 601 & 0.001 & 1.42 & 125 & 2.32 & 42.2 \\
2014 & 1,122 & 0.001 & 1.11 & 163 & 3.28 & 42.9 \\
2013 & 1,185 & 0.001 & 1.33 & 169 & 2.69 & 43.5 \\
\hline
\end{tabular}




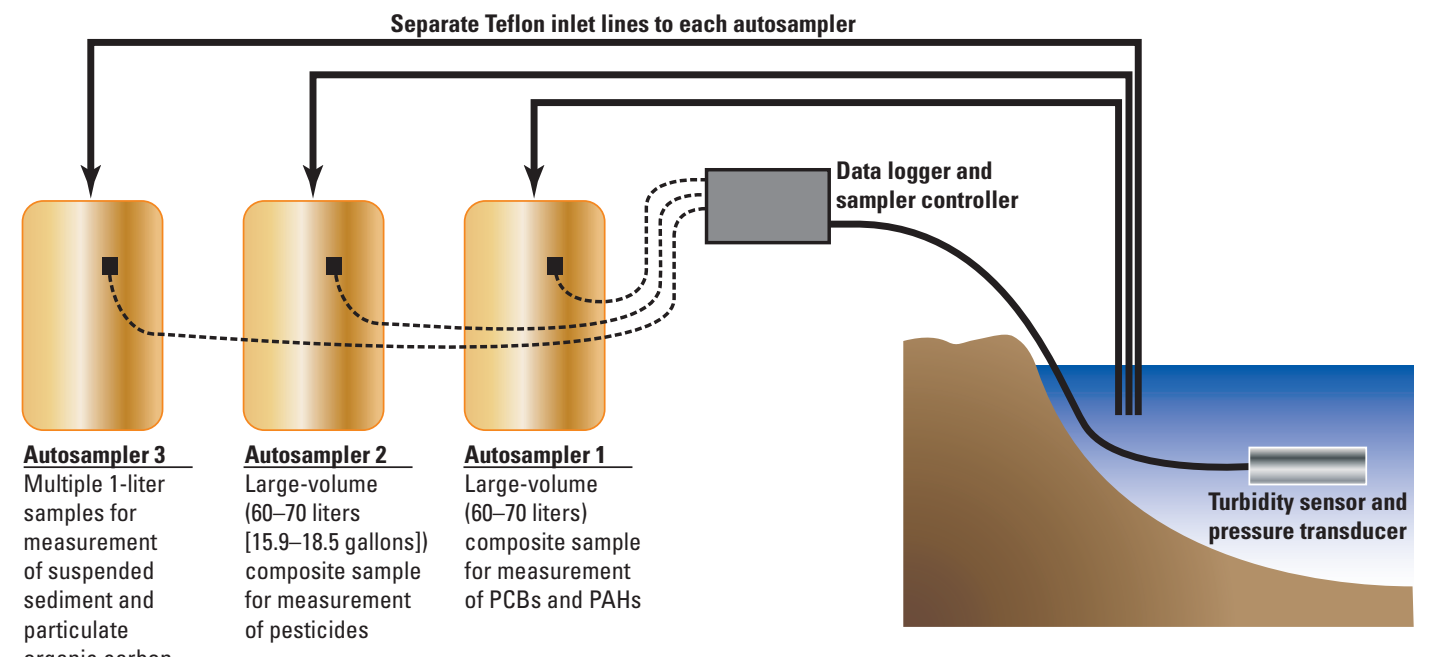

organic carbon

Figure 3. Configuration of sampling equipment used to collect large-volume and discrete samples.

parameters (stage, turbidity, or other parameter), and sampling interval (million gallons of discharge or time).

Two autosamplers were equipped to collect the LV samples, and a third autosampler was equipped to collect discrete 1-L samples for SSC/POC analysis. One autosampler was a refrigerated unit; the second (LV) and third autosamplers were nonrefrigerated units. One LV sampler was dedicated to $\mathrm{PCBs} / \mathrm{PAHs}$ and the second was dedicated to pesticides. These autosamplers were modified so they pumped directly into a large, lidded plastic bucket containing a new, unused Teflon (polytetrafluoroethylene [PTFE]) sample bag placed within two new plastic bags. The discharge tube from the autosampler was directed through the lid and directly into the PTFE bag (Fluorolab M-PTFE drum liner) that was secured around the tube by using plastic cable ties. The third autosampler held twenty-four 1-L wedge-shaped polyethylene bottles.

The autosamplers were controlled by the data-logging equipment by using the same general control program for each stream but modified for sampling intervals. For the larger NEB and NWB, where long-term discharge records were available to help in setting sampling intervals, aliquots were collected at intervals of $5 \mathrm{Mgal}$ of discharge. At Watts Branch and Hickey Run, discharge and turbidity records from 2013 showed the streams to be extremely flashy, typically with multiple peaks in stage and turbidity. As a result, different sampling controls were ultimately used in these streams. Calculations showed that it would be difficult to establish the discharge intervals required to produce a true flow-weighted sample with aliquots obtained throughout the entire storm while obtaining a suitable mass of sediment needed for low-level chemical analysis.

Trial sample collection demonstrated that aliquots would need to be collected at set time intervals for LBDC, Watts Branch, and Hickey Run. Typically, aliquots of 200 to 250 milliliters
$(\mathrm{mL})$ collected at intervals of 20 or 30 minutes would produce the 60 to $70 \mathrm{~L}$ of streamwater needed. The third autosampler at each station was configured to collect 1-L discrete samples, either concurrently with the LV sample collection or at preset time intervals. These discrete samples were analyzed for SSCs or POC in the stream throughout each storm event.

LV and discrete samples were retrieved immediately upon the conclusion of the storm events. After removing the discharge tube, the filled PTFE bag was immediately closed by using two cable ties, the two outer protection bags were tied shut, and the cover was replaced on the tub. The tub was carefully moved to a nearby van, secured, and transported to the USGS Maryland Water Science Center (Baltimore, Maryland), where it was stored in a large refrigerator. Data from each autosampler, the control unit, and the water-quality sondes were downloaded and stored.

A water-quality data sonde was deployed at each gaging station and located very near the nozzle of the inlet line for the autosamplers. The sonde was either dedicated for the site or incorporated in the adjacent USGS gaging station. A YSI 600 OMS V2 data sonde equipped with an YSI 6136 turbidity probe has been used at NWB, Watts Branch, and Hickey Run since 2003. A YSI 6920 equipped with a 6136-turbidity probe has been used at NEB since 2010. Turbidity was measured with a Campbell 501 OBS sensor at LBDC. These turbidity sensors use monochromatic near-infrared light-emitting diode light at a wavelength of 780 to 900 nanometers (nm) and a detection angle of 90 degrees ( \pm 2.5 degrees). The sondes measured turbidity, temperature, and specific conductance at intervals of either 2 or 5 minutes. Sondes at the USGS gaging stations were calibrated and maintained by USGS personnel. When deployed at the ungaged streams, the sondes were calibrated immediately before deployment following established 
USGS protocols (Wagner and others, 2006). Turbidity was calibrated by using a two-point standardization with 100- and 500-Formazin-Nephelometric-Unit (FNU) standards (Hach StablCal Standard solutions). Specific conductance was standardized to standards of 100 microsiemens per centimeter at 25 degrees Celsius $\left(\mu \mathrm{S} / \mathrm{cm}\right.$ at $25{ }^{\circ} \mathrm{C}$ ) and $500 \mu \mathrm{S} / \mathrm{cm}$ (RICCA Chemical standard solutions).

\section{Large-Volume Sample Processing}

The LV sample tubs transported to the USGS Maryland Water Science Center laboratory were stored at $4{ }^{\circ} \mathrm{C}$ in a large refrigerator until they were processed the next day. Each tub was moved carefully on a dolly to the laboratory, where the sediment was allowed to settle for a minimum of 1 hour. During this time, the sample-filtration apparatus was set up and blanks were processed.

Filtering was accomplished as follows. The outer bags and the PTFE sample bag were opened carefully and a short piece of the Teflon-lined tubing, connected to the inlet of the pump, was hand-held with the inlet 1 to 2 in. below the water surface. The pump was then started and run at approximately 100 milliliters per minute $(\mathrm{mL} / \mathrm{min})$. Water was pumped to a large-diameter (293-millimeter [mm]) stainless-steel plate filter holder. After passing through the filter, the filtered water was collected in a second plastic tub placed on a digital scale sitting on the floor, allowing the weight and volume of water processed to be determined.

Before the water was filtered, a glass-fiber filter (Advantec GF-75 GF filters, 293-mm diameter, nominal pore size 0.5 micron $[\mu \mathrm{m}]$ ) was placed by using forceps onto a clean 12-square piece of aluminum foil set on a pan balance. The filter was weighed to $0.01 \mathrm{~g}$ on a digital balance and then immediately transferred to the plate filter, the filter was assembled and tightened, and an air vent was opened on the top plate. The pump was started, forcing water into the filter holder until all air was expelled from the filter vent, which was then closed. The pump was run until it became apparent (by sound) that the filter had become clogged. As pumping was underway, the laboratory technician held the inlet tubing just below the water surface in the bagged sample. Once the filter became clogged, the pump was shut off, and the outlet line from the plate filter was then connected to a second peristaltic pump. This pump was started and run for about 5 minutes to remove as much of the water as possible from the loaded filter. The filter holder was then opened, and if the sediment appeared to still contain a sheen of water, the outlet pump was restarted until the sediment appeared to be dry. At that point, the filter was folded into quarters and returned to the foil sheet used to preweigh the filter, and the wet weight was recorded. The filter was then wrapped in the foil and placed into a plastic zip-lock bag and stored in the freezer. The filtering process was repeated until all water was removed from the PTFE sampling bag, which typically required four to six filters per sample. All foil-wrapped filters were combined in a zip-lock bag and stored frozen. Once all the native water had been removed from the bag, $4 \mathrm{~L}$ of ultrapure laboratory-grade water (Millipore OmniSolv Spectrophotometric Grade) was slowly poured down the sides of the PTFE bag and allowed to pool in the bottom. This water was suctioned through a final clean filter. The rinse was repeated until no sediment was visible in the bag. The final rinse filter was combined with the loaded filters used for the sample and stored frozen. If a second sample from the same site was awaiting processing (for pesticide analysis), a solution of warm tap water and laboratory-grade detergent was suctioned through the lines and filter holder, followed by rinses of tap water and laboratory-grade deionized water. If the length of inlet tubing showed any discoloration, it was replaced with a section of new, precleaned tubing. Typically, the two LV samples from each site were processed each day. Between collection of samples from different stations, the filter holder was scrubbed with soapy water by using a soft brush, rinsed with deionized water, then rinsed with chromatographic-grade methanol. Between sites, the sampling and pump tubing were replaced with new, precleaned tubing. Pump and transfer tubing were prewashed with a hot, soapy solution, tap water, and deionized water rinses, soaked in 5-percent hydrochloric acid solution, rinsed again in deionized water, rinsed with laboratory-grade methanol recirculated for 30 minutes, and then rinsed a final time with deionized water. Tubing was stored in sealed plastic bags.

An equipment blank was collected with each batch of filtered samples. This blank was prepared by pumping $4 \mathrm{~L}$ of ultrapure laboratory-grade (OmniSolv) water through the filtering line, with an unused glass-fiber filter placed in the holder. Once the sample was processed, the wet filter was reweighed, wrapped in foil, placed in a zip-lock bag, and stored frozen identical to the methods used to process field samples.

Throughout the project, test samples were prepared to determine a representative percent moisture in the LV samples. The percent moisture is required to convert the "wet" sample mass (sum of weights of combined filters containing wet sediment) to dry sediment mass in order to calculate chemical concentration on a per-dry-mass basis. Tests consisted of placing several hundred grams of streambed sediment into a plastic bag and adding filtered streamwater obtained during the processing of LV field samples. The sediment was then processed in a manner identical to the field samples. Typically, three filters loaded with wet sediment were obtained. The wet filters were weighed, placed in open foil packs, and dried at 110 degrees Celsius $\left({ }^{\circ} \mathrm{C}\right)$ in a laboratory oven for a minimum of 24 hours. The filter packs were then cooled in a desiccator and reweighed, and the percent moisture in the wet filters was calculated. Ten test samples were prepared over the course of the project. The average percent moisture in wet-sedimentladen filters was 66 percent ( \pm 8.5 percent). Therefore, a 70 -percent moisture content was used to provide the laboratory with the estimated dry sediment weight. (The implications of estimating dry sediment mass are discussed farther on in this report.) 


\section{Sampling Methods for Low-Flow Conditions}

Sampling during low-flow (LF) conditions was accomplished by using streamside filtration. Two peristaltic pumps were used to withdraw stream water for the two filtering lines through the same intake lines used for stormflow sampling. Two samples were required, one for analysis for $\mathrm{PCBs} / \mathrm{PAHs}$ and the second for analysis for pesticides. The pumped water was first passed through a 4-in.-long, 0.7- $\mu \mathrm{m}$ nominal glassfiber canister filter. The filtered water was then passed through a 293-mm flat-plate filter (Advantec GF-75 GF filter, 293$\mathrm{mm}$ diameter, $0.7-\mu \mathrm{m}$ nominal pore size), the same filter type used to process the LV storm samples. The filtered water was then collected in large plastic tubs that were weighed to 0.01 $\mathrm{g}$ on an electronic balance. Periodically, the flat filters would clog, as indicated by an increase in pressure in the water line. The pumps were stopped, the plate filter was opened, and the loaded filter was folded into quarters and immediately placed on a square of aluminum foil. The flat filter was replaced, and the pump restarted. The folded filter packs were stored on ice, combined with the canister filter in a zip-lock bag, and labeled. The filters from the two lines remained separate and were assigned the volume of water processed through the lines. A target volume of 1,000 L of water was used so that if a SSC of 1 milligram per liter $(\mathrm{mg} / \mathrm{L})$ was assumed, the target of $1 \mathrm{~g}$ of sediment would be obtained; typically, obtaining this volume of sediment required 5 to 6 hours of pumping. Discrete samples of stream water were collected periodically during the day, either directly from the pump outflow or by dipping a plastic sample bottle into the stream near the intake of the sampling lines. These samples were collected in pairs, one for analysis for SS and the second for analysis for POC. When the field crew returned to the laboratory, the samples were stored under refrigeration until they were delivered to the analytical laboratory. The analytical laboratory required a sediment mass for each low-flow sample. This mass was calculated by using the average SSC in the grab samples collected during streamside filtering multiplied by the total volume of water processed. Because SSCs are provided as mass on a dry-weight basis, a correction for water content in the LF filters was not required.

For each batch of LF samples collected, field equipment blanks were prepared in the field by pumping $4 \mathrm{~L}$ of ultrapure laboratory-grade water (OmniSolv) through the sampling line, a canister filter, and a flat filter. The filters were removed, wrapped in foil, and combined in a zip-lock bag, and stored frozen with the field samples. Additionally, unused canister/ flat filters were submitted for analysis twice during the study; these were identified as canister blanks.

Water samples to be analyzed for metals were collected directly into 4-L precleaned large-mouth bottles (Eagle Pitcher, class A certified clean). New, acid-rinsed pump and distributor tubing were used, and any metal fittings on the autosampler were replaced with thick-walled Teflon tubing. A field blank was prepared for each station by leaving an open, wide-mouth Teflon bottle in the autosampler chamber base during the interval when field samples were collected. When the samples were retrieved, bottles were capped with Teflon-lined plastic caps, placed on ice, and transported to the USGS Maryland Water Science Center field laboratory where they were stored under refrigeration until they were shipped. These samples required no special handling in the laboratory. Because the laboratory could remove, dry, and weigh the sediment that was analyzed for metals, it was not necessary to estimate the mass of sediment in each field sample.

\section{Bed-Sediment Sampling}

Samples of streambed sediment were retrieved after each storm event for comparison with the chemistry of the SS in the streams. Bed sediment was collected after the streams had returned to low-flow conditions, typically 1 to 2 weeks after each sampled storm event. A site was selected in each stream where a depositional area (a "velocity shadow") would likely exist; sites were typically downstream from large objects on the streambed, such as boulders or tree stumps. Samples were collected by using a precleaned stainless-steel long-handled scoop, adapted from the methods outlined in Radtke (2005). Samples consisted of the upper 1 to 2 in. of material that was carefully scooped up and poured directly into a precleaned glass sample jar equipped with a Teflon-lined lid. The sediment was allowed to settle, and the excess water was slowly decanted. Three samples (about $250 \mathrm{~mL}$ in volume) were obtained from within an area of 3 square feet. The collected material was typically "fluffy," noncompact, and dark silty clay, containing about 10 percent (by observation) sand-sized particles. Once a location was established, it was marked for revisiting. The samples were labeled and stored on ice for transport back to the USGS Maryland Water Science Center laboratory, where they were stored frozen until they were shipped to the analytical laboratory. At the laboratory, the three samples were homogenized and a mass of approximately $30 \mathrm{~g}$ was subsampled for analysis.

\section{Sampling for Suspended Sediment and Particulate Organic Carbon}

Samples for SS and POC analysis were collected throughout the storm and low-flow events for use in developing relations among turbidity, SSC, and discharge, and for determining the relation between SSC and POC concentration. Therefore, the samples were selected on the basis of the turbidity in the stream at the time of collection, thereby maximizing the range of turbidity in the dataset for each stream. POC processing was performed by using standard USGS methods (U.S. Geological Survey, 2012).

Samples were collected in 1-L precleaned plastic autosampler bottles filled either by using the autosamplers during storms or by hand during low flow. The capped bottles were labeled with the site name and the date and time of collection, then stored in coolers for transport to the USGS Maryland 
Water Science Center laboratory, where they were stored under refrigeration until they were processed, typically within 2 to 4 days of collection.

The field information (stage, discharge, turbidity, and specific conductance) was then plotted and pairs of samples were selected, one sample for SS analysis and one for POC analysis. Typically, three to eight sample pairs were selected. Samples were selected to cover the range of turbidity measured during the storm, but also to include samples from the rising and falling limbs and the crest of the hydrograph. The individual samples in each pair were selected so that the turbidities associated with the SS sample and its companion POC sample would be nearly identical; the samples typically were collected sequentially during the event.

Each sample collected for SS analysis was inspected for leakage and label information before being shipped to the USGS Kentucky Water Science Center sediment laboratory (Louisville, Kentucky). SS was measured by using standard gravimetric methods (Shreve and Downs, 2005). Samples for POC analysis were prepared by using standard USGS filtering procedures (U.S. Geological Survey, 2012). The sample was first shaken vigorously, then three 40 - to $50-\mathrm{mL}$ aliquots of water were suctioned through $25-\mathrm{mm}$ prebaked glass-fiber filters. The filtered water was collected in a graduated cylinder and the volume was measured. Each loaded filter pad was folded in half and placed on a baked 6-in.-square piece of aluminum foil, folded, and placed in a small twirl-pack plastic bag, sealed, and labeled with sample identification and volume of filtered water. The three filters used for each sample were combined into a single zip-lock bag and stored frozen until they were shipped to the USGS National Water Quality Laboratory in Denver, Colorado, where POC concentrations were measured by using a combustion method (Shreve and Downs, 2005).

\section{Chemical Analysis of Sediment}

The sediment samples, collected on flat and canister filters, were sent for analysis to TestAmerica, Inc., in Knoxville, Tennessee, and Pittsburgh, Pennsylvania. The analytical methods are referenced in table 3 , and the constituents measured are listed in table 4.

Apart from the extraction methods, analytical procedures were identical to those used in the Anacostia River Phase I Remedial Investigation (TetraTech, 2016). The use of consistent analytical methodology allows for comparison of the data collected in this study with those collected during the Phase I study. Because the samples represented sediment-laden filters, several modifications were made to the standard extraction procedures used for analysis of bed sediment. These extraction and cleanup methods were first used during the USGS sampling program conducted in the Passaic and Raritan Rivers in New Jersey in 1999 (Wilson and Bonin, 2007, 2008). The methods allowed the canister and associated flat filters for each sample to be placed directly into a large Soxhlet extraction vessel. Because the number of filters was large, several 24-hour extractions (using the sample-extraction solvents) were required for each sample. Extraction solvents (a mixture of acetone and hexane) were adjusted to allow the solvent to be split into two aliquots, one for PCB analysis and the other for PAH analysis. The analytical methods used to measure concentrations of PCB and PAH compounds are based on the use of radiolabeled internal standards and included both extraction standards and calibration standards that were added throughout the extraction process. These internal standards are required for the high-resolution gas chromatography/ high-resolution mass spectrometry (HRGC/HRMS) analysis for the individual PCB congeners by U.S. Environmental Protection Agency (EPA) method 1668A and the individual PAH compounds (EPA method SW-3540 and a TestAmerica, Inc., proprietary method based on EPA method 8270C LL). Unfortunately, the extraction procedure could not provide a suitable extract needed to determine concentrations of organochlorine pesticides. Therefore, a second LV sample dedicated for pesticide analysis was added to each stormflow and lowflow sampling event. These samples were extracted in the same manner as the PCB/PAH samples, except that appropriate internal standards were substituted for these compounds. Sample extracts were analyzed for pesticides using standard EPA methods 8081B and SW3540C. Bed-sediment samples were extracted and analyzed for PCBs/PAHs and pesticides by using the same methods used in the Phase I investigation (TetraTech, 2016) and required no modification of the extraction and analysis procedures. Bed sediments were analyzed for two suites of PAHs/volatile organic compounds (EPA methods $3540 \mathrm{C}$ and 3541), the individual PCB congeners (EPA method 1668A) and PCB aroclors (EPA method 8082A), and dioxins (EPA method 1613B). (Aroclors and dioxins are not discussed in this report.) Metals in suspended sediment and bed sediment were determined by using EPA method SW3050B. Data validation was conducted by TetraTech following EPA III guidelines, as described in the Phase I report (TetraTech, 2016).

Table 3. Analytical methods used to measure concentrations of polychlorinated biphenyls, polycyclic aromatic hydrocarbons, organochlorine pesticides, and metals in suspended and bed sediment from tributaries to the Anacostia River, Washington, D.C., 2017.

[PCB, polychlorinated biphenyl; PAH, polycyclic aromatic hydrocarbon; EPA, U.S. Environmental Protection Agency]

\begin{tabular}{ll}
\hline \multicolumn{1}{c}{ Chemical suite } & \multicolumn{1}{c}{ EPA Method } \\
\hline PCB congeners & $1668 \mathrm{~A}$ \\
PAH nonalkylated species & SW3541 \\
PAH alkylated species & SW3540C and 8270C LL \\
Organochlorine pesticides & $8081 \mathrm{~B}$ \\
Metals & SW3050B \\
PCB aroclors & $8082 \mathrm{~A}$ \\
\hline
\end{tabular}




\section{Sediment and Chemical Contaminant Loads in Tributaries to the Anacostia River, Washington, D.C., 2016-17}

Table 4. Chemical analytes measured in suspended and bed sediment in tributaries to the Anacostia River, Washington, D.C., 2017.

[CAS, Chemical Abstract Number; PCB, polychlorinated biphenyl; PAH, polycyclic aromatic hydrocarbon]

\begin{tabular}{|c|c|c|c|}
\hline \multicolumn{2}{|r|}{$\begin{array}{c}\text { CAS or laboratory } \\
\text { identifier }^{2}\end{array}$} & \multicolumn{2}{|r|}{$\begin{array}{c}\text { CAS or laboratory } \\
\text { identifier }^{2}\end{array}$} \\
\hline PCB-1 & $2051-60-7$ & PCB-57 & $70424-67-8$ \\
\hline PCB-3 & $2051-62-9$ & PCB-59/62/75 & TTNUS816 \\
\hline PCB-4 & TTNUS524 & PCB-60 & $33025-41-1$ \\
\hline PCB-5 & $16605-91-7$ & PCB-61/70/74/76 & TTNUS817 \\
\hline PCB-7 & $33284-50-3$ & PCB-64 & $52663-58-8$ \\
\hline PCB-8 & $34883-43-7$ & PCB-66 & $32598-10-0$ \\
\hline PCB-9 & $34883-39-1$ & PCB-67 & $73575-53-8$ \\
\hline PCB-10 & $33146-45-1$ & PCB-68 & $73575-52-7$ \\
\hline PCB-11 & $2050-67-1$ & PCB-72 & $41464-42-0$ \\
\hline PCB-12/13 & TTNUS800 & PCB-77 & $32598-13-3$ \\
\hline PCB-18/30 & TTNUS616 & PCB-82 & $52663-62-4$ \\
\hline PCB-19 & $38444-73-4$ & PCB-83/99 & TTNUS863 \\
\hline PCB-20/28 & TTNUS519 & PCB-84 & $52663-60-2$ \\
\hline PCB-21/33 & TTNUS810 & PCB-85/116/117 & TTNUS799 \\
\hline PCB-22 & $38444-85-8$ & PCB-86/87/97/109/119/125 & TTNUS941 \\
\hline PCB-23 & $55720-44-0$ & PCB-88/91 & TTNUS819 \\
\hline PCB-24 & $55702-45-9$ & PCB-89 & $73575-57-2$ \\
\hline PCB-25 & $55712-37-3$ & PCB-90/101/113 & TTNUS619 \\
\hline PCB-26/29 & TTNUS811 & PCB-92 & $52663-61-3$ \\
\hline PCB-27 & $38444-76-7$ & PCB-93/100 & TTNUS864 \\
\hline PCB-31 & $16606-02-3$ & PCB-94 & $73575-55-0$ \\
\hline PCB-41/40/71 & TTNUS813 & PCB-202 & $2136-99-4$ \\
\hline PCB-42 & $36559-22-5$ & PCB-203 & $52663-76-0$ \\
\hline PCB-43/73 & TTNUSA51 & PCB-204 & $74472-52-9$ \\
\hline PCB-44/47/65 & TTNUS618 & PCB-205 & $74472-53-0$ \\
\hline PCB-45/51 & TTNUS814 & PCB-206 & $40186-72-9$ \\
\hline PCB-46 & $41464-47-5$ & PCB-207 & $52663-79-3$ \\
\hline PCB-48 & $70362-47-9$ & PCB-208 & $52663-77-1$ \\
\hline PCB-49/69 & TTNUS818 & PCB-209 & 2051-24-3 \\
\hline PCB-50/53 & TTNUS815 & \multicolumn{2}{|c|}{$\mathrm{PAH}$} \\
\hline PCB-52 & $35693-99-3$ & Acenaphthene & $83-32-9$ \\
\hline PCB-54 & $15968-05-5$ & Acenaphthylene & $208-96-8$ \\
\hline PCB-55 & $74338-24-2$ & Anthracene & $120-12-7$ \\
\hline PCB-56 & $41464-43-1$ & Benzo(a)anthracene & $56-55-3$ \\
\hline
\end{tabular}


Table 4. Chemical analytes measured in suspended and bed sediment in tributaries to the Anacostia River, Washington, D.C., 2017.-Continued

[CAS, Chemical Abstract Number; PCB, polychlorinated biphenyl; PAH, polycyclic aromatic hydrocarbon]

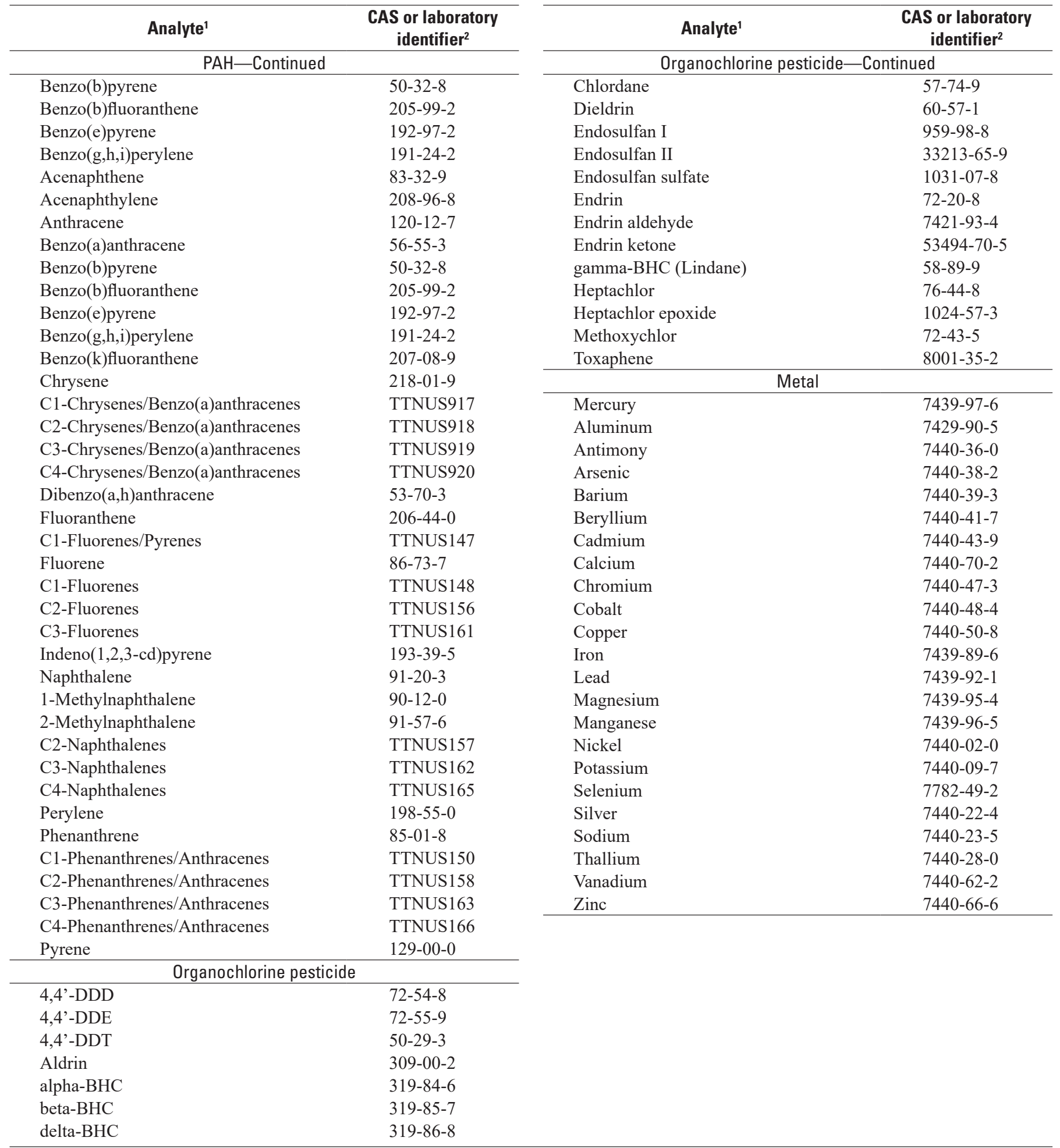

${ }^{1}$ Polychlorinated biphenyl analytes are named using International Union of Pure and Applied Chemistry nomenclature, as presented in Rigaudy and Klesney (1979).

${ }^{2}$ Identifiers indicate Chemical Abstract Services nomenclature or TTNUS, a proprietary method of the analytic laboratory based on U.S. Environmental Protection Agency method 8270C. 

study:

With respect to the analytical data generated during this

1. The laboratory was provided an estimated dry sediment weight for each LV storm sample sent for analysis. As described previously, several test samples showed an average moisture content of 70 percent ( \pm 8.5 percent) in the loaded filter pads used to estimate dry sediment weight. For the LF samples, the mass of sediment supplied for analysis was estimated as the volume of water filtered multiplied by the average SSC, which is the average dry weight of sediment in the volume of water.

2. The uncertainty in moisture content produces uncertainty in the reported contaminant concentrations. As an example, if a sample with a wet sediment weight of $166.6 \mathrm{~g}$ (sum of net wet filter weights measured in the laboratory) consists of 70 percent moisture and 30 percent sediment, and if the concentration of PCB recovered from the reported $50 \mathrm{~g}$ (30 percent multiplied by $166.6 \mathrm{~g}$ ) estimated dry weight is 10 micrograms $(\mu \mathrm{g})$, the reported concentration would be 0.2 microgram per gram $(\mu \mathrm{g} / \mathrm{g})$. If the percent moisture is 78.5 percent $( \pm 8.5$ percent), the estimated dry weight would be $35.8 \mathrm{~g}$, resulting in a concentration of $0.28 \mu \mathrm{g} / \mathrm{g}$. If the percent moisture is 61.5 percent ( \pm 8.5 percent), the estimated dry weight would be $64 \mathrm{~g}$ and the resulting concentration would be $0.16 \mu \mathrm{g} / \mathrm{g}$. This uncertainty should be added to the uncertainty inherent in the analytical measurements (approximately \pm 5 percent) and in the sediment load (unknown) when considering the contaminant loads reported here.

3. The entire suite of 209 individual PCB congeners is not resolved by the analytical methods because several coelutions occur (table 4). Throughout this study, the analytical laboratory monitored the PCB data to ensure that these coelutions remained stable. The coelutions were found to be identical to those in the Phase I study (TetraTech, 2016). Although coelutions always occur within the same homolog group of the PCBs, their presence affects some evaluation of the chemical data-for example, when attempting to determine which PCB is present in the highest concentration.

4. The internal standard methods used to resolve the various PCB and PAH compounds results in MDLs that are unique for each compound and each sample. Generally, the MDLs remained consistent among the samples, but some variation in MDLs was encountered with the PAH and pesticide groups. These changes limit the comparisons that can be made among some of the samples collected and constituent concentrations measured in this study and limits some comparisons with the results of the Phase I study (TetraTech, 2016).

\section{Chemical Results}

The chemical concentrations reported by the laboratory are in units of mass per unit of dry weight and require evaluation for potential bias before use in loading calculations. This evaluation involved correcting concentrations for possible effects originating in sample collection and preparation, as demonstrated by quality-assurance/quality-control (QA/QC) sample analyses and by comparing MDLs. Summaries of the $\mathrm{PCB}, \mathrm{PAH}$, and pesticide concentrations in blanks and environmental samples, sampling metrics, and mass loadings for the sampled storms are presented in appendixes 1 and 2 . All laboratory analytical results for concentrations of $\mathrm{PCBs}$, PAHs, pesticides and metal concentrations measured in suspended sediment and bed sediment samples as well as in field and equipment blank samples are available from the DOEE. Refer to appendix 2 for more information regarding the chemical data.

Concentrations of suspended sediment and POC along with the continuous water-quality data and discharge data used in this report are available at the USGS National Water Information System (NWIS) database (U.S. Geological Survey, 2019), which can be accessed at https://waterdata.usgs.gov/ $\mathrm{md} /$ nwis (see table 1 for USGS station identifiers).

\section{Quality-Assurance Results}

The chemical concentration data were first inspected for completeness and comparability among samples; this process included (1) identifying compounds in each sample that were also present in blanks, and (2) comparing the MDLs in blanks and field samples. As previously described, quality-assurance samples included canister blanks (CB, unused filters) and field and equipment blanks (FB and EB, laboratory blank water passed through filters and filtering equipment). Concentrations in each environmental sample were compared with those in their corresponding blanks. Concentrations in each field sample and associated blanks were first converted to contaminant mass by multiplying reported concentration by the mass of sediment associated with the sample. Field and equipment blanks were assigned (at the laboratory) a sample mass of $1 \mathrm{~g}$, multiplied by the number of filters used to process the sample. Any concentration in an environmental sample found to be less than twice the corresponding analyte mass in the associated blank (FB or EB) sample was removed from the dataset and was not used in calculating average concentrations.

In the 16 field and equipment blanks that were collected, a total of 88 PCB congeners were found in measurable concentrations. The blanks associated with the November 16 and December 7, 2017, samples contained the highest number of measurable PCBs (73 and 63, respectively). PCB-11 was the dominant contaminant in the blanks; the highest concentration in any of the blank samples was $0.18 \mu \mathrm{g} / \mathrm{kg}$ ). Other than PCB-11, the coelution of PCB-129/138/160/163 was found in many of the blanks, with a maximum concentration 
of $0.17 \mu \mathrm{g} / \mathrm{kg}$. The maximum number of detections of a PCB in the blank set was 15 , and included congeners PCB-31, the coelutions PCB-44/47/49, PCB-61/70/74/76, PCB86/87/97/109/119/125, PCB-110/115, and PCB-118. However, when the blank correction process was performed, few quantifiable PCBs in the field samples were removed.

For the PAH compounds, only one blank was found to contain measurable PAHs. Blank FB-LVSSD-120717 contained detectable PAH compounds; however, the concentrations in the associated sample (NWB, December 7, 2017) were sufficiently high that they were not affected. Three blanks were found to contain chrysene (maximum concentration $20 \mu \mathrm{g} / \mathrm{kg}$ ) and two contained acenaphthylene (maximum concentration $5.8 \mu \mathrm{g} / \mathrm{kg}$ ). One blank contained naphthalene at $160 \mu \mathrm{g} / \mathrm{kg}$, the highest concentration of the PAH suite found in the blank set. As was the case for PCBs, the associated sample was not affected by blank correction.

The blanks contained no measurable pesticide compounds; however, as discussed farther on in this report, MDLs for the pesticide analyses were high and therefore precluded using the blank correction process. Only a few suspendedsediment samples contained quantifiable concentrations above the MDLs. Additionally, the MDLs for constituents in blanks were often many times greater than MDLs for the associated field sample. For example, the sample collected on November 7, 2017, from NWB contained a concentration of $3.2 \mu \mathrm{g} / \mathrm{kg}$ of 4,4'-DDT with an MDL of $2.5 \mu \mathrm{g} / \mathrm{kg}$. The associated equipment blank, TS-EB-LVSSD-W-110717, contained no detectable 4,4'-DDT, but the MDL was $70 \mu \mathrm{g} / \mathrm{kg}$. Therefore, the differences between MDLs in blank and field samples precluded comparison of the samples.

The high MDLs for the pesticide analyses resulted in most species in environmental samples being reported as nondetected. This result was unexpected, as the MDLs for bedsediment samples were commonly one to two orders of magnitude lower than those for the suspended-sediment samples.

\section{Sediment Toxicity}

A toxic equivalency (TEQ) was calculated for each sample by using the PCB and PAH concentrations and the toxic equivalency factors (TEFs) listed in table 5. TEQ is calculated as

$$
T E Q=\sum\left(T E F_{n} \times C_{n}\right)
$$

where

$$
\begin{aligned}
T E Q= & \text { toxic equivalency, in micrograms per } \\
& \text { kilogram, } \\
T E F_{n}= & \text { toxic equivalency factor for compound } n, \\
& \text { and } \\
C_{n} \quad= & \text { concentration of compound } n .
\end{aligned}
$$

TEQs standardized the toxicity of the different PCB congeners to the dioxin tetrachlorodibenzo-p-dioxin, providing a measure of the overall toxicity in units of concentration (micrograms per kilogram) (see 1998 World Health Organization list of TEQs - Van den Berg and others, 2006). TEFs have been set for the coplanar PCBs in the tetra, penta, hexa, and hepta homolog groups. Similarly, TEFs have been established for a subset of the nonalkylated PAH compounds, allowing certain PAH concentrations to be normalized to the toxicity of benzo(a)pyrene and dibenzo(a, h) anthracene.

\section{Sediment-Bound Chemical Concentrations}

Table 6 summarizes the samples collected; lists the collection dates of the storm, low-flow, and bed-sediment samples; and identifies the associated blanks for each sample, the dry-weight mass of sediment collected for PCB/PAH and pesticide analysis, and the average suspended sediment and POC concentrations in the discrete samples collected during sampling. Total PCB (tPBC) concentrations in suspended sediment are presented in table 7, and those in bed sediment are listed in table 8. Total PAHs (tPAH), total alkylated and nona-

\begin{tabular}{|c|c|c|}
\hline $\begin{array}{l}\text { PCB or compound } \\
\text { identifier }\end{array}$ & PCB structure & TEF $^{1}$ \\
\hline 77 & 3,3',4,4'-Tetra-PCB & 0.0001 \\
\hline 81 & 3,4,4',5-Tetra-PCB & 0.0003 \\
\hline 105 & 2,3,3',4,4'-Penta-PCB & 0.00003 \\
\hline 114 & 2,3,4,4',5-Penta-PCB & 0.00003 \\
\hline 118 & $2,3^{\prime}, 4,4^{\prime}, 5$-Penta-PCB & 0.00003 \\
\hline 123 & $2^{\prime}, 3,4,4^{\prime}, 5$-Penta-PCB & 0.00003 \\
\hline 126 & 3,3',4,4',5-Penta-PCB & 0.1 \\
\hline 156 & 2,3,3',4,4',5-Неха-РCB & 0.00003 \\
\hline 157 & 2,3,3',4,4',5'-Неха-РCB & 0.00003 \\
\hline 167 & 2,3',4,4',5,5'-Неха-РCB & 0.00003 \\
\hline 169 & 3,3',4,4',5,5'-Неха-РCВ & 0.03 \\
\hline 189 & 2,3,3’4,4’5,5’-Hepta-PCB & 0.00003 \\
\hline Benzo(a)anthracene & -- & 0.1 \\
\hline Benzo(a)pyrene & -- & 1.0 \\
\hline Benzo(b)fluoranthene & -- & 0.1 \\
\hline Benzo(k)fluoranthene & -- & 0.01 \\
\hline Chrysene & -- & 0.001 \\
\hline Dibenzo(a,h)anthracene & -- & 1.0 \\
\hline Indeno(1,2,3-cd)pyrene & -- & 0.1 \\
\hline
\end{tabular}
kylated compounds, and total low- and high-molecular-weight

Table 5. Toxic equivalency factors for polychlorinated biphenyl and polycyclic aromatic hydrocarbon compounds.

[TEF, toxic equivalency factor; PCB, polychlorinated biphenyl; --, not applicable]

${ }^{1}$ TEF values from Van den Berg and others, 2006. 
Table 6. Sample identifiers and associated field or equipment blanks.

[LF, low-flow sample; S, stormflow sample; L, liter; g, gram; SSC, suspended-sediment concentration; PCB, polychlorinated biphenyls; PAH, polycyclic aromatic hydrocarbon; POC, particulate organic carbon; $\mathrm{mg} / \mathrm{L}$, milligrams per liter; nd, not determined; --, no sample obtained for pesticide analysis]

\begin{tabular}{|c|c|c|c|c|c|c|c|c|}
\hline $\begin{array}{l}\text { Sample } \\
\text { identifier }\end{array}$ & $\begin{array}{l}\text { Station } \\
\text { identifier }\end{array}$ & $\begin{array}{l}\text { Sample } \\
\text { date }\end{array}$ & $\begin{array}{c}\text { Flow } \\
\text { condition }\end{array}$ & $\begin{array}{l}\text { Associated blank } \\
\text { sample identifier }\end{array}$ & $\begin{array}{l}\text { Sediment } \\
\text { collected for } \\
\text { PCB/PAH }{ }^{1} \\
\text { (g) }\end{array}$ & $\begin{array}{l}\text { Sediment } \\
\text { collected } \\
\text { for } \\
\text { pesticides }^{1} \\
\text { (g) }\end{array}$ & $\begin{array}{c}\text { Average } \\
\text { SSC } \\
\text { (mg/L) }\end{array}$ & $\begin{array}{c}\text { Average } \\
\text { POC } \\
\text { (mg/L) }\end{array}$ \\
\hline \multicolumn{9}{|c|}{ Northeast Branch } \\
\hline TS -NEB-LVSSD-D-111616 & 01649500 & $11 / 16 / 16$ & LF & TS-FB-LVSSD-D-102516 & 2.0 & -- & 2.3 & nd \\
\hline TS-NEB-LVSSD-W-010317 & & $01 / 03 / 17$ & $\mathrm{~S}$ & TS-EB-LVSSD-W-010317 & 61 & -- & 213 & 7.83 \\
\hline TS-NEB-LVSSD-W-050517 & & $05 / 05 / 17$ & $\mathrm{~S}$ & TS-FB-LVSSD-W-050517 & 49 & 46 & 359 & 8.5 \\
\hline TS-NEB-LVSSD-W-102917 & & $10 / 29 / 17$ & $\mathrm{~S}$ & TS-EB-LVSSD-102917 & 56 & 45 & & 10.6 \\
\hline TS-NEB-LVSS-D-113017 & & $11 / 30 / 17$ & LF & TS-FB-LVSSD-D-120717 & 12 & 12 & 19.5 & 0.42 \\
\hline \multicolumn{9}{|c|}{ Northwest Branch } \\
\hline TS-NWB-LVSSD-D-111016 & 01651000 & $11 / 10 / 16$ & LF & TS-FB-LVSSD-D-102516 & 3.0 & -- & 3.0 & nd \\
\hline TS-NWB-LVSSD-W-010317 & & $01 / 03 / 17$ & $\mathrm{~S}$ & TS-EB-LVSSD-W-010317 & 52 & -- & 179 & 9.25 \\
\hline TS-NWB-LVSSD-W-072817 & & $07 / 28 / 17$ & $\mathrm{~S}$ & TS-EB-2-LVSSD-W-072817 & -- & 81 & 869 & 22.4 \\
\hline TS-NWB-LVSSD-W-110717 & & $11 / 07 / 17$ & $\mathrm{~S}$ & TS-EB-LVSSD-W-110717 & 30 & 28 & -- & -- \\
\hline TS-NWB-LVSSD-D-120717 & & $12 / 07 / 17$ & LF & TS-FB-LVSSD-D-120717 & 1.7 & 1.9 & 2.7 & 0.38 \\
\hline \multicolumn{9}{|c|}{ Beaverdam Creek } \\
\hline TS-LBC-LVSSD-D-102516 & 01651730 & $10 / 25 / 16$ & LF & TS-FB-LVSSD-D-102516 & 3.0 & -- & & \\
\hline TS-LBC-LVSSD-W-010317 & & $01 / 03 / 17$ & $\mathrm{~S}$ & TS-FB-LVSSD-W-010317 & 230 & -- & 221 & 6.85 \\
\hline TS-LBC-LVSSD-W-012317 & & $01 / 23 / 17$ & $\mathrm{~S}$ & TS-EB-LVSSD-W-012317 & 64 & -- & 399 & 15.5 \\
\hline TS-LBC-LVSSD-W-033117 & & $03 / 31 / 17$ & $\mathrm{~S}$ & TS-EB-LVSSD-W-033117 & 54 & -- & 391 & 15.1 \\
\hline TS-LBC-LVSSD-W-040617 & & $04 / 06 / 17$ & $\mathrm{~S}$ & No blank & 114 & -- & 893 & 27.1 \\
\hline TS-LBC-LVSSD-W-050517 & & $05 / 05 / 17$ & $\mathrm{~S}$ & TS-FB-LVSSD-W-050517 & 79 & 57 & 518 & 5.85 \\
\hline TS-LBC-LVSSD-D-101817 & & $10 / 18 / 17$ & $\mathrm{LF}$ & TS-FB-LVSSD-D-101817 & 4.0 & 3.0 & 3.5 & 0.29 \\
\hline TS-LBC-LVSSD-W-102917 & & $10 / 29 / 17$ & $\mathrm{~S}$ & TS-EB-LVSSD-102917 & 58 & 35 & 134 & 5.98 \\
\hline \multicolumn{9}{|c|}{ Hickey Run } \\
\hline TS-HR-LVSSD-D-111716 & 01651770 & $11 / 17 / 16$ & LF & TS-FB-LVSSD-D-102516 & 5.5 & -- & 5.0 & nd \\
\hline TS-HR-LVSSD-W-012317 & & $01 / 23 / 17$ & SS & TS-EB-LVSSD-W-012317 & 49 & -- & 352 & 27.5 \\
\hline TS-HR-LVSSD-W-033117 & & $03 / 31 / 17$ & $\mathrm{~S}$ & TS-EB-LVSSD-W-033117 & 45 & -- & 171 & 13.2 \\
\hline TS-HR-LVSSD-W-050517 & & $05 / 05 / 17$ & $\mathrm{~S}$ & TS-FB-LVSSD-W-050517 & 57 & 63 & 280 & 15.8 \\
\hline TS-HR-LVSSD-D-072717 & & $07 / 27 / 17$ & $\mathrm{LF}$ & TS-EB-2-LVSSD-W-072817 & 2.8 & 1.8 & 174 & 7.65 \\
\hline TS-HR-LVSSD-W-102917 & & $10 / 29 / 17$ & $\mathrm{~S}$ & TS-EB-LVSSD-102917 & -- & 21 & nd & nd \\
\hline TS-HR-LVSSD-W-110717 & & $11 / 07 / 17$ & $\mathrm{~S}$ & TS-EB-LVSSD-W-110717 & 42 & 41 & 73 & 12.5 \\
\hline \multicolumn{9}{|c|}{ Nash Run } \\
\hline TS-NR-LVSSD-D-072517 & 01651740 & $07 / 25 / 17$ & LF & TS-EB-3-LVSSD-W-081217 & 3.1 & 3.4 & 7.0 & 0.27 \\
\hline TS-NR-LVSSD-W-072817 & & $07 / 28 / 17$ & $\mathrm{~S}$ & TS-EB-2-LVSSD-W-072817 & 42 & 31 & 80 & 2.65 \\
\hline \multicolumn{9}{|c|}{ Pope Branch } \\
\hline TS-PB-LVSSD-D-080217 & 01651817 & $08 / 02 / 17$ & LF & TS-EB-1-LVSSD-W-080717 & 15 & 12 & 29 & nd \\
\hline TS-PB-LVSSD-W-080717 & & $08 / 07 / 17$ & $\mathrm{~S}$ & TS-EB-1-LVSSD-W-080717 & 24 & 24 & 87 & 5.26 \\
\hline \multicolumn{9}{|c|}{ Fort DuPont Creek } \\
\hline TS-FDP-LVSSD-W-081217 & 01651818 & $08 / 12 / 17$ & $\mathrm{~S}$ & TS-EB-3-LVSSD-W-081217 & 47 & 43 & 5279 & 127 \\
\hline TS-FDP-LVSSD-W-082917 & & $08 / 29 / 17$ & $\mathrm{~S}$ & TS-EB-LVSSD-W-082917 & 67 & 70 & 300 & nd \\
\hline \multicolumn{9}{|c|}{ Fort Stanton Creek } \\
\hline TS-FTS-LVSSD-D-092017 & 0165182550 & $09 / 20 / 17$ & LF & TS-FB-LVSSD-D-092017 & 3.0 & 2.4 & 4.0 & 0.49 \\
\hline TS-FTS-LVSSD-W-100917 & & $10 / 09 / 17$ & $\mathrm{~S}$ & TS-EB-LVSSD-W-100917 & 33 & 92 & 258 & nd \\
\hline
\end{tabular}

${ }^{1}$ Weights listed in this table were calculated by using 70 percent moisture content times weights of the wet-sediment-loaded filter pads. 
Table 7. Concentrations of polychlorinated biphenyls in suspended-sediment samples from tributaries to the Anacostia River.

[LF, low-flow sample; PCB, polychlorinated biphenyls; TEQ, toxic equivalency; $\mathrm{S}$, stormflow sample; $\mu \mathrm{g} / \mathrm{kg}$, micrograms per kilogram]

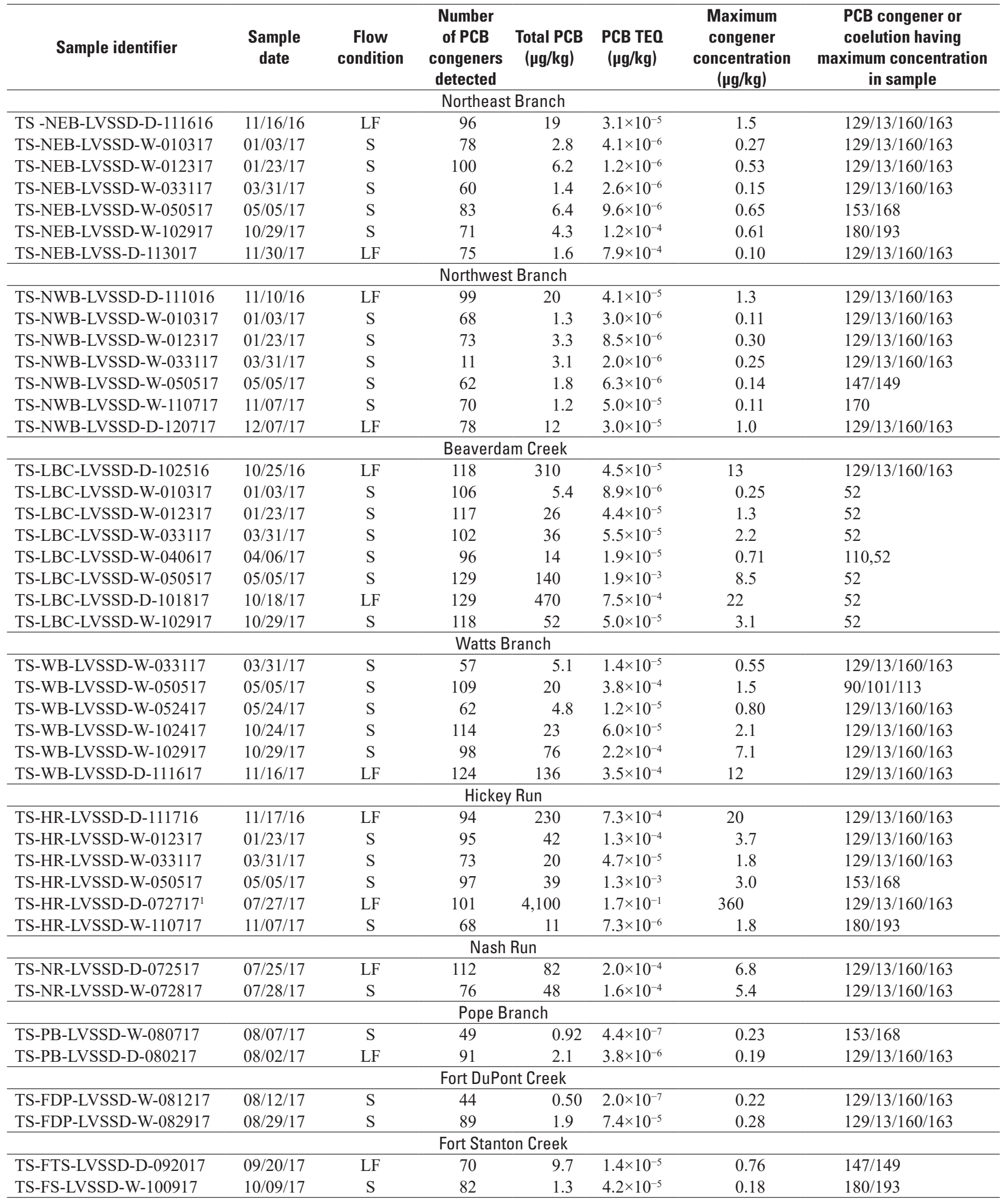


PAHs in suspended sediment and bed sediment are listed in tables 9 and 10, respectively. High-molecular-weight compounds (molecular weight greater than 202 grams per mole [g/mole]) include benzo(a)anthracene, benzo(a)pyrene, benzo(b)fluoranthene, benzo(e)pyrene, benzo(g,h,i)perylene, benzo(k)fluoranthene, chrysene, dibenzo(a,h)anthracene, indeno(1,2,3-cd)pyrene, perylene, and pyrene. Low-molecular-weight compounds include acenaphthene, acenaphthylene, anthracene, fluorene, naphthalene, 1- and 2-methylnapthalenes, and phenanthrene. Some alkylated compounds $(\mathrm{C} 1$ - to C4-chrysenes/benzo(a)anthracenes, C1-fluoranthenes/pyrenes, $\mathrm{C} 1$ - to $\mathrm{C} 3$-fluorenes, $\mathrm{C} 2$ - to $\mathrm{C} 4$-naphthalenes, and $\mathrm{C} 1$ - to
C4-phenanthrenes/anthracenes) are reported as total mixed species (for example, the C1-chrysene/benzo(a)anthracenes) because these species are not resolved by the analytical methods. The methylated compounds are of particular interest because of their toxicity (Andersson and Achten, 2015; Baird and others, 2007; Abdel-Shafy and Mansour, 2016; Flesher and Lehner, 2016).

Selected pesticide concentrations in suspended sediment and bed sediment are presented in tables 11 and 12, respectively. Concentrations of metals in suspended sediment and bed sediment are presented in tables 13 and 14, respectively.

Table 8. Concentrations of polychlorinated biphenyl compounds in samples of bed sediment from tributaries to the Anacostia River.

[ $\mu \mathrm{g} / \mathrm{kg}$, micrograms per kilogram; PCB, polychlorinated biphenyl; TEQ, toxic equivalency]

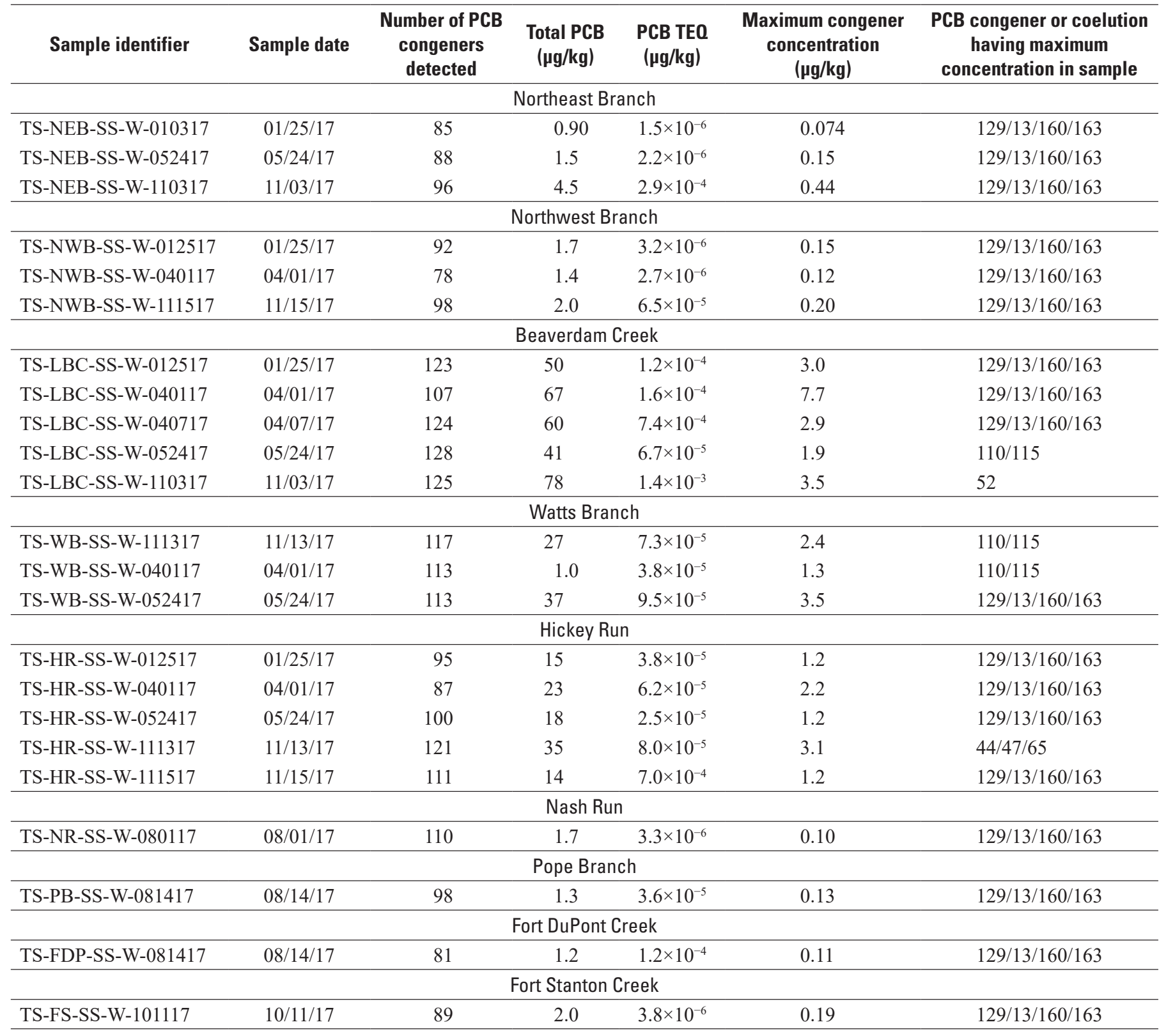


Table 9. Concentrations of polycyclic aromatic hydrocarbon compounds in suspended-sediment samples from tributaries to the Anacostia River.

[LF, low-flow sample; S, stormflow sample; $\mu \mathrm{g} / \mathrm{kg}$, micrograms per kilogram; TEQ, toxic equivalency; PAH, polycyclic aromatic hydrocarbon; BBF; benzo(b)fluoranthene; FL, fluoranthene; P, pyrene; PY, perylene; C2-P/A, C2-phenanthrenes/anthracene; C3-P/A, C3-phenanthrenes/anthracene; HMW, high molecular weight; LMW, low molecular weight]

\begin{tabular}{|c|c|c|c|c|c|c|c|c|c|c|c|c|}
\hline Sample identifier & $\begin{array}{c}\text { Sample } \\
\text { date }\end{array}$ & $\begin{array}{c}\text { Flow } \\
\text { condition }\end{array}$ & $\begin{array}{l}\text { Number } \\
\text { of PAH } \\
\text { compounds } \\
\text { detected }\end{array}$ & $\begin{array}{c}\text { Total PAH } \\
(\mu \mathrm{g} / \mathrm{kg})\end{array}$ & $\begin{array}{c}\text { Maximum } \\
\text { PAH } \\
(\mu \mathrm{g} / \mathrm{kg})\end{array}$ & $\begin{array}{l}\text { PAH compound } \\
\text { having } \\
\text { maximum } \\
\text { concentration } \\
\text { in sample }\end{array}$ & $\begin{array}{c}\text { Total } \\
\text { nonalkylated } \\
\text { PAH } \\
(\mu \mathrm{g} / \mathrm{kg})\end{array}$ & $\begin{array}{c}\text { Total HMW } \\
\text { PAH } \\
(\mu \mathrm{g} / \mathrm{kg})\end{array}$ & $\begin{array}{c}\text { Total LWM } \\
\text { PAH } \\
(\mu \mathrm{g} / \mathrm{kg})\end{array}$ & $\begin{array}{c}\text { Total } \\
\text { alkylated } \\
\text { PAH } \\
(\mu \mathrm{g} / \mathrm{kg})\end{array}$ & $\begin{array}{c}\text { Ratio of total } \\
\text { HMW/total } \\
\text { LMW }\end{array}$ & $\begin{array}{c}\text { PAH TEQ } \\
(\mu \mathrm{g} / \mathrm{kg})\end{array}$ \\
\hline \multicolumn{13}{|c|}{ Northeast Branch } \\
\hline TS -NEB-LVSSD-D-111616 & $11 / 16 / 16$ & LF & 29 & 2,700 & 290 & $\mathrm{BBF}$ & 2,000 & 1,900 & 140 & 670 & 33 & 210 \\
\hline TS-NEB-LVSSD-W-010317 & $01 / 03 / 17$ & $\mathrm{~S}$ & 23 & 1,500 & 200 & FL & 1,200 & 1,100 & 110 & 240 & 20 & 120 \\
\hline TS-NEB-LVSSD-W-012317 & $01 / 23 / 17$ & $\mathrm{~S}$ & 35 & 3,200 & 460 & FL & 2,700 & 2,400 & 240 & 530 & 20 & 240 \\
\hline TS-NEB-LVSSD-W-033117 & $03 / 31 / 17$ & $\mathrm{~S}$ & 35 & 1,100 & 170 & $\mathrm{FL}$ & 940 & 810 & 120 & 170 & 18 & 78 \\
\hline TS-NEB-LVSSD-W-050517 & $05 / 05 / 17$ & $\mathrm{~S}$ & 33 & 1,800 & 210 & FL & 1,300 & 1,200 & 110 & 490 & 38 & 150 \\
\hline TS-NEB-LVSSD-W-102917 & $10 / 29 / 17$ & $\mathrm{~S}$ & 30 & 3,200 & 340 & FL & 2,500 & 2,300 & 220 & 630 & 25 & 250 \\
\hline TS-NEB-LVSS-D-113017 & $11 / 30 / 17$ & $\mathrm{LF}$ & 23 & 384 & 44 & FL & 280 & 260 & 23 & 63 & 22 & 25 \\
\hline \multicolumn{13}{|c|}{ Northwest Branch } \\
\hline TS-NWB-LVSSD-D-111016 & $11 / 10 / 16$ & LF & 34 & 7,700 & 830 & $\mathrm{BBF}$ & 5,900 & 5,500 & 370 & 1,800 & 30 & 610 \\
\hline TS-NWB-LVSSD-W-010317 & $01 / 03 / 17$ & $\mathrm{~S}$ & 35 & 1,300 & 170 & FL & 1,100 & 900 & 140 & 270 & 26 & 90 \\
\hline TS-NWB-LVSSD-W-012317 & $01 / 23 / 17$ & $\mathrm{~S}$ & 35 & 2,100 & 240 & FL & 1,500 & 1,200 & 190 & 640 & 44 & 150 \\
\hline TS-NWB-LVSSD-W-033117 & $03 / 31 / 17$ & $\mathrm{~S}$ & 28 & 1,900 & 250 & FL & 1,400 & 1,200 & 190 & 540 & 40 & 130 \\
\hline TS-NWB-LVSSD-W-050517 & $05 / 05 / 17$ & $\mathrm{~S}$ & 35 & 1,200 & 160 & FL & 890 & 740 & 110 & 320 & 38 & 91 \\
\hline TS-NWB-LVSSD-W-110717 & $11 / 07 / 17$ & $\mathrm{~S}$ & 33 & 2,900 & 310 & FL & 2,200 & 2,100 & 170 & 680 & 31 & 240 \\
\hline TS-NWB-LVSSD-D-120717 & $12 / 07 / 17$ & LF & 25 & 6300 & 720 & $\mathrm{C} 2-\mathrm{P} / \mathrm{A}$ & 4,000 & 3,700 & 270 & 2,300 & 57 & 370 \\
\hline \multicolumn{13}{|c|}{ Beaverdam Creek } \\
\hline TS-LBC-LVSSD-D-102516 & $10 / 25 / 16$ & LF & 34 & 2,300 & 190 & BEP & 1,500 & 1,300 & 130 & 820 & 56 & 130 \\
\hline TS-LBC-LVSSD-W-010317 & $01 / 03 / 17$ & $\mathrm{~S}$ & 35 & 320 & 44 & FL & 260 & 230 & 30 & 60 & 23 & 22 \\
\hline TS-LBC-LVSSD-W-012317 & $01 / 23 / 17$ & $\mathrm{~S}$ & 35 & 1,400 & 180 & FL & 1,100 & 960 & 150 & 260 & 23 & 98 \\
\hline TS-LBC-LVSSD-W-033117 & $03 / 31 / 17$ & $\mathrm{~S}$ & 35 & 1,600 & 210 & FL & 1,300 & 1,200 & 140 & 270 & 20 & 130 \\
\hline TS-LBC-LVSSD-W-040617 & $04 / 06 / 17$ & $\mathrm{~S}$ & 35 & 930 & 150 & FL & 770 & 630 & 130 & 160 & 21 & 54 \\
\hline TS-LBC-LVSSD-W-050517 & $05 / 15 / 17$ & $\mathrm{~S}$ & 34 & 3,400 & 400 & $\mathrm{BBF}$ & 2,800 & 2,600 & 180 & 650 & 23 & 300 \\
\hline TS-LBC-LVSSD-D-101817 & $10 / 18 / 17$ & LF & 35 & 5,600 & 530 & PY & 3,900 & 3,800 & 290 & 1,400 & 36 & 440 \\
\hline TS-LBC-LVSSD-W-102917 & $10 / 29 / 17$ & $\mathrm{~S}$ & 29 & 1,900 & 220 & $\mathrm{BBF}$ & 1,600 & 1,500 & 94 & 320 & 20 & 170 \\
\hline
\end{tabular}


Table 9. Concentrations of polycyclic aromatic hydrocarbon compounds in suspended-sediment samples from tributaries to the Anacostia River.-Continued

[LF, low-flow sample; S, stormflow sample; $\mu \mathrm{g} / \mathrm{kg}$, micrograms per kilogram; TEQ, toxic equivalency; PAH, polycyclic aromatic hydrocarbon; BBF; benzo(b)fluoranthene; FL, fluoranthene; P, pyrene; PY, perylene; C2-P/A, C2-phenanthrenes/anthracene; C3-P/A, C3-phenanthrenes/anthracene; HMW, high molecular weight; LMW, low molecular weight]

\begin{tabular}{|c|c|c|c|c|c|c|c|c|c|c|c|c|}
\hline Sample identifier & $\begin{array}{l}\text { Sample } \\
\text { date }\end{array}$ & $\begin{array}{c}\text { Flow } \\
\text { condition }\end{array}$ & $\begin{array}{c}\text { Number } \\
\text { of PAH } \\
\text { compounds } \\
\text { detected }\end{array}$ & $\begin{array}{c}\text { Total PAH } \\
(\mu \mathrm{g} / \mathrm{kg})\end{array}$ & $\begin{array}{c}\text { Maximum } \\
\text { PAH } \\
(\mu \mathrm{g} / \mathrm{kg})\end{array}$ & $\begin{array}{l}\text { PAH compound } \\
\text { having } \\
\text { maximum } \\
\text { concentration } \\
\text { in sample }\end{array}$ & $\begin{array}{c}\text { Total } \\
\text { nonalkylated } \\
\text { PAH } \\
(\mu \mathrm{g} / \mathrm{kg})\end{array}$ & $\begin{array}{c}\text { Total HMW } \\
\text { PAH } \\
(\mu \mathrm{g} / \mathrm{kg})\end{array}$ & $\begin{array}{c}\text { Total LWM } \\
\text { PAH } \\
(\mu \mathrm{g} / \mathrm{kg})\end{array}$ & $\begin{array}{c}\text { Total } \\
\text { alkylated } \\
\text { PAH } \\
(\mu \mathrm{g} / \mathrm{kg})\end{array}$ & $\begin{array}{l}\text { Ratio of total } \\
\text { HMW/total } \\
\text { LMW }\end{array}$ & $\begin{array}{c}\text { PAH TEQ } \\
(\mu \mathrm{g} / \mathrm{kg})\end{array}$ \\
\hline \multicolumn{13}{|c|}{ Watts Branch } \\
\hline TS-WB-LVSSD-D-111617 & $11 / 16 / 17$ & $\mathrm{~S}$ & 33 & 2,900 & 280 & $\mathrm{P}$ & 2,100 & 19,000 & 240 & 820 & 39 & 200 \\
\hline TS-WB-LVSSD-W-033117 & $03 / 31 / 17$ & $\mathrm{~S}$ & 35 & 1,200 & 140 & FL & 910 & 790 & 110 & 290 & 32 & 80 \\
\hline TS-WB-LVSSD-W-050517 & $05 / 05 / 17$ & S & 35 & 1,400 & 150 & FL & 1,100 & 980 & 92 & 330 & 31 & 110 \\
\hline TS-WB-LVSSD-W-052417 & $05 / 24 / 17$ & $\mathrm{~S}$ & 34 & 1,000 & 100 & FL & 730 & 670 & 57 & 300 & 42 & 74 \\
\hline TS-WB-LVSSD-W-102417 & $10 / 24 / 17$ & S & 35 & 1,800 & 150 & FL & 1,200 & 1,100 & 100 & 608 & 51 & 120 \\
\hline TS-WB-LVSSD-W-102917 & $11 / 16 / 17$ & $\mathrm{~S}$ & 33 & 5,100 & 480 & $\mathrm{BBF}$ & 3,700 & 3,200 & 420 & 1,400 & 39 & 359 \\
\hline \multicolumn{13}{|c|}{ Hickey Run } \\
\hline TS-HR-LVSSD-D-111716 & $11 / 17 / 16$ & LF & 34 & 66,000 & 8,500 & $\mathrm{C} 2-\mathrm{P} / \mathrm{A}$ & 11,000 & 9,900 & 1,100 & 55,000 & 35 & 710 \\
\hline TS-HR-LVSSD-W-012317 & $01 / 23 / 17$ & $\mathrm{~S}$ & 35 & 8,700 & 730 & BBF & 5,600 & 4,800 & 750 & 3,100 & 55 & 570 \\
\hline TS-HR-LVSSD-W-033117 & $03 / 31 / 17$ & $\mathrm{~S}$ & 35 & 6,000 & 470 & $\mathrm{P}$ & 3,600 & 3,100 & 480 & 2,300 & 64 & 310 \\
\hline TS-HR-LVSSD-W-050517 & $05 / 05 / 17$ & $\mathrm{~S}$ & 35 & 4,500 & 430 & BBF & 3,200 & 2,800 & 310 & 1,300 & 40 & 350 \\
\hline TS-HR-LVSSD-D-072717 & $07 / 27 / 17$ & LF & 34 & 220,000 & 28,000 & $\mathrm{C} 3-\mathrm{P} / \mathrm{A}$ & 58,000 & 49,000 & 7,200 & 160,000 & 33 & 5,300 \\
\hline TS-HR-LVSSD-W-110717 & $11 / 07 / 17$ & $\mathrm{~S}$ & 35 & 6,000 & 410 & $\mathrm{P}$ & 3,200 & 2,800 & 400 & 2,800 & 86 & 310 \\
\hline \multicolumn{13}{|c|}{ Nash Run } \\
\hline TS-NR-LVSSD-D-072517 & $07 / 25 / 17$ & LF & 31 & 5,600 & 520 & FL & 4,000 & 3,700 & 270 & 1,700 & 14 & 389 \\
\hline TS-NR-LVSSD-W-072817 & $07 / 28 / 17$ & $\mathrm{~S}$ & 34 & 5,500 & 590 & $\mathrm{FL}$ & 4,400 & 3,900 & 400 & 1,100 & 25 & 490 \\
\hline \multicolumn{13}{|c|}{ Pope Branch } \\
\hline TS-PB-LVSSD-D-080217 & $08 / 02 / 17$ & LF & 12 & 500 & 72 & $\mathrm{C} 3-\mathrm{P} / \mathrm{A}$ & 180 & 120 & 65 & 320 & 180 & 11 \\
\hline TS-PB-LVSSD-W-080717 & $08 / 07 / 17$ & $\mathrm{~S}$ & 14 & 760 & 82 & FL & 570 & 520 & 47 & 200 & 35 & 57 \\
\hline \multicolumn{13}{|c|}{ Fort DuPont Creek } \\
\hline TS-FDP-LVSSD-W-081217 & $08 / 12 / 17$ & $\mathrm{~S}$ & 34 & 830 & 93 & $\mathrm{FL}$ & 630 & 490 & 55 & 290 & 15 & 57 \\
\hline TS-FDP-LVSSD-W-082917 & $08 / 29 / 17$ & $\mathrm{~S}$ & 35 & 380 & 43 & $\mathrm{P}$ & 300 & 260 & 34 & 84 & 28 & 30 \\
\hline \multicolumn{13}{|c|}{ Fort Stanton Creek } \\
\hline TS-FTS-LVSSD-D-092017 & $09 / 20 / 17$ & LF & 24 & 630 & 70 & $\mathrm{C} 3-\mathrm{P} / \mathrm{A}$ & 470 & 370 & 42 & 220 & 45 & 43 \\
\hline TS-FS-LVSSD-W-100917 & $10 / 09 / 17$ & $\mathrm{~S}$ & 31 & 590 & 60 & $\mathrm{P}$ & 470 & 350 & 46 & 180 & 40 & 36 \\
\hline
\end{tabular}


Table 10. Concentrations of polycyclic aromatic hydrocarbon compounds in bed sediment in tributaries to the Anacostia River.

[LF, low-flow sample; S, stormflow sample; $\mu \mathrm{g} / \mathrm{kg}$, micrograms per kilogram; TEQ, toxic equivalency; PAH, polycyclic aromatic hydrocarbon; BBF; benzo(b)fluoranthene; FL, fluoranthene; P, pyrene; PY, perylene; HMW, high molecular weight; LMW, low molecular weight]

\begin{tabular}{|c|c|c|c|c|c|c|c|c|c|c|c|}
\hline Sample identifier & $\begin{array}{l}\text { Sample } \\
\text { date }\end{array}$ & $\begin{array}{c}\text { Number } \\
\text { of PAH } \\
\text { compounds } \\
\text { detected }\end{array}$ & $\begin{array}{c}\text { Total PAH } \\
(\mu \mathrm{g} / \mathrm{kg})\end{array}$ & $\begin{array}{c}\text { Maximum PAH } \\
(\mu \mathrm{g} / \mathrm{kg})\end{array}$ & $\begin{array}{l}\text { PAH compound } \\
\text { having maximum } \\
\text { concentration in } \\
\text { sample }\end{array}$ & $\begin{array}{c}\text { Total } \\
\text { nonalkylated } \\
\text { PAH } \\
(\mu \mathrm{g} / \mathrm{kg})\end{array}$ & $\begin{array}{c}\text { Total HMW } \\
\text { PAH } \\
(\mu \mathrm{g} / \mathrm{kg})\end{array}$ & $\begin{array}{c}\text { Total LMW } \\
\text { PAH } \\
(\mu \mathrm{g} / \mathrm{kg})\end{array}$ & $\begin{array}{c}\text { Total } \\
\text { alkylated } \\
\text { PAH } \\
(\mu \mathrm{g} / \mathrm{kg})\end{array}$ & $\begin{array}{c}\text { Ratio } \\
\text { HMW/LMW }\end{array}$ & $\begin{array}{c}\text { PAH TEO } \\
(\mu \mathrm{g} / \mathrm{kg})\end{array}$ \\
\hline \multicolumn{12}{|c|}{ Northeast Branch } \\
\hline TS-NEB-SS-W-010317 & $01 / 25 / 17$ & 31 & 390 & 33 & $\mathrm{BBF}$ & 230 & 210 & 15 & 160 & 72 & 24 \\
\hline TS-NEB-SS-W-052417 & $05 / 24 / 17$ & 30 & 2,200 & 320 & FL & 1,400 & 1,400 & 25 & 800 & 57 & 52 \\
\hline TS-NEB-SS-W-110317 & $11 / 03 / 17$ & 29 & 52,000 & 7,300 & FL & 47,000 & 43,000 & 3,500 & 5,300 & 11 & 5,200 \\
\hline \multicolumn{12}{|c|}{ Northwest Branch } \\
\hline TS-NWB-SS-W-012517 & $01 / 25 / 17$ & 30 & 3,600 & 450 & FL & 2,900 & 2,700 & 220 & 700 & 24 & 330 \\
\hline TS-NWB-SS-W-040117 & $04 / 01 / 17$ & 31 & 1,200 & 140 & FL & 1,000 & 970 & 55 & 200 & 20 & 130 \\
\hline TS-NWB-SS-W-111517 & $11 / 15 / 17$ & 31 & 2,700 & 350 & FL & 2,300 & 2,100 & 180 & 430 & 19 & 270 \\
\hline \multicolumn{12}{|c|}{ Beaverdam Creek } \\
\hline TS-LBC-SS-W-012517 & $01 / 25 / 17$ & 35 & 1,300 & 140 & $\mathrm{FL}$ & 990 & 910 & 77 & 300 & 30 & 110 \\
\hline TS-LBC-SS-W-040117 & 04/01/17 & 30 & 6,200 & 910 & FL & 5,400 & 4,800 & 680 & 810 & 15 & 570 \\
\hline TS-LBC-SS-W-040717 & 04/07/17 & 32 & 2,300 & 300 & FL & 1,900 & 1,700 & 180 & 330 & 17 & 210 \\
\hline TS-LBC-SS-W-052417 & $05 / 24 / 17$ & 31 & 680 & 81 & FL & 550 & 520 & 35. & 130 & 24 & 66 \\
\hline TS-LBC-SS-W-110317 & $11 / 03 / 17$ & 31 & 2,400 & 310 & FL & 2,100 & 1,900 & 150 & 370 & 18 & 240 \\
\hline \multicolumn{12}{|c|}{ Watts Branch } \\
\hline TS-WB-SS-W-111317 & $11 / 13 / 17$ & 32 & 4,800 & 540 & FL & 3,700 & 3,300 & 350 & 1,100 & 30 & 430 \\
\hline TS-WB-SS-W-040117 & $04 / 01 / 17$ & 33 & 1,600 & 170 & FL & 970 & 920 & 34 & 370 & 30 & 150 \\
\hline TS-WB-SS-W-052417 & $05 / 24 / 17$ & 31 & 3,400 & 440 & FL & 2,800 & 2,500 & 260 & 620 & 22 & 320 \\
\hline \multicolumn{12}{|c|}{ Hickey Run } \\
\hline TS-HR-SS-W-012517 & $01 / 25 / 17$ & 34 & 4,100 & 310 & $\mathrm{P}$ & 22,000 & 2,000 & 200 & 1,900 & 86 & 240 \\
\hline TS-HR-SS-W-040117 & $04 / 01 / 17$ & 35 & 18,000 & 1,600 & $\mathrm{P}$ & 10,000 & 8,800 & 1,400 & 7,500 & 73 & 1,300 \\
\hline TS-HR-SS-W-052417 & $05 / 24 / 17$ & 33 & 13,000 & 1,800 & FL & 11,000 & 9,300 & 1,300 & 2,400 & 23 & 1,100 \\
\hline TS-HR-SS-W-111317 & $11 / 13 / 17$ & 32 & 18,000 & 3,800 & FL & 14,000 & 12,000 & 1,700 & 4,100 & 30 & 750 \\
\hline TS-HR-SS-W-111517 & $11 / 15 / 17$ & 34 & 8,900 & 850 & FL & 6,200 & 5,400 & 780 & 2,700 & 44 & 660 \\
\hline \multicolumn{12}{|c|}{ Nash Run } \\
\hline TS-NR-SS-W-080117 & 08/01/17 & 34 & 700 & 300 & PY & 560 & 530 & 27 & 140 & 26 & 30 \\
\hline \multicolumn{12}{|c|}{ Pope Branch } \\
\hline TS-PB-SS-W-081417 & $08 / 14 / 17$ & 32 & 280 & 27 & FL & 210 & 190 & 20 & 71 & 34 & 26 \\
\hline \multicolumn{12}{|c|}{ Fort DuPont Creek } \\
\hline TS-FDP-SS-W-081417 & $08 / 14 / 17$ & 35 & 4,600 & 55 & PY & 340 & 300 & 31 & 120 & 35 & 37 \\
\hline \multicolumn{12}{|c|}{ Fort Stanton Creek } \\
\hline TS-FS-SS-W-101117 & $10 / 11 / 17$ & 31 & 380 & 42 & FL & 300 & 270 & 30 & 82 & 28 & 34 \\
\hline
\end{tabular}


Table 11. Concentrations of selected pesticides in suspended-sediment samples from tributaries to the Anacostia River.

[LF, low-flow sample; S, stormflow sample; $\mu \mathrm{g} / \mathrm{kg}$, micrograms per kilogram; nd, not detected]

\begin{tabular}{|c|c|c|c|c|c|c|c|c|c|c|c|}
\hline Sample identifier & $\begin{array}{c}\text { Sample } \\
\text { date }\end{array}$ & $\begin{array}{c}\text { Flow } \\
\text { condition }\end{array}$ & $\begin{array}{l}\text { Aldrin } \\
(\mu \mathrm{g} / \mathrm{kg})\end{array}$ & $\begin{array}{c}\text { delta-BHC } \\
(\mu \mathrm{g} / \mathrm{kg})\end{array}$ & $\begin{array}{c}\text { Chlordane } \\
(\mu \mathrm{g} / \mathrm{kg})\end{array}$ & $\begin{array}{c}\text { 4,4'-DDT } \\
(\mu \mathrm{g} / \mathrm{kg})\end{array}$ & $\begin{array}{c}\text { 4,4'-DDE } \\
(\mu \mathrm{g} / \mathrm{kg})\end{array}$ & $\begin{array}{l}\text { Dieldrin } \\
\text { ( } \mu \mathrm{g} / \mathrm{kg})\end{array}$ & $\begin{array}{c}\text { Endrin } \\
\text { aldehyde } \\
(\mu \mathrm{g} / \mathrm{kg})\end{array}$ & $\begin{array}{c}\text { Heptachlor } \\
\text { epoxide } \\
(\mu \mathrm{g} / \mathrm{kg})\end{array}$ & $\begin{array}{c}\text { Methoxyclor } \\
(\mu \mathrm{g} / \mathrm{kg})\end{array}$ \\
\hline \multicolumn{12}{|c|}{ Northeast Branch } \\
\hline TS-NEB-LVSSD-W-050517 & $05 / 05 / 17$ & $\mathrm{~S}$ & 1.5 & nd & nd & nd & nd & nd & nd & nd & 1.6 \\
\hline TS-NEB-LVSSD-W-102917 & $10 / 29 / 17$ & $\mathrm{~S}$ & nd & nd & 42 & nd & nd & nd & nd & nd & nd \\
\hline TS-NEB-LVSS-D-113017 & $11 / 30 / 17$ & LF & nd & nd & nd & nd & nd & nd & nd & nd & nd \\
\hline \multicolumn{12}{|c|}{ Northwest Branch } \\
\hline TS-NWB-LVSSD-W-110717 & $11 / 07 / 17$ & $\mathrm{~S}$ & nd & nd & 21 & 3.2 & nd & nd & nd & nd & nd \\
\hline TS-NWB-LVSSD-D-120717 & $12 / 07 / 17$ & $\mathrm{LF}$ & nd & nd & nd & nd & nd & 150 & nd & nd & nd \\
\hline \multicolumn{12}{|c|}{ Beaverdam Creek } \\
\hline TS-LBC-LVSSD-W-050517 & $05 / 05 / 17$ & $\mathrm{~S}$ & nd & nd & nd & 7.5 & nd & nd & nd & nd & nd \\
\hline TS-LBC-LVSSD-D-101817 & $10 / 18 / 17$ & $\mathrm{LF}$ & nd & nd & nd & nd & nd & nd & nd & nd & nd \\
\hline TS-LBC-LVSSD-W-102917 & $10 / 29 / 17$ & $\mathrm{~S}$ & nd & nd & 35 & nd & nd & nd & nd & nd & nd \\
\hline \multicolumn{12}{|c|}{ Watts Branch } \\
\hline TS-WB-LVSSD-W-102417 & $10 / 24 / 17$ & $\mathrm{~S}$ & nd & nd & nd & nd & nd & nd & nd & nd & nd \\
\hline TS-WB-LVSSD-W-102917 & $10 / 29 / 17$ & $\mathrm{~S}$ & & 24 & 63 & nd & nd & nd & nd & nd & nd \\
\hline TS-WB-LVSSD-D-111617 & $11 / 16 / 17$ & LF & nd & nd & nd & 560 & nd & nd & 390 & nd & 410 \\
\hline \multicolumn{12}{|c|}{ Hickey Run } \\
\hline TS-HR-LVSSD-W-050517 & $05 / 05 / 17$ & $\mathrm{~S}$ & nd & nd & nd & nd & 4.6 & nd & nd & nd & nd \\
\hline TS-HR-LVSSD-D-072717 & $07 / 27 / 17$ & $\mathrm{LF}$ & nd & nd & nd & nd & nd & nd & nd & nd & nd \\
\hline TS-HR-LVSSD-W-110717 & $11 / 07 / 17$ & $\mathrm{~S}$ & nd & nd & nd & nd & nd & nd & nd & nd & nd \\
\hline \multicolumn{12}{|c|}{ Nash Run } \\
\hline TS-NR-LVSSD-D-072517 & $07 / 25 / 17$ & $\mathrm{LF}$ & nd & nd & nd & nd & nd & nd & nd & nd & nd \\
\hline TS-NR-LVSSD-W-072817 & $07 / 28 / 17$ & $\mathrm{~S}$ & nd & nd & nd & nd & nd & nd & nd & nd & nd \\
\hline \multicolumn{12}{|c|}{ Pope Branch } \\
\hline TS-PB-LVSSD-D-080217 & $08 / 02 / 17$ & LS & nd & nd & nd & nd & nd & nd & nd & nd & nd \\
\hline TS-PB-LVSSD-W-080717 & 08/07/17 & $\mathrm{S}$ & nd & nd & nd & nd & nd & nd & nd & nd & nd \\
\hline \multicolumn{12}{|c|}{ Fort DuPont Creek } \\
\hline TS-FDP-LVSSD-W-081217 & $08 / 12 / 17$ & $\mathrm{~S}$ & nd & nd & nd & nd & nd & nd & nd & nd & nd \\
\hline TS-FDP-LVSSD-W-082917 & 08/29/17 & $\mathrm{S}$ & nd & nd & nd & nd & 4.4 & nd & nd & nd & nd \\
\hline \multicolumn{12}{|c|}{ Fort Stanton Creek } \\
\hline TS-FTS-LVSSD-D-092017 & $09 / 20 / 17$ & $\mathrm{LF}$ & nd & nd & nd & nd & nd & nd & nd & nd & nd \\
\hline TS-FTS-LVSSD-W-100917 & $10 / 09 / 17$ & $\mathrm{~S}$ & nd & nd & nd & nd & nd & nd & nd & nd & nd \\
\hline
\end{tabular}


Table 12. Concentrations of selected pesticides in bed sediment in tributaries to the Anacostia River.

$[\mu \mathrm{g} / \mathrm{kg}$, micrograms per kilogram; nd, not detected]

\begin{tabular}{|c|c|c|c|c|c|c|c|c|c|c|c|}
\hline Sample identifier & $\begin{array}{l}\text { Sample } \\
\text { date }\end{array}$ & $\begin{array}{c}\text { gamma-BHC } \\
(\mu \mathrm{g} / \mathrm{kg})\end{array}$ & $\begin{array}{c}\text { Chlordane } \\
(\mu \mathrm{g} / \mathrm{kg})\end{array}$ & $\begin{array}{c}\text { 4,4'-DDD } \\
(\mu \mathrm{g} / \mathrm{kg})\end{array}$ & $\begin{array}{c}\text { 4,4'-DDT } \\
(\mu \mathrm{g} / \mathrm{kg})\end{array}$ & $\begin{array}{c}\text { 4,4'-DDE } \\
(\mu \mathrm{g} / \mathrm{kg})\end{array}$ & $\begin{array}{l}\text { Dieldrin } \\
\text { ( } \mu \mathrm{g} / \mathrm{kg})\end{array}$ & $\begin{array}{l}\text { Endrin } \\
(\mu \mathrm{g} / \mathrm{kg})\end{array}$ & $\begin{array}{l}\text { Endrin } \\
\text { aldehyde } \\
(\mu \mathrm{g} / \mathrm{kg})\end{array}$ & $\begin{array}{c}\text { Heptachlor } \\
(\mu \mathrm{g} / \mathrm{kg})\end{array}$ & $\begin{array}{c}\text { Heptachlor } \\
\text { epoxide } \\
(\mu \mathrm{g} / \mathrm{kg})\end{array}$ \\
\hline \multicolumn{12}{|c|}{ Northeast Branch } \\
\hline TS-NEB-SS-W-010317 & $01 / 25 / 17$ & nd & 7.7 & nd & nd & 0.25 & 0.46 & nd & nd & 0.17 & 0.31 \\
\hline TS-NEB-SS-W-052417 & $05 / 24 / 17$ & nd & 11 & nd & nd & nd & 0.27 & nd & nd & nd & 0.32 \\
\hline TS-NEB-SS-W-110317 & $11 / 03 / 17$ & nd & nd & nd & 0.79 & nd & 0.30 & nd & nd & nd & nd \\
\hline \multicolumn{12}{|c|}{ Northwest Branch } \\
\hline TS-NWB-SS-W-012517 & $01 / 25 / 17$ & nd & 8.1 & 0.12 & 0.11 & nd & 0.40 & nd & nd & 0.18 & 0.21 \\
\hline TS-NWB-SS-W-040117 & 04/01/17 & nd & 5.4 & nd & nd & nd & 0.20 & nd & nd & 0.17 & 0.068 \\
\hline TS-NWB-SS-W-111517 & $11 / 15 / 17$ & nd & 11. & 0.46 & 0.11 & 0.11 & 0.35 & nd & nd & nd & 0.11 \\
\hline \multicolumn{12}{|c|}{ Beaverdam Creek } \\
\hline TS-LBC-SS-W-012517 & $01 / 25 / 17$ & nd & 10 & nd & nd & nd & nd & nd & nd & 0.22 & 0.084 \\
\hline TS-LBC-SS-W-040117 & $04 / 01 / 17$ & nd & 14 & 2.2 & 1.5 & 2.0 & 0.39 & nd & nd & 0.17 & 0.17 \\
\hline TS-LBC-SS-W-040717 & $04 / 07 / 17$ & nd & 7.6 & nd & nd & nd & nd & nd & nd & nd & 0.21 \\
\hline TS-LBC-SS-W-052417 & $05 / 24 / 17$ & nd & 7.3 & nd & nd & nd & nd & nd & nd & nd & 0.15 \\
\hline TS-LBC-SS-W-110317 & $11 / 03 / 17$ & 0.37 & 23 & nd & nd & nd & nd & nd & nd & nd & 0.90 \\
\hline \multicolumn{12}{|c|}{ Watts Branch } \\
\hline TS-WB-SS-W-111317 & $11 / 13 / 17$ & nd & 22 & 1.8 & 1.9 & 1.6 & 0.60 & 0.73 & 0.17 & nd & nd \\
\hline TS-WB-SS-W-040117 & $04 / 01 / 17$ & nd & nd & 0.72 & 1.2 & 1.5 & 0.28 & nd & nd & nd & 0.11 \\
\hline TS-WB-SS-W-052417 & $05 / 24 / 17$ & nd & 20 & 1.7 & 2.6 & 4.7 & nd & nd & nd & nd & 0.31 \\
\hline \multicolumn{12}{|c|}{ Hickey Run } \\
\hline TS-HR-SS-W-012517 & $01 / 25 / 17$ & nd & 14 & 1.9 & nd & nd & 0.76 & nd & nd & 0.13 & 0.30 \\
\hline TS-HR-SS-W-040117 & $04 / 01 / 17$ & nd & 12 & 1.7 & 1.0 & nd & 0.52 & nd & nd & nd & 0.11 \\
\hline TS-HR-SS-W-052417 & $05 / 24 / 17$ & nd & 20 & 5.5 & 7.4 & nd & 0.89 & nd & nd & nd & 0.52 \\
\hline TS-HR-SS-W-111317 & $11 / 13 / 17$ & nd & 20 & 5.5 & 23 & nd & 0.54 & nd & 0.15 & nd & nd \\
\hline TS-HR-SS-W-111517 & $11 / 15 / 17$ & nd & 21 & 4.3 & 3.7 & nd & nd & 0.63 & nd & nd & nd \\
\hline \multicolumn{12}{|c|}{ Nash Run } \\
\hline TS-NR-SS-W-080117 & $08 / 01 / 17$ & nd & nd & 0.23 & nd & nd & 0.04 & nd & nd & nd & nd \\
\hline \multicolumn{12}{|c|}{ Pope Branch } \\
\hline TS-PB-SS-W-081417 & $08 / 14 / 17$ & nd & nd & 5.4 & 17 & 21 & nd & 0.97 & nd & nd & 0.28 \\
\hline \multicolumn{12}{|c|}{ Fort DuPont Creek } \\
\hline TS-FDP-SS-W-081417 & $08 / 14 / 17$ & nd & nd & 1.3 & 0.71 & 1.1 & nd & nd & nd & nd & nd \\
\hline \multicolumn{12}{|c|}{ Fort Stanton Creek } \\
\hline TS-FS-SS-W-101117 & $10 / 11 / 17$ & nd & nd & nd & nd & nd & nd & nd & nd & nd & nd \\
\hline
\end{tabular}


Table 13. Concentrations of metals in suspended-sediment samples from tributaries to the Anacostia River.

[Concentrations of metals in micrograms per kilogram; moisture content in percent by weight; sample date in parentheses]

\begin{tabular}{|c|c|c|c|c|c|c|c|c|}
\hline \multirow[b]{2}{*}{ Constituent } & \multicolumn{2}{|c|}{ Beaverdam Creek } & \multicolumn{3}{|c|}{ Watts Branch } & \multirow{2}{*}{$\begin{array}{c}\text { Hickey Run } \\
\text { TS-HR-LVSSD- } \\
\text { W-072817 } \\
\text { (7/28/17) }\end{array}$} & \multirow{2}{*}{$\begin{array}{c}\text { Pope Branch } \\
\text { TS-PB-LVSSD- } \\
\text { W-080717 } \\
\text { (8/7/17) }\end{array}$} & \multirow{2}{*}{$\begin{array}{c}\text { Fort DuPont Creek } \\
\text { TS-FDP-LVSSD- } \\
\text { W-080717 } \\
(8 / 7 / 17)\end{array}$} \\
\hline & $\begin{array}{c}\text { TS-LBC-LVSSD- } \\
\text { W-071817 } \\
(7 / 17 / 17)\end{array}$ & $\begin{array}{c}\text { TS-LBC-LVSSD- } \\
\text { W-072817 } \\
(7 / 28 / 17)\end{array}$ & $\begin{array}{c}\text { TS-WB-LVSSD- } \\
\text { W-071817 } \\
(7 / 17 / 17)\end{array}$ & $\begin{array}{c}\text { TS-WB-LVSSD- } \\
\text { W-072217 } \\
(7 / 22 / 17)\end{array}$ & $\begin{array}{c}\text { TS-WB-LVSSD- } \\
\text { W-072317 } \\
(7 / 23 / 17)\end{array}$ & & & \\
\hline Aluminum & 15,000 & 6,100 & 6,300 & 8,900 & 14,000 & 6,700 & 2,600 & 4,000 \\
\hline Antimony & 24 & 1.6 & 2.3 & 3.0 & 1.8 & 4.0 & 2.2 & 0.51 \\
\hline Arsenic & 15 & 7.8 & 7.6 & 9.8 & 14 & 4.5 & 6.2 & 4.6 \\
\hline Barium & 420 & 71 & 120 & 140 & 140 & 110 & 53 & 70 \\
\hline Beryllium & 1.0 & 1.1 & 1.2 & 1.4 & 1.6 & 0.48 & 0.2 & 0.77 \\
\hline Cadmium & 8.6 & 1.1 & 1.5 & 1.8 & 3.0 & 0.89 & 0.26 & 0.23 \\
\hline Calcium & 18,000 & 4,800 & 7,300 & 7,900 & 6,600 & 12,000 & 1,800 & 1,400 \\
\hline Chromium & 87 & 33 & 26 & 41 & 50 & 38 & 11 & 16 \\
\hline Cobalt & 29 & 29 & 34 & 59 & 35 & 9.9 & 6.7 & 18 \\
\hline Copper & 1,300 & 74 & 80 & 110 & 85 & 110 & 22 & 20 \\
\hline Iron & 48,000 & 28,000 & 25,000 & 35,000 & 45,000 & 17,000 & 29,000 & 20,000 \\
\hline Lead & 790 & 60 & 82 & 110 & 110 & 130 & 17 & 29 \\
\hline Magnesium & 8,900 & 2,200 & 3,400 & 4,100 & 3,400 & 5,600 & 800 & 690 \\
\hline Manganese & 650 & 500 & 660 & 1,300 & 750 & 240 & 1,500 & 470 \\
\hline Mercury & 2.2 & 0.085 & 0.18 & 0.24 & 0.13 & 0.14 & 0.031 & 0.11 \\
\hline Nickel & 100 & 38 & 44 & 63 & 55 & 34 & 6.7 & 14 \\
\hline Moisture content & 79 & 65 & 78 & 87 & 74 & 77 & 70 & 53 \\
\hline Potassium & 1,200 & 940 & 910 & 1,300 & 1,700 & 1,100 & 440 & 510 \\
\hline Selenium & 1.2 & 0.99 & 1.4 & 1.6 & 2.1 & 0.56 & 0.99 & 0.76 \\
\hline Silver & 3.9 & 0.2 & 0.54 & 0.39 & 0.31 & 0.5 & 0.11 & 0.52 \\
\hline Sodium & 420 & 91 & 220 & 280 & 130 & 270 & 170 & 57 \\
\hline Thallium & 0.22 & 0.17 & 0.2 & 0.21 & 0.38 & 0.11 & 0.054 & 0.1 \\
\hline Vanadium & 55 & 45 & 35 & 53 & 56 & 39 & 18 & 31 \\
\hline Zinc & 3,800 & 300 & 340 & 490 & 390 & 540 & 71 & 66 \\
\hline
\end{tabular}


Table 14. Concentrations of metals in bed sediment in tributaries to the Anacostia River.

[Concentrations of metals in micrograms per kilogram; moisture content in percent by weight; sample date in parentheses]

\begin{tabular}{|c|c|c|c|c|c|c|c|c|c|c|c|}
\hline \multirow[b]{2}{*}{ Constituent } & \multicolumn{3}{|c|}{ Northeast Branch } & \multicolumn{3}{|c|}{ Northwest Branch } & \multicolumn{5}{|c|}{ Beaverdam Creek } \\
\hline & $\begin{array}{c}\text { TS-NEB-SS- } \\
\text { W-012517 } \\
(1 / 25 / 17)\end{array}$ & $\begin{array}{c}\text { TS-NEB-SS- } \\
\text { W-052417 } \\
(5 / 24 / 17)\end{array}$ & $\begin{array}{c}\text { TS-NEB-SS- } \\
\text { W-110317 } \\
(11 / 3 / 17)\end{array}$ & $\begin{array}{c}\text { TS-NWB-SS- } \\
\text { W-012517 } \\
(1 / 25 / 17)\end{array}$ & $\begin{array}{c}\text { TS-NWB-SS- } \\
\text { W-040117 } \\
(4 / 1 / 17)\end{array}$ & $\begin{array}{c}\text { TS-NWB-SS- } \\
\text { W-111517 } \\
(11 / 15 / 17)\end{array}$ & $\begin{array}{c}\text { TS-LBC-SS- } \\
\text { W-012517 } \\
(1 / 25 / 17)\end{array}$ & $\begin{array}{c}\text { TS-LBC-SS- } \\
\text { W-040117 } \\
(4 / 1 / 17)\end{array}$ & $\begin{array}{c}\text { TS-LBC-SS- } \\
\text { W-040717 } \\
(4 / 7 / 17)\end{array}$ & $\begin{array}{c}\text { TS-LBC-SS- } \\
\text { W-052417 } \\
(5 / 24 / 17)\end{array}$ & $\begin{array}{c}\text { TS-LBC-SS- } \\
\text { W-110317 } \\
(11 / 3 / 17)\end{array}$ \\
\hline Aluminum & 620 & 760 & 980 & 1,600 & 1,900 & 2,600 & 1,100 & 1,200 & 1,300 & 790 & 1,600 \\
\hline Antimony & 0.07 & 0.036 & 0.14 & 0.038 & 0.06 & 0.088 & 0.18 & 0.2 & 0.17 & 0.15 & 0.42 \\
\hline Arsenic & 0.75 & 0.525 & 0.82 & 0.46 & 0.58 & 0.84 & 1.6 & 1.8 & 1.5 & 1.4 & 1.9 \\
\hline Barium & 7.1 & 6.5 & 11 & 8.1 & 11 & 23 & 11 & 13 & 12 & 7.0 & 24 \\
\hline Beryllium & 0.2 & 0.17 & 0.2 & 0.22 & 0.24 & 0.25 & 0.37 & 0.44 & 0.38 & 0.24 & 0.35 \\
\hline Cadmium & 0.04 & 0.039 & 0.064 & 0.027 & 0.045 & 0.077 & 0.14 & 0.13 & 0.17 & 0.13 & 0.24 \\
\hline Calcium & 4,600 & 2,200 & 3,800 & 670 & 680 & 1,000 & 560 & 1,800 & 650 & 420 & 1,100 \\
\hline Chromium & 6.6 & 4.4 & 6.9 & 6.3 & 14 & 13 & 7.5 & 8.1 & 7.9 & 6.6 & 12 \\
\hline Cobalt & 2.4 & 2.9 & 3.8 & 2.4 & 3.0 & 4.4 & 3.1 & 5.7 & 3.2 & 2.9 & 5.3 \\
\hline Copper & 4.0 & 2.9 & 7.4 & 7.6 & 5.4 & 11 & 37 & 8.5 & 7.9 & 5.7 & 18 \\
\hline Iron & 4,400 & 3,900 & 6,400 & 4,500 & 5,900 & 8,500 & 8,500 & 11,000 & 7,800 & 8,200 & 9,800 \\
\hline Lead & 2.7 & 3.35 & 6.1 & 3.3 & 4.8 & 20 & 16 & 35 & 12 & 10 & 22 \\
\hline Magnesium & 2,700 & 1,050 & 2,100 & 1,400 & 2,000 & 1,700 & 630 & 1,600 & 570 & 390 & 770 \\
\hline Manganese & 91 & 66 & 140 & 61 & 77 & 86 & 64 & 140 & 58 & 59 & 91 \\
\hline Mercury & 0.0078 & 0.01 & 0.0061 & 0.0045 & 0.0055 & 0.0055 & 0.0069 & 0.0086 & 0.0078 & 0.012 & 0.02 \\
\hline Nickel & 4.3 & 4.4 & 6.9 & 9.1 & 13 & 12 & 6.6 & 13 & 5.2 & 4.1 & 7.8 \\
\hline Moisture content & 19 & 24 & 40 & 22 & 26 & 34 & 23.7 & 21.5 & 22.7 & 22.8 & 35.3 \\
\hline Potassium & 73 & 140 & 180 & 330 & 420 & 720 & 380 & 230 & 510 & 320 & 570 \\
\hline Selenium & 0.069 & 0.1 & 0.16 & 0.072 & 0.08 & 0.34 & 0.093 & 0.1 & 0.12 & 0.092 & 0.31 \\
\hline Silver & 0.015 & 0.0089 & 0.027 & 0.008 & 0.0088 & 0.025 & 0.041 & 0.021 & 0.021 & 0.0087 & 0.077 \\
\hline Sodium & 44 & 33 & 41 & 50 & 57 & 55 & 97 & 58 & 85 & 36 & 54 \\
\hline Thallium & 0.013 & 0.017 & 0.021 & 0.035 & 0.037 & 0.071 & 0.016 & 0.024 & 0.02 & 0.013 & 0.032 \\
\hline Vanadium & 5.2 & 4.5 & 8.1 & 4.5 & 7.3 & 11 & 10 & 13 & 9.6 & 9.4 & 14 \\
\hline Zinc & 15 & 24 & 24 & 14 & 17 & 29 & 50 & 37 & 46 & 42 & 83 \\
\hline
\end{tabular}


Table 14. Concentrations of metals in bed sediment in tributaries to the Anacostia River.-Continued

[Concentrations of metals in micrograms per kilogram; moisture content in percent by weight; sample date in parentheses]

\begin{tabular}{|c|c|c|c|c|c|c|c|c|c|c|c|c|}
\hline \multirow[b]{2}{*}{ Constituent } & \multicolumn{3}{|c|}{ Watts Branch } & \multicolumn{5}{|c|}{ Hickey Run } & \multirow{2}{*}{$\begin{array}{c}\text { Nash Run } \\
\text { TS-NR-SS- } \\
\text { W-080117 } \\
\text { (8/1/17) }\end{array}$} & \multirow{2}{*}{$\begin{array}{c}\text { Pope Branch } \\
\text { TS-PB-SS- } \\
\text { W-081417 } \\
(8 / 14 / 17)\end{array}$} & \multirow{2}{*}{$\begin{array}{c}\begin{array}{c}\text { Fort DuPont } \\
\text { Creek }\end{array} \\
\text { TS-FDP-SS- } \\
\text { W-081417 } \\
\text { (8/14/17) } \\
\end{array}$} & \multirow{2}{*}{$\begin{array}{c}\begin{array}{c}\text { Fort Stanton } \\
\text { Creek }\end{array} \\
\text { TS-FS-SS- } \\
\text { W-101117 } \\
(10 / 11 / 17) \\
\end{array}$} \\
\hline & $\begin{array}{c}\text { TS-WB-SS- } \\
\text { W-111317 } \\
(11 / 13 / 17)\end{array}$ & $\begin{array}{c}\text { TS-WB-SS- } \\
\text { W-040117 } \\
(4 / 7 / 17)\end{array}$ & $\begin{array}{c}\text { TS-WB-SS- } \\
\text { W-052417 } \\
(5 / 24 / 17)\end{array}$ & $\begin{array}{c}\text { TS-HR-SS- } \\
\text { W-012517 } \\
(1 / 25 / 17) \\
\end{array}$ & $\begin{array}{c}\text { TS-HR-SS- } \\
\text { W-040117 } \\
(4 / 1 / 17) \\
\end{array}$ & $\begin{array}{c}\text { TS-HR-SS- } \\
\text { W-052417 } \\
(5 / 24 / 17) \\
\end{array}$ & $\begin{array}{l}\text { TS-HR-SS- } \\
\text { W-111317 } \\
(11 / 13 / 17) \\
\end{array}$ & $\begin{array}{l}\text { TS-HR-SS- } \\
\text { W-111517 } \\
(11 / 15 / 17) \\
\end{array}$ & & & & \\
\hline Aluminum & 1,500 & 1,100 & 1,200 & 2,600 & 3,800 & 2,600 & 2,700 & 1,600 & 980 & 1,500 & 800 & 1,700 \\
\hline Antimony & 0.33 & 0.13 & 0.16 & 1,100 & 0.46 & 0.49 & 0.61 & 0.5 & 0.11 & 0.086 & 0.069 & 0.23 \\
\hline Arsenic & 2.6 & 1.5 & 1.7 & 0.86 & 1.7 & 1.8 & 2.7 & 1.6 & 2.6 & 2.2 & 2.4 & 5.9 \\
\hline Barium & 18 & 15 & 41 & 3.5 & 54 & 49 & 41 & 32 & 11 & 9.8 & 8.2 & 19 \\
\hline Beryllium & 0.55 & 0.34 & 0.32 & 36 & 0.44 & 0.17 & 0.17 & 0.099 & 0.48 & 0.24 & 0.24 & 0.64 \\
\hline Cadmium & 0.18 & 0.11 & 0.19 & 0.42 & 0.31 & 0.145 & 0.15 & 0.14 & 0.06 & 0.1 & 0.026 & 0.19 \\
\hline Calcium & 2,600 & 1,200 & 1,500 & 0.11 & 27,000 & 23,000 & 28,000 & 27,000 & 330 & 2,200 & 250 & 480 \\
\hline Chromium & 8.7 & 9.0 & 8.7 & 40,000 & 16 & 41 & 25 & 16 & 6.8 & 21 & 9.5 & 24 \\
\hline Cobalt & 7.0 & 5.3 & 6.4 & 26 & 4.3 & 5.4 & 5.3 & 3.8 & 6.6 & 10 & 1.2 & 4.9 \\
\hline Copper & 11 & 7.4 & 15 & 6.6 & 25 & 27.5 & 210 & 27 & 5.6 & 7.8 & 2.5 & 10 \\
\hline Iron & 13,000 & 8,700 & 9,500 & 22 & 13,000 & 18,000 & 13,000 & 11,000 & 12,000 & 11,000 & 12,000 & 34,000 \\
\hline Lead & 24 & 12 & 150 & 24,000 & 76 & 88 & 43 & 16 & 7.3 & 15 & 3.1 & 8.9 \\
\hline Magnesium & 1,400 & 1,900 & 910 & 34 & 11,000 & 10,500 & 11,000 & 14,000 & 500 & 17,000 & 120 & 210 \\
\hline Manganese & 170 & 94 & 93 & 21,000 & 300 & 285 & 310 & 190 & 120 & 91 & 38 & 240 \\
\hline Mercury & 0.0079 & 0.0076 & 0.013 & 530 & 0.0068 & 0.0105 & 0.0094 & 0.0085 & 0.019 & 0.01 & 0.0099 & 0.0096 \\
\hline Nickel & 13 & 16 & 9.4 & 0.0068 & 17 & 35 & 29 & 27 & 8.9 & 210 & 1.5 & 6.0 \\
\hline Moisture content & 25.2 & 23.1 & 27.7 & 19 & 17 & 21.65 & 24.9 & 24.8 & 17.4 & 23.9 & 21.8 & 11.9 \\
\hline Potassium & 240 & 230 & 280 & 31 & 260 & 200 & 260 & 180 & 96 & 99 & 74 & 150 \\
\hline Selenium & 0.41 & 0.097 & 0.18 & 200 & 0.13 & 0.25 & 0.26 & 0.14 & 0.21 & 0.21 & 0.23 & 0.41 \\
\hline Silver & 0.14 & 0.026 & 0.0099 & 0.14 & 0.035 & 0.022 & 0.047 & 0.022 & 0.016 & 0.013 & 0.0084 & 0.011 \\
\hline Sodium & 48 & 54 & 51 & 0.029 & 290 & 225 & 230 & 150 & 33 & 41 & 21 & 26 \\
\hline Thallium & 0.026 & 0.026 & 0.035 & 200 & 0.047 & 0.033 & 0.041 & 0.028 & 0.025 & 0.024 & 0.011 & 0.024 \\
\hline Vanadium & 14 & 13 & 12 & 0.093 & 17 & 23 & 19 & 18 & 14 & 11 & 11 & 67 \\
\hline Zinc & 55 & 31 & 55 & 32 & 57 & 75 & 57 & 62 & 26 & 250 & 7.3 & 42 \\
\hline
\end{tabular}




\section{Sediment and Chemical Loads}

The loads of sediment and sediment-bound chemicals are defined as the mass of material transported by a stream over a given interval of time. Calculating loads involves combining three data types: the volume of water discharged $(\mathrm{Q})$, the concentration of suspended material in the water (SSC or POC), and the concentration of the sediment-bound chemical of interest. This section describes the methods and reviews the completeness and representativeness of the data types available to calculate sediment and contaminant loads.

\section{General Load Calculations}

As discussed by Runkel and others (2004), the method used to calculate sediment loads in a stream is based on the total mass moved over a selected time interval, and is calculated by using an integration of the load equation:

$$
L_{t}=\int_{0}^{t} Q C \mathrm{dt}
$$

where

$$
\begin{array}{ll}
C & \text { is concentration (mass/volume) } \\
L_{t} & \text { is the total load (mass/time), } \\
Q & \text { is instantaneous streamflow (volume/time) } \\
& \text { and } \\
t & \text { is time. }
\end{array}
$$

In this integral, $Q$ and $C$ are functions of time.

When the mass load of a chemical bound to material suspended in a stream is calculated, the load equation (1) is expanded to include the concentration of the chemical:

$$
L_{t}=\int_{0}^{t} Q C_{s s} C_{c} \mathrm{dt}
$$

where

$$
\begin{aligned}
& C_{s s} \quad \text { is the concentration of suspended materials } \\
& \text { (mass/liter of water), and } \\
& C_{c} \quad \text { is the concentration of the chemical of } \\
& \text { interest (mass/unit mass of sediment). }
\end{aligned}
$$

In this case, the chemical concentration is in units of mass per mass of sediment. Chemicals associated with suspended materials are contained within the physical structure of solids or sorbed onto the grain surfaces. In the case of hydrophobic chemicals such as PCBs/PAHs, the chemicals are commonly associated with organic carbon materials covering the sediment.

The principal source for discharge data is the USGS gaging stations; these stations typically measure discharge in units of cubic feet per second at short (5- to 15-minute) time intervals. Concentrations of suspended materials (SSCs) or chemical data are provided in units of mass per unit volume of water and mass of chemical per unit mass of sediment, respectively.

The continuous load integral equations (1) and (2) can be approximated by a discretized equation for total load:

$$
L_{t}=\sum_{i=1}^{N P} \Delta t_{i}\left(Q C_{s s}\right)_{i}=\sum_{i=1}^{N P} \Delta t_{i} L_{i}
$$

where

$L_{t} \quad$ is the total load over the time interval (mass/ time),

$L_{i} \quad$ is the instantaneous load (mass),

$N P \quad$ is the total number of measurements available,

$Q_{i} \quad$ is the discharge at time $i$ (volume/time),

$C_{s s, i}$ is the concentration (mass of sediment or $\mathrm{COC} /$ mass of sediment) at time $i$, and

$t_{i} \quad$ is the time step of interest (time),

This equation provides total load from the sum of the instantaneous loads measured at each time interval. When sediment-bound chemicals are of interest, the approximation equation is further expanded to give the total load of the chemical $\left(L_{T, C}\right)$ :

$$
L_{T, C}=\sum_{i=1}^{N P} \Delta t_{i} Q_{i}\left(C_{s s, i} \times C_{c, i}\right)_{i}
$$

This is the discretized equation for calculating sedimentbound chemical loads. Commonly, only a few sediment concentrations are available (especially for samples collected over small time steps); typically, even fewer measurements of sediment-bound chemical concentrations are available. Therefore, it is common to approximate this equation by removing the concentration of the chemical of interest:

$$
L_{T, C}=\hat{C}_{c, i} \sum_{i=1}^{N P} \Delta t_{i} \times Q_{i} \times C_{s s, i}
$$

where

$\hat{C}_{c, i} \quad$ is a representative concentration of the chemical of interest associated with the suspended sediment (mass/mass).

It is sometimes the case that $C_{s s, i}$ is moved outside the summation as a result of the lack of data points available. This concentration of the chemical of interest (or SSC) may be a single value or a statistical representation of multiple analyses, such as the average, median, or geometric mean.

The accuracy of a load calculation is a function of how well the individual component variables $\left(Q_{i}, C_{s s, i}, C_{c, i}\right)$ represent the actual values in the stream at each time step. The representativeness of a load calculation (how closely it approximates the true value) is, therefore, a function of (1) the uncertainty associated with each measurement of $Q, C_{s s}$, and 
$C_{c i}$; and (2) the size of the time step between measurements. Increasing the sampling rate (decreasing the time interval between measurements) helps capture the natural variation in the parameter, thereby producing a more "realistic" measure of the load.

A first-order-level estimate of the sediment (or COC) load is obtained by combining the average discharge (over the time of interest) and a single estimate of the suspendedsediment and COC concentrations. In this case, a single data value is used for each variable in the load equation. As an example, the sediment loads for the four major tributaries in this study were calculated by using the average annual discharge (measured at the USGS gaging stations) and an average of the suspended-sediment concentrations in samples collected during 2017. These loads are presented in table 15. The discharge is well represented temporally by the average $Q$, as this value is derived from measurements made at 5- or 15-minute intervals. SSC is less well represented by the average because of the limited number of SSC data available and the limited range of discharges they represent. For example, SSC in NEB is represented by 78 data points, only 21 percent of the possible daily values for 2017 . These SSC values are associated with samples collected over a narrow range of discharges, thereby skewing the average value. Using these values provides a total sediment load for 2017 of $3.09 \times 10^{7} \mathrm{~kg} / \mathrm{yr}$; as described farther on, this value is close to but lower than the average calculated in the current study and biases the percentage of sediment attributable to the individual tributaries.

This type of analysis has for many years been typical of the technique used to estimate sediment loads in streams.
The infrequent sampling for suspended sediment results in an average SSC that is likely not sufficiently robust to capture the actual variation in SSCs that occurs during a given year.

The approach taken in this study was to combine measurements of discharge, made at a high temporal measurement rate, with SSCs estimated from turbidity measured at or near the same measurement rate as discharge. The shortening of $\Delta \mathrm{t}$ provides a nearly "continuous" estimate of SSC (and $Q$ ) that helps capture the variation in both parameters during storms and low-flow conditions, thereby increasing the accuracy of the sediment loading estimate. The tradeoff of this method comes from the uncertainty introduced by using the statistical relation among turbidity, discharge, and SSC.

The third dataset needed is representative concentrations of sediment-bound COCs. Typically, it is cost prohibitive to analyze multiple samples from a single storm. To obtain a concentration, sediment was collected during four storm events representing different seasons, and during one to two low-flow periods. Sediment collected during low-flow conditions was included because of the possibility that sediment chemistry differs when water velocities are low, times when only the smallest grain-size particles are being transported. This scheme provided a dataset that, to the extent possible, captured the variations in $\mathrm{COC}$ concentrations.

The following sections describe the datasets used and presents loading estimates for sediment and sediment-bound COCs. The principal focus of this study is the annual loadings for 2017; loadings for the individual storms are provided in appendix 1 . Because continuous discharge and turbidity data were available, sediment loadings for NEB, Watts Branch, and Hickey Run were also calculated for 2013 through 2017.

Table 15. Estimated sediment loads for Northeast Branch, Northwest Branch, Watts Branch, and Hickey Run, 2017.

$\left[\mathrm{ft}^{3} / \mathrm{s}\right.$, cubic feet per second; L, liters; SSC, suspended-sediment concentration; min, minutes; $\mathrm{mg} / \mathrm{L}$, milligrams per liter; $\mathrm{kg}$, kilograms; --, not applicable]

\begin{tabular}{lccccccc}
\hline \multicolumn{1}{c}{ Tributary } & $\begin{array}{c}\text { Average } \\
\text { discharge } \\
\text { (ft } \mathbf{3} / \mathbf{s})\end{array}$ & $\begin{array}{c}\text { Time interval } \\
\text { of discharge } \\
\text { measurement } \\
\text { (min) }\end{array}$ & $\begin{array}{c}\text { Total } \\
\text { discharge } \\
(\mathbf{L})\end{array}$ & $\begin{array}{c}\text { Average } \\
\text { SSC }^{2} \\
\text { (mg/L) }\end{array}$ & $\begin{array}{c}\text { Number of } \\
\text { SSC data } \\
\text { points } \\
\text { available }\end{array}$ & $\begin{array}{c}\text { Total } \\
\text { sediment } \\
\text { load } \\
\text { (kg) }\end{array}$ & $\begin{array}{c}\text { Percentage } \\
\text { of total } \\
\text { predicted } \\
\text { sediment load }\end{array}$ \\
\hline Northeast Branch & 67.5 & 5 & $6.02 \times 10^{10}$ & 248 & 78 & $1.49 \times 10^{7}$ & 48 \\
Northwest Branch & 46.2 & 5 & $4.20 \times 10^{10}$ & 361 & 46 & $1.52 \times 10^{7}$ & 49 \\
Watts Branch & 2.80 & 2 & $2.42 \times 10^{9}$ & 172 & 81 & $4.16 \times 10^{5}$ & 1.3 \\
Hickey Run & 1.67 & 2 & $1.53 \times 10^{9}$ & 219 & 68 & $3.35 \times 10^{5}$ & 1.1 \\
Total & 118 & -- & $1.06 \times 10^{11}$ & 250 & 273 & $3.09 \times 10^{7}$ & 100 \\
\hline
\end{tabular}

\footnotetext{
${ }^{1}$ Average discharge reported by U.S. Geological Survey corrected to calendar year 2017.

${ }^{2}$ Average SSC, in mg/L, for samples collected by U.S. Geological Survey in calendar year 2017.
} 


\section{Discharge in Gaged Tributaries}

Discharge data were obtained from the USGS National Water Information System (NWIS) database dataset (U.S. Geological Survey, 2019), where discharge data are derived from stage by using relations that have been established, in some cases, over many years. The stage-discharge relations for the streams are confirmed yearly and, if necessary, corrected for changes (temporary or long-term) in the conditions of the channels and basins. For the NEB and NWB, discharge measurements are available beginning in 1939, whereas discharge in Watts Branch and Hickey Run are available from 2012. Discharge measurements for LBDC began with this study in late 2016. Inspection of available discharge data (except those for LBDC) shows that 91 to 100 percent of the possible measurements made at 5- or 15-minute intervals were available for 2013-17. During 2017, 98 percent (NEB) to 100 percent (NWB) of the possible measurements were available; 93 percent of the possible measurements were available for LBDC. After tabulating the discharge values, any missing data were identified and, when possible, replaced with estimated values. Missing data represent times when equipment was not working or was being repaired, or when ice was present in the streams. Fortunately, few gaps existed in the discharge record for the gaged tributaries, typically representing less than 5 percent of the yearly record. Approximately 25 percent of the LBDC record contained discharge measurements recorded at 1- or 2-hour intervals; these intervals were filled by using the interpolation methods described below. Even when these gaps are considered, little bias was expected as a result of replacing missing values with estimated data.

In making long-time load calculations, procedures used to replace missing data must be documented and applied consistently, as loads can vary with the procedures used to estimate missing data. The following methods were used to replace missing discharge data in this study:

1. For periods of missing data lasting less than 60 minutes, values were estimated by averaging the discharge measured at the beginning and end times of each interval.

2. For missing data intervals lasting from 60 minutes to 3 days, values were estimated from the gage height (when available) and the stream stage-discharge relation. When gage height was not available, the time interval in an adjoining stream and the precipitation record were inspected to determine whether a storm event had occurred. If a storm was not evident, then the missing data were replaced with the average of the discharge at the beginning and end of the gap. The record for LBDC contained several periods where data were reported at 1- or 2-hour intervals. These are average values for the intervals determined by the USGS technician approving the data.

3. Discharge at NEB and NWB was measured at 15-minute intervals, whereas turbidity was measured at 5-and, in some cases, 2-minute intervals. To obtain a 1:1 match with the turbidity data, the discharge at intervals between the 15-minute discharges was repeated until the next recorded measurement. For example, the discharge measured at 12:00 was entered for 12:05 and 12:10 to synchronize the discharge measurements with the turbidity measurements made at these times. The approach used to estimate missing turbidity measurements is discussed farther on in the report.

4. Because there is some upstream flow of water in LBDC, the discharge at $\mathrm{LBDC}$ required additional manipulation before it could be used in calculating sediment loads. Negative discharge was included for calculating total volume of water passing the gage, as any water moving upstream past the gage would undoubtedly move downstream in the future. However, at least three factors affect the accuracy of using turbidity as a surrogate for suspended sediment. First, the relation between turbidity and discharge used to estimate SSC was developed by using measurements and samples obtained only when flow was downstream, and thus were not suitable for upstream flow. Second, water velocities during upstream flow were typically very low, and therefore little energy was available for transporting sediment. Finally, the concrete structure located under the Route 50 bridge acts as an impediment to upstream transport of sediment. Therefore, when calculating sediment loads, negative discharge values were replaced by a null value $\left(0 \mathrm{ft}^{3} / \mathrm{s}\right)$ and thus did not contribute to the tally of the total yearly sediment load. Fortunately, upstream flow in LBDC occurred only over a relatively small percentage (6.8 percent [596 hours]) of the year.

Discharge in LBDC had not been measured prior to the installation of a gaging station as part of this study. LBDC is affected by tidal fluctuations in the level of the Anacostia River, which makes measurement of discharge difficult in the streams where gages are above the head of tide. Tidal cycling in the mainstem of the Potomac River and in the Anacostia River cause water in LBDC to "back up" as high tide approaches. With the approach of high tide, water is stored upstream from the mouth of the LBDC and the concrete structure under the Route 295 bridge. This storage is manifested by the rise in stage and the slowing of water velocity as high tide in the Anacostia River is approached. Water levels in the LBDC during nonstorm conditions typically varied daily between 1 and 2 Fort The gage-height data collected in this study show that at the point of high tide, water in LBDC may slowly flow upstream for several minutes; however, the concrete structure controls the farthest upstream point affected by the tide. Discharge and water levels during storms differ considerably from those during dry-weather conditions. Stormwater runoff into LBDC causes a rapid rise in water level, commonly beginning within 30 minutes of the onset of precipitation. Depending on the intensity of rainfall in the basin, peaks of stage more than $5 \mathrm{ft}$ have been observed to 
occur within 1 hour of onset of precipitation. Storm discharge leaving LBDC is affected by the stage in the Anacostia River, which is ultimately affected by discharge from NEB and NWB and the stage in the Potomac River.

The LBDC gaging station (USGS station 01651730) uses an acoustic Doppler velocity meter (ADVM) to determine water velocity and discharge. The ADVM was located at the Anacostia Sewage Treatment Plant, immediately downstream from a concrete structure beneath the Route 50 bridge (fig. 1B). ADVMs measure stage and stream velocity; these parameters are multiplied by the cross-channel area to yield discharge estimates. Calibration is required to establish the relation among stage, velocity, channel cross-sectional area, and discharge. The methods used to calibrate these values are described by Oberg and others (2005), Turnipseed and Sauer (2010), and Levesque and Oberg (2012). Calibration was conducted by making multiple cross-channel measurements of discharge by using a downward-facing acoustic Doppler current profiler (ADCP) that was rafted across the channel at different stages. The ADCP uses sound to measure water velocity in known layers (volumes) beneath the raft; these layers of discharge are summed to determine total discharge across the channel. The velocity and stage measured by the ADVM are then correlated to the total discharge to establish an index relation for the station. Checks of the index relation were made repeatedly throughout the year to correct for changes in channel morphology. Once a sufficient number of cross-channel discharge measurements are available, discharge is calculated by using the water velocity and stage measured by the ADVM.

A total of 157 cross-sectional measurements of discharge were made during low-flow and stormflow conditions during 2016-17 to relate water velocity and stage to discharge at the gaging station. The calibration measurements covered a range in stage from 0.05 to $4.48 \mathrm{ft}$ and a range in mean cross-channel velocity from -0.04 to 2.95 feet per second ( $\mathrm{ft} / \mathrm{s}$ ). (Negative values represent upstream flow.) Ultimately, the calibration dataset covered a range from 1.75 to 761 cubic feet per second $\left(\mathrm{ft}^{3} / \mathrm{s}\right)$. From these data, a two-variable model was developed to relate flow velocity and river stage to discharge. (Stage measurements are required to determine cross-sectional area of the channel).

Figure 4 shows the stage and velocity in LBDC measured during the March 31, 2017, storm event, when 0.935 in. of rain fell over 24 hours (with most of the 0.71 in. of rain falling between 10:50 and 16:50). The daily tidal cycle of stage and velocity, as well as the decrease in velocity as high and low tides were approached, prior to the storm are apparent. As high tide was approached, water briefly flowed upstream. Because this phenomenon was extremely brief, it is unlikely that sediment and chemicals were transported from the Anacostia River

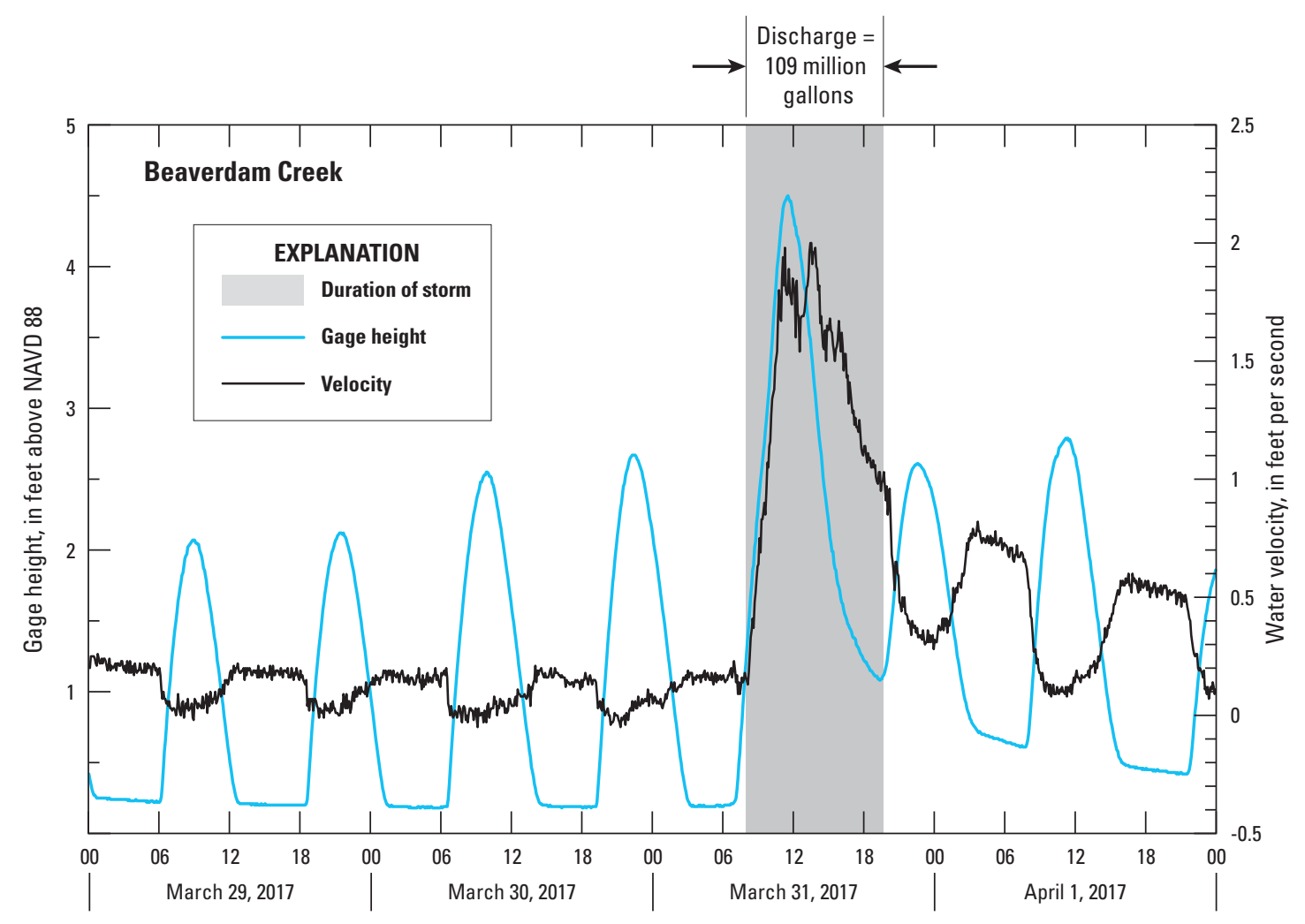

Figure 4. Stage and water velocity in Beaverdam Creek, March 15-April 1, 2017. (Gray shading represents duration of storm; NAVD 88, North American Vertical Datum of 1988) 
into LBDC. After precipitation began, the flow of stormwater was superimposed on the normal tidal stage and velocity cycles. Stage rose rapidly from $0.19 \mathrm{ft}$ at 5:20 to a maximum of $4.5 \mathrm{ft}$ at 11:30, when water velocity reached a peak of about $2 \mathrm{ft} / \mathrm{s}$. During the receding limb of the hydrograph, the lowest intertidal stage and water velocities remain elevated over prestorm values for at least the next three tide cycles.

\section{Discharge in Ungaged Tributaries}

Measuring discharge during storms in flashy, small urban streams with extreme flow velocities is difficult; flow ranges from a trickle (or even zero) to raging currents. Flow in these small tributaries begins almost instantly with the onset of rain and can peak and begin decreasing within minutes to a few hours after precipitation ends. Because deploying continuous discharge-measuring equipment on these streams is cost prohibitive, several attempts were made to measure discharge during storms. However, the short duration of stormflow and the hazardous wading conditions made these attempts unsuccessful. In all, only 16 measurements of discharge in the ungaged tributaries were made in this and in previous studies (table 16).

Discharge in Fort DuPont Creek was measured during a USGS study in 2000 (U.S. Geological Survey, 2000), when seven storm events were measured from June to December 1999. The stage variation was small, from 1 to $2 \mathrm{ft}$, representing discharges up to $70 \mathrm{ft}^{3} / \mathrm{s}$, and stormflow lasted only a few hours. A relation developed from the 1999 data predicts a large increase in discharge with a small rise in stage in these streams $(\mathrm{Q}=0.0475 \times \exp [3.4472 \times$ stage $])$; this relation predicts a 1 -ft rise in stage will result in about a five-fold increase in discharge.

Discharge during the dry summer months was reported to range from 0 to $0.125 \mathrm{ft}^{3} / \mathrm{s}$, but the difficulty in measuring discharge during low flow makes assigning a long-term base-flow discharge value difficult. Low flow ranges from $0.01 \mathrm{ft}^{3} / \mathrm{s}$ (the smallest value that can be measured with standard USGS field

Table 16. Discharge and stream measurements for Nash Run, Pope Branch, Fort DuPont Creek, and Fort Stanton Creek.

[ft, feet; $\mathrm{ft}^{3} / \mathrm{s}$, cubic feet per second; nr, not reported; <, less than; USGS, U.S. Geological Survey; DOEE, Washington, D.C., Department of Energy \& Environment]

\begin{tabular}{|c|c|c|c|c|c|}
\hline Stream & $\begin{array}{l}\text { Data } \\
\text { source }\end{array}$ & $\begin{array}{c}\text { Date of } \\
\text { measurement }\end{array}$ & $\begin{array}{c}\text { Stream } \\
\text { width } \\
\text { (ft) }\end{array}$ & $\begin{array}{c}\text { Mean } \\
\text { depth } \\
\text { (ft) }\end{array}$ & $\begin{array}{c}\text { Discharge } \\
\left(\mathrm{ft}^{3} / \mathrm{s}\right)\end{array}$ \\
\hline \multirow[t]{3}{*}{ Nash Run } & USGS $^{1}$ & $7 / 27 / 17$ & 7.0 & 0.3 & 1.0 \\
\hline & USGS & $4 / 27 / 18$ & 6.7 & 0.41 & 1.97 \\
\hline & & $1 / 12 / 01$ & $\mathrm{nr}$ & $\mathrm{nr}$ & 0.24 \\
\hline \multirow[t]{2}{*}{ Pope Branch } & USGS & $4 / 27 / 18$ & 5.7 & 0.74 & 0.80 \\
\hline & $\mathrm{DOEE}^{2}, 2012$ & $\mathrm{nr}$ & $\mathrm{nr}$ & $\mathrm{nr}$ & 0.24 \\
\hline \multirow[t]{10}{*}{ Fort DuPont Creek } & DOEE, 2012 & $6 / 4 / 99$ & $\mathrm{nr}$ & 2 & 47 \\
\hline & DOEE, 2012 & $6 / 29 / 99$ & $\mathrm{nr}$ & 1.2 & ${ }^{3} 3.0$ \\
\hline & DOEE, 2012 & $8 / 25 / 99$ & $\mathrm{nr}$ & $1.4-1.7$ & ${ }^{3} 6$ \\
\hline & DOEE, 2012 & 9/16/99 & $\mathrm{nr}$ & 1.6 & ${ }^{3} 12$ \\
\hline & DOEE, 2012 & $10 / 20 / 99$ & $\mathrm{nr}$ & 1.4 & 35 \\
\hline & DOEE, 2012 & $11 / 20 / 99$ & $\mathrm{nr}$ & 1.3 & ${ }^{3} 4$ \\
\hline & DOEE, 2012 & $12 / 10 / 99$ & $\mathrm{nr}$ & 1.5 & ${ }^{3} 8$ \\
\hline & USGS & $9 / 15 / 17$ & 20.5 & 0.1 & $<0.1$ \\
\hline & USGS & $4 / 27 / 18$ & 5.7 & 0.39 & 0.78 \\
\hline & DOEE, 2012 & $\mathrm{nr}$ & $\mathrm{nr}$ & $\mathrm{nr}$ & 0.7 \\
\hline \multirow[t]{2}{*}{ Fort Stanton Creek } & USGS & $4 / 27 / 18$ & 3.2 & 0.18 & 0.50 \\
\hline & DOEE, 2012 & $\mathrm{nr}$ & $\mathrm{nr}$ & $\mathrm{nr}$ & 0.05 \\
\hline
\end{tabular}

${ }^{1}$ Data available from U.S. Geological Survey National Water Information System database (U.S. Geological Survey, 2019).

${ }^{2}$ Data available from Washington, D.C., Department of Energy \& Environment, 2012.

${ }^{3}$ Discharge calculated from stage and relation $\mathrm{Q}=0.0475 \times \exp (3.4472 \times$ stage $)$, from Washington, D.C., Department of Energy \& Environment (2012). 
equipment) to roughly $2.0 \mathrm{ft}^{3} / \mathrm{s}$. Fort DuPont Creek and Fort Stanton Creek were observed to be dry for most of the summer of 2017, except when localized thunderstorms occurred in the area. This information indicates that peak stormflow in these streams is about 5 to $100 \mathrm{ft}^{3} / \mathrm{s}$.

Because of the difficulties encountered in measuring discharge, yearly discharge of water and sediment loadings in the ungaged tributaries were estimated from the Watts Branch Basin results by using the basin-area ratio method. This approach is justified because the land-use characteristics (urbanized watersheds) of the small basins and Watts Branch Basin are similar, and because the response of stage in the ungaged streams was shown to be nearly identical to the response in Watts Branch Basin. The basins are highly urbanized and are characterized by a large percentage of impervious surfaces and extensive road networks. Some differences between these basins and Watts Branch Basin do exist, however. Because their basins include park areas, the basins of Fort DuPont Creek and Fort Stanton Creek have impervious surface coverages of 12 and 11 percent, respectively (Washington, D.C., Department of Energy \& Environment, 2012), whereas impervious surface cover in Watts Branch Basin is 31 percent. However, these park areas are surrounded by residential areas and an extensive network of roads and storm sewers that route most of the precipitation falling on the basin directly to these streams. Storm drains were observed at nearly all road crossings, and stage in these tributaries was commonly observed to rise quickly with the onset of precipitation.

The second observation is the similarity in response to rainfall in the small tributaries and Watts Branch. Selfrecording pressure transducers were placed in the beds of several of the ungaged streams, allowing a comparison to be made between the response in stage to that in Watts Branch. Stage in Nash Run and Watts Branch during the rain event on July 18, 2017, when 0.25 in. of precipitation was recorded at the USGS rain gage at Hickey Run, is shown in figure 5. (The timing, duration, and amount of precipitation at Hickey Run was nearly identical to that measured at Ronald Reagan Washington National Airport). Prior to the onset of precipitation, stage and discharge in Nash Run and Watts Branch were low $\left(<0.1\right.$ and $0.56 \mathrm{ft}^{3} / \mathrm{s}$, respectively). Stage began to rise almost immediately with the onset of precipitation, and peak streamflow in the two streams occurred nearly simultaneously. Stage in Nash Run peaked at about $3.5 \mathrm{ft}$, whereas that in Watts Branch peaked at about 2.5 Fort Flow in Watts Branch receded slightly more slowly than flow in Nash Run, taking about 500 minutes to return to its prestorm level, as would be expected as a result of its larger basin area. Total discharge in Watts Branch was 6.19 Mgal $\left(2.35 \times 10^{7} \mathrm{~L}\right)$. If discharge in Nash Run is assumed to have averaged $10 \mathrm{ft}^{3} / \mathrm{s}$ for the 135 -minute

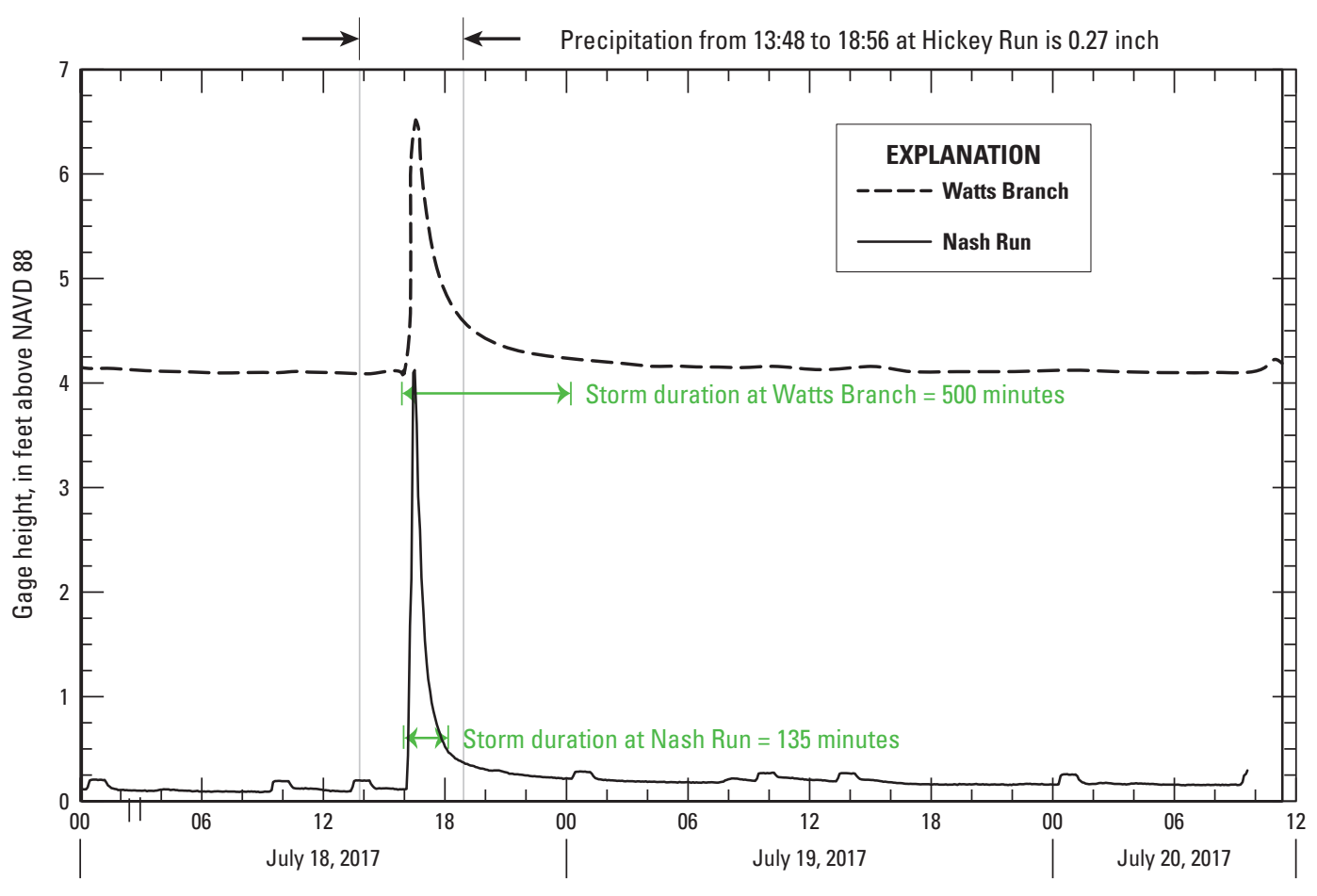

Figure 5. Water levels in Nash Run and Watts Branch, July 18-19, 2017. (NAVD 88, North American Vertical Datum of 1988) 
duration of the storm hydrograph and $1 \mathrm{ft}^{3} / \mathrm{s}$ for the remainder of the day, total discharge in Nash Run is estimated to be 1.2 Mgal. The fact that the ratio of total discharge in Nash Run to that in Watts Branch (0.19) is very close to the ratio of their basin areas (0.21) supports the use of the basin-area ratio method to establish annual discharge for the small, ungaged tributaries to the Anacostia River.

The basin areas of the ungaged tributaries normalized to that of the Watts Branch Basin and the estimated annual discharge for 2013-17 are listed in table 17. For 2017, the annual discharge from the ungaged tributaries totaled $420 \mathrm{Mgal}$.

Although the basin-area ratio method appears to be appropriate for determining discharge in these ungaged streams:

1. Pope Branch, Fort DuPont Creek, and Fort Stanton Creek all enter underground pipes immediately downstream from the sampling point in this study. Therefore, the sampling location where sediment was collected does not receive the runoff from a substantial portion of each basin. Many storm drains are likely connected to the underground conduit, especially storm drains from Route 205 . These drains presumably supply additional water, sediment, and chemicals to the Anacostia River that are not accounted for in this study. Using the basin-area ratio approach would account for this "extra" water, but not necessarily the sediment and chemicals it contains. Additional sampling at the point where storm sewers enter the Anacostia River is needed to characterize this additional input.

2. The total discharge for the ungaged basins has not been adjusted for the proportion of impervious cover. Therefore, in these calculations it is assumed that all precipitation falling on the basin is routed to the tributaries. Although some water undoubtedly is lost to evapotranspiration and infiltration, both losses would likely be small as a result of the presence of highly compacted soils and nonforested urban areas. The estimates in table 17, therefore, represent the maximum contribution of water, sediment, and contaminants expected from each tributary. As demonstrated farther on in this report, however, even these maximum contributions are only a small percentage of the discharge from the gaged tributaries.

\section{Estimating Suspended-Sediment Concentrations from Measurements of Continuous Turbidity}

Sediment loads are necessary to calculate sedimentbound chemical loadings. Therefore, it is essential to have accurate information about the amount of suspended sediment in each tributary. In this study, SSC was estimated from the turbidity measured by using water-quality sondes deployed at the sampling point in each tributary. Sondes were deployed either as part of the long-term water-quality monitoring being conducted by the USGS at NEB, NWB, LBDC, Watts Branch, and Hickey Run, or, in the case of the ungaged tributaries, in preparation for an upcoming storm event. To use turbidity as a surrogate for SSC, the relation between these two properties must first be established. This relation was developed by using simple and multiple linear regression methods following the procedures of Rasmussen and others (2011), which are based on the statistical methods described by Cohn and others (1989) and Helsel and Hirsch (2002). Many studies have demonstrated the use of turbidity as a surrogate measure of SSC (see, for example, Jastram and others, 2009; Wood and Teasdale, 2013; Miller and others, 2007).

Simple linear regression (SLR) and multiple linear regression (MLR) analysis were used to predict SSC from turbidity; the MLR relations were developed by using turbidity and discharge. Discharge was included because during

Table 17. Relative basin areas and estimated discharge for ungaged tributaries, 2013-17.

[mi², square miles; Mgal/yr, million gallons per year; --, not applicable]

\begin{tabular}{|c|c|c|c|c|c|c|c|c|}
\hline \multirow{2}{*}{ Tributary } & \multirow{2}{*}{$\begin{array}{c}\text { Basin area } \\
\left(\mathrm{mi}^{2}\right)\end{array}$} & \multirow{2}{*}{$\begin{array}{l}\text { Percent } \\
\text { impervious } \\
\text { cover }\end{array}$} & \multirow{2}{*}{$\begin{array}{l}\text { Relative } \\
\text { area }^{1}\end{array}$} & \multicolumn{5}{|c|}{$\begin{array}{c}\text { Estimated discharge } \\
\text { (Mgal/yr) }\end{array}$} \\
\hline & & & & 2017 & 2016 & 2015 & 2014 & 2013 \\
\hline Watts Branch & 3.36 & 31 & 1 & 674 & 757 & 960 & 1,090 & 1,040 \\
\hline Nash Run & 0.71 & 31 & 0.21 & 142 & 159 & 202 & 229 & 218 \\
\hline Pope Branch & 0.37 & 32 & 0.11 & 74 & 83 & 106 & 120 & 114 \\
\hline Fort DuPont Creek & 0.68 & 11 & 0.20 & 135 & 151 & 192 & 218 & 208 \\
\hline Fort Stanton Creek & 0.33 & 10 & 0.10 & 67 & 76 & 96 & 109 & 104 \\
\hline Total for ungaged streams & 2.09 & -- & -- & 420 & 470 & 600 & 680 & 640 \\
\hline
\end{tabular}

${ }^{1}$ Relative area is basin area divided by Watts Branch Basin area. 
high stormflow, water velocities are sufficient to entrain and transport sand-sized particles that may not affect turbidity in the stream. Sand-sized materials are ubiquitous in the unconsolidated surface materials in the Coastal Plain and are present in the "grit" washed off road surfaces into storm drains. Turbidity sondes do not respond to sand-sized materials in the same manner as they do to silt and clay (Sutherland and others, 2000). At the highest stormflow velocities, when sand is entrained in suspension, measurements of turbidity likely underestimate the suspended sediment load. Adding a term that relates Q and SSC helps to correct this underestimation.

Turbidity and discharge data available for regression analysis in this study are summarized in table 18 . Turbidity and SSC (in discrete grab samples) have been measured continuously at the NEB, Watts Branch, and Hickey Run gaging stations since 2013. Turbidity at NWB has been measured only since August 2015 and, as mentioned previously, turbidity at LBDC has been measured only since late December 2016. Measurements of turbidity and SSC made by the USGS

Table 18. Summary of available discharge, turbidity, and suspended-sediment concentration data for the Anacostia River tributaries.

[ft $3 / \mathrm{s}$, cubic feet per second; FNU, Formazin Nephelometric Units]

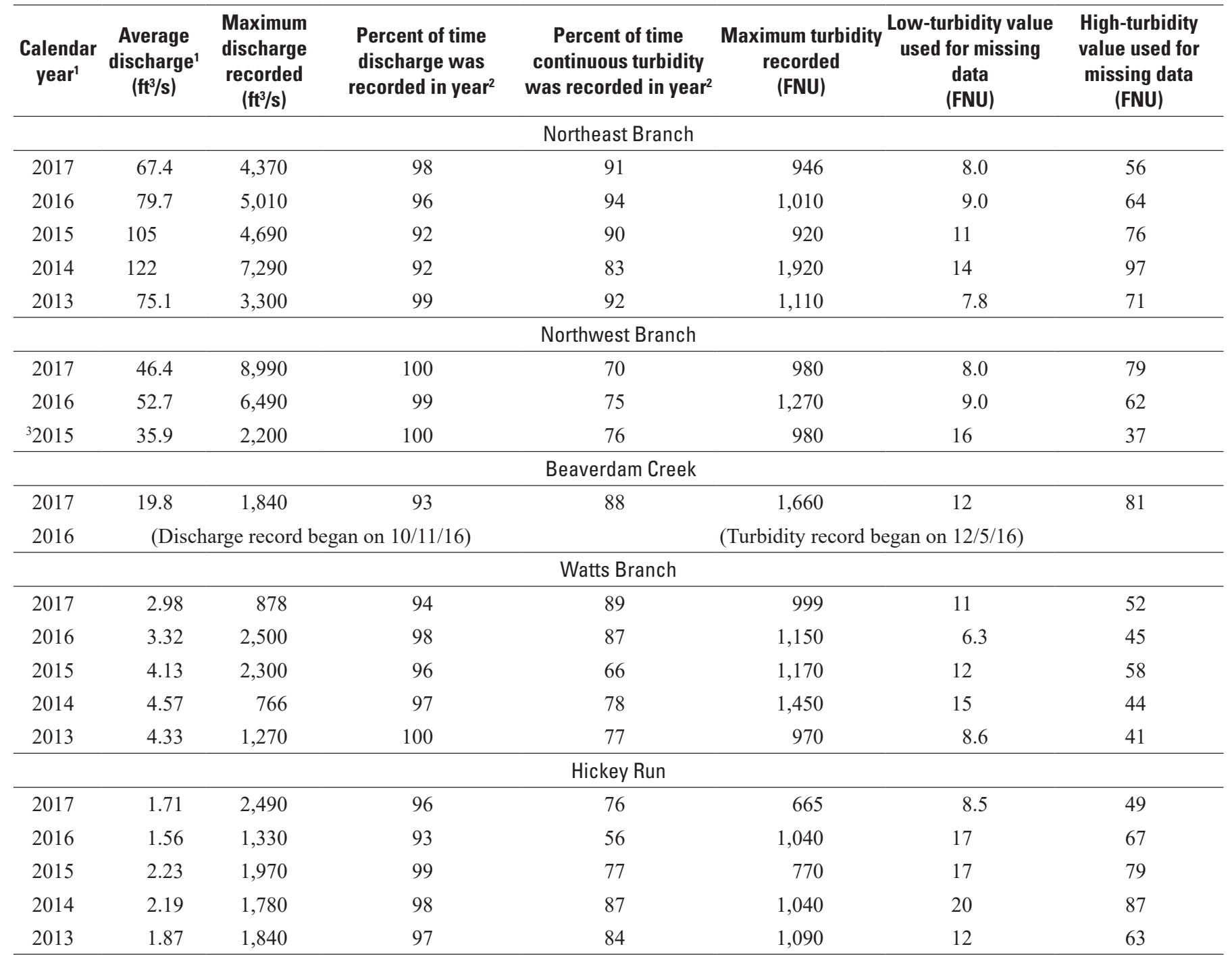

${ }^{1}$ Average discharge values were calculated for the calendar years indicated, January 1 through December 31 . They may not equal the average for water years, which extend from October 1 through September 31.

${ }^{2}$ Percentage of time covered is calculated as total number of measurements divided by the total possible measurements. For the 5-minute data intervals, the total number possible per year is 105,120 ; for 2-minute intervals, the total number is 262,800 measurements. The values listed here represent the dataset prior to estimation of missing values.

${ }^{3}$ Measurements on Northwest Branch began in August 2015. 
included conducting monthly sonde calibrations and cleaning (Wagner and others, 2006); these measurements were made by using the same methods used in the present study. Therefore, minimal method bias is expected to affect the comparison between the data collected before 2016 and the data collected in the present study.

Miller and others $(2007,2013)$ made continuous measurements of turbidity and specific conductance along with collecting samples for determination of SSCs in the NEB and NWB of the Anacostia River. They examined the cross-sectional variability in turbidity and specific conductance in the NEB and NWB to determine where to locate the autosampler intakes. Cross-channel turbidity and specific conductance were compared in samples collected on an equal-width basis and samples collected concurrently with automatic samplers. That work indicated that the locations of the intake nozzles of the autosampling equipment adequately represented the crosschannel composition of each stream. This present study used the same locations for sampling-line inlets.

Similarly, standardized USGS procedures have been used since 2003 to measure turbidity and collect samples for determination of suspended-sediment concentrations at Watts Branch and Hickey Run. Cross-channel measurements and suspended sediment sampling demonstrate that these streams are well mixed, and that the locations of the turbidity sonde and inlet lines represent the cross-channel variability in these streams. The nozzle of the intake line for the autosampler and turbidity sensor were set immediately adjacent to one another in each stream.

The location of the inlet nozzle and turbidity sensor at LBDC was dictated by the stream characteristics and the site conditions (including buried debris) and by the location of the streamflow-gaging equipment. The concrete structure under the Route 50 bridge, immediately upstream from the sampling location, likely caused substantial mixing in the stream. Data collected during the calibration of the gaging equipment helps confirm that the stream is indeed well mixed, and that turbidity measurements and suspended-sediment samples collected in this stream are representative of the conditions in the tributaries.

The inlet-line nozzle was placed mid-channel approximately $50 \mathrm{ft}$ downstream from the USGS gaging equipment. As described earlier, several cross-sectional discharge and velocity measurements were made at LBDC by using a downward-facing ADCP pulled across the channel. One of the cross-sectional profiles of velocity is shown in figure $6 \mathrm{~A}$. The ADCP measures discharge below the instrument in multiple (six to eight) layers of set thickness ( $1 \mathrm{ft}$ ); each colored block in this plot represents the water velocity within an indicated volume of water. This plot shows the water velocity was well distributed across the channel. Velocity near the left bank was about $1.9 \mathrm{ft} / \mathrm{s}$ greater than the velocity near the right bank; this velocity is sufficient to suspend clay, silt, and fine sand. Across the remainder of the channel, velocities were high and consistent $(6-7 \mathrm{ft} / \mathrm{s})$. Turbidity was also measured during the calibration effort by suspending a water-quality sonde from the ADCP raFort A transect of turbidity and specific conductance is presented in figure $6 \mathrm{~B}$. Turbidity was constant across the channel except for a peak approximately $15 \mathrm{ft}$ from the left bank. This peak likely is associated with an eddy formed downstream from the streamgaging equipment and shows an increase of about 30 percent above the average cross-channel turbidity (126 FNU). Because the intake nozzle was set farther into the middle of the channel, this spike is not considered problematic. On the basis of the data collected during calibration measurements, the LBDC channel appears well mixed and the discrete samples are considered to be representative of variability across the channel.

In the same manner as that used to evaluate discharge data, the turbidity datasets were retrieved from NWIS, tabulated, and inspected for completeness. Where data were missing, estimated values were substituted whenever possible. As mentioned earlier, turbidity and discharge were measured at 5-minute intervals at Watts Branch and Hickey Run, whereas at NEB and NWB, these measurements were made at 15- and 5-minute intervals, respectively. As mentioned previously, the discharge data for NEB and NWB were interpolated to produce a 1:1 relation with turbidity.

Turbidity measurements made by the USGS are reviewed at the end of each water year (September 31). Until they are reviewed, sonde data are flagged as provisional ("P"). Data review includes inspection by trained field technicians who apply shifts and corrections based on the monthly calibration checks made throughout the year, and removal of suspect data points. The corrected provisional data are then reviewed by a hydrologist with special proficiency in water quality. If the data are accepted, they are flagged as approved ("A").

The turbidity record, unlike the discharge record, contained many intervals of missing data. Data completeness over the period 2013-17, defined as the ratio of the number of measurements reported to the total number of measurements possible, multiplied by 100 , ranged from a low of 56 percent to a high of 94 percent. For 2017, the turbidity data were 91 percent complete for NEB, 70 percent complete for NWB, 88 percent complete for LBDC, 89 percent complete for Watts Branch, and 76 percent complete for Hickey Run. Missing turbidity data were the result of equipment failure or clogging, periods of equipment maintenance, or the presence of ice. Turbidity sensors, unlike the pressure systems used to measure stage, are vulnerable to fouling by algae, oil, and debris. To make the record as complete as possible, missing turbidity data were replaced in the following manner:

1. For data gaps lasting less than 120 minutes, missing turbidity values were estimated by using the average of the measurements at the beginning and end of the gap.

2. For data gaps lasting from 120 minutes to 3 days, missing turbidity data were estimated by using the average turbidity value for the year based on discharge. The average of the turbidity measured when $\mathrm{Q}$ was below the mean annual discharge (low-flow turbidity) and the average turbidity measured when Q exceeded the mean 
A

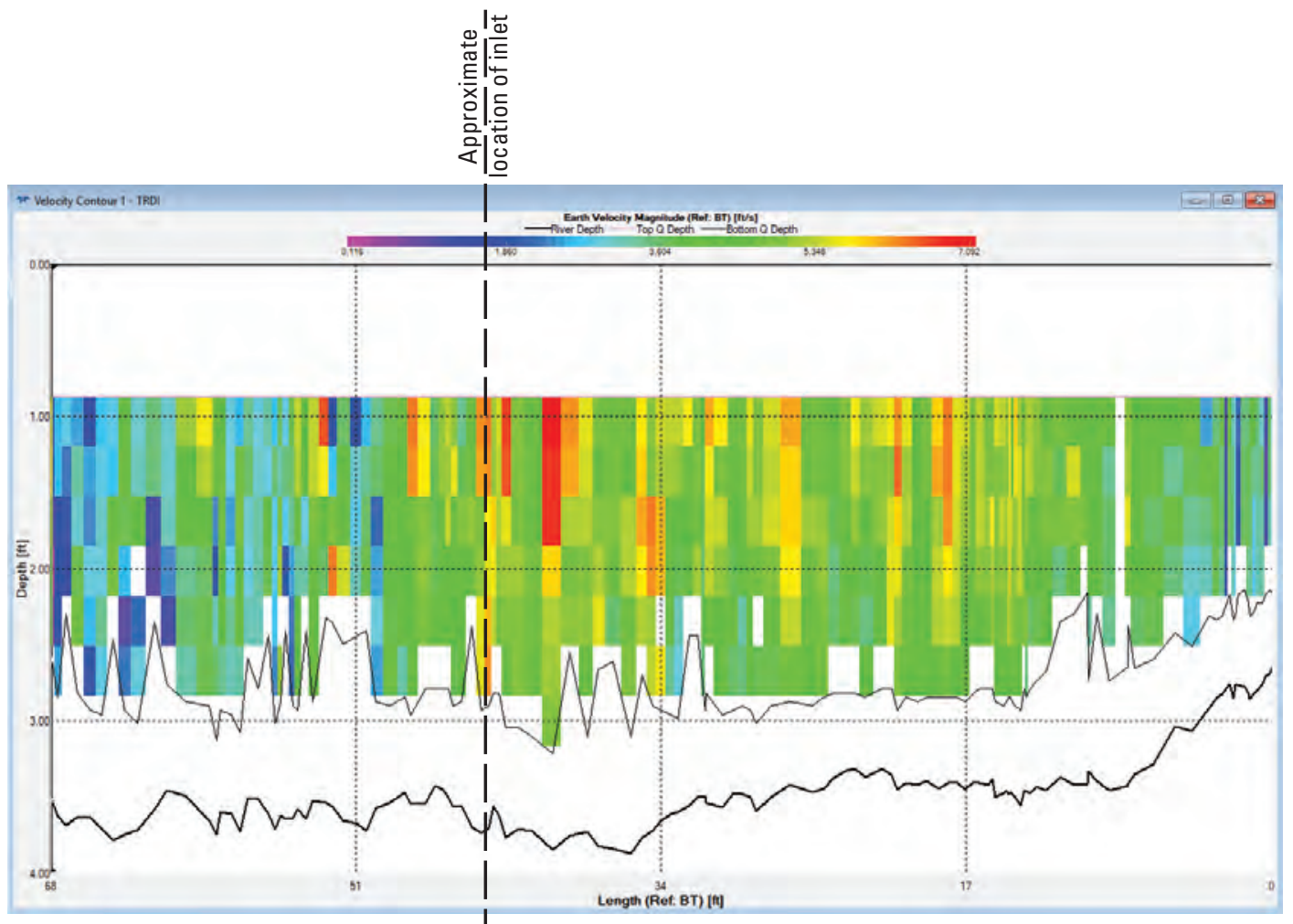

$\boldsymbol{B}$

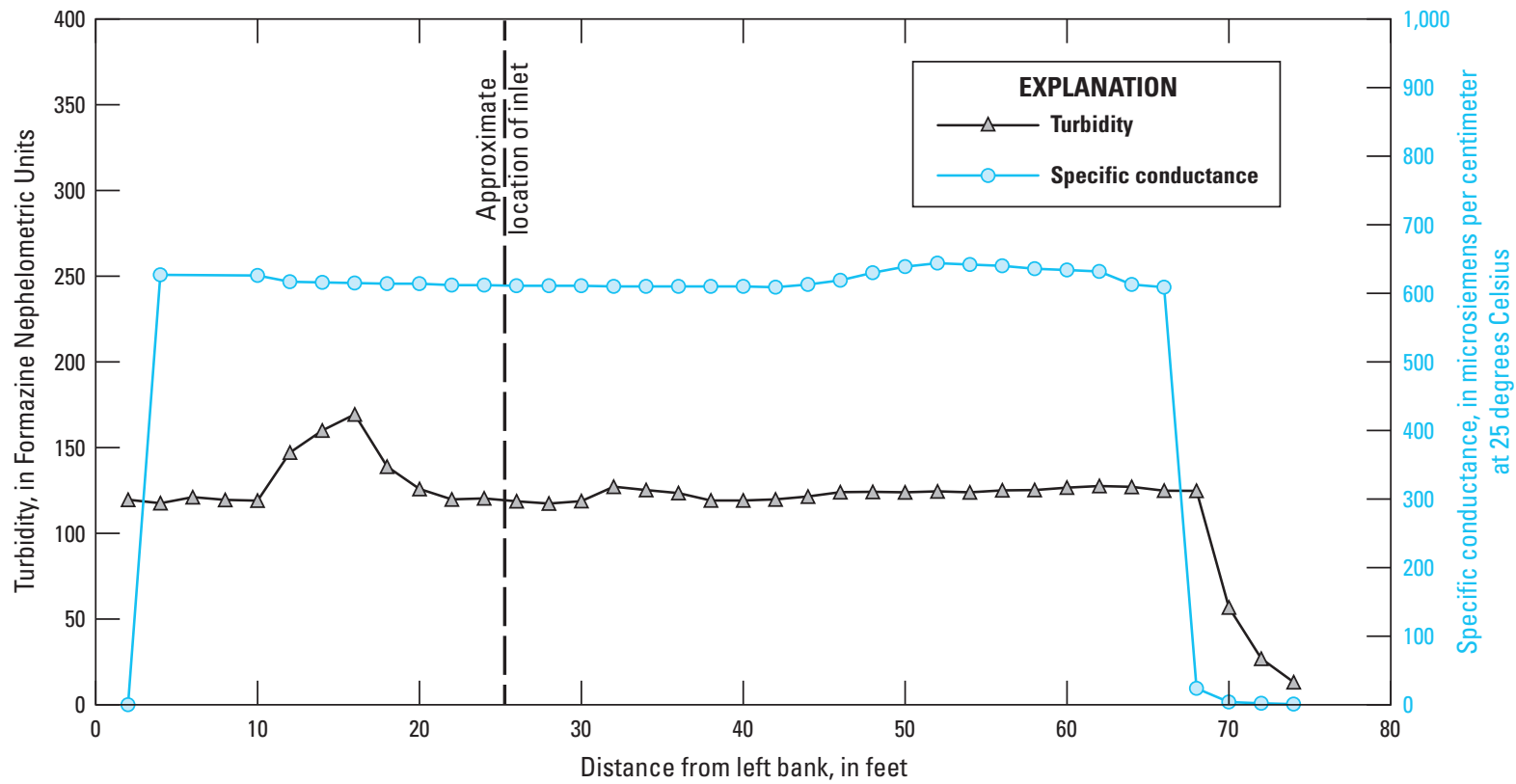

Figure 6. A, Screen capture showing cross-sectional water velocity, and $B$, turbidity and specific conductance measured March 30, 2017, at Beaverdam Creek. 
discharge (high-flow turbidity) were calculated. These substituted turbidity values are listed in table 18. Missing turbidity values were substituted with either the low- or high-flow turbidity replacement values based on the measured discharge at the time. Low and high replacement values for a stream are similar over the years of this study.

3. Missing turbidity values for gaps longer than 3 days were not estimated; these gaps were not included in the calculation of sediment loads. LBDC ultimately contained two long periods of missing turbidity data, the first from January 31 through March 2, 2017 (729 hours), and the second from April 13 through April 24 (265 hours). These data gaps, for the most part, overlap gaps in discharge data.

After the missing turbidity measurements were assigned estimated values, the turbidity record accounted for more than 98 percent of the possible measurements for the year for all streams except LBDC, where only 88 percent of the possible measurements were available. The yearly estimated sediment and sediment-bound loadings discussed farther on in this report are constrained by the coverage of the available continuous turbidity and discharge record.

Linear regression relations were then developed between measured turbidity and SSC. As discussed by Miller and others (2007, 2013), Rasmussen and others (2011), and Helsel and Hirsch (2002), water-quality parameters such as turbidity and SSC can be related to each other through SLR or MLR analysis. "Model datasets" were developed by combining historical measurements of turbidity, SSC, and discharge for 2013-16 available in the USGS NWIS database (U.S. Geological Survey, 2019) with the new measurements made during the present study. The model dataset is included in appendix 3 and is publicly available in ScienceBase as a USGS data release (Wilson, 2019).

From NWIS records beginning in January 2013, the number of SSC data available were 191 for NEB, 144 for Watts Branch, and 156 for Hickey Run. In total, 171 SSC values (beginning in October 2003) were available from NWIS for NWB, and 87 values were available for LBDC (collected in this study beginning in December 2016) for use in the linear regression analysis. These datasets used in the modeling (app. 3, table 3.1; Wilson, 2019) are summarized in table 19.

The continuous turbidity records were found to be "noisy" and contain many short-lived spikes. High variability in turbidity data relates to the rapid changes in discharge and the high measurement rate used (as frequently as 2-minute intervals), and was expected in these small urban streams. To reduce the effect of the noise, the turbidity measurements were averaged over 10- or 12-minute intervals depending on initial measurement rate. The averaged values were then assigned to the reported collection time for each SSC data point in the model dataset. Discharge in Watts Branch, Hickey Run, and, to some degree, LBDC also varied widely, so the instantaneous Q measurements in these streams were also averaged (10- or 12- minute intervals) for use in the regression models. Averaging helps smooth the fluctuations in Q and SSC that occur in these smaller streams and helps compensate for any uncertainty in the reported sample-collection time. Comparing the raw data with the averaged values reveals that, for the most part, the averaged and instantaneous values are nearly identical, especially for NEB and NWB, where stream discharge and turbidity vary more slowly than in the smaller tributaries. Little difference was observed when load calculations were made by using raw or averaged data.

The averaged values of turbidity and Q were then log transformed (base 10) before calculating the best-fit, leastsquares regression models. As discussed by Helsel and Hirsch (2002), log transformation of natural data is needed because measurements of turbidity and SSC in natural environments typically follow a nonnormal distribution. Log transformation of the data also improves the linearity of the relation between measured variables and allows the use of parametric statistical evaluations. However, values predicted in log space require a correction when transformed back to "real" concentrations. The transformed "model datasets" (app. 3, table 3.1; Wilson, 2019) were used to calculate SLR and MLR equations by using Microsoft Office Excel 2010.

Table 19. Summary of stream data used for regression analysis of Anacostia River tributaries.

[ft, feet; $\mathrm{ft}^{3} / \mathrm{s}$, cubic feet per second; FNU, Formazin Nephelometric Units; mg/L, milligrams per liter]

\begin{tabular}{lcccccc}
\hline \multicolumn{1}{c}{ Tributary } & $\begin{array}{c}\text { Range of dates } \\
\text { having data } \\
\text { available }\end{array}$ & $\begin{array}{c}\text { Number of suspended- } \\
\text { sediment concentration } \\
\text { values available }\end{array}$ & $\begin{array}{c}\text { Range in } \\
\text { gage height } \\
\text { (ft) }\end{array}$ & $\begin{array}{c}\text { Range in } \\
\text { discharge } \\
\text { (ft } \mathbf{3} / \mathbf{s})\end{array}$ & $\begin{array}{c}\text { Range in } \\
\text { turbidity } \\
\text { (FNU) }\end{array}$ & $\begin{array}{c}\text { Range of suspended- } \\
\text { sediment concentration } \\
\text { (mg/L) }\end{array}$ \\
\hline Northeast Branch & $2013-2017$ & 191 & $0.62-8.93$ & $1.24-6,900$ & $0.3-1,130$ \\
Northwest Branch & $2003-2017$ & 171 & $1.09-8.23$ & $5.49-9,290$ & $0.60-785$ & $1-2,110$ \\
Beaverdam Creek & $2016-2017$ & 82 & $0.07-6.77$ & $3.23-1,310$ & $2.6-1,070$ & $2-1,700$ \\
Watts Branch & $2013-2017$ & 130 & $4.07-7.79$ & $0.41-888$ & $0.53-700$ & $1-1,560$ \\
Hickey Run & $2013-2017$ & 155 & $1.63-7.69$ & $0.13-893$ & $1.8-430$ & $2-1,270$ \\
\hline
\end{tabular}


The MLR models were calculated as

$$
\begin{aligned}
\log _{10}(S S C)= & \left(\log _{10}(\text { Turbidity }) \times a\right) \\
& +\left(\log _{10}(\mathrm{Q}) \times b\right)-\text { intercept }
\end{aligned}
$$

where

$$
a, b \quad=\quad \text { slope of best-fit line. }
$$

Bias is introduced when log-transformed data are returned to normal arithmetic space. Several procedures are available to correct for this transformation bias. In this study, the retransformed values were corrected by using the Duan bias correction factor (BCF), a value that essentially uses the average difference between the measured and the predicted values in the model dataset used to generate the regression equation (Cohn and others, 1989; Helsel and Hirsch, 2002). $B C F$ is calculated as

$$
B C F=\frac{\sum_{i}^{n} 10^{e i}}{n}
$$

where

$$
\begin{aligned}
e i \quad & \text { difference between the measured and } \\
& \text { predicted SSC in the model dataset, in } \log _{10} \\
n \quad & \text { number of observations. }
\end{aligned}
$$

Suspended-sediment concentrations predicted from the log-transformed turbidity are multiplied by the BCF to obtain the arithmetic value of SSC (Rasmussen and others, 2011). Several metrics can be used to indicate the goodness of the model fit; these metrics include the variance explained by the model ( $\mathrm{r}$ ), the adjusted coefficient of determination $\left(\mathrm{R}^{2}\right)$, the t-statistic for the slope, and the root mean squared error (RMSE). The RMSE provides the model standard percent error (MSPE) in normal arithmetic space. For RMSE expressed in $\log _{10}$ units, the MSPE interval is defined as

$$
\begin{aligned}
\text { Upper MSPE } & =\left(10^{\text {RMSE }}-1\right) \times 100 \\
\text { Lower MSPE } & =\left(1-10^{-\mathrm{RMSE}}\right) \times 100
\end{aligned}
$$

The MSPE provides a normalized measure of the uncertainty in a predicted SSC value resulting from the uncertainty in the model fit. A small difference between the upper and lower MSPE values indicates a model having low uncertainty associated with predicted values. The best-fit regression models for each tributary are listed in table 20 .

The adjusted $\mathrm{R}^{2}$ values and low standardized errors (table 20) indicate the MLR using turbidity and Q adequately explains the variance in the model dataset, and that these models provide statistically significant predicted SSC. The large t-ratios and small p-statistics for each model support that the slopes are significant and at any reasonable probability differ from 0 . In all cases, excellent linear relations are indicated. The Durban-Watson values indicate a positive correlation between turbidity and discharge, which is confirmed visually in plots of these parameters. As expected, turbidity is high when discharge is high. The $\mathrm{t}$ - and $\mathrm{p}$-statistics calculated for the various intercepts indicate that for the LBDC and Hickey Run models, the intercepts may not be significantly different from 0 . The meaning of nonzero intercepts in regression modeling of turbidity is unclear; however, regression lines between turbidity and SSC typically do not pass through the origin because turbidity can be affected by water color and the presence of certain dissolved compounds and oils (Sutherland and others, 2000; Anderson, 2005; Rasmussen and others, 2011). Plots of the regression residuals, defined as the difference between measured and predicted SSC values, were found to follow a normal distribution in all streams, and there is no evidence of heteroscedasticity (nonconstant variance) in the data. The log-transformed models using both variables (and Q), therefore, are good predictors of SSC.

Residuals provide a measure of the accuracy of the model through the RMSE and corresponding MSPE. The best-fit regression lines have standard errors of 14 to 27 percent. Plots of the log-transformed measured and predicted SSC (fig. 7) are used to visually assess the accuracy of the models. Overall, the plots show a good correspondence between the predicted and measured SSC; however, no systematic variation from the logarithmic trend is observed, especially at higher SSC. Considerable scatter is shown in the data from NWB when SSC is $<10 \mathrm{mg} / \mathrm{L}$. Low SSC values correspond to base flow, so inaccuracies in predicted SSC during low flow have little effect on total sediment loads. The plot for Hickey Run (fig. 7E) indicates scatter in data at higher SSCs, corresponding to higher values of turbidity and discharge. The scatter in the Hickey Run data appears greater than that in the data from the other tributaries. This scatter may be related to the presence of the sediment trap located just upstream from the sampling station. Maintenance of this trap has been reported to be inconsistent over the years; it is possible that the trap affects the relation between SSC and discharge. Oil films commonly observed in Hickey Run during fieldwork also may affect the relation between turbidity and SSC in this stream.

SLR and MLR equations are valid only over the range of turbidity and discharge represented in the model dataset. The data used to develop the model overlap with the measured turbidity and discharge measured in the streams during 2017 (table 21). The model dataset covers a wide range in turbidity $(<1-1,130 \mathrm{FNU})$ and peak discharge (from $893 \mathrm{ft}^{3} / \mathrm{s}$ in Hickey Run to $6,900 \mathrm{ft} / 3 / \mathrm{s}$ in NEB). Discharge in LBDC exceeded the maximum Q in the model dataset for only 60 minutes, and in Hickey Run for only 46 minutes. The sensors used in this study can measure turbidity accurately up to 1,700 FNU (Anderson, 2005). The model dataset includes SSCs measured over almost the entire range of continuous turbidity recorded at each station. During 2017, the cumulative times that turbidity exceeded that in the model were $0 \mathrm{~min}$ utes for NEB, 30 minutes for NWB, 80 minutes for LBDC, 278 minutes for Watts Branch, and 0 minutes for Hickey Run. With few exceptions (which generally occurred in 2014), the equations developed from the model dataset cover the $\mathrm{Q}$ and turbidity values measured in these tributaries. 
Table 20. Regression models for predicting suspended-sediment concentrations in the Anacostia River tributaries.

[SSC, suspended-sediment concentration, in milligrams per liter; FNU, Formazin Nephelometric Units; adjusted R², adjusted coefficient of determination; SE, standard error of the regression; BCF, bias correction factor, MSPE, model standard percent error, in percent; Q, discharge; <, less than; na, not applicable; NEB, Northeast Branch; NWB, Northwest Branch; LBDC, Beaverdam Creek; WB, Watts Branch; HR, Hickey Run]

\begin{tabular}{|c|c|c|c|c|c|c|c|c|c|c|c|}
\hline Watershed & $\begin{array}{l}\text { Number of } \\
\text { observations }\end{array}$ & Equation ${ }^{1}$ & $\begin{array}{l}\text { Adjusted } \\
\qquad \mathbf{R}^{2}\end{array}$ & $\begin{array}{l}\text { t-stat slope } \\
\text { for turbidity }\end{array}$ & $\begin{array}{l}\text { P-slope } \\
\text { for turbidty }\end{array}$ & $\begin{array}{l}\text { t-stat slope } \\
\quad \text { for } 0\end{array}$ & $\begin{array}{l}\text { P-slope } \\
\text { for } 0\end{array}$ & SE & $\mathrm{BCF}$ & $\begin{array}{c}\text { Lower } \\
\text { MSPE } \\
\text { (percent) }\end{array}$ & $\begin{array}{c}\text { Upper } \\
\text { MSPE } \\
\text { (percent) }\end{array}$ \\
\hline \multicolumn{12}{|c|}{ Multiple linear regression } \\
\hline NEB & 177 & $\begin{aligned} \log _{10}(\text { SSC }) & =\log _{10}(\text { turbidity }) \times 0.96207+ \\
\log _{10}(\mathrm{Q}) & \times 0.17102-0.2531\end{aligned}$ & 0.93 & 25.4 & $<0.001$ & 3.17 & 0.002 & 0.202 & 1.04 & 37.0 & 58.8 \\
\hline NWB & 171 & $\begin{aligned} \log _{10}(\mathrm{SSC}) & =\log _{10}(\text { turbidity }) \times 0.8430+ \\
\log _{10}(\mathrm{Q}) & \times 0.2553-0.09265\end{aligned}$ & 0.95 & 22.9 & $<0.001$ & 6.71 & $<0.001$ & 0.192 & 1.10 & 35.5 & 55.6 \\
\hline LBDC & 87 & $\begin{aligned} \log _{10}(\mathrm{SSC}) & =\log _{10}(\text { turbidity }) \times 0.764326+ \\
\log _{10}(\mathrm{Q}) & \times 0.355299-0.18901\end{aligned}$ & 0.94 & 12.0 & $<0.001$ & 6.13 & $<0.001$ & 0.144 & 1.05 & 27.9 & 38.7 \\
\hline WB & 144 & $\begin{aligned} \log _{10}(\mathrm{SSC}) & =\log _{10}(\text { turbidity }) \times 0.69084+ \\
\log _{10}(\mathrm{Q}) & \times 0.43444+0.06402\end{aligned}$ & 0.89 & 7.07 & $<0.001$ & 5.72 & $<0.001$ & 0.262 & 1.20 & 44.6 & 85.7 \\
\hline HR & 155 & $\begin{aligned} \log _{10}(\mathrm{SSC}) & =\log _{10}(\text { turbidity }) \times 0.64896+ \\
\log _{10}(\mathrm{Q}) & \times 0.264817+0.4457\end{aligned}$ & 0.82 & 12.2 & $<0.001$ & 7.86 & $<0.001$ & 0.271 & 1.21 & 46.4 & 85.7 \\
\hline \multicolumn{12}{|c|}{ Simple linear regression } \\
\hline NEB & 176 & $\log _{10}(\mathrm{SSC})=\log _{10}($ turbidity $) \times 1.1345-0.1576$ & 0.92 & 45.3 & $<0.001$ & na & na & 0.207 & 0.74 & 37.8 & 60.8 \\
\hline NWB & 171 & $\log _{10}(\mathrm{SSC})=\log _{10}($ turbidity $) \times 1.0565+0.13911$ & 0.94 & 50.7 & $<0.001$ & na & na & 0.215 & 1.10 & 38.9 & 63.7 \\
\hline LBDC & 82 & $\log _{10}(\mathrm{SSC})=\log _{10}($ turbidity $) \times 1.0672-0.0545$ & 0.91 & 28.2 & $<0.001$ & na & na & 0.185 & 1.12 & 31.4 & 45.7 \\
\hline WB & 130 & $\log _{10}(\mathrm{SSC})=\log _{10}($ turbidity $) \times 1.205-0.3195$ & 0.85 & 28.2 & $<0.001$ & na & na & 0.293 & 1.28 & 48.8 & 95.9 \\
\hline HR & 155 & $\log _{10}(\mathrm{SSC})=\log _{10}($ turbidity $) \times 0.9962+0.1404$ & 0.75 & 21.4 & $<0.001$ & na & na & 0.321 & 1.43 & 52.0 & 108 \\
\hline
\end{tabular}

'In regression equations, discharge is in cubic feet per second and turbidity is in FNU. 

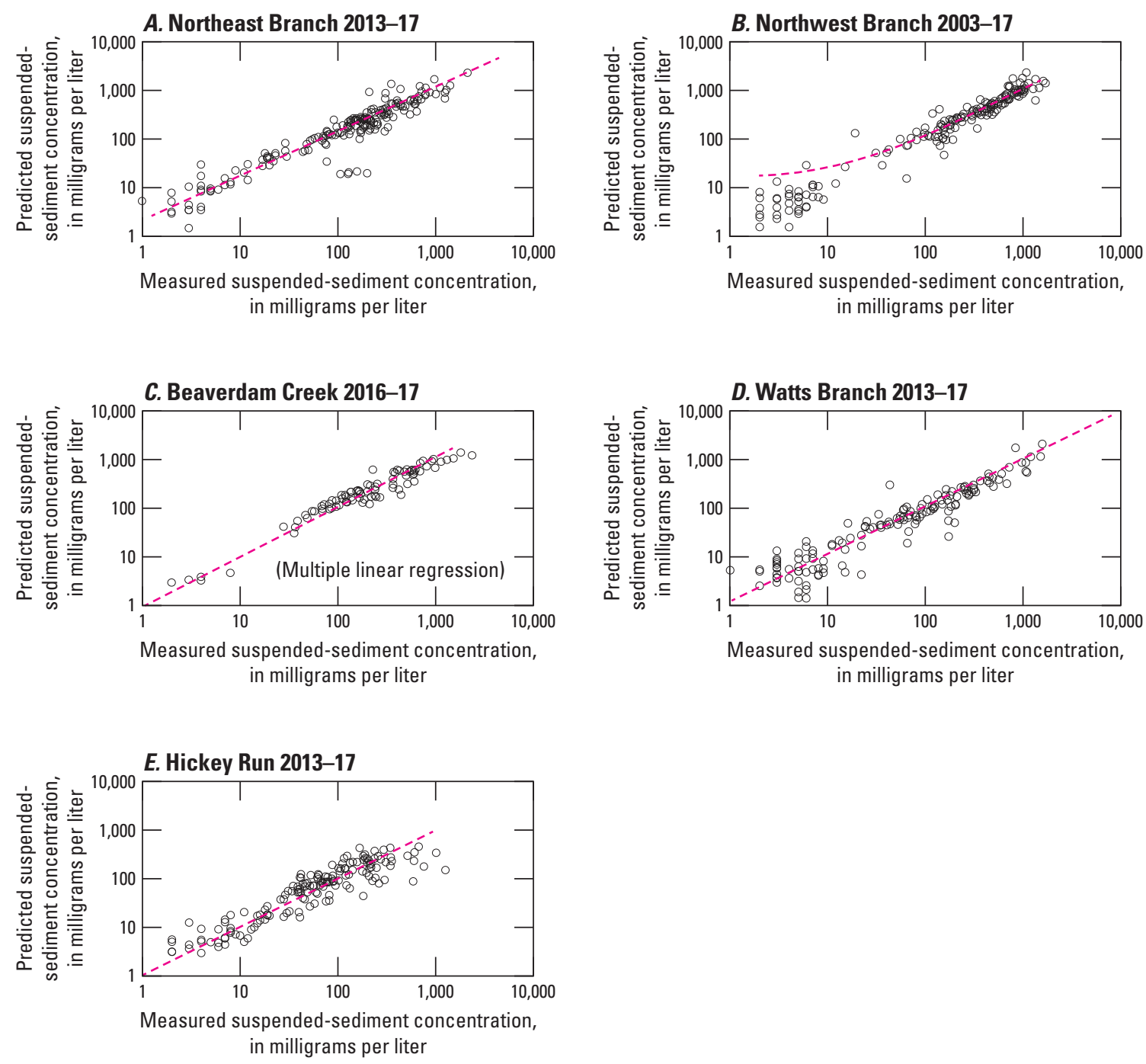

Figure 7. Relation between the measured suspended-sediment concentrations in the model dataset and predicted concentrations in $A$, Northeast Branch, $B$, Northwest Branch, $C$, Beaverdam Creek, $D$, Watts Branch, and $E$, Hickey Run. 
Table 21. Summary of yearly discharge and turbidity in the continuous records for Anacostia River tributaries compared with model dataset.

[ $\mathrm{ft}^{3} / \mathrm{s}$, cubic feet per second; FNU, Formazin Nephelometric Units; min, minutes; --, not applicable]

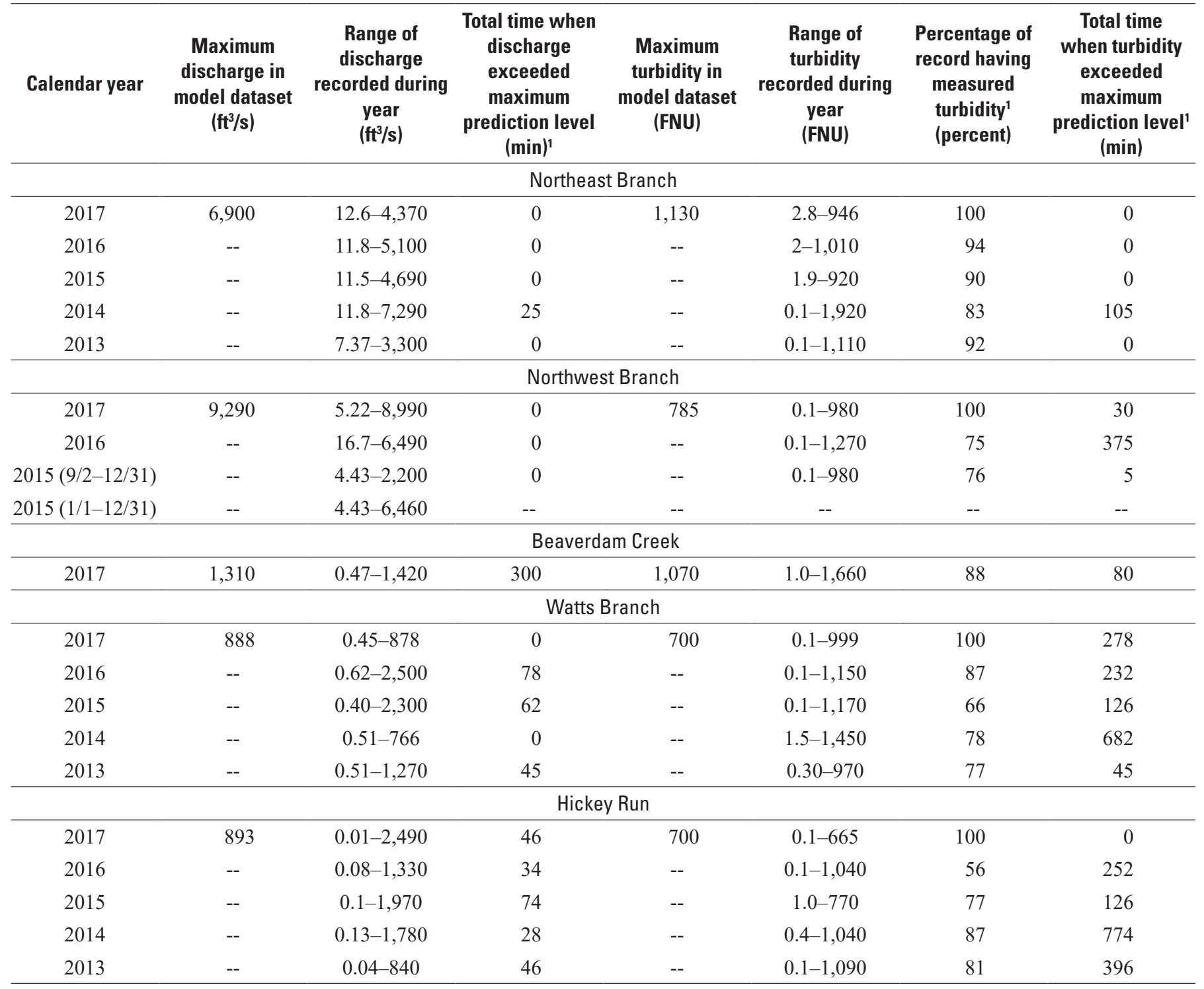

\footnotetext{
${ }^{1}$ The total time of discharge and turbidity in record is determined by using the dataset having all missing values replaced by estimated values.
} 
As an example of the agreement between measured and predicted SSC, plots of SSC (measured and predicted from turbidity) and Q during the storm sampled on March 30, 2017, were prepared (fig. 8). Although only a few SSCs were obtained during each storm, the measured and predicted concentrations generally agree well. The model for NEB appears to underpredict the peak SSC, possibly because the peak discharge recorded during this storm was low.

A high degree of confidence can be placed in the predicted SSCs and, therefore, the calculated sediment loads, considering the (1) high rate at which turbidity and discharge were measured, (2) small percentage of time that turbidity and discharge exceeded the range in the model dataset, (3) small cumulative time that required using estimated values of turbidity or discharge or during which values were missing completely, and (4) high degree of fit of the regression equations.

By using the continuous discharge and turbidity and the MLR equations described above (table 20), SSCs and sediment loadings in the gaged tributaries were calculated for each time step. The sediment loadings for Watts Branch were used to estimate loadings in the ungaged tributaries on the basis of relative basin area. These sediment loadings were then multiplied by the averaged chemical concentration to provide chemical loadings in each tributary. The two sections that follow describe the results of these calculations.

\section{Sediment Loads in Gaged Tributaries}

The MLR equations (table 20) were used to predict SSCs for each measurement of turbidity and discharge in the streams. These instantaneous loads were summed to obtain total loads for 2013 through 2017. Loadings for NEB could only be computed back to 2015; those for LBDC could only be computed for 2017. Yearly loadings are shown in table 22, and summaries of loadings for the individual storm events are listed in appendix 1 (table 1.1). All of these sediment loadings were calculated using data available in NWIS.

The total sediment loading for $2017,3.10 \times 10^{7} \mathrm{~kg} / \mathrm{yr}$, was distributed among the streams as follows: 33 percent $\left(1.02 \times 10^{7} \mathrm{~kg} / \mathrm{yr}\right)$ from NEB, 50 percent $\left(1.55 \times 10^{7} \mathrm{~kg} / \mathrm{yr}\right)$ from NWB, 14 percent $\left(4.45 \times 10^{6} \mathrm{~kg} / \mathrm{yr}\right)$ from LBDC, 2 percent $\left(5.62 \times 10^{5} \mathrm{~kg} / \mathrm{yr}\right)$ from Watts Branch, and 1 percent $\left(2.82 \times 10^{5} \mathrm{~kg} / \mathrm{yr}\right)$ from Hickey Run. The yearly loadings represent daily loadings of 85,000 kilograms per day $(\mathrm{kg} / \mathrm{d})$, with $27,900 \mathrm{~kg} / \mathrm{d}$ from NEB, $42,500 \mathrm{~kg} / \mathrm{d}$ from NWB, $12,200 \mathrm{~kg} / \mathrm{d}$ from LBDC, $1,650 \mathrm{~kg} / \mathrm{d}$ from Watts Branch, and $773 \mathrm{~kg} / \mathrm{d}$ from Hickey Run.

Yearly sediment loadings vary in response to yearly precipitation, although the relation is not always straightforward. Loads in NEB, for example, ranged from a minimum of $1.00 \times 10^{7} \mathrm{~kg}$ in 2013 , when total precipitation was $34.7 \mathrm{in}$, to a maximum of $3.63 \times 10^{7} \mathrm{~kg}$ in 2014 , when 42.9 in. of precipitation fell-a range of $2.63 \times 10^{7}$ $\mathrm{kg}$. In Watts Branch, a much smaller basin, loads ranged from a minimum of $5.36 \times 10^{5} \mathrm{~kg}$ in 2013 to a maximum of $1.35 \times 10^{6} \mathrm{~kg}$ in 2016 - a range of $8.14 \times 10^{5} \mathrm{~kg} / \mathrm{yr}$.

Sediment yields varied slightly among the basins. During 2017, sediment yields were $1.40 \times 10^{5}$ kilograms per year per square mile $\left(\mathrm{kg} / \mathrm{yr} / \mathrm{mi}^{2}\right)$ for NEB, $3.13 \times 10^{5} \mathrm{~kg} / \mathrm{yr} / \mathrm{mi}^{2}$ for NWB, $3.01 \times 10^{5} \mathrm{~kg} / \mathrm{yr} / \mathrm{mi}^{2}$ for LBDC, $1.67 \times 10^{5} \mathrm{~kg} / \mathrm{yr} / \mathrm{mi}^{2}$ for Watts Branch, and $2.79 \times 10^{5} \mathrm{~kg} / \mathrm{yr} / \mathrm{mi}^{2}$ for Hickey Run. The yields in NWB, LBDC, and Hickey Run are similar, which was unexpected because of the differences in the physical characteristics of the basins. Hickey Run flows underground for most of its length, and likely gains much of its sediment as street grit in runoff; the basins of LBDC and NWB differ greatly in area (14.9 and $49.4 \mathrm{mi}^{2}$, respectively).

The uncertainty in SSC introduced by the use of regression analysis to compute sediment concentrations is propagated to the loadings. The MSPEs listed in table 20 provide one measure of the uncertainty in predicted SSC. For example, the MSPE for NEB ranges from 37.0 to 58.8 percent, so a predicted concentration of $100 \mathrm{mg} / \mathrm{L}$ for SSC may fall anywhere between 63 and $159 \mathrm{mg} / \mathrm{L}$. Statistical measures such as the 95-percent confidence interval were calculated and show a much larger confidence range around predicted SSC than that indicated by the MSPE. The potential sources of uncertainty in the sediment and chemical loadings in this report include uncertainty in dry weight of sediment submitted to the analytical laboratory, uncertainty in SSCs in the streamwater during low-flow sampling, the use of a point measurement of turbidity to represent the entire cross section of the stream, the predictions made by linear-regression models to estimate SSC, and uncertainty in estimated discharge (uncertainty in the relation between stage and discharge or stage and velocity). The effects of uncertainty in moisture content of sediment were discussed previously.

\section{Sediment Loads in Ungaged Tributaries}

Sediment loads in the ungaged tributaries were determined from those in Watts Branch, adjusted by using the basin area normalized to the area of the Watts Branch Basin (table 23). Loads from these small tributaries in 2017 totaled $3.5 \times 10^{5} \mathrm{~kg} / \mathrm{yr}$, slightly greater than the sediment loading from Watts Branch. The largest contributions were from Nash Run (34 percent) and Fort DuPont Creek (32 percent), reflecting the similarity in basin areas. Nash Run has the largest basin area of these tributaries, and Fort DuPont Creek has the steepest gradient ( $240 \mathrm{ft} / 1.9 \mathrm{mi}$, or 0.024). Because considerable erosion is evident in the Fort DuPont Creek channel north of Minnesota Avenue, the estimated load presented here may underestimate the actual load.

\section{Concentrations of Sediment-Bound Contaminants}

This section discusses the chemical data and the calculation of the COC loadings. The representativeness of the storm 


\section{A. Northeast Branch}
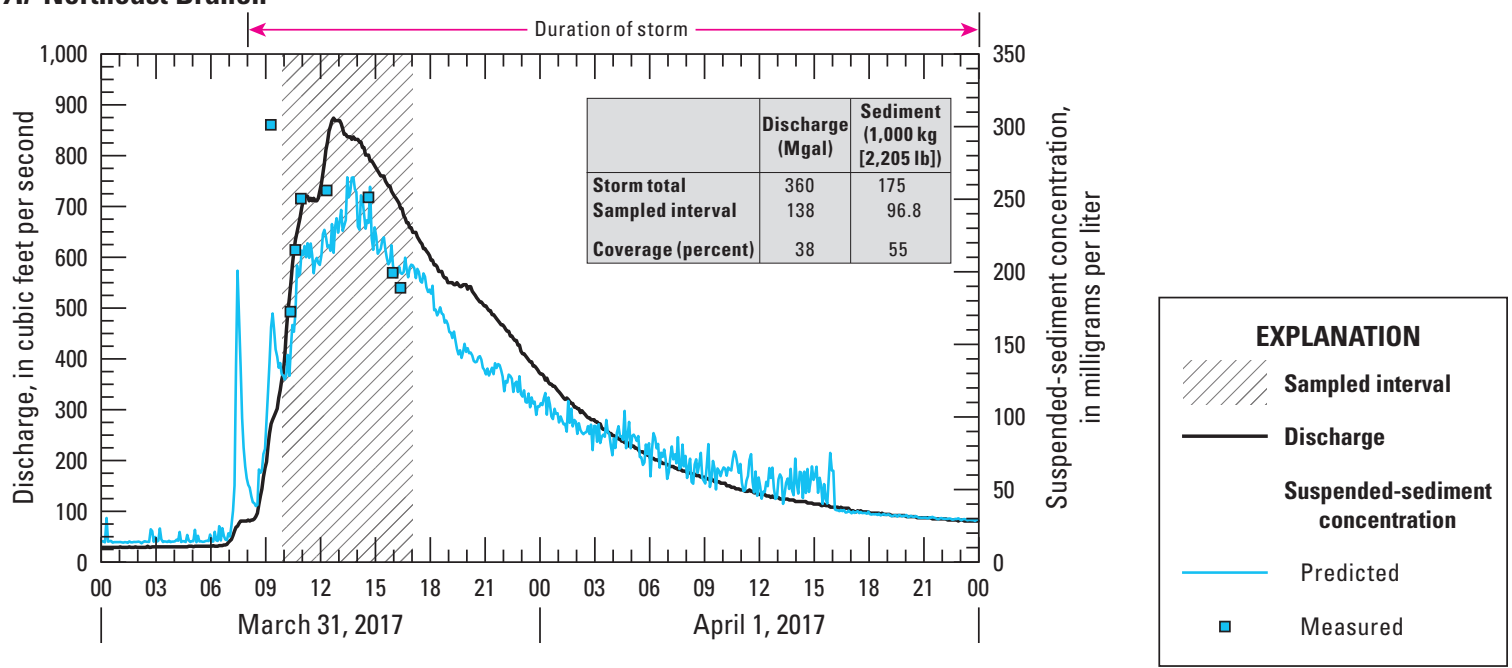

\section{B. Northwest Branch}

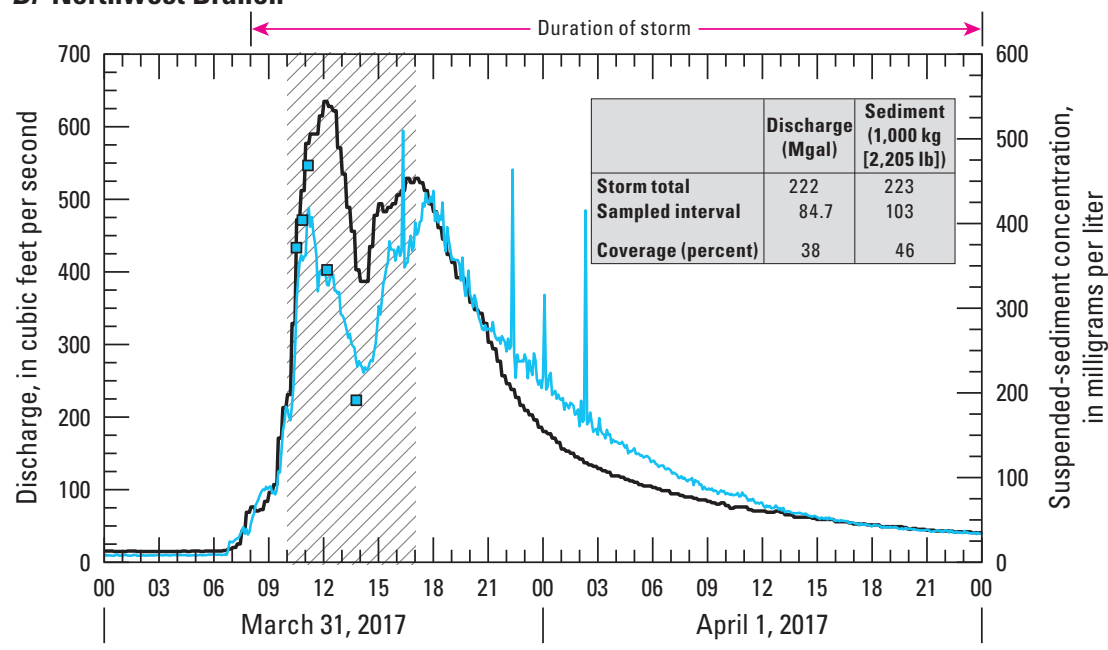

\section{Beaverdam Creek}

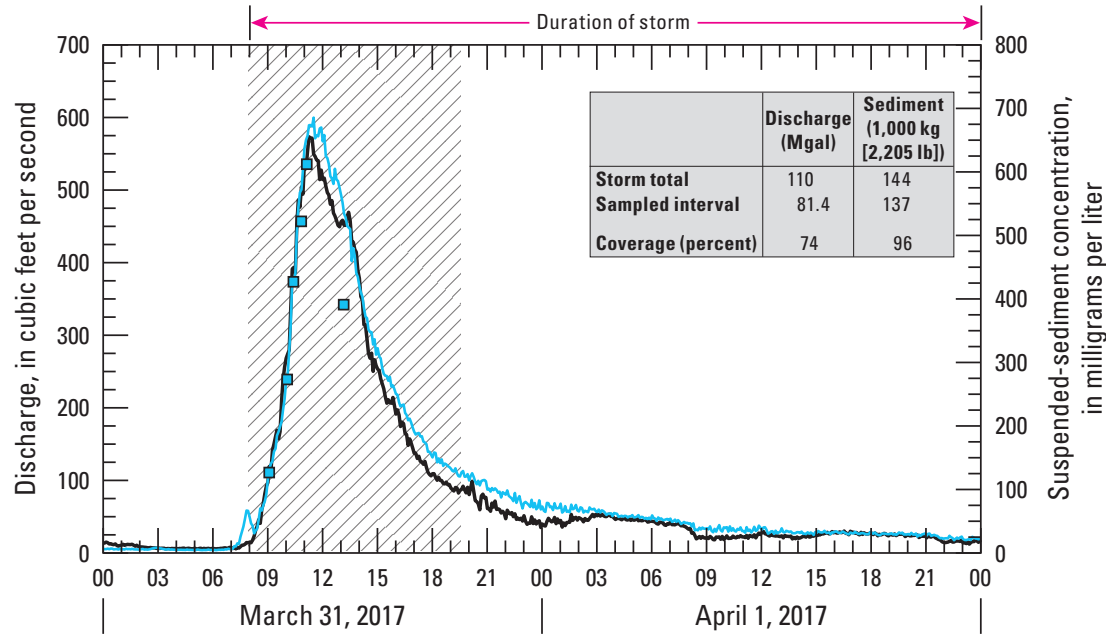

Figure 8. Discharge and measured and predicted suspended-sediment concentrations in $A$, Northeast Branch, $B$, Northwest Branch, $C$, Beaverdam Creek, D, Watts Branch, and $E$, Hickey Run for the storm beginning March 31, 2017. (Mgal, million gallons; $\mathrm{kg}$, kilograms; lbs, pounds) 

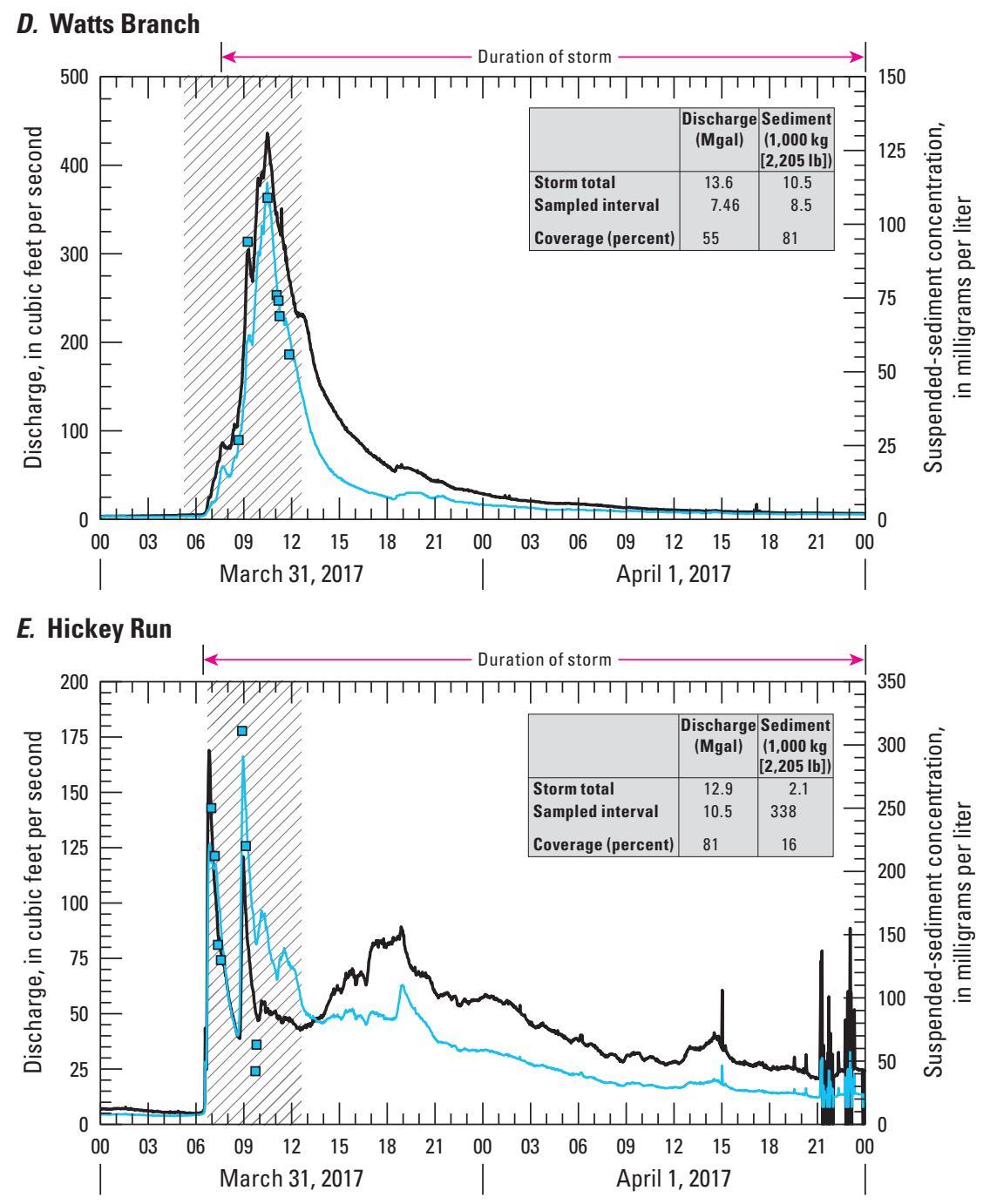

Figure 8. Hydrographs showing discharge and measured and predicted suspended-sediment concentrations in $A$, Northeast Branch, $B$, Northwest Branch, $C$, Beaverdam Creek, $D$, Watts Branch, and $E$, Hickey Run for the storm beginning March 31, 2017. (Mgal, million gallons; kg, kilograms; Ibs, pounds)_Continued 
Table 22. Annual discharge and predicted sediment load for tributaries to the Anacostia River.

$\left[\mathrm{ft}^{3} / \mathrm{s}\right.$, cubic feet per second; FNU, Formazin Nephelometric Units; Mgal, million gallons; L, liters; kg/yr, kilograms per year; $\mathrm{kg} / \mathrm{d}$, kilograms per day; $\mathrm{kg} / \mathrm{yr} / \mathrm{mi}^{2}$, kilograms per year per square mile; --, not applicable]

\begin{tabular}{|c|c|c|c|c|c|c|c|c|}
\hline Calendar year & $\begin{array}{c}\text { Maximum } \\
\text { discharge } \\
\left(\mathrm{ft}^{3} / \mathrm{s}\right)\end{array}$ & $\begin{array}{c}\text { Average } \\
\text { discharge } \\
\left(\mathrm{ft}^{3} / \mathrm{s}\right)\end{array}$ & $\begin{array}{c}\text { Maximum } \\
\text { turbidity } \\
\text { (FNU) }\end{array}$ & $\begin{array}{c}\text { Total } \\
\text { discharge } \\
\text { (Mgal) }\end{array}$ & $\begin{array}{c}\text { Total } \\
\text { discharge } \\
\text { (L) }\end{array}$ & $\begin{array}{c}\text { Sediment } \\
\text { load } \\
(\mathrm{kg} / \mathrm{yr})\end{array}$ & $\begin{array}{c}\text { Sediment } \\
\text { load } \\
(\mathrm{kg} / \mathrm{d})\end{array}$ & $\begin{array}{c}\text { Sediment } \\
\text { yield } \\
\left(\mathrm{kg} / \mathrm{yr} / \mathrm{mi}^{2}\right)\end{array}$ \\
\hline \multicolumn{9}{|c|}{ Northeast Branch } \\
\hline 2017 & 4,370 & 67.4 & 1,150 & 15,800 & $5.97 \times 10^{10}$ & $1.02 \times 10^{7}$ & 27,900 & $1.40 \times 10^{5}$ \\
\hline 2016 & 5,010 & 92.5 & 1,010 & 18,900 & $7.14 \times 10^{10}$ & $1.02 \times 10^{7}$ & 28,000 & $1.35 \times 10^{5}$ \\
\hline 2015 & 4,690 & 103 & 920 & 24,800 & $9.37 \times 10^{10}$ & $1.63 \times 10^{7}$ & 44,600 & $2.16 \times 10^{5}$ \\
\hline 2013 & 3,300 & 72.6 & 1,110 & 17,700 & $7.00 \times 10^{10}$ & $1.00 \times 10^{7}$ & 27,400 & $1.32 \times 10^{5}$ \\
\hline Average & 1,930 & 91.5 & -- & 21,000 & $8.00 \times 10^{10}$ & $1.66 \times 10^{7}$ & 45,500 & $2.20 \times 10^{5}$ \\
\hline \multicolumn{9}{|c|}{ Northwest Branch } \\
\hline 2017 & 8,990 & 47.1 & 985 & 10,900 & $4.15 \times 10^{10}$ & $1.55 \times 10^{7}$ & 42,300 & $3.13 \times 10^{5}$ \\
\hline Average (2016-17) & 7,740 & 51.6 & -- & 10,600 & $4.01 \times 10^{10}$ & $1.05 \times 10^{7}$ & 36,400 & $2.13 \times 10^{5}$ \\
\hline \multicolumn{9}{|c|}{ Beaverdam Creek } \\
\hline 2017 & 1,840 & 19.8 & 1,660 & 4,580 & $1.73 \times 10^{10}$ & $4.45 \times 10^{6}$ & 12,200 & $3.01 \times 10^{5}$ \\
\hline \multicolumn{9}{|c|}{ Watts Branch } \\
\hline 2017 & 878 & 2.98 & 999 & 660 & $2.49 \times 10^{9}$ & $5.62 \times 10^{5}$ & 1,540 & $2.70 \times 10^{5}$ \\
\hline 2016 & 2,500 & 3.72 & 1,150 & 757 & $2.86 \times 10^{9}$ & $1.35 \times 10^{6}$ & 368 & $4.02 \times 10^{5}$ \\
\hline 2015 & 2,300 & 3.91 & 1,170 & 960 & $3.63 \times 10^{9}$ & $1.01 \times 10^{6}$ & 2,780 & $3.01 \times 10^{5}$ \\
\hline 2014 & 766 & 5.70 & 1,450 & 1,530 & $5.79 \times 10^{9}$ & $1.28 \times 10^{6}$ & 3,680 & $3.81 \times 10^{5}$ \\
\hline 2013 & 1,270 & 3.65 & 970 & 1,040 & $3.94 \times 10^{9}$ & $5.36 \times 10^{5}$ & 1,460 & $1.60 \times 10^{5}$ \\
\hline 2013 & 1,840 & 1.40 & 1,090 & 435 & $1.65 \times 10^{9}$ & $2.89 \times 10^{5}$ & 792 & $2.86 \times 10^{5}$ \\
\hline Average & 1,830 & 1.87 & -- & 450 & $1.68 \times 10^{9}$ & $3.55 \times 10^{5}$ & 821 & $3.52 \times 10^{5}$ \\
\hline
\end{tabular}

${ }^{1}$ Turbidity in Northwest Branch was measured beginning on September 2, 2015 (one-third of the year). The data in this row are normalized to 1 year and are thought to underestimate the true value.

${ }^{2}$ The 2015 record was normalized to 365 days. 
Table 23. Estimated sediment loads in ungaged tributaries to the Anacostia River, calendar year 2017.

[mi², square miles; kg, kilograms; kg/d, kilograms per day; --, not applicable]

\begin{tabular}{lcccc}
\hline \multicolumn{1}{c}{ Tributary } & $\begin{array}{c}\text { Basin } \\
\text { area } \\
\left(\mathbf{m i}^{2}\right)\end{array}$ & $\begin{array}{c}\text { Relative } \\
\text { area }^{1}\end{array}$ & $\begin{array}{c}\text { Sediment } \\
\text { load } \\
\mathbf{( k g )}\end{array}$ & $\begin{array}{c}\text { Sediment } \\
\text { load } \\
(\mathbf{k g} / \mathbf{d})\end{array}$ \\
\hline Watts Branch & 3.36 & 1 & $1.7 \times 10^{5}$ & 1,540 \\
Nash Run & 0.71 & 0.21 & $1.2 \times 10^{5}$ & 330 \\
Popes Branch & 0.34 & 0.14 & $6.2 \times 10^{4}$ & 170 \\
Fort DuPont Creek & 0.68 & 0.20 & $1.1 \times 10^{5}$ & 300 \\
Fort Stanton Creek & 0.22 & 0.07 & $5.6 \times 10^{4}$ & 150 \\
Total ungaged tributaries $^{2}$ & 2.09 & -- & $3.5 \times 10^{5}$ & 950 \\
\hline
\end{tabular}

${ }^{1}$ Relative area is the ratio of basin area to area of Watts Branch Basin.

${ }^{2}$ Total not including Watts Branch.

samples is evaluated by using sampling metrics including the coverage of each storm sample, the mass of sediment obtained for analysis, and the cross-channel representativeness of the point samples. The chemistry of PCBs and PAHs is described briefly before the loadings are reported. Pesticides and metals are considered separately from PCBs and PAHs.

\section{Representativeness of Samples}

The third dataset required to calculate loadings is representative concentrations of the COCs. Unlike turbidity and discharge, which were measured continuously, trace organic chemicals were represented by only a few samples. The sampling scheme was designed to produce, to the extent possible, representative chemistries in the suspended sediment throughout each storm event and season. Three characteristics of the sampling that describe the representativeness of the samples include (1) the seasonal distribution of the samples collected, (2) the percentage of each storm hydrograph represented by the collected aliquots and the mass of sediment obtained for analysis, and (3) the location in the stream where point samples were collected.

With one exception (Hickey Run, sampled in July), sampling was conducted during spring, fall, and winter when algae populations in the streams were lowest. During summer, algae were abundant in the larger streams. Because the small, ungaged tributaries were quickly flushed or were dry during the summer months, algae were not a factor and sampling could be conducted.

Sampling seasonally helps capture the variability in sediment chemistry that may result from various environmental factors. These factors include, among others, the length of time without precipitation preceding a storm (fewer storms in the fall allow fine-grained material to build up in the streams and oils and greases to accumulate on road surfaces); temperature (higher temperatures enhance volatilization of organic chemicals from water and sediment); road salting in winter (resulting in increased ionic strength of surface runoff); and periods of increased input of organic matter, such as leaves and other plant debris.

Coverage of samples over the storm hydrograph was then considered. Upon completion of each sampling event, the discharge and turbidity records and sampling times were plotted to evaluate the LV composites. Indicating the times when discrete suspended sediment and POC samples were collected on the turbidity record aided in selecting samples for analysis, thereby ensuring a full range of turbidity, SSC, and Q values for the regression analysis described earlier. The total discharge and total sediment mass transported during each storm were calculated, and the subsequent coverage represented by the sampling was calculated. Metrics including total volume, duration, and sediment load for each storm are listed in appendix 1 (table 1.3).

An example of these calculations is shown in figure 8, which shows hydrographs for the gaged tributaries for the storm beginning March 31, 2017. Discharge, predicted SSC, and the sampled interval are shown for the tributaries. At $\mathrm{NEB}$, the total discharge and sediment load during the storm were calculated to be $360.6 \mathrm{Mgal}$ and $1.84 \times 10^{5} \mathrm{~kg}$, respectively. In this case, the sampling accounted for 42 percent of the discharge, whereas sediment was collected from 57 percent of the total sediment mass ultimately transported. In LBDC, the storm produced $135,000 \mathrm{~kg}$ of sediment and $82 \mathrm{Mgal}$ of water; sampling covered more than 98 percent of the discharge and the sediment load. Turbidity in the stream returned to prestorm levels on April 5, well after the return of the tidal cycling (not shown). Between the termination of sampling and the return of turbidity to prestorm levels, an additional $6,500 \mathrm{~kg}$ of sediment was transported, a negligible percentage of the total sediment moved during the storm.

Storm sampling in this study covered a range from 10 percent (May 24, 2017, in NEB) to 98 percent (May 5, 2017, in NWB) of the sediment mass transported in each storm. The low coverage in the NEB sample from March was the result of a power failure that stopped the discrete sampler but not the LV composite sampler. If this sample is not considered, sample coverage averaged substantially more than 50 percent for sediment, with higher coverages generally found in the smaller tributaries. On the basis of these metrics, the LV composite samples are considered to adequately represent the sediment in the various streams during the storms.

The total mass of sediment collected for analysis is a second metric describing the representativeness of the samples. The mass needed to be sufficient so the lowest possible method detection levels (MDLs) could be obtained; low MDLs reduce the number of nondetects and allow comparisons to be made among the samples and tributaries. A target mass of $1 \mathrm{~g}$ of sediment per sample was used in this study; actual masses obtained are listed in table 6. Generally, from 20 to $60 \mathrm{~g}$ of sediment was obtained during storm sampling, whereas from 1.5 to $5 \mathrm{~g}$ of sediment was obtained during 
low-flow sampling. The maximum mass of sediment collected under low-flow conditions, an estimated $12 \mathrm{~g}$, was collected from NEB on November 30, 2017, by streamside filtration.

Another factor affecting the representativeness of a storm sample is the location in the cross section of the stream where the aliquots were obtained, and how well this point represents the cross-channel variability in sediment distribution and water velocity (Wagner and others, 2006). As described previously, the locations chosen for the inlet nozzle for the autosamplers (and turbidity sensors) were considered to produce samples representative of the cross-channel distribution of sediment.

\section{Polychlorinated Biphenyls and Polycyclic Aromatic Hydrocarbons in Suspended Sediment and Bed Sediment}

A summary of average tPCB, TEQ due to PCBs, tPAH (including both nonalkylated and alkylated compounds), and TEQ due to PAHs is presented in table 24. The low-flow samples from Hickey Run may be anomalous in that they contained much higher tPCB and tPAH concentrations than the other streams; the samples collected on November 17, 2016, and July 27, 2017, contained tPCB concentrations of 230 and $1,900 \mu \mathrm{g} / \mathrm{kg}$ (table 7), respectively, and tPAH concentrations of 101,000 and $67,000 \mu \mathrm{g} / \mathrm{kg}$ (table 9), respectively. These are the highest $\mathrm{tPAH}$ concentrations measured during this study and are higher than concentrations reported from contaminated urban rivers such as the Elizabeth and Passaic Rivers in New Jersey (2,000-5,000 $\mu \mathrm{g} / \mathrm{kg}$ ) (Wilson and Bonin, 2008). One explanation is that bed sediment or a separate oil phase may have been captured. The SSCs in grab samples collected over the sampling period averaged $6 \mathrm{mg} / \mathrm{L}$, a reasonable value for low flow. However, the water level during the low-flow sampling was extremely low, and it is possible that either bed sediment or floating oil was suctioned into the sampling line. Oil sheens were observed in Hickey Run throughout the study. Both low-flow samples (November 2016 and July 2017) contained high concentrations of PCBs and PAHs; therefore, it is possible that concentrations are indeed high during low flow. However, because the elevated concentrations of PCBs, PAHs, and pesticides in these two samples are suspect, the July 2017 sample was removed from the dataset before the average concentrations were calculated, but the November 2016 sample was retained to allow for the possibility that the elevated concentrations accurately represent the composition of the low-flow samples.

Average tPCB concentrations (table 24) ranged from $6.6 \mu \mathrm{g} / \mathrm{kg}$ (in NWB) to $130 \mu \mathrm{g} / \mathrm{kg}$ (in LBDC). Concentrations in low-flow samples (averaged when two samples were available) were higher than those in the stormflow samples by factors ranging from 2 to 9 . The maximum tPCB concentration found in this study was in sediment from LBDC $(130 \mu \mathrm{g} / \mathrm{kg})$, followed by Hickey Run $(69 \mu \mathrm{g} / \mathrm{kg})$ and Watts Branch
(44 $\mu \mathrm{g} / \mathrm{kg})$. Concentrations of tPCB in NEB and NWB were an order of magnitude lower -5.9 and $6.6 \mu \mathrm{g} / \mathrm{kg}$, respectively. The highest $\mathrm{PCB}$ concentration in the ungaged streams was found in Nash Run, where the tPCB concentration averaged $65 \mu \mathrm{g} / \mathrm{kg}$; concentrations in the other ungaged streams were much lower-Pope Branch, $1.5 \mu \mathrm{g} / \mathrm{kg}$; Fort DuPont Creek, $1.2 \mu \mathrm{g} / \mathrm{kg}$; and Fort Stanton Creek, $4.3 \mu \mathrm{g} / \mathrm{kg}$.

As noted previously, the average tPCB concentration in low-flow samples exceeded the average concentration in the storm samples by a factor ranging from 2.3 (in NEB) to 8.4 (in LBDC). For example, in NWB, the average concentration was $16 \mu \mathrm{g} / \mathrm{kg}$ in the two low-flow samples but only $2.1 \mu \mathrm{g} / \mathrm{kg}$ in the storm samples. In LBDC, the average concentration was $380 \mu \mathrm{g} / \mathrm{kg}$ in the low-flow samples but only $45 \mu \mathrm{g} / \mathrm{kg}$ in the storm samples. The average tPCB concentration in storm and low-flow samples tended to be more similar in the other ungaged streams than in Nash Run. For example, the average concentrations in low-flow and stormflow samples from Fort DuPont Creek were 1.2 and $1.9 \mu \mathrm{g} / \mathrm{kg}$, respectively.

In this study, sediment samples were collected during low flow because it is during low flow that only the finest particle-size materials are transported; these particles typically have a high organic carbon content and, therefore, elevated concentrations of the hydrophobic COCs, which is consistent with the observation that concentrations are higher during low flow than during stormflow. During stormflow, coarser, "cleaner" materials become entrained and dilute the finer materials in suspension, resulting in elevated concentrations in the sediment phase. As discussed farther on in this report, despite the large differences in concentrations of COCs between sediment samples collected during low flow and stormflow, the yearly loadings of COCs are dominated by those transported during storms.

Average concentrations of tPCBs in the bed sediment were compared with the concentrations in suspended sediment collected during stormflow and low flow. In LBDC and Watts Branch, the average $\mathrm{PCB}$ concentration in bed sediment was intermediate between the stormflow and low-flow suspendedsediment averages, whereas in the other gaged tributaries the average concentrations in bed sediment were less than the stormflow suspended-sediment averages. In Pope Branch, Fort DuPont Creek, and Fort Stanton Creek, the tPCB content in the bed sediment was nearly the same as the tPCB content in the suspended sediment (average of the concentrations in one storm and one low-flow sample). In Nash Run, however, the $\mathrm{APCB}$ content in the suspended sediment was nearly 40 times the $\mathrm{PCB}$ concentration in the bed sediment. Additional sampling and analysis would help to confirm this finding. For the other ungaged tributaries, however, these data indicate that newly deposited bed sediment has a tPCB content very nearly equal to that of the suspended sediment transported during storms.

Absolute concentrations of contaminants in suspended sediment can be affected by many processes, including dilution by "cleaner" sediment introduced under certain 
Table 24. Summary statistics of polychlorinated biphenyl and polycyclic aromatic hydrocarbon concentrations in tributaries to the Anacostia River.

$[\mu \mathrm{g} / \mathrm{kg}$, micrograms per kilogram; TEQ, toxic equivalency; LMW, low molecular weight; HMW, high molecular weight; PCB, polychlorinated biphenyl; PAH, polycyclic aromatic hydrocarbon; F, fluoranthene; PY, pyrene; PH, phenanthrene; A, anthracene; --, not applicable]

\begin{tabular}{|c|c|c|c|c|c|c|c|c|c|c|}
\hline \multirow[b]{2}{*}{ Statistic } & \multicolumn{6}{|c|}{ Suspended sediment } & \multicolumn{4}{|c|}{ Bed sediment } \\
\hline & $\begin{array}{l}\text { Average } \\
\text { of storm } \\
\text { samples }\end{array}$ & $\begin{array}{c}\text { Average of } \\
\text { low-flow } \\
\text { samples }\end{array}$ & $\begin{array}{l}\text { Average of } \\
\text { all samples }\end{array}$ & $\begin{array}{l}\text { Maximum } \\
\text { value }\end{array}$ & $\begin{array}{l}\text { Minimum } \\
\text { value }\end{array}$ & $\begin{array}{l}\text { Standard } \\
\text { deviation }\end{array}$ & Average & $\begin{array}{l}\text { Maximum } \\
\text { value }\end{array}$ & $\begin{array}{l}\text { Minimum } \\
\text { value }\end{array}$ & $\begin{array}{l}\text { Standard } \\
\text { deviation }\end{array}$ \\
\hline & \multicolumn{10}{|c|}{ Northeast Branch } \\
\hline Number of samples averaged & 5 & 2 & 7 & -- & -- & -- & 3 & -- & -- & -- \\
\hline Average number of PCB congeners detected & 79 & 86 & 81 & 104 & 60 & -- & 90 & 96 & 85 & -- \\
\hline Total PCB $(\mu \mathrm{g} / \mathrm{kg})$ & 4.2 & 9.8 & 5.9 & 19 & 1.4 & 6.0 & 2.3 & 4.5 & 0.90 & 1.9 \\
\hline PCB TEQ $(\mu \mathrm{g} / \mathrm{kg})$ & $2.9 \times 10^{-5}$ & $4.1 \times 10^{-4}$ & $2.5 \times 10^{-5}$ & $1.2 \times 10^{-4}$ & $2.5 \times 10^{-6}$ & $4.1 \times 10^{-5}$ & $9.8 \times 10^{-5}$ & $2.9 \times 10^{-4}$ & $1.6 \times 10^{-6}$ & $1.7 \times 10^{-4}$ \\
\hline Number of PAH compounds detected & 31 & 26 & 30 & 35 & 23 & -- & 30 & 31 & 29 & -- \\
\hline Total PAH $(\mu \mathrm{g} / \mathrm{kg})$ & 2,100 & 1,500 & 2,000 & 3,200 & 380 & 1,100 & 18,000 & 52,000 & 390 & 29,000 \\
\hline Sum nonalkylated PAH $(\mu \mathrm{g} / \mathrm{kg})$ & 1,700 & 1,100 & 1,600 & 2,700 & 280 & 870 & 16,000 & 47,000 & 230 & 26,000 \\
\hline Sum HMW PAH $(\mu \mathrm{g} / \mathrm{kg})$ & 1,600 & 1,100 & 1,400 & 2,400 & 260 & 800 & 15,000 & 43,000 & 210 & 24,000 \\
\hline Sum LMW PAH $(\mu \mathrm{g} / \mathrm{kg})$ & 160 & 79 & 140 & 240 & 23 & 74 & 1,200 & 3,500 & 15 & 2,000 \\
\hline Sum alkylated PAH ( $\mu \mathrm{g} / \mathrm{kg})$ & 410 & 370 & 360 & 670 & 63 & 240 & 2,100 & 5,300 & 160 & 2,800 \\
\hline Alkylated/nonalkylated concentration ratio & 24 & 28 & 25 & 38 & 18 & 7.7 & 47 & 72 & 11 & 31 \\
\hline Ratio of F/PY & 1.3 & 1.1 & 1.2 & 19 & 1.2 & 9.0 & 0.81 & 1.3 & 0.11 & 0.63 \\
\hline Ratio of $\mathrm{PH} / \mathrm{A}$ & 30 & 6.8 & 21 & 15 & 10 & 2.2 & 5.3 & 9.2 & 1.2 & 4.0 \\
\hline PAH TEQ $(\mu \mathrm{g} / \mathrm{kg})$ & 170 & 120 & 140 & 240 & 25 & 81 & 1,700 & 5,200 & 24 & 3,000 \\
\hline
\end{tabular}


Table 24. Summary statistics of polychlorinated biphenyl and polycyclic aromatic hydrocarbon concentrations in tributaries to the Anacostia River.-Continued

$[\mu \mathrm{g} / \mathrm{kg}$, micrograms per kilogram; TEQ, toxic equivalency; LMW, low molecular weight; HMW, high molecular weight; PCB, polychlorinated biphenyl; PAH, polycyclic aromatic hydrocarbon; F, fluoranthene; PY, pyrene; PH, phenanthrene; A, anthracene; --, not applicable]

\begin{tabular}{|c|c|c|c|c|c|c|c|c|c|c|}
\hline \multirow[b]{2}{*}{ Statistic } & \multicolumn{6}{|c|}{ Suspended sediment } & \multicolumn{4}{|c|}{ Bed sediment } \\
\hline & $\begin{array}{l}\text { Average } \\
\text { of storm } \\
\text { samples }\end{array}$ & $\begin{array}{l}\text { Average of } \\
\text { low-flow } \\
\text { samples }\end{array}$ & $\begin{array}{l}\text { Average of } \\
\text { all samples }\end{array}$ & $\begin{array}{l}\text { Maximum } \\
\text { value }\end{array}$ & $\begin{array}{l}\text { Minimum } \\
\text { value }\end{array}$ & $\begin{array}{l}\text { Standard } \\
\text { deviation }\end{array}$ & Average & $\begin{array}{l}\text { Maximum } \\
\text { value }\end{array}$ & $\begin{array}{l}\text { Minimum } \\
\text { value }\end{array}$ & $\begin{array}{l}\text { Standard } \\
\text { deviation }\end{array}$ \\
\hline & \multicolumn{10}{|c|}{ Northwest Branch } \\
\hline Number of samples averaged & 5 & 2 & 7 & -- & -- & -- & -- & -- & -- & -- \\
\hline Average number of PCB congeners detected & 79 & 89 & 80 & 111 & 62 & -- & 89 & 98 & 78 & -- \\
\hline Total PCB $(\mu \mathrm{g} / \mathrm{kg})$ & 2.1 & 16 & 6.6 & 20 & 1.2 & 7.3 & 1.7 & 2.0 & 1.4 & 0.31 \\
\hline PCB TEQ $(\mu \mathrm{g} / \mathrm{kg})$ & $1.4 \times 10^{-5}$ & $3.6 \times 10^{-5}$ & $2.0 \times 10^{-5}$ & $5.0 \times 10^{-5}$ & $2.1 \times 10^{-6}$ & $1.9 \times 10^{-5}$ & $2.4 \times 10^{-5}$ & $6.5 \times 10^{-5}$ & $2.7 \times 10^{-5}$ & $3.6 \times 10^{-5}$ \\
\hline Number of PAH compounds detected & 33 & 30 & 32 & 35 & 25 & -- & 31 & 31 & 30 & -- \\
\hline Total PAH $(\mu \mathrm{g} / \mathrm{kg})$ & 1,900 & 7,000 & 3,300 & 7,700 & 1,200 & 2,600 & 2,500 & 3,600 & 1,200 & 1,200 \\
\hline Sum nonalkylated PAH $(\mu \mathrm{g} / \mathrm{kg})$ & 1,400 & 5,000 & 2,400 & 5,900 & 860 & 1,900 & 2,100 & 2,900 & 1,000 & 970 \\
\hline Sum HMW PAH $(\mu \mathrm{g} / \mathrm{kg})$ & 1,200 & 4,600 & 2,200 & 5,500 & 740 & 1,800 & 1,900 & 2,700 & 970 & 880 \\
\hline Sum LMW PAH ( $\mu \mathrm{g} / \mathrm{kg})$ & 160 & 320 & 210 & 380 & 100 & 91 & 150 & 220 & 55 & 87 \\
\hline Sum alkylated PAH $(\mu \mathrm{g} / \mathrm{kg})$ & 490 & 2,000 & 930 & 2,300 & 270 & 780 & 440 & 700 & 200 & 250 \\
\hline Alkylated/nonalkylated concentration ratio & 37 & 43 & 38 & 57 & 26 & 11 & 21 & 24 & 19 & 2.7 \\
\hline Ratio of F/PY & 2.3 & 1.2 & 1.4 & 23 & 1.0 & 8.7 & 1.2 & 1.3 & 1.2 & 0.04 \\
\hline Ratio of PH/A & 8.8 & 8.8 & 8.8 & 11 & 6.5 & 1.5 & 8.7 & 11 & 6.1 & 2.3 \\
\hline PAH TEQ $(\mu \mathrm{g} / \mathrm{kg})$ & 140 & 490 & 240 & 610 & 90 & 190 & 240 & 330 & 130 & 100 \\
\hline
\end{tabular}


Table 24. Summary statistics of polychlorinated biphenyl and polycyclic aromatic hydrocarbon concentrations in tributaries to the Anacostia River.-Continued

$[\mu \mathrm{g} / \mathrm{kg}$, micrograms per kilogram; TEQ, toxic equivalency; LMW, low molecular weight; HMW, high molecular weight; PCB, polychlorinated biphenyl; PAH, polycyclic aromatic hydrocarbon; F, fluoranthene; $\mathrm{PY}$, pyrene; $\mathrm{PH}$, phenanthrene; A, anthracene; --, not applicable]

\begin{tabular}{|c|c|c|c|c|c|c|c|c|c|c|}
\hline \multirow[b]{2}{*}{ Statistic } & \multicolumn{6}{|c|}{ Suspended sediment } & \multicolumn{4}{|c|}{ Bed sediment } \\
\hline & $\begin{array}{l}\text { Average } \\
\text { of storm } \\
\text { samples }\end{array}$ & $\begin{array}{l}\text { Average of } \\
\text { low-flow } \\
\text { samples }\end{array}$ & $\begin{array}{l}\text { Average of } \\
\text { all samples }\end{array}$ & $\begin{array}{l}\text { Maximum } \\
\text { value }\end{array}$ & $\begin{array}{l}\text { Minimum } \\
\text { value }\end{array}$ & $\begin{array}{l}\text { Standard } \\
\text { deviation }\end{array}$ & Average & $\begin{array}{l}\text { Maximum } \\
\text { value }\end{array}$ & $\begin{array}{l}\text { Minimum } \\
\text { value }\end{array}$ & $\begin{array}{l}\text { Standard } \\
\text { deviation }\end{array}$ \\
\hline & \multicolumn{10}{|c|}{ Beaverdam Creek } \\
\hline Number of samples averaged & 6 & 2 & 8 & -- & -- & -- & 5 & -- & -- & -- \\
\hline Average number of $\mathrm{PCB}$ congeners detected & 111 & 124 & 114 & 129 & 96 & -- & 121 & 128 & 107 & -- \\
\hline Total PCB $(\mu \mathrm{g} / \mathrm{kg})$ & 45 & 380 & 130 & 470 & 5.4 & 170 & 59 & 78 & 41 & 14 \\
\hline PCB TEQ $(\mu \mathrm{g} / \mathrm{kg})$ & $3.4 \times 10^{-4}$ & $6.0 \times 10^{-4}$ & $4.1 \times 10^{-4}$ & $1.9 \times 10^{-3}$ & $8.9 \times 10^{-6}$ & $6.4 \times 10^{-4}$ & $5.1 \times 10^{-4}$ & $1.4 \times 10^{-3}$ & $6.7 \times 10^{-5}$ & $5.9 \times 10^{-4}$ \\
\hline Number of PAH compounds detected & 34 & 35 & 34 & 35 & 29 & 2 & 32 & 35 & 30 & -- \\
\hline Total PAH $(\mu \mathrm{g} / \mathrm{kg})$ & 1,600 & 3,900 & 2,200 & 5,600 & 320 & 1,700 & 2,600 & 6,200 & 680 & 2,200 \\
\hline Sum nonalkylated PAH $(\mu \mathrm{g} / \mathrm{kg})$ & 1,300 & 2,700 & 1,700 & 3,900 & 260 & 1,200 & 2,200 & 5,400 & 550 & 1,900 \\
\hline Sum HMW PAH ( $\mu \mathrm{g} / \mathrm{kg})$ & 1,200 & 2,500 & 1,500 & 3,800 & 230 & 1,200 & 2,000 & 4,800 & 520 & 1,700 \\
\hline Sum LMW PAH ( $\mu \mathrm{g} / \mathrm{kg})$ & 120 & 210 & 140 & 290 & 30 & 74 & 220 & 680 & 35 & 260 \\
\hline Sum alkylated PAH $(\mu \mathrm{g} / \mathrm{kg})$ & 290 & 1,100 & 490 & 1,400 & 60 & 450 & 390 & 810 & 130 & 250 \\
\hline Alkylated/nonalkylated concentration ratio & 22 & 46 & 28 & 56 & 20 & 13 & 21 & 30 & 15 & 6.1 \\
\hline Ratio of F/PY & 1.1 & 0.93 & 0.98 & 1.2 & 0.85 & 0.10 & 1.1 & 1.2 & 1.1 & 0.07 \\
\hline Ratio of PH/A & 8.8 & 5.5 & 6.4 & 10 & 3.9 & 2.2 & 7.9 & 13 & 3.8 & 3.2 \\
\hline PAH TEQ $(\mu \mathrm{g} / \mathrm{kg})$ & 130 & 280 & 170 & 440 & 22 & 140 & 240 & 570 & 66 & 200 \\
\hline
\end{tabular}


Table 24. Summary statistics of polychlorinated biphenyl and polycyclic aromatic hydrocarbon concentrations in tributaries to the Anacostia River.-Continued

[ $\mu \mathrm{g} / \mathrm{kg}$, micrograms per kilogram; TEQ, toxic equivalency; LMW, low molecular weight; HMW, high molecular weight; PCB, polychlorinated biphenyl; PAH, polycyclic aromatic hydrocarbon; F, fluoranthene; PY, pyrene; PH, phenanthrene; A, anthracene; --, not applicable]

\begin{tabular}{|c|c|c|c|c|c|c|c|c|c|c|}
\hline \multirow[b]{2}{*}{ Statistic } & \multicolumn{6}{|c|}{ Suspended sediment } & \multicolumn{4}{|c|}{ Bed sediment } \\
\hline & $\begin{array}{l}\text { Average } \\
\text { of storm } \\
\text { samples }\end{array}$ & $\begin{array}{l}\text { Average of } \\
\text { low-flow } \\
\text { samples }\end{array}$ & $\begin{array}{l}\text { Average of } \\
\text { all samples }\end{array}$ & $\begin{array}{l}\text { Maximum } \\
\text { value }\end{array}$ & $\begin{array}{l}\text { Minimum } \\
\text { value }\end{array}$ & $\begin{array}{l}\text { Standard } \\
\text { deviation }\end{array}$ & Average & $\begin{array}{l}\text { Maximum } \\
\text { value }\end{array}$ & $\begin{array}{l}\text { Minimum } \\
\text { value }\end{array}$ & $\begin{array}{l}\text { Standard } \\
\text { deviation }\end{array}$ \\
\hline & \multicolumn{10}{|c|}{ Watts Branch } \\
\hline Number of samples averaged & 5 & 1 & 6 & -- & -- & -- & 3 & -- & -- & -- \\
\hline Average number of PCB congeners detected & 86 & 124 & 94 & 124 & 57 & -- & 114 & 117 & 113 & -- \\
\hline Total PCB $(\mu \mathrm{g} / \mathrm{kg})$ & 13 & 78 & 44 & 140 & 4.8 & 52 & 26 & 37 & 15 & 11 \\
\hline PCB TEQ $(\mu \mathrm{g} / \mathrm{kg})$ & $1.4 \times 10^{-4}$ & $2.0 \times 10^{-4}$ & $1.7 \times 10^{-4}$ & $3.8 \times 10^{-4}$ & $1.2 \times 10^{-5}$ & $1.7 \times 10^{-4}$ & $6.9 \times 10^{-5}$ & $9.5 \times 10^{-5}$ & $3.8 \times 10^{-5}$ & $2.8 \times 10^{-5}$ \\
\hline Number of PAH compounds detected & 34 & 33 & 34 & 35 & 33 & -- & 32 & 33 & 31 & -- \\
\hline Total PAH $(\mu \mathrm{g} / \mathrm{kg})$ & 1,900 & 5,100 & 2,400 & 5,100 & 1,000 & 1,700 & 3,300 & 4,800 & 1,600 & 1,600 \\
\hline Sum nonalkylated PAH $(\mu \mathrm{g} / \mathrm{kg})$ & 1,400 & 3,700 & 1,800 & 3,700 & 730 & 1,300 & 2,600 & 3,700 & 1,200 & 1,200 \\
\hline Sum HMW PAH ( $\mu \mathrm{g} / \mathrm{kg})$ & 1,300 & 3,200 & 1,600 & 3,200 & 670 & 1,100 & 2,300 & 3,300 & 1,100 & 1,100 \\
\hline Sum LMW PAH ( $\mu \mathrm{g} / \mathrm{kg})$ & 120 & 420 & 170 & 420 & 57 & 140 & 240 & 350 & 110 & 120 \\
\hline Sum alkylated PAH ( $\mu \mathrm{g} / \mathrm{kg})$ & 500 & 1,400 & 650 & 1,400 & 290 & 450 & 690 & 1,100 & 3,700 & 360 \\
\hline Alkylated/nonalkylated concentration ratio & 37 & 39 & 38 & 51 & 31 & 8 & 27 & 30 & 22 & 4.5 \\
\hline Ratio of F/PY & 1.1 & 1.0 & 1.1 & 1.2 & 1.0 & 0.06 & 1.1 & 1.1 & 1.0 & 0.09 \\
\hline Ratio of $\mathrm{PH} / \mathrm{A}$ & 6.6 & 4.6 & 5.4 & 8.7 & 4.6 & 1.4 & 6.0 & 6.1 & 5.9 & 0.14 \\
\hline PAH TEQ $(\mu \mathrm{g} / \mathrm{kg})$ & 140 & 360 & 180 & 350 & 74 & 130 & 300 & 430 & 150 & 140 \\
\hline
\end{tabular}


Table 24. Summary statistics of polychlorinated biphenyl and polycyclic aromatic hydrocarbon concentrations in tributaries to the Anacostia River.-Continued

$[\mu \mathrm{g} / \mathrm{kg}$, micrograms per kilogram; TEQ, toxic equivalency; LMW, low molecular weight; HMW, high molecular weight; PCB, polychlorinated biphenyl; PAH, polycyclic aromatic hydrocarbon; F, fluoranthene; PY, pyrene; PH, phenanthrene; A, anthracene; --, not applicable]

\begin{tabular}{|c|c|c|c|c|c|c|c|c|c|c|}
\hline \multirow[b]{2}{*}{ Statistic } & \multicolumn{6}{|c|}{ Suspended sediment } & \multicolumn{4}{|c|}{ Bed sediment } \\
\hline & $\begin{array}{l}\text { Average } \\
\text { of storm } \\
\text { samples }\end{array}$ & $\begin{array}{c}\text { Average of } \\
\text { low-flow } \\
\text { samples }\end{array}$ & $\begin{array}{l}\text { Average of } \\
\text { all samples }\end{array}$ & $\begin{array}{l}\text { Maximum } \\
\text { value }\end{array}$ & $\begin{array}{l}\text { Minimum } \\
\text { value }\end{array}$ & $\begin{array}{l}\text { Standard } \\
\text { deviation }\end{array}$ & Average & $\begin{array}{l}\text { Maximum } \\
\text { value }\end{array}$ & $\begin{array}{l}\text { Minimum } \\
\text { value }\end{array}$ & $\begin{array}{l}\text { Standard } \\
\text { deviation }\end{array}$ \\
\hline & \multicolumn{10}{|c|}{ Hickey Run ${ }^{1}$} \\
\hline Number of samples averaged & 4 & 1 & 5 & -- & -- & -- & 5 & -- & -- & -- \\
\hline Average number of $\mathrm{PCB}$ congeners detected & 83 & 94 & 85 & 97 & 68 & -- & 103 & 121 & 87 & -- \\
\hline Total PCB $(\mu \mathrm{g} / \mathrm{kg})$ & 28 & 230 & 69 & 230 & 11 & 92 & 21 & 35 & 14 & 8.6 \\
\hline PCB TEQ $(\mu \mathrm{g} / \mathrm{kg})$ & $3.8 \times 10^{-4}$ & $7.30 \times 10^{-4}$ & $4.51 \times 10^{-4}$ & $1.3 \times 10^{-3}$ & $7.3 \times 10^{-6}$ & $5.8 \times 10^{-4}$ & $1.8 \times 10^{-4}$ & $7.0 \times 10^{-4}$ & $2.5 \times 10^{-5}$ & $2.9 \times 10^{-4}$ \\
\hline Number of PAH compounds detected & 35 & 34 & 35 & 35 & 34 & -- & 34 & 35 & 32 & -- \\
\hline Total PAH $(\mu \mathrm{g} / \mathrm{kg})$ & 6,300 & 66,000 & 18,000 & 66,000 & 4,500 & 27,000 & 12,000 & 18,000 & 4,000 & 6,000 \\
\hline Sum nonalkylated PAH $(\mu \mathrm{g} / \mathrm{kg})$ & 3,900 & 11,000 & 5,400 & 11,000 & 3,200 & 3,400 & 8,600 & 14,000 & 2,200 & 4,600 \\
\hline Sum HMW PAH $(\mu \mathrm{g} / \mathrm{kg})$ & 3,400 & 9,900 & 4,700 & 9,900 & 2,800 & 3,000 & 7,500 & 12,000 & 2,000 & 4,000 \\
\hline Sum LMW PAH $(\mu \mathrm{g} / \mathrm{kg})$ & 480 & 1,100 & 610 & 11,000 & 310 & 320 & 1,100 & 1,700 & 200 & 590 \\
\hline Sum alkylated PAH $(\mu \mathrm{g} / \mathrm{kg})$ & 2,400 & 55,000 & 13,000 & 55,000 & 1,300 & 24,000 & 3,700 & 7,500 & 1,900 & 2,300 \\
\hline Alkylated/nonalkylated concentration ratio & 62 & 35 & 56 & 86 & 35 & 20 & 51 & 85 & 23 & 27 \\
\hline Ratio of F/PY & 0.92 & 0.27 & 0.33 & 0.98 & 0.63 & 0.14 & 1.0 & 1.4 & 0.69 & 0.29 \\
\hline Ratio of $\mathrm{PH} / \mathrm{A}$ & 8.3 & 26 & 17 & 8.8 & 3.1 & 2.4 & 8.9 & 18 & 4.1 & 5.1 \\
\hline PAH TEQ $(\mu \mathrm{g} / \mathrm{kg})$ & 390 & 710 & 450 & 710 & 310 & 180 & 810 & 1300 & 240 & 410 \\
\hline
\end{tabular}


Table 24. Summary statistics of polychlorinated biphenyl and polycyclic aromatic hydrocarbon concentrations in tributaries to the Anacostia River.-Continued

$[\mu \mathrm{g} / \mathrm{kg}$, micrograms per kilogram; TEQ, toxic equivalency; LMW, low molecular weight; HMW, high molecular weight; PCB, polychlorinated biphenyl; PAH, polycyclic aromatic hydrocarbon; F, fluoranthene; PY, pyrene; PH, phenanthrene; A, anthracene; --, not applicable]

\begin{tabular}{|c|c|c|c|c|c|c|c|c|c|c|}
\hline \multirow[b]{2}{*}{ Statistic } & \multicolumn{4}{|c|}{ Suspended sediment } & \multirow[b]{2}{*}{$\begin{array}{c}\text { Bed } \\
\text { sediment }\end{array}$} & \multicolumn{4}{|c|}{ Suspended sediment } & \multirow[b]{2}{*}{$\begin{array}{c}\text { Bed } \\
\text { sediment }\end{array}$} \\
\hline & $\begin{array}{l}\text { Average } \\
\text { of storm } \\
\text { samples }\end{array}$ & $\begin{array}{l}\text { Maximum } \\
\text { value }\end{array}$ & $\begin{array}{l}\text { Minimum } \\
\text { value }\end{array}$ & $\begin{array}{l}\text { Standard } \\
\text { deviation }\end{array}$ & & $\begin{array}{l}\text { Average } \\
\text { of storm } \\
\text { samples }\end{array}$ & $\begin{array}{l}\text { Maximum } \\
\text { value }\end{array}$ & $\begin{array}{c}\text { Minimum } \\
\text { value }\end{array}$ & $\begin{array}{l}\text { Standard } \\
\text { deviation }\end{array}$ & \\
\hline & \multicolumn{5}{|c|}{ Nash Run } & \multicolumn{5}{|c|}{ Pope Branch } \\
\hline Number of samples averaged & 5 & -- & -- & -- & 3 & 2 & -- & -- & -- & 1 \\
\hline Average number of $\mathrm{PCB}$ congeners detected & 86 & 124 & 57 & -- & 114 & 70 & 91 & 49 & -- & 98 \\
\hline Total PCB $(\mu \mathrm{g} / \mathrm{kg})$ & 13 & 140 & 4.8 & 52 & 26 & 1.5 & 2.1 & 0.92 & 0.83 & 1.3 \\
\hline PCB TEQ $(\mu \mathrm{g} / \mathrm{kg})$ & $1.4 \times 10^{-4}$ & $3.8 \times 10^{-4}$ & $1.2 \times 10^{-5}$ & $1.7 \times 10^{-4}$ & $6.9 \times 10^{-5}$ & $2.1 \times 10^{-4}$ & $3.8 \times 10^{-6}$ & $4.4 \times 10^{-7}$ & $2.4 \times 10^{-6}$ & $3.6 \times 10^{-5}$ \\
\hline Number of PAH compounds detected & 34 & 35 & 33 & -- & 32 & 13 & 14 & 12 & 1 & 32 \\
\hline Total PAH $(\mu \mathrm{g} / \mathrm{kg})$ & 1,900 & 5,100 & 1,000 & 1,700 & 3,300 & 630 & 760 & 500 & 190 & 280 \\
\hline Sum nonalkylated $\mathrm{PAH}(\mu \mathrm{g} / \mathrm{kg})$ & 1,400 & 3,700 & 730 & 1,300 & 2,600 & 370 & 570 & 180 & 270 & 210 \\
\hline Sum HMW PAH ( $\mu \mathrm{g} / \mathrm{kg})$ & 1,300 & 3,200 & 670 & 1,100 & 2,300 & 320 & 520 & 110 & 280 & 190 \\
\hline Sum LMW PAH $(\mu \mathrm{g} / \mathrm{kg})$ & 120 & 420 & 57 & 140 & 240 & 56 & 65 & 47 & 12 & 20 \\
\hline Sum alkylated PAH $(\mu \mathrm{g} / \mathrm{kg})$ & 500 & 1,400 & 290 & 450 & 690 & 260 & 320 & 200 & 85 & 71 \\
\hline Alkylated/nonalkylated concentration ratio & 37 & 51 & 31 & 8 & 27 & 24 & 34 & 12 & 16 & 34 \\
\hline Ratio of F/PY & 1.1 & 1.2 & 1.0 & 0.06 & 1.1 & 1.1 & 1.1 & 1.1 & 0.01 & 1.0 \\
\hline Ratio of PH/A & 6.6 & 8.7 & 4.6 & 1.4 & 6.0 & 6.8 & 6.9 & 6.4 & 0.36 & 4.8 \\
\hline PAH TEQ $(\mu \mathrm{g} / \mathrm{kg})$ & 140 & 350 & 74 & 130 & 300 & 34 & 57 & 11 & 33 & 26 \\
\hline
\end{tabular}


Table 24. Summary statistics of polychlorinated biphenyl and polycyclic aromatic hydrocarbon concentrations in tributaries to the Anacostia River.-Continued

$[\mu \mathrm{g} / \mathrm{kg}$, micrograms per kilogram; TEQ, toxic equivalency; LMW, low molecular weight; HMW, high molecular weight; PCB, polychlorinated biphenyl; PAH, polycyclic aromatic hydrocarbon; F, fluoranthene; PY, pyrene; PH, phenanthrene; A, anthracene; --, not applicable]

\begin{tabular}{|c|c|c|c|c|c|c|c|c|c|c|}
\hline \multirow[b]{2}{*}{ Statistic } & \multicolumn{4}{|c|}{ Suspended sediment } & \multirow[b]{2}{*}{$\begin{array}{c}\text { Bed } \\
\text { sediment }\end{array}$} & \multicolumn{4}{|c|}{ Suspended sediment } & \multirow[b]{2}{*}{$\begin{array}{c}\text { Bed } \\
\text { sediment }\end{array}$} \\
\hline & $\begin{array}{l}\text { Average } \\
\text { of storm } \\
\text { samples }\end{array}$ & $\begin{array}{l}\text { Maximum } \\
\text { value }\end{array}$ & $\begin{array}{l}\text { Minimum } \\
\text { value }\end{array}$ & $\begin{array}{l}\text { Standard } \\
\text { deviation }\end{array}$ & & $\begin{array}{l}\text { Average } \\
\text { of storm } \\
\text { samples }\end{array}$ & $\begin{array}{c}\text { Maximum } \\
\text { value }\end{array}$ & $\begin{array}{c}\text { Minimum } \\
\text { value }\end{array}$ & $\begin{array}{l}\text { Standard } \\
\text { deviation }\end{array}$ & \\
\hline & \multicolumn{5}{|c|}{ Fort DuPont Creek } & \multicolumn{5}{|c|}{ Fort Stanton Creek } \\
\hline Number of samples averaged & 2 & -- & -- & -- & 1 & 2 & -- & -- & -- & 1 \\
\hline Average number of PCB congeners detected & 67 & 89 & 44 & 32 & 81 & 76 & 82 & 70 & -- & 89 \\
\hline Total PCB $(\mu \mathrm{g} / \mathrm{kg})$ & 1.2 & 1.9 & 0.50 & 0.98 & 1.2 & 5.5 & 9.7 & 1.3 & 5.9 & 2.0 \\
\hline PCB TEQ $(\mu \mathrm{g} / \mathrm{kg})$ & $3.7 \times 10^{-5}$ & $7.4 \times 10^{-5}$ & $2.0 \times 10^{-7}$ & $5.2 \times 10^{-5}$ & $1.2 \times 10^{-4}$ & $2.8 \times 10^{-5}$ & $4.2 \times 10^{-5}$ & $1.4 \times 10^{-5}$ & $2.0 \times 10^{-5}$ & $3.8 \times 10^{-6}$ \\
\hline Number of PAH compounds detected & 35 & 35 & 34 & -- & 35 & 28 & 31 & 24 & -- & 31 \\
\hline Total PAH ( $\mu \mathrm{g} / \mathrm{kg})$ & 350 & 380 & 310 & 47 & 4,600 & 710 & 830 & 590 & 170 & 380 \\
\hline Sum nonalkylated PAH ( $\mu \mathrm{g} / \mathrm{kg})$ & 270 & 300 & 250 & 34 & 340 & 550 & 630 & 470 & 110 & 300 \\
\hline Sum HMW PAH ( $\mu \mathrm{g} / \mathrm{kg})$ & 230 & 260 & 210 & 33 & 300 & 420 & 490 & 350 & 97 & 270 \\
\hline Sum LMW PAH $(\mu \mathrm{g} / \mathrm{kg})$ & 33 & 34 & 33 & 0.85 & 31 & 50 & 55 & 46 & 6.9 & 30 \\
\hline Sum alkylated PAH $(\mu \mathrm{g} / \mathrm{kg})$ & 75 & 84 & 66 & 13 & 120 & 230 & 280 & 180 & 71 & 82 \\
\hline Alkylated/nonalkylated concentration ratio & 27 & 28 & 27 & 1.2 & 35 & 27 & 40 & 15 & 17 & 28 \\
\hline Ratio of F/PY & 1.1 & 1.1 & 1.1 & 0 & 1.1 & 0.94 & 1.0 & 0.88 & 0.09 & 1.1 \\
\hline Ratio of $\mathrm{PH} / \mathrm{A}$ & 5.8 & 5.8 & 5.8 & 0 & 4.6 & 5.2 & 6.7 & 4.9 & 1.3 & 4.8 \\
\hline PAH TEQ $(\mu \mathrm{g} / \mathrm{kg})$ & 27 & 30 & 23 & 4.9 & 37 & 46 & 57 & 36 & 15 & 34 \\
\hline
\end{tabular}


hydrologic conditions. For example, most "normal-range" storm events act only to suspend bed sediment that has been well mixed as it progressively migrates downstream. During extreme events, larger grained coarse sand and gravel are mobilized by streambank erosion. These coarser materials typically are less able to sequester contaminants or have not been affected by historical contamination. Their introduction results in "solid material dilution" (addition of sediment carrying contaminants). Moreover, contaminant concentrations associated with sediment introduced by street runoff may differ greatly by season. Finally, during low flow the particulate carbon carried by streamwater may vary in both quantity and quality during the year-for example, tree-leaf debris enters the streams during fall. All of these factors affect the absolute concentrations of contaminants measured in suspended-sediment samples collected from tributaries. Before concentrations in multiple samples collected over a year are averaged, therefore, the similarities and differences among the samples from each tributary must be considered.

The PCB congener profiles of the different streams were evaluated to demonstrate similarities and differences among the streams. Many techniques have been used to make such comparisons in earlier studies (Wilson and Bonin, 2007, 2008). One common method is to construct a histogram of the weight percentage of PCBs in each homolog group. While allowing the PCB compositions in different types of sediment to be compared, the large differences in the number and weights of the congeners composing each homolog group force the weight-percent to plot in a bell-shaped pattern centered around the hepta- and hexa-homolog groups. These homologs were the most abundant in the manufactured aroclors that were the ultimate source of the PCBs (Erickson, 1997). As a result, weight percentages commonly provide little insight into differentiating samples.

Because the goal of this study was to estimate loadings, only a cursory evaluation of the PCB congener chemistry was made by using the molar percentage of the congeners. Mole percentages can be thought of as representing the number of molecules of each congener per unit mass of sediment. Typically, little useful information is gained by including the congeners present in trace amounts (Wilson and Bonin, 2007, 2008); therefore, this evaluation is limited to congeners present at concentrations greater than 1 mole percent.

Molar percentages of averaged concentrations in suspended-sediment and bed-sediment samples are plotted in figure 9. Low-flow and storm sediment samples from NEB, NWB, LBDC, and Watts Branch had similar congener compositions. Sediment in low-flow samples from Hickey Run, however, contained much higher percentages of congeners from PCB-45 to PCB-154, whereas sediment in storm samples from this stream contained higher percentages of PCB congeners numbered 155 and greater.
A test statistic equivalent to the RMSE of regression analysis provides a measure of the difference among sediment samples. The average difference among samples is defined as

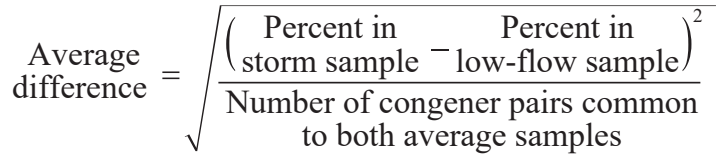

The average difference between low-flow and stormflow PCB concentrations is 0.04 percent for NEB, 0.05 percent for NWB, 0.03 percent for LBDC, 0.08 percent for Hickey Run, and 0.17 percent for Watts Branch. This result supports the validity of using an average concentration for PCBs to calculate loads. The similarity in ratios of concentrations of highmolecular-weight (HMW) to low-molecular-weight (LMW) compounds, the ratio of total alkylated to nonalkylated concentrations, and the ratios of the concentrations of fluoranthene to pyrene and phenanthrenes to anthracene in the dataset support the appropriateness of using averages for the PAH compounds as well.

The following observations can be made from graphs of molar distribution of PCBs in the sediments (fig. 9):

1. NEB (fig. 9A) and NWB (fig. 9B): PCB-11 was abundant in both streams, with molar percentages of 4 to 5 percent in samples from NEB and about 8 percent in those from NWB. PCB-11 was present in the bed-sediment samples at a similar percentage as well. The congeners present in the highest percentages were PCB-129/138/160/163, PCB147/149, PCB-154, and PCB-180 through PCB-193. Generally, the molar percentages were similar in the three types of samples from NEB. PCB-203 was present at more than 1.5 percent in the low-flow suspended sediment from NWB but was present only in trace percentages in the storm sediment and bed sediment. PCBs heavier than PCB-187 (from the hepta-homolog group) were present in trace amounts in aroclor 1254 but were present in higher percentages in aroclor 1260. Generally, PCB-84 through PCB-154 (pentaand hexa-homologs) made up the largest percentages in the low-flow and stormflow samples and in bed sediments.

2. LBDC (fig. 9C): The makeup of PCB congeners in samples from the LBDC differed from that in samples from the other tributaries, as high percentages of congeners from the mono- through tetra-homolog groups were present. Several of the di-chloro (PCB-4 to PCB-15) and tri-chloro (PCB-16 to $\mathrm{PCB}-39) \mathrm{PCBs}$ were present at percentages considerably greater than 1 percent in the low-flow, storm, and bedsediment samples. The percentage of PCB congeners more chlorinated than PCB-82 was nearly identical in low-flow, storm, and bed-sediment samples. 
3. Watts Branch (fig. 9D): The PCB congener profiles in samples from this stream are typical of those found in many studies (Wilson and Bonin, 2007, 2008; Erickson, 1997), consisting of mainly PCB-80 through PCB-154, the penta- and hexa-homolog groups. Similar distributions of congeners were found in the low-flow, storm, and bedsediment samples.

4. Hickey Run (fig. 9E): The congener profiles of all samples from Hickey Run were characterized by the presence of PCBs from all homolog groups. The profile for Hickey Run sediment samples is similar to that for samples from NEB and NWB, although the percentages for the PCBs congeners with numbers greater than 169 (the heptahomologs) were higher in Hickey Run samples, especially for PCB-180/193. Like samples from NEB and NWB, Hickey Run sediment samples also contained substantial percentages of PCBs in the octa-homolog group (PCB194 to PCB-295), which were not present (at concentrations $>1$ percent) in sediment samples from Watts Branch or LBDC. Storm-sediment samples generally contained greater percentages of $\mathrm{PCB}$ congeners with numbers greater than 170 (hepta-homologs and above).

5. PCB toxicity: Hickey Run sediment was found to contain the highest PCB toxicity $\left(4.5 \times 10^{-4} \mu \mathrm{g} / \mathrm{kg}\right.$ as TEQ), a value just slightly greater than those associated with the sediment from LBDC $\left(3.5 \times 10^{-4} \mu \mathrm{g} / \mathrm{kg}\right)$ and Watts Branch $\left(1.5 \times 10^{-4} \mu \mathrm{g} / \mathrm{kg}\right)$. Toxicity of sediment from NEB and NWB was an order of magnitude smaller, $2.5 \times 10^{-5}$ and $1.8 \times 10^{-5} \mu \mathrm{g} / \mathrm{kg}$, respectively.

6. Nash Run (fig. 9F): The congener profile of sediment from Nash Run generally resembles those of NEB (fig. 9A) and NWB (fig. 9B) sediment. Compared with Watts Branch sediment, Nash Run sediment contained higher percentages of PCBs lighter than PCB-42, including PCB-11. The profile for Nash Run sediment does not resemble the profile in sediment from LBDC (fig. 9C), although these basins adjoin one another.

7. Pope Branch (fig. 9G): The congener percentages in Pope Branch sediment were much higher than those in sediment from the other tributaries; the percentage of PCB-11 in storm sediment, for example, represents nearly 25 percent of the total congener makeup. However, the very high percentages result from the very low concentrations of all the PCBs, with tPCBs from 1 to $2 \mu \mathrm{g} / \mathrm{kg}$. All three sediment types contained a high percentage of PCB-11 and PCB-154.

\section{Fort DuPont (fig. 9H) and Fort Stanton (fig. 9I)}

Creeks: These tributaries have similar congener profiles, although absolute concentrations of all PCBs were very low, as they were in sediment from Pope Branch. Most notable in these profiles are the high percentages of PCBs 170 (hepta-homologs) through 203 (octa-homologs).
9. Other observations: The PCB congener present in the highest concentrations (and molar percentages) in all streams except Fort Stanton Creek was the coelution PCB-129/138/160/163, congeners from the hexa-homolog group. Two possible explanations for the dominance of this coelution exist. First, because this is a coelution of congeners, the total concentration reported may be the sum of up to four congeners rather than a single or pair of congeners, as is the case for many of the other PCBs. Second, PCB-129 and PCB-138 were present at very high concentrations (and molar percentages) in the parent aroclors 1254 and 1260, but not in the lighter aroclors. Although aroclors vary in their makeup, PCB-138 was reported to be present at concentrations of more than 72 percent by weight and PCB-129 was present at concentrations of about 1 percent in these two aroclors (Erickson, 1997; Kodananti and others, 2001). Because of the prevalence of these congeners in the more highly chlorinated aroclors, it is likely that the concentration reported for coelution 129/138/160/163 represents multiple congeners, at least one of which (PCB-138) was extremely abundant. Several other congeners dominated random sediment samples from the tributaries. Examples include PCB-153/168 in samples from NEB and Hickey Run; the coelution PCB-90/101/113 along with PCB-118, PCB147, and PCB-180 in samples from NWB; the coelution PCB-90/101/113 in samples from Watts Branch; PCB180/193 in samples from Hickey Run; and PCB-153/168 in samples from Pope Branch. In Fort Stanton Creek sediment, the PCB congers 180/193 and 147/149 were present at the highest concentrations in the LF and storm samples from this stream. These dominant congeners are from hexa- and penta-homolog groups, and, like PCBs 90, 101, 118,153 , and 149, were abundant in aroclors 1254 and 1260. The high concentrations of PCB-11 in samples from NEB and NWB are likely the result of the association of this congener with the manufacture of paint pigments, including the marking paint used on pavement.

Average tPAH concentrations, calculated as the sum of the nonalkylated and alkylated species, were $2,000 \mu \mathrm{g} / \mathrm{kg}$ in NEB; $3,300 \mu \mathrm{g} / \mathrm{kg}$ in NWB; $2,200 \mu \mathrm{g} / \mathrm{kg}$ in LBDC; $2,400 \mu \mathrm{g} / \mathrm{kg}$ in Watts Branch; and $18,000 \mu \mathrm{g} / \mathrm{kg}$ in Hickey Run. Because these totals include the alkylated forms, these concentrations cannot be compared with results of analyses from other studies, including the Phase I study (TetraTech, 2016), as the suite of compounds included in the tPAH values greatly affects the loadings of PAHs. Average totals for nonalkylated compounds were $1,600 \mu \mathrm{g} / \mathrm{kg}$ in NEB; $2,400 \mu \mathrm{g} / \mathrm{kg}$ in NWB; $1,700 \mu \mathrm{g} / \mathrm{kg}$ in LBDC; $1,800 \mu \mathrm{g} / \mathrm{kg}$ in Watts Branch; and $5,400 \mu \mathrm{g} / \mathrm{kg}$ in Hickey Run. With the exception of Hickey Run, the nonalkylated compounds represent approximately 80 percent of the $\mathrm{PAH}$ concentrations. The concentrations in NEB and NWB are within the range reported by Foster and others (2000) and Hwang and Foster (2008). 
Various suites of compounds have been shown to be useful as indicators of PAH sources. These include the ratio of concentrations of total nonalkylated species to total PAHs, and the ratios of concentrations of fluoranthene to pyrene, phenanthrene to anthracene, and methyl phenanthrene to phenanthrene (Steinhauer and Boehm, 1992). Although determining the source of the PAHs is not the purpose of this report, several ratios show the similarities and differences among the tributaries and are discussed below.

General observations regarding the PAH makeup of the storm and low-flow samples include-

1. NEB: Most (80 percent) of the $\mathrm{PAH}$ concentration is made up of the nonalkylated PAHs; of this total, 94 percent are the HMW compounds. The alkylated species compose 20 percent of the IPAH concentration. The average concentration ratio of total alkylated compounds to nonalkylated compounds is 25 .

2. NWB: Most (73 percent) of the tPAH concentration is made up of nonalkylated species, slightly less than that found in NEB. Of the total nonalkylated concentration, 92 percent are HMW compounds. Alkylated species make up 27 percent of the $\mathrm{PAH}$ concentration, and the alkylated/nonalkylated concentration ratio is 38 ; both values are higher than those found in NEB samples.

3. LBDC: Most (77 percent) of the tPAH concentration is made up of nonalkylated species, with 88 percent of the total concentration being HMW compounds. The alkylated species make up 22 percent of the tPAH concentration, and the ratio of total alkylated to nonalkylated concentrations is 28 , intermediate between the ratios in NEB and NWB.

4. Hickey Run: Only 30 percent of the tPAH concentration is made up of the nonalkylated PAH compounds, of which 87 percent are HMW compounds. The alkylated species dominate, representing 70 percent of the PAH concentration, and the sediment has an alkylated/nonalkylated concentration ratio of 56. Hickey Run sediment samples clearly differ in this way from those collected from the other streams but, as described previously, the low-flow sample may be anomalous and may have skewed these ratios. If only the storm samples are considered, the nonalkylated species compose 62 percent of the tPAH concentration, of which 87 percent are HMW compounds. Alkylated species represent 38 percent of the tPAH concentration. Ratios of alkylated to nonalkylated species demonstrate that the alkylated species account for a larger proportion of the $\mathrm{PAH}$ concentration than in the other streams.

5. Watts Branch: Most (75 percent) of the tPAH concentration is composed of the nonalkylated compounds, of which 89 percent are HMW compounds. Alkylated species compose 25 percent of the total, and the ratio of alkylated to nonalkylated compounds is 38 , the same as in NWB. The PAH makeup of sediment in this tributary is similar to that in NEB, NWB, and LBDC.
General observations regarding the PAHs in the ungaged streams include-

1. Nash Run: Most (76 percent) of the tPAH concentration is made up of the nonalkylated compounds, of which more than 90 percent are HMW compounds. Alkylated species represent 24 percent of the tPAH concentration, and the ratio of alkylated to nonalkylated compounds is 19 . Both indicators are similar to those found in Watts Branch.

2. Pope Branch: In this stream, 56 percent of the tPAH concentration is made up of nonalkylated compounds, of which 86 percent are HMW compounds. Alkylated species represent 24 percent of the tPAH concentration; however, the ratio of alkylated to nonalkylated compounds $(0.70)$ is more like the ratio found in Hickey Run sediment. Pope Branch is characterized by a small percentage of nonalkylated species and a low ratio of alkylated/nonalkylated compounds (24).

3. Fort DuPont Creek: Most (77 percent) of the tPAH concentration is composed of nonalkylated compounds, of which 89 percent are HMW compounds. Alkylated species compose 21 percent of the total, and the ratio of alkylated to nonalkylated compounds is 27 .

4. Fort Stanton Creek: Most (77 percent) of the tPAH concentration is composed of nonalkylated compounds, 59 percent of which are HWM compounds. The alkylated species compose 32 percent of the total, with a ratio of alkylated to nonalkylated compounds of 27 , the same as in Fort DuPont Creek.

5. Other observations: Fluoranthene was the most abundant PAH in most of the samples collected in this study, although several samples had high concentrations of benzo(b)fluoranthene, chrysene, and pyrene. The HMW compounds clearly dominate the nonalkylated totals in all streams except Hickey Run, indicating pyrogenic sources for the PAHs (Steinhauer and Boehm, 1992). Hickey Run is dominated by LMW compounds, indicating a petrogenic source such as petroleum oils. When only storm samples are considered, the HWM compounds dominate but were present at percentages lower than those found in the other streams. Hickey Run also displayed the most variation in the compounds present at the highest concentrations, which include pyrene, C2- and C3-phenanthrenes/anthracenes, and benzo(b)fluoranthene. The ratio of the alkylated/nonalkylated compounds ranges from 19 in Nash Run to 56 in Hickey Run, with NWB and Watts Branch having similar high ratios (38), whereas the other tributaries have ratios of 24 to 28 . The concentrations of alkylated compounds were highest in Hickey Run (average total $13,000 \mu \mathrm{g} / \mathrm{kg}$ ), followed by NWB $(930 \mu \mathrm{g} / \mathrm{kg}$ ) (although the Hickey Run value may be skewed by the low-flow sample). Of the four suites of alkylated compounds, $\mathrm{C} 1-\mathrm{C} 4$ chrysene/benzo(a)anthracenes were present in the highest concentrations in NEB, LBDC, and Watts Branch, whereas concentrations of $\mathrm{C} 1-\mathrm{C} 4$ phenanthrenes/anthracenes were highest in NWB and Hickey Run. 


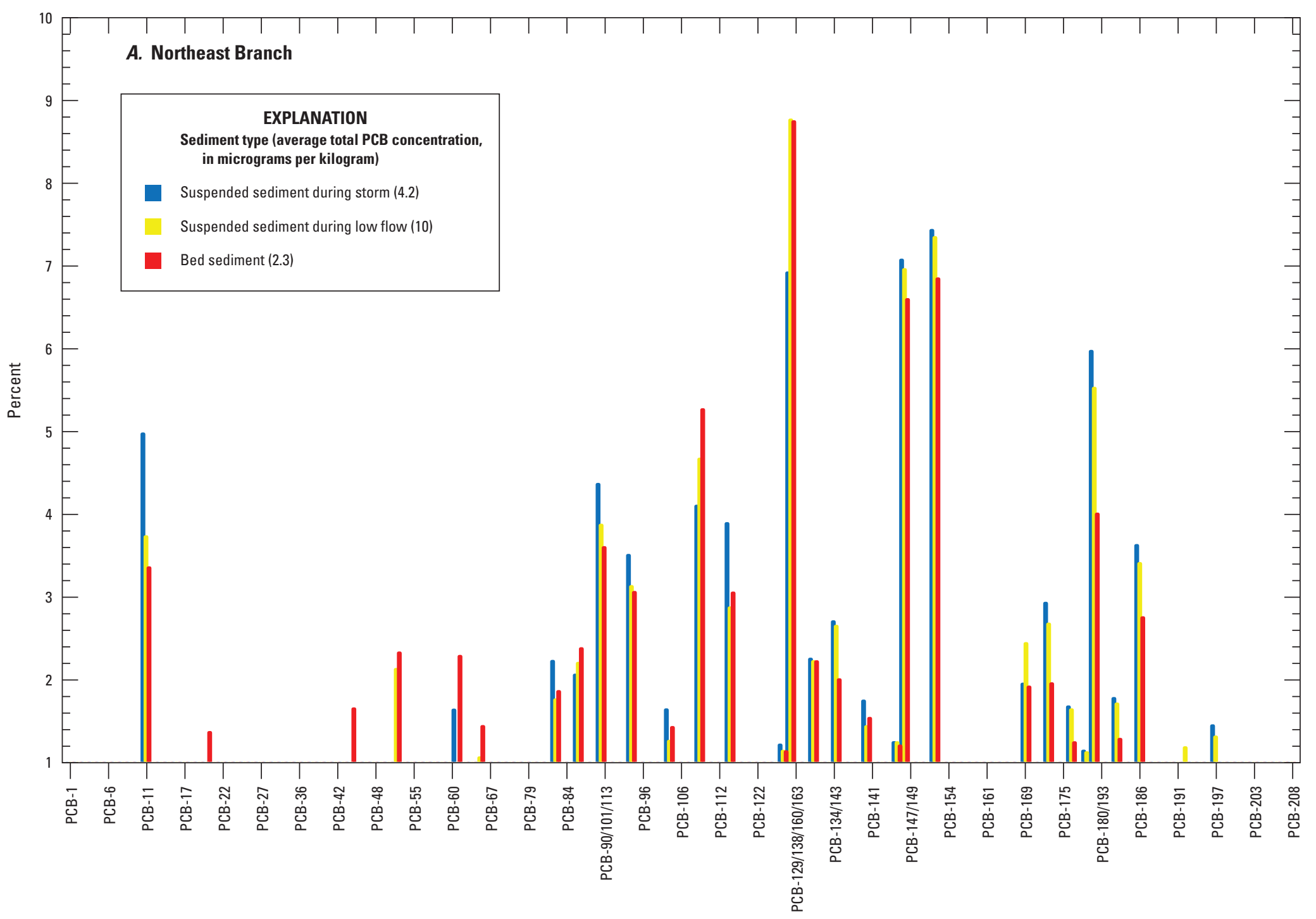

PCB congener

Figure 9. Molar percentage of polychlorinated biphenyls (PCBs) for PCB congeners in samples of suspended sediment during storm and low-flow conditions and samples of bed sediment from $A$, Northeast Branch, $B$, Northwest Branch, $C$, Beaverdam Creek, D, Watts Branch, E, Hickey Run, F, Nash Run, G, Pope Branch, $H$, Fort DuPont Creek, and $I$, Fort Stanton Creek. Only congeners present above 1 percent of total are shown. 


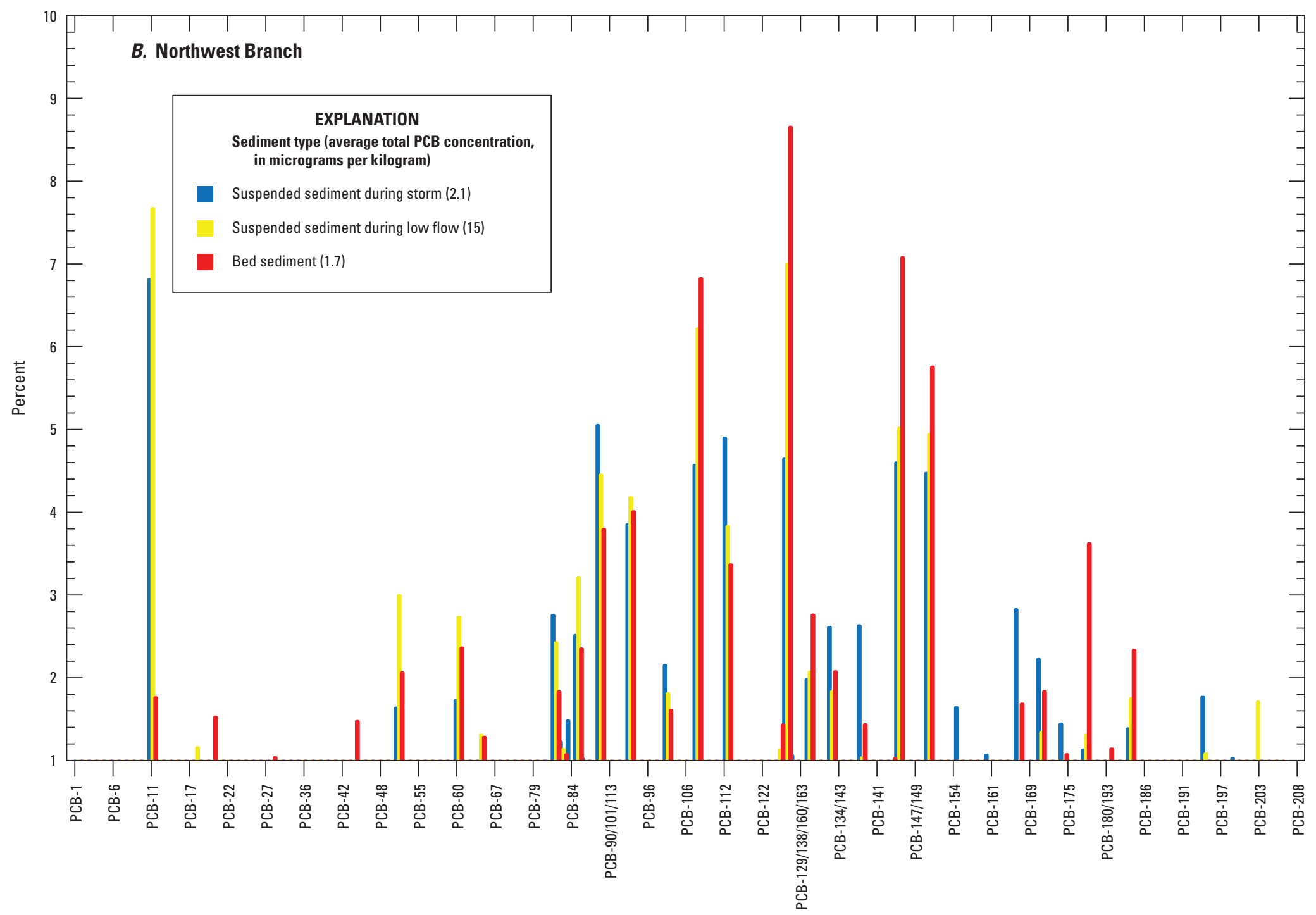

PCB congener

Figure 9. Molar percentage of polychlorinated biphenyls (PCBs) for PCB congeners in samples of suspended sediment during storm and low-flow conditions and samples of bed sediment from $A$, Northeast Branch, B, Northwest Branch, $C$, Beaverdam Creek, D, Watts Branch, E, Hickey Run, $F$, Nash Run, G, Pope 


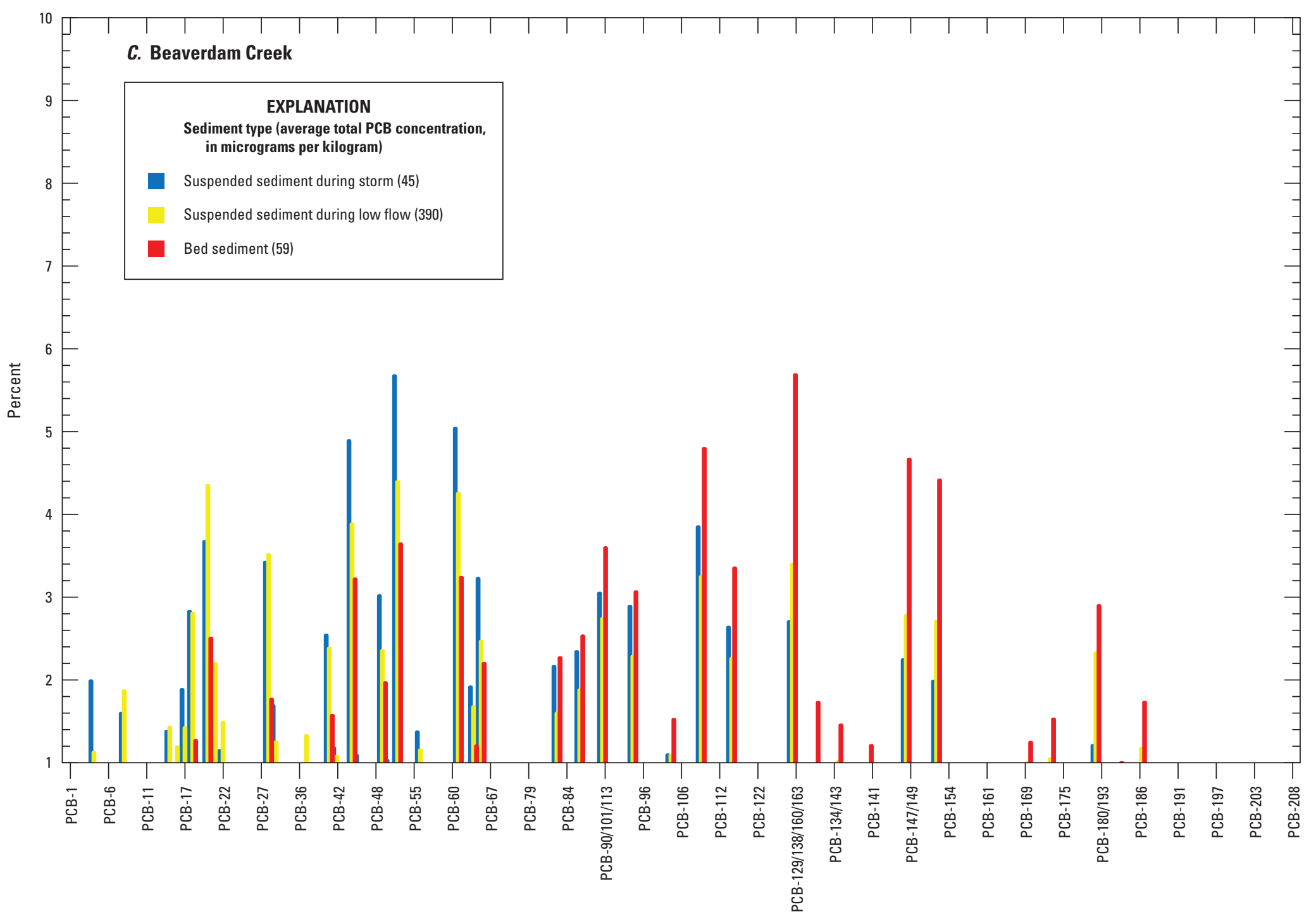

PCB congener

Figure 9. Molar percentage of polychlorinated biphenyls (PCBs) for PCB congeners in samples of suspended sediment during storm and low-flow conditions and samples of bed sediment from $A$, Northeast Branch, $B$, Northwest Branch, $C$, Beaverdam Creek, D, Watts Branch, E, Hickey Run, F, Nash Run, G, Pope Branch, H, Fort DuPont Creek, and I, Fort Stanton Creek. Only congeners present above 1 percent of total are shown.-Continued 


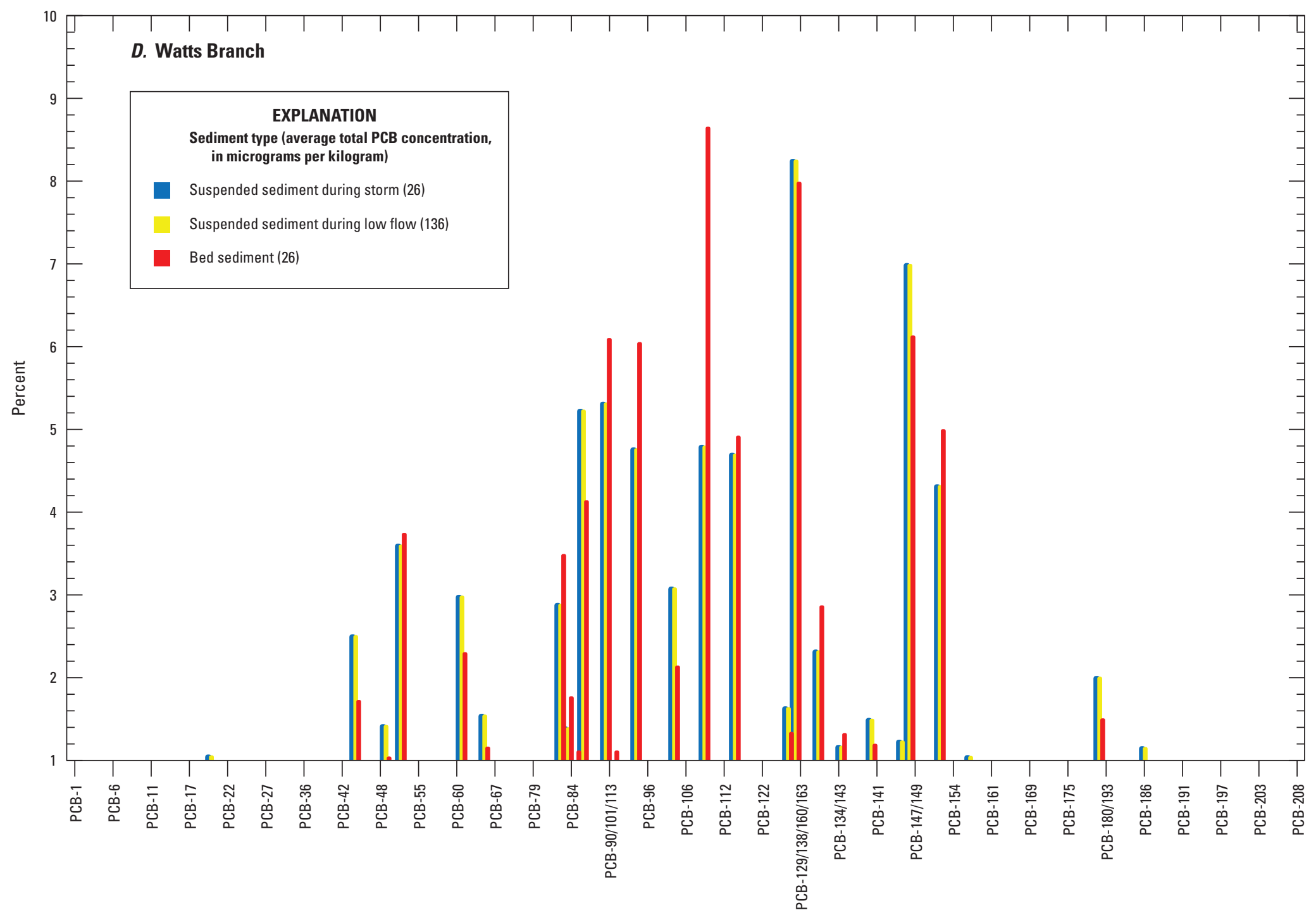

PCB congener

Figure 9. Molar percentage of polychlorinated biphenyls (PCBs) for PCB congeners in samples of suspended sediment during storm and low-flow conditions and samples of bed sediment from $A$, Northeast Branch, B, Northwest Branch, C, Beaverdam Creek, D, Watts Branch, E, Hickey Run, F, Nash Run, G, Pope 


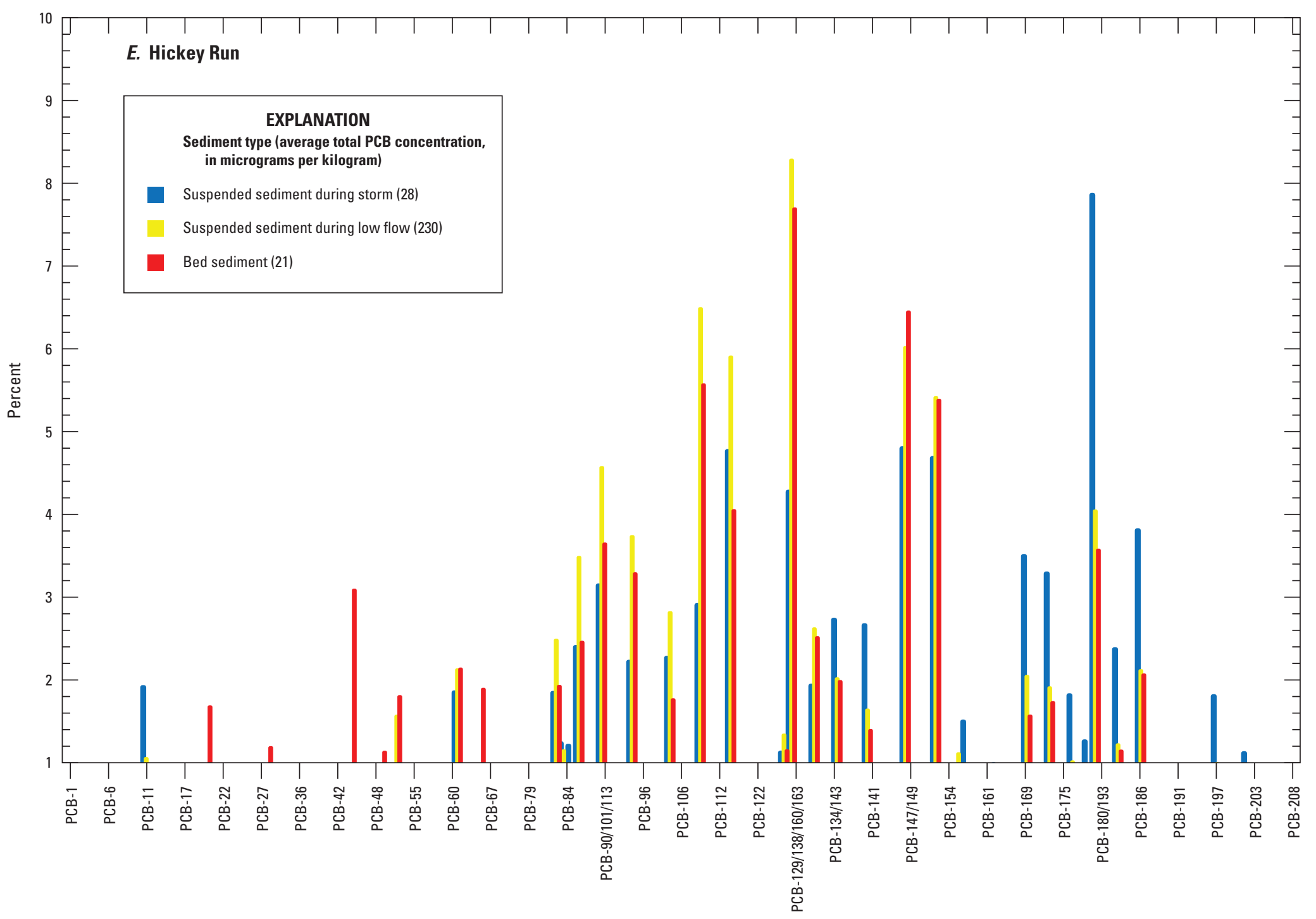

PCB congener

Figure 9. Molar percentage of polychlorinated biphenyls (PCBs) for PCB congeners in samples of suspended sediment during storm and low-flow conditions and samples of bed sediment from $A$, Northeast Branch, $B$, Northwest Branch, $C$, Beaverdam Creek, D, Watts Branch, E, Hickey Run, F, Nash Run, G, Pope Branch, H, Fort DuPont Creek, and I, Fort Stanton Creek. Only congeners present above 1 percent of total are shown.-Continued 


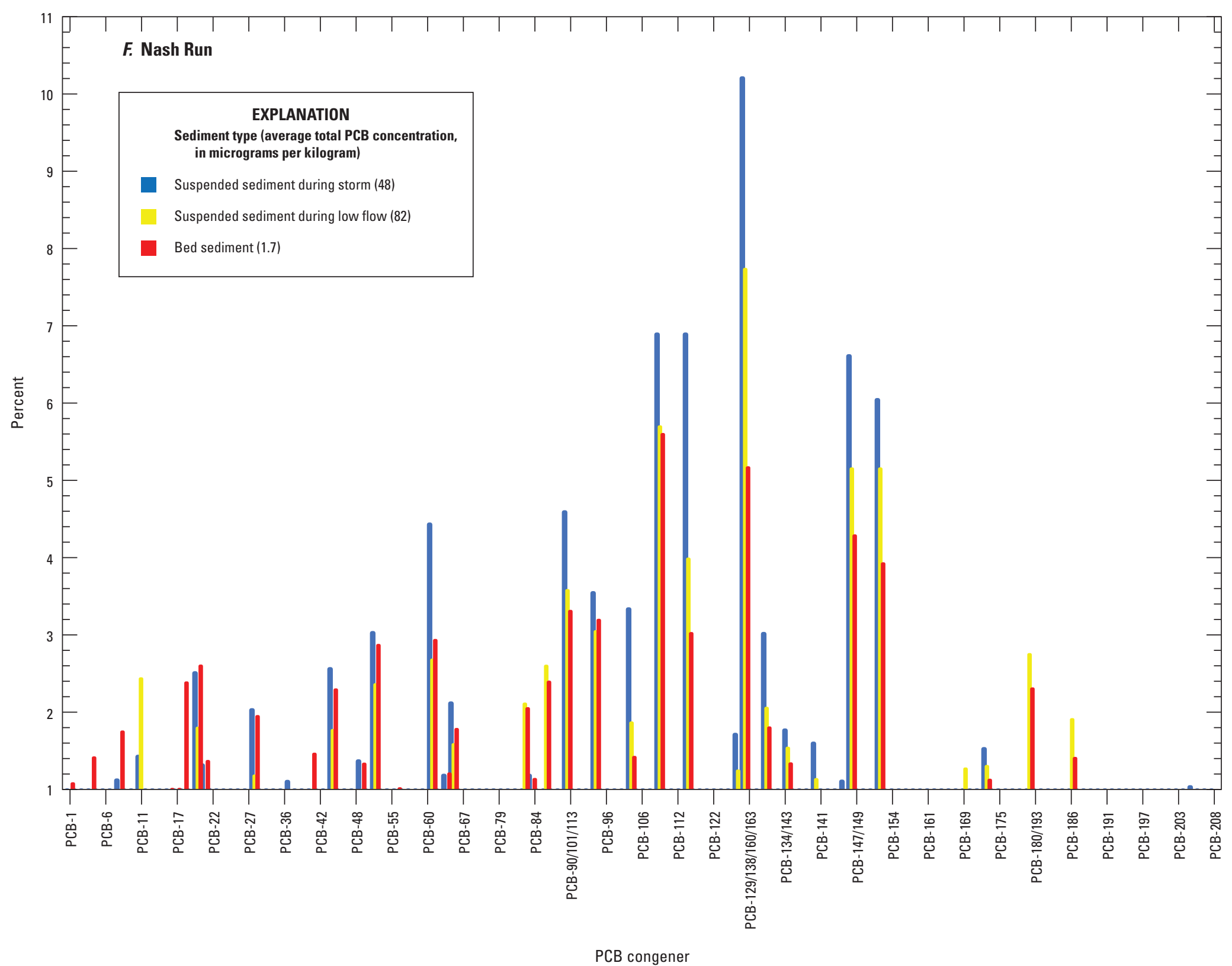

Figure 9. Molar percentage of polychlorinated biphenyls (PCBs) for PCB congeners in samples of suspended sediment during storm and low-flow conditions and samples of bed sediment from $A$, Northeast Branch, B, Northwest Branch, C, Beaverdam Creek, D, Watts Branch, E, Hickey Run, F, Nash Run, G, Pope Branch, H, Fort DuPont Creek, and I, Fort Stanton Creek. Only congeners present above 1 percent of total are shown.-Continued 


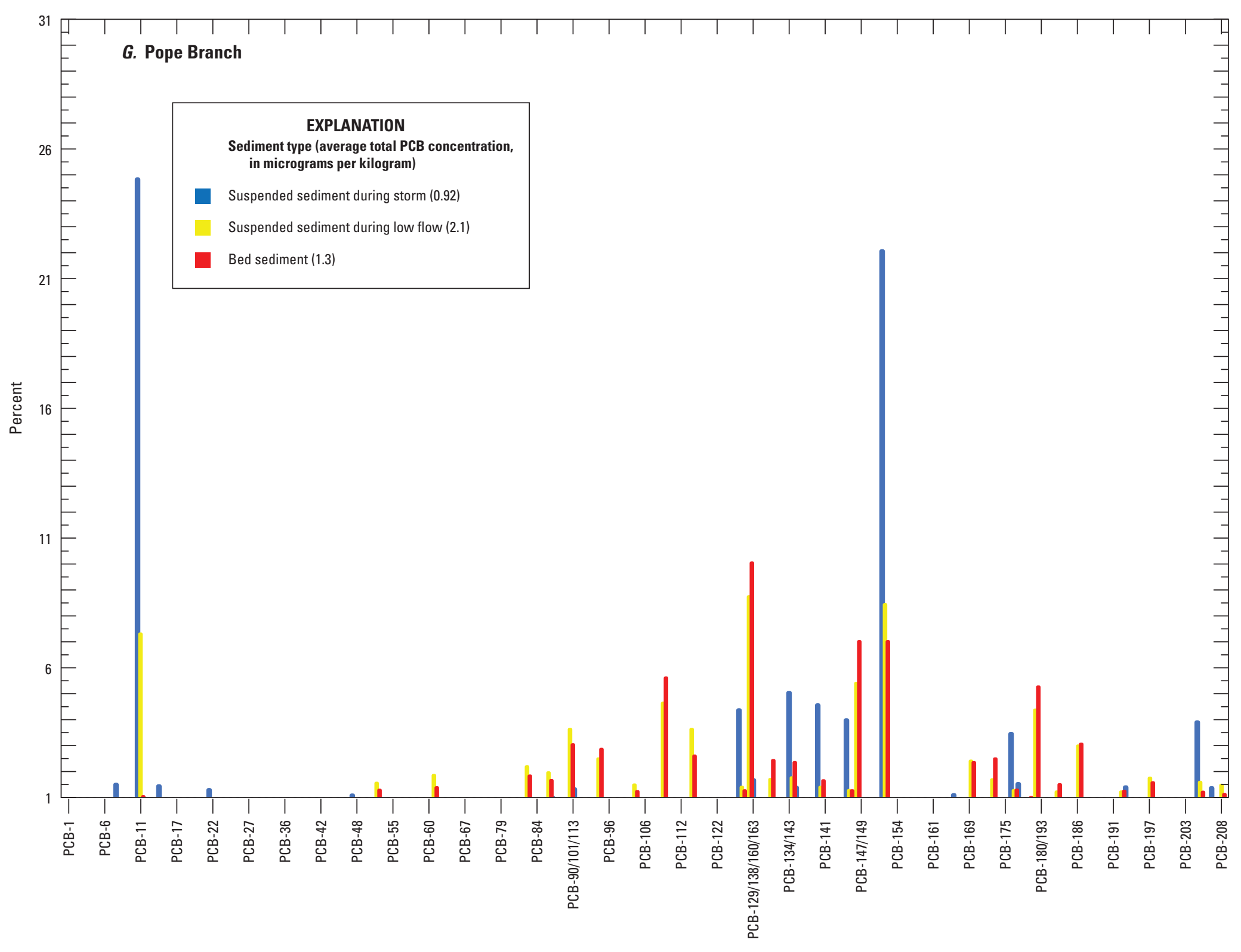

Figure 9. Molar percentage of polychlorinated biphenyls (PCBs) for PCB congeners in samples of suspended sediment during storm and low-flow conditions and samples of bed sediment from A, Northeast Branch, B, Northwest Branch, $C$, Beaverdam Creek, D, Watts Branch, E, Hickey Run, F, Nash Run, G, Pope Branch, H, Fort DuPont Creek, and I, Fort Stanton Creek. Only congeners present above 1 percent of total are shown.-Continued 


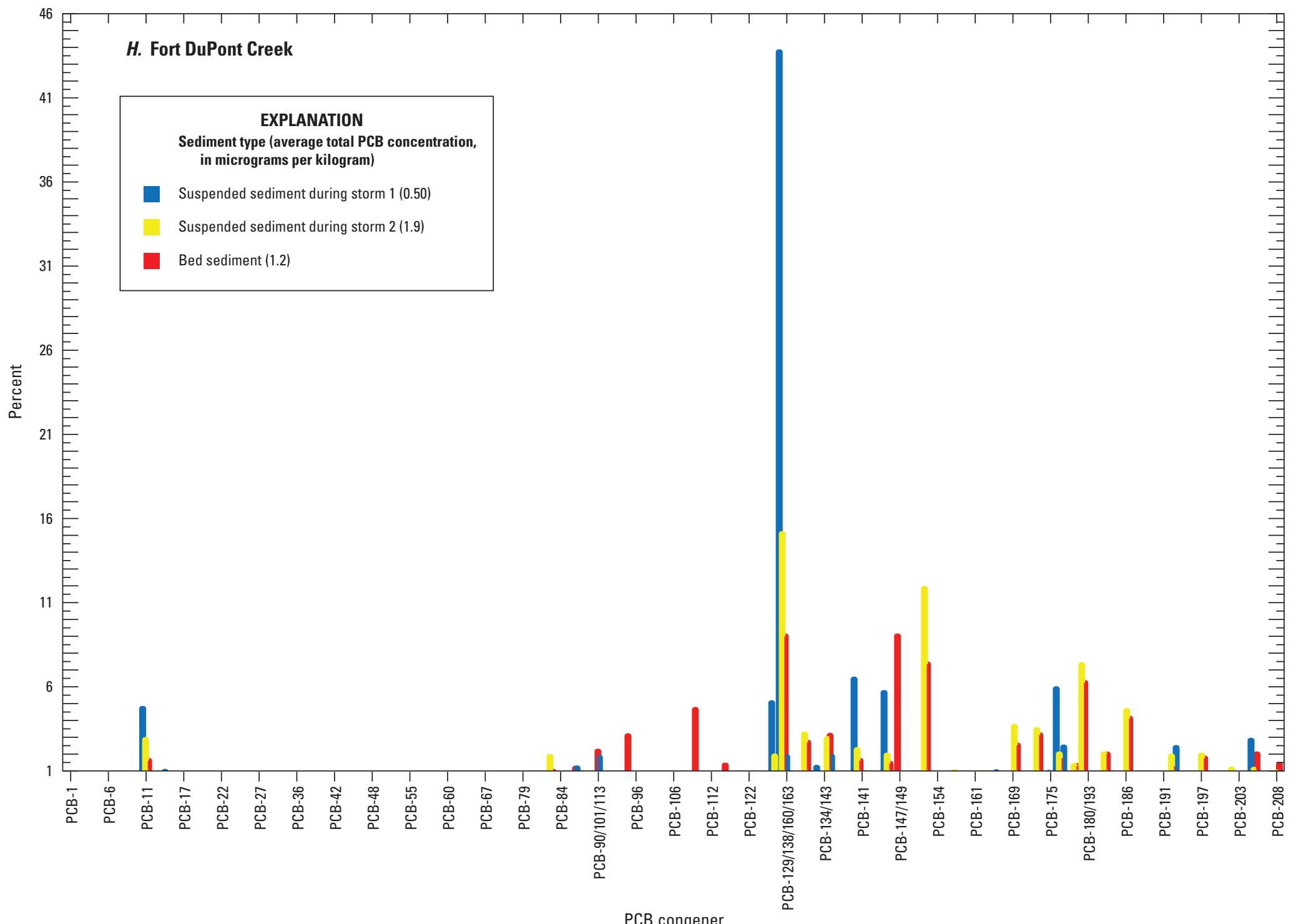

PCB congener

Figure 9. Molar percentage of polychlorinated biphenyls (PCBs) for PCB congeners in samples of suspended sediment during storm and low-flow conditions and samples of bed sediment from $A$, Northeast Branch, B, Northwest Branch, C, Beaverdam Creek, D, Watts Branch, E, Hickey Run, $F$, Nash Run, G, Pope Branch, H, Fort DuPont Creek, and I, Fort Stanton Creek. Only congeners present above 1 percent of total are shown.-Continued 


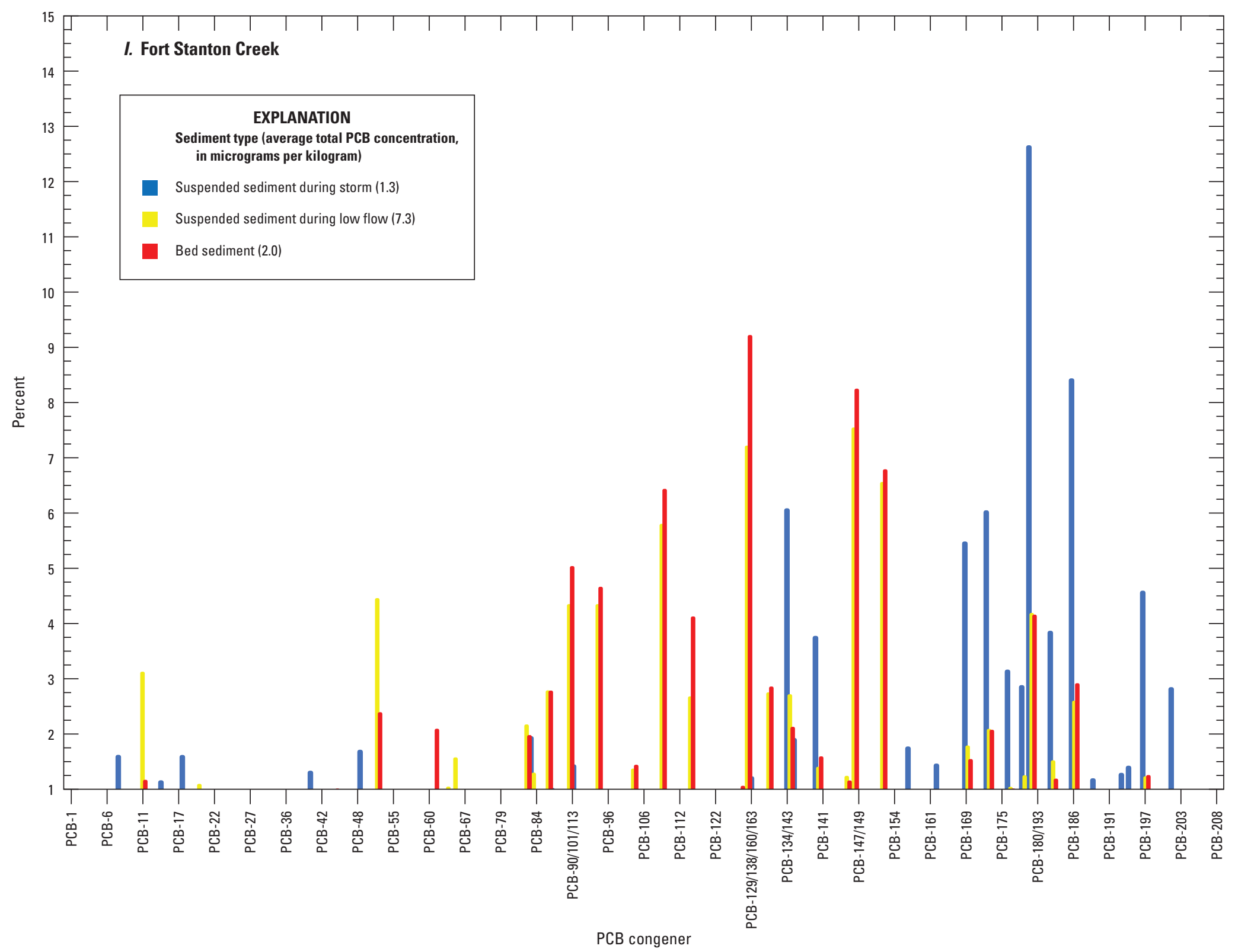

Figure 9. Molar percentage of polychlorinated biphenyls (PCBs) for PCB congeners in samples of suspended sediment during storm and low-flow conditions and samples of bed sediment from $A$, Northeast Branch, $B$, Northwest Branch, $C$, Beaverdam Creek, D, Watts Branch, E, Hickey Run, F, Nash Run, G, Pope Branch, H, Fort DuPont Creek, and I, Fort Stanton Creek. Only congeners present above 1 percent of total are shown.-Continued 


\section{Loads of Polychlorinated Biphenyls and Polycyclic Aromatic Hydrocarbons}

As described in the preceding section, the PCB makeup of the low-flow and storm sediment samples is similar. The calculated RMSE demonstrates that the combined differences in makeup of the low-flow and storm samples among the tributaries are less than 0.1 percent. Therefore, the average concentrations were considered suitable for use in calculating loadings.

Averaged concentrations of tPCB, tPAH, and TEQs in the low-flow and storm samples were multiplied by the sediment loadings to calculate the COC loadings for 2017 (table 25). The tPCB loading for 2017 was $820 \mathrm{~g}$; the respective loadings and their source tributaries (listed in order of magnitude) are LBDC, 590 g; NWB, 95 g; NEB, 60 g; Watts Branch, 25 g; and Hickey Run, 19 g. Proportionally, the PCB loading is distributed as follows: LBDC, 75 percent; NWB, 12 percent; NEB, 8 percent; Watts Branch, 3.2 percent; and Hickey Run, 2.4 percent. Toxicity loading of sediment, in TEQ, totaled $3.81 \times 10^{-3} \mathrm{~g} / \mathrm{yr}$, including LBDC, 47 percent; NEB, 37 precent; NWB, 7.6 percent; Hickey Run, 3.3 percent; and Watts Branch, 2.5 percent.

The total PAH loadings to the Anacostia River in 2017 (table 25) are estimated to be $89,000 \mathrm{~g}$, with $52,000 \mathrm{~g}$ from NWB; 20,000 $\mathrm{g}$ from NEB; 9,800 $\mathrm{g}$ from LBDC; 5,200 $\mathrm{g}$ from Hickey Run; and 1,400 g from Watts Branch. Nearly 60 percent of the total PAH loading to the Anacostia River originates from NWB, 23 percent from NEB, 11 percent from LBDC, 6 percent from Hickey Run, and 1.6 percent from Watts Branch. Regulatory concern is generally focused on the suite of nonalkylated compounds, and the loading of nonalkylated PAH totals $63,000 \mathrm{~g}$, which is 71 percent of the tPAH that includes the alkylated compounds. The nonalkylated PAH loadings range from 1,500 $\mathrm{g}$ in Hickey Run to $37,000 \mathrm{~g}$ in NWB.

\section{Loads of Polychlorinated Biphenyls and Polyaromatic Hydrocarbons in Ungaged Tributaries}

Sediment-bound PCB and PAH loads were also calculated for the small ungaged tributaries (table 25). As described previously, sediment loadings in these tributaries were established by using the loads calculated for Watts Branch, normalized to basin area. Because they have similar basin areas, Nash Run and Fort DuPont Creek have similar loadings $\left(1.2 \times 10^{5}\right.$ and $1.1 \times 10^{5} \mathrm{~kg} / \mathrm{yr}$, respectively), as do Pope Branch and Fort Stanton Creek $\left(6.2 \times 10^{4}\right.$ and $5.6 \times 10^{4} \mathrm{~kg} / \mathrm{yr}$, respectively). By using these sediment loadings and the average concentrations in the respective low-flow and storm samples, total loadings of PCBs and PAHs were calculated to be $8.1 \mathrm{~g} / \mathrm{yr}$ and $770 \mathrm{~g} / \mathrm{yr}$, respectively. Nash Run was the largest of the ungaged tributary sources, supplying 94 percent of the PCB and 85 percent of the PAH loadings (7.6 and $650 \mathrm{~g} / \mathrm{yr}$, respectively) of the ungaged tributaries. Although additional study would help to confirm the loading in Nash Run, the contribution from the ungaged streams is extremely small compared with the contribution from the gaged tributaries. For example, the PCB loading in Nash Run is less than one-third that in Watts Branch, which in turn makes up only about 3 percent of the total from all of the gaged streams.

\section{Comparison of Loads during Storms and Low Flow}

Loadings of sediment, PCBs, and PAHs were calculated for the individual storm and low-flow events (app. 1). Although the results for the individual storms were not used directly in calculating the yearly loadings, they demonstrate the relative magnitude of low-flow and stormflow contributions to the yearly loadings. During each storm event (app. 1, table 1.2), many thousands to millions of kilograms of sediment are transported, supplying grams of PCBs and thousands of grams of PAHs to the Anacostia River. During low flow, transported sediment masses are typically less than 100 kilograms per day $(\mathrm{kg} / \mathrm{d})$, and only a few milligrams of PCBs and a few grams of PAHs are ultimately transported per day to the Anacostia River.

For example, the loadings in NEB, NWB, LBDC, Watts Branch, and Hickey Run during the March 31, 2017, storm ( 0.95 in. of rain in 24 hours) were compared with the loads expected during low flow (table 26). Hydrographs and sedographs for this storm are presented in figure 8 . The stormflow loadings were calculated over a 48-hour period for all tributaries except LBDC; load calculations for LBDC ended at the beginning of the first tide cycle following peak flow.

The sediment loading during this storm totaled $5.81 \times 10^{5} \mathrm{~kg}$, with 33 percent from NEB, 39 percent from NWB, 25 percent from LBDC, 2 percent from Watts Branch, and 1 percent from Hickey Run. These contribution percentages are nearly identical to those calculated for the entire year of 2017. The tPCB loadings for the storm totaled $6.1 \mathrm{~g}$, most (82 percent) of which was derived from LBDC; the tPAH load was $920 \mathrm{~kg}$, with the largest percentage (46 percent) derived from NWB.

The storm loadings were normalized to daily mass delivered (in kilograms per day) to compare them with the lowflow loadings. Sediment loadings for a "storm-day" in each stream totaled $4.24 \times 10^{5} \mathrm{~kg} / \mathrm{d}$, and total PCB and PAH loadings were 7.9 and 670 grams per day (g/d), respectively.

To estimate the low-flow contribution, typical discharge and SSC values must be assigned to each stream. Low-flow discharges are obtained from USGS streamgaging data, and an SSC of $10 \mathrm{mg} / \mathrm{L}$ was assigned for each tributary. This concentration may be high for low flow but is comparable to the SSC predicted from the turbidity-SSC relations. For each tributary, the respective $\mathrm{COC}$ concentrations measured in the storm samples and the average of the concentrations measured in the two low-flow samples were used to calculate the chemical loads. 
Table 25. Loads and relative contribution of total polychlorinated biphenyls and polycyclic aromatic hydrocarbons in tributaries to the Anacostia River in 2017.

[kg, kilograms; g, grams; \%, percent; TEQ, toxic equivalency; PCB; polychlorinated biphenyls; PAH, polycyclic aromatic hydrocarbons; --, not applicable]

\begin{tabular}{|c|c|c|c|c|c|c|c|c|c|c|c|c|c|c|}
\hline \multirow[b]{2}{*}{ Tributary } & \multicolumn{7}{|c|}{ Load } & \multicolumn{7}{|c|}{ Relative contribution } \\
\hline & $\begin{array}{l}\text { Sediment } \\
(\mathbf{k g})\end{array}$ & $\begin{array}{c}\text { Total } \\
\text { PCB } \\
(g)\end{array}$ & $\begin{array}{l}\text { PCB } \\
\text { TEO } \\
(g)\end{array}$ & $\begin{array}{l}\text { Total } \\
\text { PAH } \\
(g)\end{array}$ & $\begin{array}{c}\text { Total } \\
\text { nonalkylated } \\
\text { PAH } \\
\text { (g) }\end{array}$ & $\begin{array}{l}\text { Total } \\
\text { alkylated } \\
\text { PAH } \\
\text { (g) }\end{array}$ & $\begin{array}{l}\text { PAH } \\
\text { TEQ } \\
(g)\end{array}$ & $\begin{array}{c}\text { Sediment } \\
\text { contribution } \\
(\%)\end{array}$ & $\begin{array}{l}\text { Total } \\
\text { PCB } \\
(\%)\end{array}$ & $\begin{array}{l}\text { PCB } \\
\text { TEQ } \\
(\%)\end{array}$ & $\begin{array}{l}\text { Total } \\
\text { PAH } \\
(\%)\end{array}$ & $\begin{array}{c}\text { Total } \\
\text { nonalkylated } \\
\text { PAH } \\
(\%)\end{array}$ & $\begin{array}{l}\text { Total } \\
\text { alkylated } \\
\text { PAH } \\
(\%)\end{array}$ & $\begin{array}{l}\text { PAH } \\
\text { TEQ } \\
(\%)\end{array}$ \\
\hline \multicolumn{15}{|c|}{ Gaged tributaries } \\
\hline Northeast Branch & $1.02 \times 10^{7}$ & 60 & $1.4 \times 10^{-3}$ & 20,000 & 16,000 & 4,100 & 1,600 & 32 & 7.6 & 37 & 23 & 25 & 16 & 26 \\
\hline Northwest Branch & $1.55 \times 10^{7}$ & 95 & $3.1 \times 10^{-4}$ & 52,000 & 37,000 & 14,000 & 3,700 & 51 & 12 & 8.2 & 59 & 59 & 58 & 59 \\
\hline Beaverdam Creek & $4.45 \times 10^{6}$ & 590 & $1.8 \times 10^{-3}$ & 9,800 & 7,300 & 2,200 & 740 & 14 & 75 & 47 & 11 & 12 & 9.2 & 12 \\
\hline Watts Branch & $5.62 \times 10^{5}$ & 25 & $9.7 \times 10^{-5}$ & 1,400 & 1,000 & 370 & 100 & 2 & 3.2 & 2.5 & 1.6 & 1.6 & 1.5 & 1.7 \\
\hline Hickey Run & $2.82 \times 10^{5}$ & 19 & $1.3 \times 10^{-4}$ & 5,200 & 1,500 & 3,600 & 130 & 1 & 2.4 & 3.3 & 5.9 & 2.4 & 15 & 2.0 \\
\hline Total & $3.10 \times 10^{7}$ & 790 & $3.810 \times 10^{-3}$ & 88,000 & 63,000 & 24,000 & 6,300 & 100 & 100 & 100 & 100 & 100 & 100 & 100 \\
\hline \multicolumn{15}{|c|}{ Ungaged streams } \\
\hline Nash Run & $1.2 \times 10^{5}$ & 7.6 & $2.1 \times 10^{-5}$ & 650 & 490 & 160 & 52 & 34 & 94 & 78 & 85 & 86 & 82 & 88 \\
\hline Pope Branch & $6.2 \times 10^{4}$ & 0.093 & $1.3 \times 10^{-7}$ & 39 & 23 & 16 & 2.1 & 18 & 1.2 & 0.50 & 5.1 & 4.0 & 8.1 & 3.6 \\
\hline Fort DuPont Creek & $1.1 \times 10^{5}$ & 0.13 & $4.2 \times 10^{-5}$ & 39 & 31 & 8.4 & 3.0 & 32 & 1.7 & 16 & 5.1 & 5.4 & 4.3 & 5.1 \\
\hline Fort Stanton Creek & $5.6 \times 10^{4}$ & 0.24 & $1.5 \times 10^{-6}$ & 34 & 26 & 11 & 2.2 & 16 & 3.0 & 5.5 & 4.7 & 4.6 & 5.8 & 3.7 \\
\hline Total & $3.5 \times 10^{5}$ & 8.1 & $2.7 \times 10^{-5}$ & 770 & 570 & 200 & 59 & 100 & 100 & 100 & 100 & 100 & 100 & 100 \\
\hline Total for all tributaries & $3.2 \times 10^{7}$ & 820 & $3.8 \times 10^{-3}$ & 89,000 & 64,000 & 25,000 & 6,300 & -- & -- & -- & -- & -- & -- & -- \\
\hline
\end{tabular}

${ }^{1}$ Loads of chemicals were calculated by using the average concentration in all samples collected in this study. 
Table 26. Summary of sediment and chemical concentrations and loads in tributaries for storm event on March 31, 2017, compared with low-flow loads.

[L, liters; L/d, liters per day; kg/d, kilograms per day; $\mu \mathrm{g} / \mathrm{kg}$, micrograms per kilograms; g, grams; g/d, grams per day; $\mathrm{kg}$, kilograms; $\mathrm{ft}^{3} / \mathrm{s}$, cubic feet per second; mg/L, milligrams per liter; PCB, polychlorinated biphenyls; PAH, polycyclic aromatic hydrocarbons; SSC, suspended-sediment concentration; ,-- not applicable]

\begin{tabular}{|c|c|c|c|c|c|c|}
\hline Storm characteristic & $\begin{array}{c}\text { Northeast } \\
\text { Branch }\end{array}$ & $\begin{array}{c}\text { Northwest } \\
\text { Branch }\end{array}$ & $\begin{array}{c}\text { Beaverdam } \\
\text { Creek }\end{array}$ & $\begin{array}{l}\text { Watts } \\
\text { Branch }\end{array}$ & $\begin{array}{c}\text { Hickey } \\
\text { Run }\end{array}$ & Total \\
\hline \multicolumn{7}{|c|}{ Stormflow } \\
\hline Date/time event ended & 4/2 0:00 & 4/2 0:00 & $3 / 3119: 20$ & 4/2 0:00 & 4/2 0:00 & -- \\
\hline Duration (minutes) & 2,885 & 2,885 & 965 & 2,885 & 2,885 & -- \\
\hline Total sediment load (kg/d) & $1.02 \times 10^{5}$ & $1.11 \times 10^{5}$ & $2.02 \times 10^{5}$ & 5,300 & 3,700 & $4.24 \times 10^{5}$ \\
\hline PCB concentration $(\mu \mathrm{g} / \mathrm{kg})$ & 1.4 & 3.1 & 36 & 5.1 & 20 & -- \\
\hline Total PAH concentration $(\mu \mathrm{g} / \mathrm{kg})$ & 1,100 & 1,900 & 1,600 & 1,200 & 6,000 & -- \\
\hline Total PCB load (g) & 0.29 & 0.69 & 4.9 & 0.055 & 0.15 & 6.1 \\
\hline \multicolumn{7}{|c|}{ Low flow } \\
\hline Low-flow discharge $\left(\mathrm{ft}^{3} / \mathrm{s}\right)$ & 20 & 15 & 8 & 1.5 & 1 & -- \\
\hline Discharge (L/day) & $4.89 \times 10^{7}$ & $3.67 \times 10^{7}$ & $1.96 \times 10^{7}$ & $3.67 \times 10^{6}$ & $2.45 \times 10^{6}$ & $1.11 \times 10^{8}$ \\
\hline Typical SSC (mg/L) & 10 & 10 & 10 & 10 & 10 & -- \\
\hline Total sediment load $(\mathrm{kg} / \mathrm{d})$ & 489 & 367 & 196 & 37 & 25 & 1,100 \\
\hline PCB concentration in LF $(\mu \mathrm{g} / \mathrm{kg})$ & 9.8 & 14 & 380 & 78 & 230 & -- \\
\hline PAH concentration in LF $(\mu \mathrm{g} / \mathrm{kg})$ & 1,500 & 5,800 & 3,800 & 2,900 & 84,000 & -- \\
\hline Total PCB load $(\mathrm{g} / \mathrm{d})$ & 0.0048 & 0.0051 & 0.075 & 0.0029 & 0.0056 & 0.093 \\
\hline Total PAH load (g/d) & 0.73 & 2.1 & 0.74 & 0.11 & 2.1 & 5.8 \\
\hline
\end{tabular}

The sediment mass transported during low flow totaled $1,100 \mathrm{~kg} / \mathrm{d}$, which is 0.20 percent of the sediment load calculated for the March storm $\left(5.81 \times 10^{5} \mathrm{~kg}\right)$. These values indicate that more than 500 days of low flow would be required to deliver the same sediment mass that was delivered during the March storm. This value (500 days) is the number of "equivalent base-flow days" for the storm (Wilson and Bonin, $2007,2008)$. The tPCB load during low flow is estimated to be $0.093 \mathrm{~g} / \mathrm{d}$ (93 milligrams per day), so 85 days of low flow would be needed to deliver the total PCB load delivered in the storm $(7.9 \mathrm{~g} / \mathrm{d})$. The tPAH load during low flow is estimated to be $5.8 \mathrm{~g} / \mathrm{d}$, which is 0.9 percent of the total delivered in the storm $(670 \mathrm{~g} / \mathrm{d})$, a load that would be delivered in 113 equivalent base-flow days. For example, in LBDC, the storm PCB concentration $(36 \mu \mathrm{g} / \mathrm{kg})$ is approximately 10 percent of the low-flow concentration $(380 \mu \mathrm{g} / \mathrm{kg})$. Low-flow concentrations are uniformly higher than storm concentrations because of the incorporation of "cleaner" sediment, especially sand, in the storm load. Low-flow sediment is typically the finest grained materials (clays and particulate organic matter) which, because of their associated carbon content and high surface area, preferentially sequester hydrophobic compounds. Additionally, particulates associated with engine exhaust are extremely fine grained, and typically contain various PAH compounds.

\section{Pesticide Concentrations and Loads}

Pesticides are considered separately from the other COCs because of the difficulties encountered with the analyses. The pesticide data show a wide range of MDLs among the lowflow, storm, and bed-sediment analyses. The highest MDLs were reported for the suspended sediment and, as a result, many compounds were reported as nondetected. Only chlordane was found in the suspended sediment at concentrations 
above the MDL (app. 2, table 2.3). The bed-sediment data were reported at much lower MDLs and, as a result, many more compounds were quantified. Table 27 lists the MDLs for the different types of samples collected from LBDC during low flow and the March 2017 storm event and the associated bed-sediment sample. MDLs for the low-flow samples are up to 4,000 times greater than those reported for the bed sediment. MDLs for the suspended sediment collected during storms are approximately 30 times those reported for bed sediment. The high MDLs for suspended sediment result from matrix interferences and moisture in the sediment during extraction, and other factors such as the larger mass of bed sediment available for analysis. Many pesticide compounds were quantifiable in bed sediment; however, it is reasonable to assume that pesticides measured in the bed sediment were also present at similar concentrations in the suspended sediment.

Table 27. Method detection levels for pesticides in suspendedsediment and bed-sediment samples collected from Beaverdam Creek.

[MDL, method detection level; $\mu \mathrm{g} / \mathrm{kg}$, micrograms per kilogram; na, not analyzed]

\begin{tabular}{|c|c|c|c|}
\hline \multirow{2}{*}{ Pesticide } & $\begin{array}{l}\text { TS-LBC- } \\
\text { SS-W- } \\
052417\end{array}$ & $\begin{array}{c}\text { TS-LBC- } \\
\text { LVSSD-D- } \\
101817\end{array}$ & $\begin{array}{l}\text { TS-LBC- } \\
\text { LVSSD-W- } \\
050517\end{array}$ \\
\hline & $\begin{array}{c}\text { Bed sediment } \\
\text { MDL } \\
(\mu \mathrm{g} / \mathrm{kg})\end{array}$ & $\begin{array}{c}\text { Low-flow } \\
\text { MDL } \\
(\mu \mathrm{g} / \mathrm{kg})\end{array}$ & $\begin{array}{c}\text { Storm } \\
\text { MDL } \\
\text { (pg/kg) }\end{array}$ \\
\hline 4,4'-DDD & 0.054 & 230 & 7.3 \\
\hline 4,4'-DDE & 0.17 & 85 & 2.7 \\
\hline 4,4'-DDT & 0.054 & 99 & 3.1 \\
\hline Aldrin & 0.056 & 170 & 5.3 \\
\hline alpha-BHC & 0.16 & 110 & 3.6 \\
\hline beta-BHC & 0.12 & 280 & 8.7 \\
\hline Chlordane & 0.60 & 850 & 17 \\
\hline delta-BHC & 0.19 & 92 & 2.9 \\
\hline Dieldrin & 0.05 & 64 & 2.0 \\
\hline Endosulfan I & 0.034 & 92 & 2.9 \\
\hline Endosulfan II & 0.16 & 130 & 4.0 \\
\hline Endosulfan sulfate & 0.068 & 85 & 2.7 \\
\hline Endrin & 0.15 & 99 & 3.1 \\
\hline Endrin aldehyde & 0.16 & 130 & 4.0 \\
\hline Endrine ketone & na & 78 & 2.4 \\
\hline gamma-BHC (Lindane) & 0.11 & 200 & 6.4 \\
\hline Heptachlor & 0.046 & 55 & 1.7 \\
\hline Heptachlor epoxide & 0.063 & 170 & 5.3 \\
\hline Methoxychlor & na & 85 & 2.7 \\
\hline Toxaphene & 17 & 2,600 & 82 \\
\hline
\end{tabular}

The maximum concentration in the aggregated set of suspended-sediment and bed-sediment samples was used to develop a maximum loading for each stream. The aggregated concentrations used for load calculations, and the sample types associated with the highest concentration, are presented in appendix 2, tables 2.2 to 2.4 .

Chlordane is of special interest in the Anacostia River because previous studies had identified high chlordane concentrations throughout the various tributaries (Phelps, 1993, 2005; Velinsky and others, 2011). All the chlordane concentrations used in the loading calculations were measured in suspended sediment. Maximum chlordane concentrations used for loadings, ranked in order from highest to lowest, are as follows: Watts Branch, $100 \mu \mathrm{g} / \mathrm{kg}$; LBDC, $70 \mu \mathrm{g} / \mathrm{kg}$; NEB, $42 \mu \mathrm{g} / \mathrm{kg}$; Hickey Run, $40 \mu \mathrm{g} / \mathrm{kg}$; and NWB, $21 \mu \mathrm{g} / \mathrm{kg}$ (app. 2 , table 2.3). Chlordane was not quantifiable in any of the ungaged streams. The suspended sediment was found to have quantifiable concentrations of dieldrin, endrin aldehyde, 4,4DDT, gamma-BHC, and methoxychlor, although not consistently in all streams. The suite of DDT compounds $\left(4,4^{\prime}\right.$-DDT, 4,4'-DDE, and 4,4'-DDD) was present in all bed-sediment samples, including bed sediment from the small ungaged tributaries. Notable high concentrations of pesticides are aldrin in NEB $(6.1 \mu \mathrm{g} / \mathrm{kg})$, dieldrin in NWB $(56 \mu \mathrm{g} / \mathrm{kg})$, methoxychlor in LBDC ( $46 \mu \mathrm{g} / \mathrm{kg}), 4,4-D D T$ in Hickey Run $(23 \mu \mathrm{g} / \mathrm{kg})$, and 4-4'-DDT $(140 \mu \mathrm{g} / \mathrm{kg})$ and methoxychlor $(103 \mu \mathrm{g} / \mathrm{kg})$ in Watts Branch. In the ungaged tributaries, 4-4'-DDD $(5.4 \mu \mathrm{g} / \mathrm{kg})$, 4,4'-DDE $(17 \mu \mathrm{g} / \mathrm{kg})$, and aldrin $(21 \mu \mathrm{g} / \mathrm{kg})$ were found in Pope Branch.

Table 28 presents the maximum pesticide loadings for the individual streams, and table 29 lists the total maximum tributary loads of pesticides. Because maximum concentrations were used to calculate these loads, these loadings should be considered maximums, especially given the limited dataset available. The highest yearly loadings for pesticides are 1,100 $\mathrm{g} / \mathrm{yr}$ for chlordane, $870 \mathrm{~g} / \mathrm{yr}$ for dieldrin, $200 \mathrm{~g} / \mathrm{yr}$ for endrin aldehyde, and $140 \mathrm{~g} / \mathrm{yr}$ for 4,4'-DDT (table 29). The three highest loadings for each tributary (table 28) are as follows:

- NEB: chlordane (430 g/yr), methoxychlor (16 g/yr), and 4,4'-DDD (8.1 g/yr)

- NWB: dieldrin (860 g/yr), chlordane (320 g/yr), and endrin aldehyde (150 g/yr)

- LBDC: chlordane (310 g/yr), methoxychlor (205 g/yr), and heptachlor epoxide ( $33 \mathrm{~g} / \mathrm{yr})$

- Watts Branch: 4,4'-DDT (79 g/yr), methoxychlor $(58 \mathrm{~g} / \mathrm{yr})$, and chlordane $(56 \mathrm{~g} / \mathrm{yr})$

- Hickey Run: chlordane (11 g/yr), 4,4'-DDT (6.5 g/yr), and 4,4'-DDE (1.6 g/yr).

Of the ungaged streams, only Pope Branch was found to contribute any pesticide at a yearly load of more than $1 \mathrm{~g} / \mathrm{yr}$ (4,4'-DDT, $1.3 \mathrm{~g} / \mathrm{yr}$; and 4,4'-DDE, $1.1 \mathrm{~g} / \mathrm{yr}$ ). 
Table 28. Maximum loads of pesticides in tributaries to the Anacostia River, 2017.

[g/yr, grams per year; g/d, grams per day; kg/yr, kilograms per year; ND, compound not detected; <, less than]

\begin{tabular}{|c|c|c|c|c|c|c|c|c|c|c|}
\hline \multirow{2}{*}{$\begin{array}{c}\text { Sediment, pesticide, and } \\
\text { total organic carbon }\end{array}$} & \multicolumn{2}{|c|}{ Northeast Branch } & \multicolumn{2}{|c|}{ Northwest Branch } & \multicolumn{2}{|c|}{ Beaverdam Creek } & \multicolumn{2}{|c|}{ Watts Branch } & \multicolumn{2}{|c|}{ Hickey Run } \\
\hline & $(g / y r)$ & $(g / d)$ & $(g / y r)$ & (g/yr) & $(g / y r)$ & $(g / d)$ & $(g / y r)$ & $(g / y r)$ & $(g / y r)$ & $(g / d)$ \\
\hline Sediment $^{1}$ & $1.02 \times 10^{7}$ & 27,900 & $1.55 \times 10^{7}$ & 42,500 & $4.45 \times 10^{6}$ & 12,200 & $5.62 \times 10^{5}$ & 1,540 & $2.82 \times 10^{5}$ & 773 \\
\hline 4,4'-DDD & 8.1 & 0.022 & 7.1 & 0.019 & 9.8 & 0.027 & 1.0 & 0.003 & 1.3 & 0.004 \\
\hline 4,4'-DDE & 2.6 & 0.007 & 17 & 0.047 & 6.7 & 0.018 & 1.5 & 0.004 & 1.6 & 0.004 \\
\hline 4,4'-DDT & ND & ND & 49 & 0.13 & 8.9 & 0.024 & 79 & 0.22 & 6.5 & 0.018 \\
\hline Aldrin & 62 & 0.17 & ND & ND & ND & $\mathrm{ND}$ & 0.00 & 0.000 & ND & ND \\
\hline alpha-BHC & ND & ND & ND & ND & ND & ND & 0.00 & 0.000 & ND & ND \\
\hline beta-BHC & ND & ND & ND & ND & ND & ND & 0.00 & 0.000 & ND & ND \\
\hline Chlordane & 430 & 1.2 & 320 & 0.88 & 310 & 0.85 & 56 & 0.15 & 11 & 0.048 \\
\hline delta-BHC & ND & ND & ND & ND & ND & ND & 14. & 0.040 & ND & ND \\
\hline Dieldrin & 4.7 & 0.013 & 860 & 2.4 & 1.7 & 0.005 & 0.34 & 0.001 & 0.25 & 0.001 \\
\hline Endosulfan I & $\mathrm{ND}$ & ND & ND & ND & ND & ND & 0.00 & 0.000 & $\mathrm{ND}$ & ND \\
\hline Endosulfan II & ND & ND & ND & ND & ND & ND & 0.00 & 0.000 & ND & ND \\
\hline Endosulfan sulfate & ND & ND & ND & ND & ND & ND & 0.00 & 0.000 & ND & ND \\
\hline Endrin & ND & ND & $\mathrm{ND}$ & ND & ND & ND & 0.41 & 0.001 & 0.18 & $<0.001$ \\
\hline Endrin aldehyde & ND & ND & 150 & 0.41 & ND & ND & 55 & 0.15 & 0.042 & $<0.001$ \\
\hline Endrin ketone & ND & ND & ND & ND & ND & ND & 0.00 & 0.000 & ND & ND \\
\hline gamma-BHC (Lindane) & ND & ND & ND & ND & 1.7 & 0.005 & 0.00 & 0.000 & ND & ND \\
\hline Heptachlor & 1.7 & 0.005 & 2.8 & 0.008 & 0.98 & 0.003 & 0.00 & 0.000 & 0.037 & $<0.001$ \\
\hline Heptachlor epoxide & 3.2 & 0.009 & 3.2 & 0.009 & 33 & 0.092 & 0.17 & 0.001 & 0.15 & $<0.001$ \\
\hline Total organic carbon ${ }^{1}$ & 75,000 & 210 & 170,000 & 470 & 40,000 & 110 & 2,100 & 5.8 & 3,700 & 10 \\
\hline Toxaphene & ND & ND & ND & ND & ND & ND & 0.00 & 0.000 & ND & ND \\
\hline Methoxychlor & 16 & 0.044 & ND & ND & 205 & 0.56 & 58 & 0.160 & ND & ND \\
\hline
\end{tabular}


Table 28. Maximum loads of pesticides in tributaries to the Anacostia River, 2017.-Continued

[g/yr, grams per year; g/d, grams per day; kg/yr, kilograms per year; ND, compound not detected; <, less than]

\begin{tabular}{|c|c|c|c|c|c|c|c|c|}
\hline \multirow{2}{*}{$\begin{array}{l}\text { Sediment, pesticide, and } \\
\text { total organic carbon }\end{array}$} & \multicolumn{2}{|c|}{ Nash Run } & \multicolumn{2}{|c|}{ Pope Branch } & \multicolumn{2}{|c|}{ Fort DuPont Creek } & \multicolumn{2}{|c|}{ Fort Stanton Creek } \\
\hline & $(g / y r)$ & $(g / d)$ & (g/yr) & $(g / y r)$ & (g/yr) & $(g / d)$ & (g/yr) & $(g / y r)$ \\
\hline Sediment $^{1}$ & $1.18 \times 10^{5}$ & 323 & $6.18 \times 10^{4}$ & 169 & $1.12 \times 10^{5}$ & 307 & $5.62 \times 10^{4}$ & 154 \\
\hline 4,4'-DDD & 0.027 & $<0.001$ & 0.33 & 0.001 & 0.12 & $<0.001$ & 0.023 & $<0.001$ \\
\hline 4,4'-DDE & ND & ND & 1.05 & 0.003 & 0.50 & 0.001 & 0.020 & $<0.001$ \\
\hline 4,4'-DDT & ND & ND & 1.3 & 0.004 & 0.12 & 0.000 & 0.035 & 0.000 \\
\hline Aldrin & ND & ND & ND & ND & ND & ND & ND & ND \\
\hline alpha-BHC & ND & ND & ND & ND & ND & ND & ND & ND \\
\hline beta-BHC & ND & ND & ND & ND & ND & ND & ND & ND \\
\hline Chlordane & ND & ND & ND & ND & ND & ND & ND & ND \\
\hline delta-BHC & ND & ND & ND & ND & ND & ND & ND & ND \\
\hline Dieldrin & 0.005 & $<0.001$ & ND & ND & ND & ND & ND & ND \\
\hline Endosulfan I & ND & ND & ND & ND & ND & ND & ND & ND \\
\hline Endosulfan II & ND & ND & ND & ND & ND & ND & ND & ND \\
\hline Endosulfan sulfate & ND & ND & ND & $\mathrm{ND}$ & ND & ND & ND & ND \\
\hline Endrin & ND & ND & 0.006 & $<0.001$ & ND & ND & ND & ND \\
\hline Endrin aldehyde & ND & ND & ND & ND & ND & ND & ND & ND \\
\hline Endrin ketone & ND & ND & ND & ND & ND & ND & ND & ND \\
\hline gamma-BHC (Lindane) & ND & ND & ND & ND & ND & ND & ND & ND \\
\hline Heptachlor & ND & ND & ND & ND & ND & ND & ND & ND \\
\hline Heptachlor epoxide & ND & ND & 0.017 & $<0.001$ & ND & ND & ND & ND \\
\hline Total organic carbon ${ }^{1}$ & 672 & 1.8 & 142 & 0.39 & 314 & 0.86 & 258 & 0.71 \\
\hline Toxaphene & ND & ND & ND & ND & ND & ND & ND & ND \\
\hline Methoxychlor & ND & ND & ND & ND & ND & ND & ND & ND \\
\hline
\end{tabular}

${ }^{1}$ Sediment and total organic carbon loads are in $\mathrm{kg} / \mathrm{yr}$. 


\section{Metal Concentrations and Loads}

Metal concentrations were measured in stormflow samples collected in the summer of 2017. Because the sampling equipment required modification, only a few samples were ultimately collected (two samples from LBDC, one from Hickey Run, three from Watts Branch, one from Fort DuPont Creek, and one from Pope Branch). Watts Branch was sampled on successive days (July 22 and 23) during the same storm event, and because of power failures, suspended sediment was not collected from NEB and NWB. In the fall of 2017, the focus of the fieldwork returned to collecting samples for organic COCs during storm events. Because of the limited number of stormflow samples collected in the summer, it was decided to aggregate the suspended-sediment and bed-sediment data to obtain a representative average concentration for each stream. Similar concentrations were found in the suspended sediment and bed sediment from NEB, LDBC, Hickey Run, and Watts Branch.

The following discussion focuses on the four metals of concern to the Anacostia River remediation: arsenic, copper, lead, and zinc; mercury was also considered as a metal of

Table 29. Total maximum loads of pesticides from all gaged tributaries to the Anacostia River, 2017.

[g/yr, grams per year; g/d, grams per day; kg/yr, kilograms per year; ND, compound not detected]

\begin{tabular}{lcc}
\hline $\begin{array}{c}\text { Sediment, pesticide, and } \\
\text { total organic carbon }\end{array}$ & \multicolumn{2}{c}{ All tributaries, 2017 } \\
\cline { 2 - 3 } Sediment load & $\mathbf{( g / y r )}$ & $\mathbf{( g / d )}$ \\
4,4'-DDD & $3.12 \times 10^{7}$ & 85,000 \\
4,4'-DDE & 28 & 0.075 \\
4,4'-DDT & 30 & 0.081 \\
Aldrin & 140 & 0.39 \\
alpha-BHC & 62 & 0.17 \\
beta-BHC & $\mathrm{ND}$ & $\mathrm{ND}$ \\
Chlordane & $\mathrm{ND}$ & $\mathrm{ND}$ \\
delta-BHC & 1,100 & 3.2 \\
Dieldrin & 14 & 0.041 \\
Endosulfan I & 870 & 2.4 \\
Endosulfan II & $\mathrm{ND}$ & $\mathrm{ND}$ \\
Endosulfan sulfate & $\mathrm{ND}$ & $\mathrm{ND}$ \\
Endrin & $\mathrm{ND}$ & $\mathrm{ND}$ \\
Endrin aldehyde & 0.59 & 0.001 \\
Endrin ketone & 200 & 0.56 \\
gamma-BHC (Lindane) & $\mathrm{ND}$ & $\mathrm{ND}$ \\
Heptachlor & 1.7 & 0.005 \\
Heptachlor epoxide & 5.5 & 0.016 \\
Total organic carbon ${ }^{1}$ & 42 & 0.12 \\
Toxaphene & $2.9 \times 10^{5}$ & 820 \\
Methoxychlor & $\mathrm{ND}$ & $\mathrm{ND}$ \\
\hline & 280 & 0.78 \\
\hline 'Sedin and & &
\end{tabular}

${ }^{1}$ Sediment and total organic carbon loads in $\mathrm{kg} / \mathrm{yr}$. concern in many urban rivers. Average concentrations of these metals are presented in table 30.

The highest concentrations of each metal are as follows: arsenic, $6.2 \mathrm{mg} / \mathrm{kg}$ in Watts Branch; copper, $210 \mathrm{mg} / \mathrm{kg}$ in LBDC; lead, $140 \mathrm{mg} / \mathrm{kg}$ in LBDC; zinc, $620 \mathrm{mg} / \mathrm{kg}$ in LBDC; and mercury, $0.33 \mathrm{mg} / \mathrm{kg}$ in LBDC.

By using the average concentrations in the aggregated dataset and the yearly sediment loads, the yearly loads of metals were calculated (table 31). Loads of arsenic in the gaged tributaries ranged from $0.74 \mathrm{~kg} / \mathrm{yr}(740 \mathrm{~g})$ in Hickey Run to $20 \mathrm{~kg} / \mathrm{yr}$ in LBDC; loads of copper ranged from $20 \mathrm{~kg} / \mathrm{yr}$ in Hickey Run to $920 \mathrm{~kg} / \mathrm{yr}$ in LBDC; loads of lead ranged from $18 \mathrm{~kg} / \mathrm{yr}$ in Hickey Run to $600 \mathrm{~kg} / \mathrm{yr}$ in LBDC; loads of zinc ranged from $39 \mathrm{~kg} / \mathrm{yr}$ in Hickey Run to 2,800 kg/yr in LBDC; and loads of mercury ranged from $0.009 \mathrm{~kg} / \mathrm{yr}(9.0 \mathrm{~g} / \mathrm{yr})$ in Hickey Run to $1.5 \mathrm{~kg}$ in LBDC. These data indicate that LBDC is the major source of the trace metals of concern for the Anacostia River. Although the total discharge and sediment load in LBDC $\left(5,100 \mathrm{Mgal}\right.$ and $4.64 \times 10^{6} \mathrm{~kg}$, respectively) (table 22) were about 30 percent of those in NEB and NWB (the largest sources of water and sediment to the Anacostia River), LBDC contributes the largest loading of these metals.

Table 30. Average concentrations of selected metals in suspended sediment from tributaries to the Anacostia River, 2017.

$[\mathrm{mg} / \mathrm{kg}$, milligrams per kilogram]

\begin{tabular}{lccccc}
\hline \multirow{2}{*}{ Tributary } & \multicolumn{5}{c}{ Average concentration (mg/kg) } \\
\cline { 2 - 6 } & Arsenic & Copper & Lead & Zinc & Mercury \\
\hline Northeast Branch & 0.70 & 4.8 & 4.1 & 21 & 0.008 \\
Northwest Branch & 0.63 & 8.0 & 9.4 & 20 & 0.0052 \\
Beaverdam Creek & 4.4 & 210 & 140 & 620 & 0.33 \\
Watts Branch & 6.2 & 51 & 81 & 230 & 0.096 \\
Hickey Run & 2.6 & 70 & 65 & 140 & 0.030 \\
Nash Run & 2.6 & 5.6 & 7.3 & 26 & 0.019 \\
Pope Branch & 4.2 & 15 & 16 & 160 & 0.021 \\
Fort DuPont Creek & 3.5 & 11 & 16 & 37 & 0.060 \\
Fort Stanton Creek & 5.9 & 10 & 8.9 & 42 & 0.0096 \\
\hline
\end{tabular}


Table 31. Loads of suspended-sediment-bound metals in tributaries to the Anacostia River, 2017.

[ $\mathrm{kg} / \mathrm{yr}$, kilograms per year; $\mathrm{kg} / \mathrm{d}$, kilograms per day; $<$, less than]

\begin{tabular}{|c|c|c|c|c|c|c|c|c|c|c|}
\hline \multirow{2}{*}{ Metal } & \multicolumn{2}{|c|}{ Northeast Branch } & \multicolumn{2}{|c|}{ Northwest Branch } & \multicolumn{2}{|c|}{ Beaverdam Creek } & \multicolumn{2}{|c|}{ Watts Branch } & \multicolumn{2}{|c|}{ Hickey Run } \\
\hline & (kg/yr) & $(\mathrm{kg} / \mathrm{d})$ & $(\mathrm{kg} / \mathrm{yr})$ & $(\mathrm{kg} / \mathrm{yr})$ & $(\mathrm{kg} / \mathrm{yr})$ & $(\mathrm{kg} / \mathrm{d})$ & $(\mathrm{kg} / \mathrm{yr})$ & $(\mathrm{kg} / \mathrm{d})$ & $(\mathrm{kg} / \mathrm{yr})$ & $(\mathrm{kg} / \mathrm{yr})$ \\
\hline Sediment & $1.02 \times 10^{7}$ & 27,900 & $1.55 \times 10^{7}$ & 42,300 & $4.45 \times 10^{6}$ & 12,200 & $6.52 \times 10^{5}$ & 1,550 & $2.82 \times 10^{5}$ & 1,290 \\
\hline Aluminum & 8,000 & 22 & 32,000 & 86 & 17,000 & 47 & 3,100 & 8.5 & 940 & 4.3 \\
\hline Antimony & 0.84 & 0.002 & 0.96 & 0.003 & 17 & 0.047 & 0.72 & 0.002 & 0.33 & 0.001 \\
\hline Arsenic & 7.1 & 0.019 & 9.7 & 0.027 & 20 & 0.054 & 3.5 & 0.010 & 0.74 & 0.003 \\
\hline Barium & 84 & 0.23 & 220 & 0.59 & 360 & 0.97 & 44 & 0.12 & 15 & 0.069 \\
\hline Beryllium & 1.9 & 0.005 & 3.7 & 0.010 & 2.5 & 0.007 & 0.51 & 0.001 & 0.084 & $<0.001$ \\
\hline Cadmium & 0.49 & 0.001 & 0.77 & 0.002 & 6.7 & 0.018 & 0.64 & 0.002 & 0.082 & $<0.001$ \\
\hline Calcium & 36,000 & 98 & 12,000 & 33 & 17,000 & 48 & 2,500 & 7.0 & 7,400 & 34 \\
\hline Chromium & 61 & 0.17 & 170 & 0.47 & 100 & 0.28 & 13 & 0.037 & 7.6 & 0.035 \\
\hline Cobalt & 31 & 0.085 & 51 & 0.14 & 50 & 0.14 & 14 & 0.038 & 1.7 & 0.008 \\
\hline Copper & 49 & 0.13 & 120 & 0.34 & 920 & 2.5 & 29 & 0.080 & 20 & 0.091 \\
\hline Iron & 50,000 & 140 & 98,000 & 270 & 77,000 & 210 & 13,000 & 35 & 4,500 & 21 \\
\hline Lead & 41 & 0.11 & 150 & 0.40 & 600 & 1.6 & 46 & 0.13 & 18 & 0.083 \\
\hline Magnesium & 20,000 & 54 & 26,000 & 72 & 9,600 & 26 & 1,400 & 3.9 & 3,400 & 16 \\
\hline Manganese & 1,000 & 2.8 & 1,200 & 3.2 & 990 & 2.7 & 290 & 0.79 & 87 & 0.40 \\
\hline Mercury & 0.08 & $<0.001$ & 0.08 & $<0.001$ & 1.5 & 0.004 & 0.054 & $<0.001$ & 0.009 & $<0.001$ \\
\hline Nickel & 53 & 0.14 & 180 & 0.48 & 110 & 0.30 & 19 & 0.052 & 8.1 & 0.037 \\
\hline Potassium & 1,300 & 3.6 & 7,600 & 21 & 2,600 & 7.2 & 440 & 1.2 & 100 & 0.47 \\
\hline Selenium & 1.1 & 0.003 & 2.5 & 0.007 & 1.9 & 0.005 & 0.54 & 0.001 & 0.070 & $<0.001$ \\
\hline Silver & 0.17 & $<0.001$ & 0.22 & 0.001 & 2.7 & 0.007 & 0.13 & $<0.001$ & 0.031 & $<0.001$ \\
\hline Sodium & 400 & 1.1 & 840 & 2.3 & 540 & 1.5 & 73 & 0.20 & 64 & 0.29 \\
\hline Thallium & 0.17 & 0.000 & 0.74 & 0.002 & 0.32 & 0.001 & 0.082 & $<0.001$ & 0.017 & $<0.001$ \\
\hline Vanadium & 61 & 0.17 & 120 & 0.32 & 99 & 0.27 & 17 & 0.047 & 7.0 & 0.032 \\
\hline Zinc & 214 & 0.59 & 310 & 0.85 & 2,800 & 7.6 & 130 & 0.35 & 39 & 0.18 \\
\hline
\end{tabular}


Table 31. Loads of suspended-sediment bound-metals in tributaries to the Anacostia River, 2017._Continued $[\mathrm{kg} / \mathrm{yr}$, kilograms per year; $\mathrm{kg} / \mathrm{d}$, kilograms per day; <, less than]

\begin{tabular}{|c|c|c|c|c|c|c|c|c|}
\hline \multirow{2}{*}{ Metal } & \multicolumn{2}{|c|}{ Nash Run } & \multicolumn{2}{|c|}{ Pope Branch } & \multicolumn{2}{|c|}{ Fort DuPont Creek } & \multicolumn{2}{|c|}{ Fort Stanton Creek } \\
\hline & (kg/yr) & $(\mathrm{kg} / \mathrm{d})$ & $(\mathrm{kg} / \mathrm{yr})$ & $(\mathrm{kg} / \mathrm{d})$ & (kg/yr) & $(\mathrm{kg} / \mathrm{d})$ & (kg/yr) & $(\mathrm{kg} / \mathrm{d})$ \\
\hline Sediment & 1.2 & 323 & $6.18 \times 10^{4}$ & 169 & $1.12 \times 10^{5}$ & 307 & $5.62 \times 10^{4}$ & 154 \\
\hline Aluminum & 120 & 0.32 & 130 & 0.35 & 270 & 0.74 & 96 & 0.26 \\
\hline Antimony & 0.013 & $<0.001$ & 0.071 & $<0.001$ & 0.032 & $<0.001$ & 0.013 & $<0.001$ \\
\hline Arsenic & 0.31 & 0.001 & 0.26 & 0.001 & 0.39 & 0.001 & 0.33 & 0.001 \\
\hline Barium & 1.3 & 0.004 & 1.9 & 0.005 & 4.4 & 0.012 & 1.1 & 0.003 \\
\hline Beryllium & 0.057 & $<0.001$ & 0.014 & $<0.001$ & 0.057 & $<0.001$ & 0.036 & $<0.001$ \\
\hline Cadmium & 0.007 & $<0.001$ & 0.011 & $<0.001$ & 0.014 & $<0.001$ & 0.011 & $<0.001$ \\
\hline Calcium & 39. & 0.11 & 120 & 0.34 & 92. & 0.253 & 27 & 0.074 \\
\hline Chromium & 0.80 & 0.002 & 0.99 & 0.003 & 1.4 & 0.004 & 1.3 & 0.004 \\
\hline Cobalt & 0.78 & 0.002 & 0.52 & 0.001 & 1.1 & 0.003 & 0.28 & 0.001 \\
\hline Copper & 0.66 & 0.002 & 0.92 & 0.003 & 1.3 & 0.003 & 0.56 & 0.002 \\
\hline Iron & 1,400 & 3.9 & 1,200 & 3.4 & 1,800 & 4.9 & 1,900 & 5.2 \\
\hline Lead & 0.86 & 0.002 & 0.99 & 0.003 & 1.8 & 0.005 & 0.50 & 0.001 \\
\hline Magnesium & 59 & 0.16 & 550 & 1.5 & 45 & 0.12 & 12 & 0.032 \\
\hline Manganese & 14 & 0.039 & 49 & 0.13 & 28 & 0.078 & 13 & 0.037 \\
\hline Mercury & 0.002 & $<0.001$ & 0.001 & $<0.001$ & 0.0070 & $<0.001$ & 0.001 & $<0.001$ \\
\hline Nickel & 1.1 & 0.003 & 6.7 & 0.018 & 0.87 & 0.002 & 0.34 & 0.001 \\
\hline Potassium & 11 & 0.031 & 17 & 0.046 & 33 & 0.090 & 8.4 & 0.023 \\
\hline Selenium & 0.025 & $<0.001$ & 0.037 & $<0.001$ & 0.055 & 0.000 & 0.023 & $<0.001$ \\
\hline Silver & 0.002 & $<0.001$ & 0.004 & $<0.001$ & 0.030 & $<0.001$ & 0.001 & $<0.001$ \\
\hline Sodium & 3.9 & 0.011 & 6.5 & 0.018 & 4.4 & 0.012 & 1.5 & 0.004 \\
\hline Thallium & 0.003 & $<0.001$ & 0.002 & $<0.001$ & 0.006 & $<0.001$ & 0.001 & $<0.001$ \\
\hline Vanadium & 1.7 & 0.005 & 0.90 & 0.002 & 2.4 & 0.006 & 3.8 & 0.010 \\
\hline Zinc & 3.1 & 0.008 & 9.9 & 0.027 & 4.1 & 0.011 & 2.4 & 0.006 \\
\hline
\end{tabular}




\section{Comparison of Loads of Contaminants of Concern with Total Maximum Daily Load Allocations}

Total maximum daily load (TMDL) allocations were promulgated by the EPA in 2003 for 16 pollutants in 13 segments of streams within the Anacostia River watershed (D.C. Department of Health, 2003; U.S. Environmental Protection Agency, 2003). TMDLs are defined as the allowable permissible discharge to a water body from point sources (such as combined sewer overflows and stormwater outfalls), nonpoint sources (such as the atmosphere), plus a safety margin. Load allocations are typically based on average concentrations measured (or in some cases assumed) in stormwater and base-flow samples and discharges either measured at gaging stations or derived from hydrodynamic and stormwater-routing models. Point sources such as stormwater and other discharges to tributaries are regulated through EPA National Pollutant Discharge Elimination System (NPDES) permits. The TMDL allocations represent the allowable loadings to the tributaries that are predicted by means of model simulations to ultimately reduce contaminant concentrations to meet water-quality standards.

The number of pollutants for which TMDLs were set ranged from 3 in Fort DuPont Creek to 16 in the NEB and NWB (collectively identified as the upper Anacostia River). A subset of the constituents having TMDLs overlaps with constituents measured in this study and includes total suspended sediment, three groups of PCBs and PAHs, and three metals. PCB congeners are grouped into homologs; the PCB-1 group includes the di- and tri-chlorinated homologs, the PCB-2 group includes the tetra- through hexa-homologs, and the PCB-3 group includes the hepta- through nona-homologs. The first group of PAHs (PAH-1) includes naphthalene, 2-methylnaphthalene, acenaphthylene, fluorene, and phenanthrene; the second group (PAH-2) includes fluoranthene, pyrene, benzo[a]anthracene, and chrysene; and the third group (PAH-3) includes benzo(k)fluoranthene, benzo(a)pyrene, perylene, indeno(1,2,3-c,d), pyrene, benzo(g,h,i)perylene, and dibenzo $(\mathrm{a}, \mathrm{h}+\mathrm{ac})$ anthracene. TMDLs were also set for chlordane, heptachlor epoxide, dieldrin, DDT, DDE, and DDD, and for arsenic, copper, lead, and zinc.

The loadings listed below were taken from the Anacostia River Implementation Plan (Washington, D.C., Department of Energy \& Environment, 2012) and were converted to units of grams per year. The TMDLs split the allocations into the Maryland and Washington, D.C. (D.C.), contributions on the basis of the percentage of basin area within each jurisdiction. The allocations for NEB and NWB were attributed to Maryland as a combined loading for the two mainstem tributaries. Watts Branch was divided into a Maryland component and a D.C. component, whereas that for LBDC was attributed entirely to Maryland. Allocations for Hickey Run, Nash Run, and the other small tributaries were attributed solely to D.C.

The data from the current study were regrouped as needed to compare them with the TMDLs. The average concentrations of individual PAHs (app. 2, table 2.3) were grouped into PAH subgroups, and the PCBs were grouped by homologs; these results are presented in table 32 . The calculated loadings for 2017 are compared with the TMDL allocations in table 33.

In 2017, LBDC exceeded the load allocations for the PCB-2 group and for total PCBs. Almost all the tributaries exceeded the limit for the three PAH groups. The combined loadings of NEB and NWB exceeded the PAH allocations by a factor of nearly 200 , and LBDC exceeded its allocation by a factor of almost 100 . Watts Branch exceeded the PAH allocation by a factor of 15 and Hickey Run exceeded it by a factor of almost 4 . Of the small ungaged tributaries, Nash Run exceeded its PAH allocation by a factor of more than 2 .

The pesticide and metal loadings reported here are subject to uncertainty because they are based on a single sample and may not accurately represent the variation in concentrations that may exist. However, the current data indicate the 2017 loading for chlordane exceeded the TMDL by a factor of about 9 in the combined NEB and NWB tributaries, by a factor of nearly 16 in LBDC, by a factor of 20 in Watts Branch, and by a factor of nearly 2 in Hickey Run. For metals, only the load of copper in LBDC exceeded the TMDL allocation. 
Table 32. Average concentrations of selected constituents in tributaries to the Anacostia River grouped into categories.

[ $\mu \mathrm{g} / \mathrm{kg}$, micrograms per kilograms; mg/kg, milligrams per kilogram; nd, not detected; PCB, polychlorinated biphenyl; PAH, polycyclic aromatic hydrocarbon]

\begin{tabular}{|c|c|c|c|c|c|c|}
\hline Constituent & $\begin{array}{l}\text { Northeast } \\
\text { Branch }\end{array}$ & $\begin{array}{c}\text { Northwest } \\
\text { Branch }\end{array}$ & $\begin{array}{c}\text { Beaverdam } \\
\text { Creek }\end{array}$ & $\begin{array}{l}\text { Watts } \\
\text { Branch }\end{array}$ & $\begin{array}{c}\text { Hickey } \\
\text { Run }\end{array}$ & $\begin{array}{l}\text { Nash } \\
\text { Run }\end{array}$ \\
\hline \multicolumn{7}{|c|}{ PCB Homolog Group 1} \\
\hline Dichloro-PCBs $(\mu \mathrm{g} / \mathrm{kg})$ & 0.22 & 0.33 & 5.3 & 0.44 & 0.82 & 2.0 \\
\hline Total PCB Group $1(\mu \mathrm{g} / \mathrm{kg})$ & 0.37 & 0.54 & 29 & 1.3 & 2.2 & 6.4 \\
\hline \multicolumn{7}{|c|}{ PCB Homolog Group 2} \\
\hline Tetra-PCBs $(\mu \mathrm{g} / \mathrm{kg})$ & 0.32 & 0.39 & 39 & 5.1 & 4.5 & 8.9 \\
\hline Total PCB Group $2(\mu \mathrm{g} / \mathrm{kg})$ & 3.4 & 3.6 & 87 & 39 & 49 & 49 \\
\hline \multicolumn{7}{|c|}{ PCB Homolog Group 3} \\
\hline Hepta-PCBs $(\mu \mathrm{g} / \mathrm{kg})$ & 1.6 & 2.3 & 12 & 2.9 & 13 & 6.1 \\
\hline Octa-PCBs $(\mu \mathrm{g} / \mathrm{kg})$ & 0.42 & 0.22 & 3.1 & 0.62 & 3.6 & 1.7 \\
\hline Nona-PCBs $(\mu \mathrm{g} / \mathrm{kg})$ & 0.075 & 0.19 & 0.43 & 0.22 & 0.5 & 1.2 \\
\hline \multicolumn{7}{|c|}{ PAH Group 1} \\
\hline 2-Methylnaphthalene $(\mu \mathrm{g} / \mathrm{kg})$ & 6.5 & 7.7 & 9.9 & 5.3 & 39 & 18 \\
\hline Acenaphthylene $(\mu \mathrm{g} / \mathrm{kg})$ & 3.0 & 4.5 & 3.9 & 7.1 & 17 & 7.1 \\
\hline Acenaphthene $(\mu \mathrm{g} / \mathrm{kg})$ & 4.7 & 6.2 & 5.0 & 5.0 & 23 & 18 \\
\hline Fluorene $(\mu \mathrm{g} / \mathrm{kg})$ & 6.9 & 8.6 & 7.4 & 7.4 & 58 & 14 \\
\hline Phenanthrene $(\mu \mathrm{g} / \mathrm{kg})$ & 110 & 146 & 96 & 90 & 440 & 190 \\
\hline Total PAH Group $1(\mu \mathrm{g} / \mathrm{kg})$ & 140 & 190 & 140 & 130 & 620 & 250 \\
\hline \multicolumn{7}{|c|}{ PAH Group 2} \\
\hline Fluoranthene $(\mu \mathrm{g} / \mathrm{kg})$ & 236 & 320 & 220 & 200 & 550 & 410 \\
\hline Pyrene $(\mu \mathrm{g} / \mathrm{kg})$ & 176 & 200 & 220 & 190 & 1,120 & 360 \\
\hline Benzo(a)anthracene $(\mu \mathrm{g} / \mathrm{kg})$ & 76 & 110 & 91 & 95 & 290 & 220 \\
\hline Chrysene $(\mu \mathrm{g} / \mathrm{kg})$ & 170 & 270 & 170 & 170 & 550 & 330 \\
\hline Dibenzo(a,h+ac)anthracene $(\mu \mathrm{g} / \mathrm{kg})$ & 20 & 31 & 20 & 20 & 52 & 40 \\
\hline Total PAH Group $3(\mu \mathrm{g} / \mathrm{kg})$ & 430 & 630 & 460 & 430 & 1,200 & 900 \\
\hline Total PAHs $(\mu \mathrm{g} / \mathrm{kg})$ & 1,200 & 1,700 & 1,300 & 1,200 & 4,400 & 2,500 \\
\hline \multicolumn{7}{|c|}{ Pesticides $^{1}$} \\
\hline Chlordane $(\mu \mathrm{g} / \mathrm{kg})$ & 30 & 3.4 & 43 & 62 & 27 & nd \\
\hline 4,4'-DDD ( $\mu \mathrm{g} / \mathrm{kg})$ & 0.79 & 0.29 & 2.2 & 1.4 & 2.9 & 0.23 \\
\hline 4,4'-DDE $(\mu \mathrm{g} / \mathrm{kg})$ & 0.25 & 1.1 & 1.5 & 1.9 & 3.5 & nd \\
\hline 4,4'-DDT $(\mu \mathrm{g} / \mathrm{kg})$ & nd & 1.5 & 2.0 & 37 & 7.6 & nd \\
\hline Dieldrin $(\mu \mathrm{g} / \mathrm{kg})$ & 0.34 & 14 & 0.39 & 0.44 & 0.68 & 0.041 \\
\hline \multicolumn{7}{|c|}{ Metals } \\
\hline Arsenic (mg/kg) & 0.7 & 0.63 & 11 & 11 & 4.5 & 2.6 \\
\hline Copper (mg/kg) & 4.8 & 8.0 & 690 & 92 & 110 & 5.6 \\
\hline Lead $(\mathrm{mg} / \mathrm{kg})$ & 4.1 & 9.4 & 430 & 100 & 130 & 7.3 \\
\hline Zinc $(\mathrm{mg} / \mathrm{kg})$ & 21 & 20 & 2,100 & 410 & 540 & 26 \\
\hline Mercury (mg/kg) & 0.0079 & 0.0052 & 1.1 & 0.18 & 0.14 & 0.019 \\
\hline
\end{tabular}

${ }^{1}$ Values reported for pesticides are the maximum concentration in the combined set of suspended-sediment and bed-sediment analyses. 
Table 33. Loadings of selected constituents in tributaries to the Anacostia River compared with permitted total maximum daily load allocations.

[NEB, Northeast Branch; NWB, Northwest Branch; TMDL, total maximum daily load; PCB, polychlorinated biphenyl; PAH, polycyclic aromatic hydrocarbon; g/yr, grams per year; kg/yr, kilograms per year; nd, not detected; NA, not applicable, the permitted allocations are not set]

\begin{tabular}{|c|c|c|c|c|c|c|c|c|c|c|c|c|}
\hline Constituent & NEB & NWB & NEB + NWB & $\begin{array}{c}\text { TMDL } \\
\text { allotment }^{1}\end{array}$ & $\begin{array}{c}\text { Beaverdam } \\
\text { Creek }\end{array}$ & $\begin{array}{c}\text { TMDL } \\
\text { allotment }^{1}\end{array}$ & $\begin{array}{c}\text { Watts } \\
\text { Branch }\end{array}$ & $\begin{array}{c}\text { TMDL } \\
\text { allotment }^{1}\end{array}$ & $\begin{array}{c}\text { Hickey } \\
\text { Run }\end{array}$ & $\begin{array}{c}\text { TMDL } \\
\text { allotment }^{1}\end{array}$ & $\begin{array}{l}\text { Nash } \\
\text { Run }\end{array}$ & $\begin{array}{c}\text { TMDL } \\
\text { allotment }^{1}\end{array}$ \\
\hline \multicolumn{13}{|c|}{ PCBs } \\
\hline PCB Group 1 (g/yr) & 3.7 & 8.3 & 12 & 69 & 130 & 140 & 0.75 & 21 & 0.62 & NA & 0.75 & NA \\
\hline PCB Group 2 (g/yr) & 35 & 56 & 91 & 670 & 390 & 280 & 22 & 42 & 14 & NA & 5.8 & NA \\
\hline PCB Group 3 (g/yr) & 21 & 42 & 63 & 490 & 69 & 77 & 2.1 & 12 & 4.9 & NA & 1.1 & NA \\
\hline PCBs $(g / y r)$ & 60 & 110 & 170 & 1,200 & 590 & 500 & 25 & 75 & 19 & 295 & 7.6 & 130 \\
\hline \multicolumn{13}{|c|}{ PAHs } \\
\hline PAH Group 1 (kg/yr) & 15 & 29 & 44 & 0.21 & 6.3 & 0.055 & 0.80 & 0.41 & 1.7 & 3.0 & 0.29 & 1.2 \\
\hline PAH Group 2 (kg/yr) & 67 & 140 & 210 & 1.2 & 31 & 0.34 & 3.6 & 0.42 & 7.1 & 0.35 & 1.6 & 1.4 \\
\hline PAH Group 3 (kg/yr) & 44 & 100 & 140 & 0.76 & 20 & 0.22 & 2.4 & 0.16 & 3.4 & 0.230 & 1.1 & 0.091 \\
\hline PAH (kg/yr) & 130 & 270 & 400 & 2.2 & 59 & 0.62 & 6.8 & 0.44 & 12 & 3.5 & 2.9 & 1.4 \\
\hline \multicolumn{13}{|c|}{ Pesticides $^{2}$} \\
\hline Chlordane (g/yr) & 430 & 320 & 750 & 83 & 310 & 20 & 56 & 3.0 & 11 & 6.5 & ND & 2.6 \\
\hline 4,4'-DDD (g/yr) & 8.1 & 7.1 & 15 & 100 & 9.8 & 19 & 1.0 & 4.7 & 1.3 & 2.4 & 0.027 & 1.0 \\
\hline 4,4'-DDE (g/yr) & 2.6 & 17 & 20 & 22 & 6.7 & 71 & 1.5 & 12 & 1.6 & 5.2 & ND & 2.1 \\
\hline 4,4'-DDT (g/yr) & ND & 49 & 49 & 13 & 8.9 & 9.2 & 79 & 1.6 & 6.5 & 0.65 & ND & 2.1 \\
\hline Dieldrin (g/yr) & 4.7 & 860 & 860 & 18 & 1.7 & 1.6 & 0.34 & 0.44 & 0.25 & 0.57 & 0.005 & 2.1 \\
\hline \multicolumn{13}{|c|}{ Metals } \\
\hline Arsenic (kg/yr) & 7.1 & 9.7 & 17 & 18 & 20 & 4,400 & 3.5 & 660 & 0.74 & NA & 0.31 & 350 \\
\hline Copper (kg/yr) & 49 & 120 & 170 & 2,700 & 920 & 490 & 29 & 170 & 20 & NA & 0.66 & 24,000 \\
\hline Lead (kg/yr) & 41 & 150 & 190 & 2,700 & 600 & 720 & 46 & 83 & 18 & NA & 0.86 & 9,000 \\
\hline Zinc (kg/yr) & 210 & 310 & 520 & 6,900 & 2,800 & 3,600 & 130 & 500 & 39 & NA & 3.1 & 180 \\
\hline
\end{tabular}

Zinc $(\mathrm{kg} / \mathrm{yr})$

${ }^{1}$ Loadings are the sum of the total allocation allowed for Maryland and Washington, D.C. Shaded values indicate exceedance of permitted allocations.

${ }^{2}$ Pesticide loadings represent maximum loadings calculated from maximum concentrations in the combined set of suspended-sediment and bed-sediment analyses. 


\section{Summary}

A study was undertaken by the U.S. Geological Survey in cooperation with the Washington, D.C., Department of Energy \& Environment to estimate concentrations and loads of sediment-bound polychlorinated biphenyls (PCBs), polycyclic aromatic hydrocarbons (PAHs), organochlorine pesticides, and trace metals in tributaries to the Anacostia River (known locally as "Lower Anacostia River") for the 2017 calendar year. These tributaries include the gaged streams Northeast Branch of the Anacostia River (NEB) and Northwest Branch of the Anacostia River (NWB), Beaverdam Creek (known locally as "Lower Beaverdam Creek") (LBDC), Watts Branch, and Hickey Run, and the ungaged streams Nash Run, Pope Branch, an unnamed tributary at Fort DuPont (called "Fort DuPont Creek" in this report), and an unnamed tributary at Fort Stanton (called "Fort Stanton Creek" in this report). The basins of these tributaries vary greatly in area, but all are highly urbanized and receive input from extensive stormwatercollection systems.

Chemical data were obtained from large-volume composite samples of streamwater and suspended sediment collected during four to five storms events and one to two times during periods of low flow. Sediment was analyzed for 209 PCB congeners (109 unique congeners or coelutions), 35 PAHs (20 nonalkylated and 15 alkylated compounds), 20 organochlorine pesticides, and 23 metals (in one storm sample only). Models were developed relating suspended-sediment concentrations (SSCs) measured in discrete grab samples to turbidity and discharge. SSC was then estimated by using continuous turbidity and discharge measurements made in these tributaries. Sediment loadings in the four small ungaged tributaries were estimated from the Watts Branch loading, adjusted for individual basin area. This approach is appropriate because of the proximity and similarity of the ungaged basins to the Watts Branch Basin.

For 2017, the total sediment loading from these tributaries to the Anacostia River was $3.10 \times 10^{7}$ kilograms $(\mathrm{kg})$, with 50 percent originating from NWB, 33 percent from NEB, 14 percent from LBDC, and less than 2 percent each from Watts Branch and Hickey Run. The high sediment loading from NEB and NWB is consistent with their large basin areas and discharges. Sediment yields ranged from $1.40 \times 10^{5}$ kilograms per year per square mile $\left(\mathrm{kg} / \mathrm{yr} / \mathrm{mi}^{2}\right)$ for NEB to $3.13 \times 10^{5} \mathrm{~kg} / \mathrm{yr} / \mathrm{mi}^{2}$ for NWB. The yield calculated for LBDC $\left(3.01 \times 10^{5} \mathrm{~kg} / \mathrm{yr} / \mathrm{mi}^{2}\right)$ nearly matched that for NWB and is out of proportion to its basin area. The smaller ungaged tributaries supplied only a minimal amount of sediment $\left(3.5 \times 10^{5} \mathrm{~kg}\right)$, equal to about 1 percent of the total sediment from the gaged tributaries. However, as a result of gaps in turbidity and discharge data, the sediment (and therefore chemical) loads reported for LBDC are considered to be underestimated. Data records for all other gaged tributaries (once gaps were filled with estimated values) covered 100 percent of the year.

Concentrations of PCBs and PAHs were measurable in all suspended-sediment samples collected from the gaged and ungaged tributaries. Absolute concentrations were highest in low-flow samples and lowest in stormflow samples; concentrations in bed sediment were intermediate. This observation is consistent with the fact that only the finest grained materials are transported during low-flow conditions. Comparison of molar PCB percentages showed that sediment contaminant makeup was similar among the three types of sediment collected, supporting the use of an average concentration to determine loadings in each stream. Average total PCB concentrations were highest in samples from LBDC (130 micrograms per kilogram $[\mu \mathrm{g} / \mathrm{kg}])$, followed by Hickey Run $(69 \mu \mathrm{g} / \mathrm{kg})$ and Watts Branch $(44 \mu \mathrm{g} / \mathrm{kg})$. Total PCB concentrations in samples from NWB $(6.6 \mu \mathrm{g} / \mathrm{kg})$ and NEB $(5.9 \mu \mathrm{g} / \mathrm{kg})$ were an order of magnitude smaller than those in samples from the other gaged tributaries. PCB congener profiles in samples from the gaged tributaries except LBDC were similar, being dominated by the penta- through octa-homologs. In LBDC sediment, the PCB profiles were dominated by the less chlorinated congeners from the mono- through penta-homologs, indicating that the source of the PCBs in LBDC is different from the source of the PCBs in the other tributaries.

By using the average concentrations determined in the combined storm and low-flow datasets, a total of 820 grams (g) of PCBs was supplied to the Anacostia River by the tributaries in 2017, with 75 percent originating from LBDC, 12 percent from NWB, and 7 percent from NEB, with less than 3 percent each originating from Watts Branch and Hickey Run. The load of total PAHs, defined as the sum of all nonalkylated and alkylated species, was 89,000 g, with NWB accounting for 59 percent, NEB contributing 23 percent, LBDC supplying 11 percent, Hickey Run supplying 6 percent, and Watts Branch contributing less than 2 percent. Total nonalkylated PAH loading was 64,000 g, with NWB and NEB supplying most (59 and 25 percent of the total, respectively) of the load. The ungaged tributaries contributed $8.1 \mathrm{~g}$ of total PCBs and $770 \mathrm{~g}$ of total PAHs, with Nash Run providing most of the PCBs $(7.6 \mathrm{~g})$ and PAHs $(650 \mathrm{~g})$.

These results show that in 2017, LBDC was the largest source of PCBs to the Anacostia River, whereas NWB was the largest source of PAHs. The ungaged tributaries provided extremely small amounts of contaminants of concern (COCs), with Nash Run dominating the load contributions from the ungaged streams.

Pesticide analysis was complicated by the differences in minimum detection levels (MDLs) among low-flow, stormflow, and bed-sediment samples. Additionally, because sampling for pesticides (and metals) was begun in the summer of 2017, only a few (one to three) samples were obtained from each tributary. The elevated MDLs resulted in unquantifiable concentrations for many of the pesticides in the suspendedsediment samples; the exception was chlordane, which was found in quantifiable concentrations in all samples of suspended sediment from the gaged streams. Consequently, loadings for individual pesticides were calculated by using the maximum concentration in the combined suspended-sediment and bed-sediment samples from each tributary. The highest 
average chlordane concentration was $100 \mu \mathrm{g} / \mathrm{kg}$ measured in Watts Branch. Chlordane loading for all tributaries totaled $1,100 \mathrm{~g}$, with 37 percent ( $430 \mathrm{~g}$ ) supplied by NEB, 28 percent $(320 \mathrm{~g})$ contributed by NWB, 28 percent $(310 \mathrm{~g})$ originating from LBDC, 5 percent $(56 \mathrm{~g}$ ) supplied by Watts Branch, and 1 percent $(11 \mathrm{~g})$ contributed by Hickey Run. Chlordane was not present at quantifiable levels in any of the sediment samples collected from the ungaged tributaries. These results indicate that chlordane is supplied principally by the NEB, NWB, and LBDC tributaries. LBDC supplied nearly the same mass of chlordane as did NWB, although NWB supplied considerably more sediment (3.5 times). The freshly deposited bed sediment showed the presence of other pesticides in these streams, including dieldrin, methoxychlor, endrin aldehyde, and 4,4'-DDT, indicating that these tributaries may contribute substantial loads of these pesticides. Additional sampling and modification of analytical methods will be needed to confirm these pesticide loads.

Overall, these results indicate that, in 2017, substantial loadings of PCBs, PAHs, and chlordane entered the Anacostia River from the gaged tributaries, whereas the loads supplied by the ungaged tributaries were negligible. The results from this study indicate that LBDC is a large source of COCs, much greater than would be expected from its basin area. Additional sampling will be needed to confirm and refine the concentrations and loadings of pesticides and metals in these tributaries. Additional sampling and analysis would help to identify the sources of COCs to LBDC, Watts Branch, Hickey Run, and Nash Run, as well as the sources of sediment in the NEB, NWB, and LBDC tributaries.

\section{References Cited}

Abdel-Shafy, H.-I., and Mansour, M.S.M., 2016, A review on polycyclic aromatic hydrocarbons-Source, environmental impact, effect on human health and remediation: Egyptian Journal of Petroleum, v. 25, no. 1, p. 107-123.

Anderson, C.W., 2005, Turbidity (ver. 2.1): U.S. Geological Survey Techniques of Water-Resources Investigations, book 9, chap. A6.7, https://doi.org/10.3133/twri09A6.7.

Andersson, J.T., and Achten, C., 2015, Time to say goodbye to the 16 EPA PAHs? Toward an up-to-date use of PACs for environmental purposes: Polycyclic Aromatic Compounds, v. 35 , no. $2-4$, p. $330-354$.

Baird, S.J.S., Bailey, E.A., and Vorhees, D.J., 2007, Evaluating human risk from exposure to alkylated PAHs in an aquatic system: Human and Ecological Risk Assessment, v. 13, no. 2, p. 322-338.

Cohn, T.A., DeLong, L.L., Gilroy, E.J., Hirsch, R.M., and Wells, D.K., 1989, Estimating constituent loads: Water Resources Research, v. 25, no. 5, p. 937-942.
D.C. Department of Health, 2003, District of Columbia Final Total Maximum Daily Loads for organics and metals in the Anacostia River, Fort Champlain Tributary, Fort Davis Tributary, Fort Dupont Creek, Fort Stanton Tributary, Hickey Run, Nash Run, Popes Branch, Texas Avenue Tributary, and Watts Branch: Washington, D.C., D.C. Department of Health, Environmental Health Administration, August 2003, 63 p., 6 app.

Erikson, M.D., 1997, Analytical chemistry of PCBs (2d ed.): New York, CRC Press, 688 p.

Flesher, J.W., and Lehner, A.F., 2016, Structure, function, and carcinogenicity of metabolites of methylated and non-methylated polycyclic aromatic hydrocarbons-A comprehensive review: Toxicology Mechanisms and Methods, v. 26, no. 3, p. 151-179.

Foster, G.D., Roberts, E.C., Jr., Gruessner, B., and Velinsky, D.J., 2000, Hydrogeochemistry and transport of organic contaminants in an urban watershed of Chesapeake Bay (USA): Applied Geochemistry, v. 15, no. 7, p. 901-915.

Helsel, D.R., and Hirsch, R.M., Statistical methods in water resources: Amsterdam, Elsevier, 529 p.

Hwang, H.-M., and Foster, G.D., 2008, Polychlorinated biphenyls in stormwater entering the tidal Anacostia River, Washington, DC, through small urban catchments and combined sewer outfalls: Journal of Environmental Science and Health, Part A, v. 43, no. 6, p. 567-575.

Jastram, J.D., Moyer, D.L., and Hyer, K.E., 2009, A comparison of turbidity-based and streamflow-based estimates of suspended-sediment concentrations in three Chesapeake Bay tributaries: U.S. Geological Survey Scientific Investigations Report 2009-5165, 37 p.

Kodavanti, P.R.S., Kannan, N., Yamashita, N., Derr-Yellin, E.C., Ward, T.R., Burgin, D.E., Tilson, H.A., and Birnbaum, L.S., 2001, Differential effects of two lots of aroclor 1254-Congener-specific analysis and neurochemical end points: Environmental Health Perspectives, v. 109, no. 11, p. $1153-1161$.

Levesque, V.A., and Oberg, K.A., 2012, Computing discharge using the index velocity method: U.S. Geological Survey Techniques and Methods, book 3, chap. A23, 148 p. (Also available at $h t t p s: / / p u b s . u s g s . g o v / t m / 3 a 23 /$.

Metropolitan Washington Council of Governments, 2000, Fort Dupont subwatershed restoration, 1999 baseline stream assessment study - Physical, chemical, and biological conditions: Washington, D.C., Metropolitan Washington Council of Governments, Department of Environmental Programs, prepared for U.S. Geological Survey, April 2000, $70 \mathrm{p}$. 
Metropolitan Washington Council of Governments, 2014, Draft technical memorandum-Watts Branch post-construction monitoring: Washington, D.C., Metropolitan Washington Council of Governments, Department of Environmental Programs, prepared for District Department of the Environment, Watershed Protection Division, November 2014, 53 p.

Miller, C.V., Chanat, J.G., and Bell, J.M., 2013, Water quality in the Anacostia River, Maryland and Rock Creek, Washington, D.C.-Continuous and discrete monitoring with simulations to estimate concentrations and yields of nutrients, suspended sediment, and bacteria: U.S. Geological Survey Open-File Report 2013-1034, 37 p.

Miller, C.V., Gutierrez-Magness, A.L., Feit Majedi, B.L., and Foster, G.D., 2007, Water quality in the Upper Anacostia River, Maryland - Continuous and discrete monitoring with simulations to estimate concentrations and yields, 2003-05: U.S. Geological Survey Scientific Investigations Report 2007-5142, 43 p.

Oberg, K.A., Morlock, S.E., and Caldwell, W.S., 2005, Quality assurance plan for discharge measurements using acoustic doppler current profilers: U.S. Geological Survey Scientific Investigations Report 2005-5183, 35 p.

Phelps, H.L., 1993, Sediment toxicity of the Anacostia River estuary, Washington, DC: Bulletin of Environmental Contamination and Toxicology, v. 51, no. 4, p. 582-587.

Phelps, H.L., 2005, Identification of PCB, PAH and chlordane source areas in the Anacostia River watershed-Final report: Washington, D.C., prepared for the DC Water Resources Research Institute, May 2005, 14 p.

Radtke, D.B., 2005, Bottom-material samples (ver. 1.1): U.S. Geological Survey Techniques of Water-Resources Investigations, book 9, chap. A8, https://doi.org/10.3133/twri09A8.

Rasmussen, P.P., Gray, J.R., Glysson, D., and Ziegler, A.C., 2011, Guidelines and procedures for computing time-series suspended-sediment concentrations and loads from instream turbidity-sensor and streamflow data: U.S. Geological Survey Techniques and Methods, book 3, chap. C4, 52 p. (Also available at https://doi.org/10.3133/tm3C4.)

Rigaudy, J., and Klesney, S.P., eds., 1979, Nomenclature of organic chemistry-Sections A, B, C, D, E, F, and H: Oxford, U.K., Pergamon Press, 559 p.

Runkel, R.L., Crawford, C.G., and Cohn, T.A., 2004, Load Estimator (LOADEST) - A FORTRAN program for estimating constituent loads in streams and rivers: U.S. Geological Survey Techniques and Methods, book 4, chap. A5, 69 p., https://doi.org/10.3133/tm4A5.
Shreve, E.A., and Downs, A.C., 2005, Quality-assurance plan for the analysis of fluvial sediment by the U.S. Geological Survey Kentucky Water Science Center Sediment Laboratory: U.S. Geological Survey Open-File Report 2005-1230, $28 \mathrm{p}$.

Steinhauer, M.S., and Boehm, P.D., 1992, The compostion and distribution of saturated and aromatic hydrocarbons in nearshore sediment, river sediments, and coastal peat of the Alaskan Beaufort Sea-Implications for detecting anthropogenic hydrocarbon inputs: Marine Environmental Research, v. 33, no. 4, p. 223--253.

Sutherland, T.F., Lane, P.M., Amos, C.L., and Downing, J., 2000, The calibration of optical backscatter sensors for suspended sediment of varying darkness levels: Marine Geology, v. 162, no. 2-4, p. 587-597.

TetraTech, 2016, Phase I Remedial Investigation ReportAnacostia River Sediment Project, Washington, DC: Sterling, Virginia, TetraTech, prepared for Department of Energy \& Environment, Washington, D.C., March 18, 2016, 174 p., 13 app.

Turnipseed, D.P., and Sauer, V.B., 2010, Discharge measurements at gaging stations: U.S. Geological Survey Techniques and Methods, book 3, chap. A8, 87 p. (Also available at https://doi.org/10.3133/tm3A8.)

U.S. Army Corps of Engineers, 2009, Anacostia River Watershed Restoration Plan-Fort DuPont Subwatershed Provisional Restoration Project Inventory: Washington, D.C., Metropolitan Washington Council of Governments, July 2009, 90 p., accessed May 1, 2017, at https://doee.dc.gov/sites/default/files/dc/ sites/ddoe/publication/attachments/Appendix_E_ Fort-Dupont-Restoration-Inventory-July-2009.pdf.

U.S. Environmental Protection Agency, 2003, Amended decision rationale, Total Maximum Daily Loads, Anacostia River Watershed, for organics and metals: U.S. Environmental Protection Agency, October 2003, 31 p., accessed May 1, 2017, https://ddoe.dc.gov/sites/default/files/dc/sites/ ddoe/publication/attachments/amend_ana_om_1.pdf.

U.S. Geological Survey, 1991, Estimating loads from periodic records: U.S. Geological Survey Branch of Analysis Technical Memo 91.01, 81 p.

U.S. Geological Survey, 2000, Fort Dupont subwatershed restoration - 1999 baseline stream assessment study - Physical, chemical and biological conditions: Washington, D.C., Prepared for the Department of Environmental Programs, Metropolitan Washington Council of Governments, $70 \mathrm{p}$.

U.S. Geological Survey, 2019, USGS water data for the Nation: U.S. Geological Survey National Water Information System database, accessed December 16, 2017, at https:// doi.org/10.5066/F7P55KJN. 
U.S. Geological Survey, variously dated, National field manual for the collection of water-quality data: U.S. Geological Survey Techniques of Water-Resources Investigations, book 9, chaps. A1-A10, available at https://pubs.water.usgs. gov/twri9A.

Van den Berg, M., Birnbaum, L.S., Denison, M., De Vito, M., Farland, W., Feeley, M., Fiedler, H., Hakansson, H., Hanberg, A., Haws, L., Rose, M., Safe, S., Schrenk, D., Tohyama, C., Tritscher, A., Tuomisto, J., Tysklind, M., Walker, N., and Peterson, R.E., 2006, The 2005 World Health Organization reevaluation of human and Mammalian toxic equivalency factors for dioxins and dioxin-like compounds: Toxicological Sciences, v., 93, no. 2, p. 223-241.

Velinsky, D.J., Riedel, G.F., Ashley, J.T.F., and Cornwell, J.C., 2011, Historical contamination of the Anacostia River, Washington, D.C.: Environmental Monitoring Assessment, v. 183 , no. $1-4$, p. $307-328$.

Wagner, R.J., Boulger, R.W., Jr., Oblinger, C.J., and Smith, B.A., 2006 (ver. 1.0), Guidelines and standard procedures for continuous water-quality monitors-Station operation, record computation, and data reporting: U.S. Geological Survey Techniques and Methods 1-D3, 51 p., 8 attachments. (Also available at https://doi.org/10.3133/tm1D3.)

Warner, A., Shepp, D., Corish, K., and Galli, J., 1996, An existing source assessment of pollutants to the Anacostia Watershed: Washington, D.C., Metropolitan Washington Council of Governments, $230 \mathrm{p}$.

Washington, D.C., Department of Energy \& Environment, 2012, Anacostia River watershed implementation plan: District Department of the Environment, Watershed Protection Division, January 2012, 91 p., https://doee.dc.gov/sites/ default/files/dc/sites/ddoe/publication/attachments/Anacostia_WIP_2012_Final.pdf.

Wilson, T.P., 2019, Discharge and sediment data for selected tributaries to the Anacostia River, Washington, District of Columbia, 2003-18: U.S. Geological Survey data release, https://doi.org/10.5066/P9RUZSMV.

Wilson, T.P., and Bonin, J.L., 2007, Concentrations and loads od organic compounds and trace elements in tributaries to Newark and Raritan Bays, New Jersey: U.S. Geological Survey Scientific Investigations Report 2007-5059, 176 p.

Wilson, T.P., and Bonin, J.L., 2008, Occurrence of organic compounds and trace elements in the Upper Passaic and Elizabeth Rivers and their tributaries in New Jersey, July 2003 to February 2004_Phase II of the New Jersey Toxics Reduction Workplan for New York-New Jersey Harbor: U.S. Geological Survey Scientific Investigations Report 2007-5136, 42 p.
Wood, M.S., and Teasdale, G.N., 2013, Use of surrogate technologies to estimate suspended sediment in the Clearwater River, Idaho, and Snake River, Washington, 2008-10: U.S. Geological Survey Scientific Investigations Report 2013-5052, 30 p. 


\section{Appendix 1. Summary of stream discharge, precipitation, and sediment and contaminant loadings for the individual storms sampled in tributaries to the Anacostia River, 2017.}

This appendix presents metrics of the individual storms that were sampled on the gaged and ungaged tributaries to the Anacostia River. Concentrations of suspended sediment and particulate organic carbon and the continuous water-quality data and discharge data used in this report are available from the U.S. Geological Survey (USGS) National Water Information System (NWIS) database (U.S. Geological Survey, 2019), which can be accessed at https://waterdata. usgs.gov/md/nwis (see table 1 for USGS station identifiers). Data for precipitation at Ronald Reagan Washington National Airport were downloaded from MesoWest, accessed February 1, 2018, at https://mesowest.utah.edu/). 
Table 1.1. Time interval of data collection, total discharge, and sediment loads for the sampled storm events on tributaries to the Anacostia River.

[Mgal, million gallons; kg, kilograms]

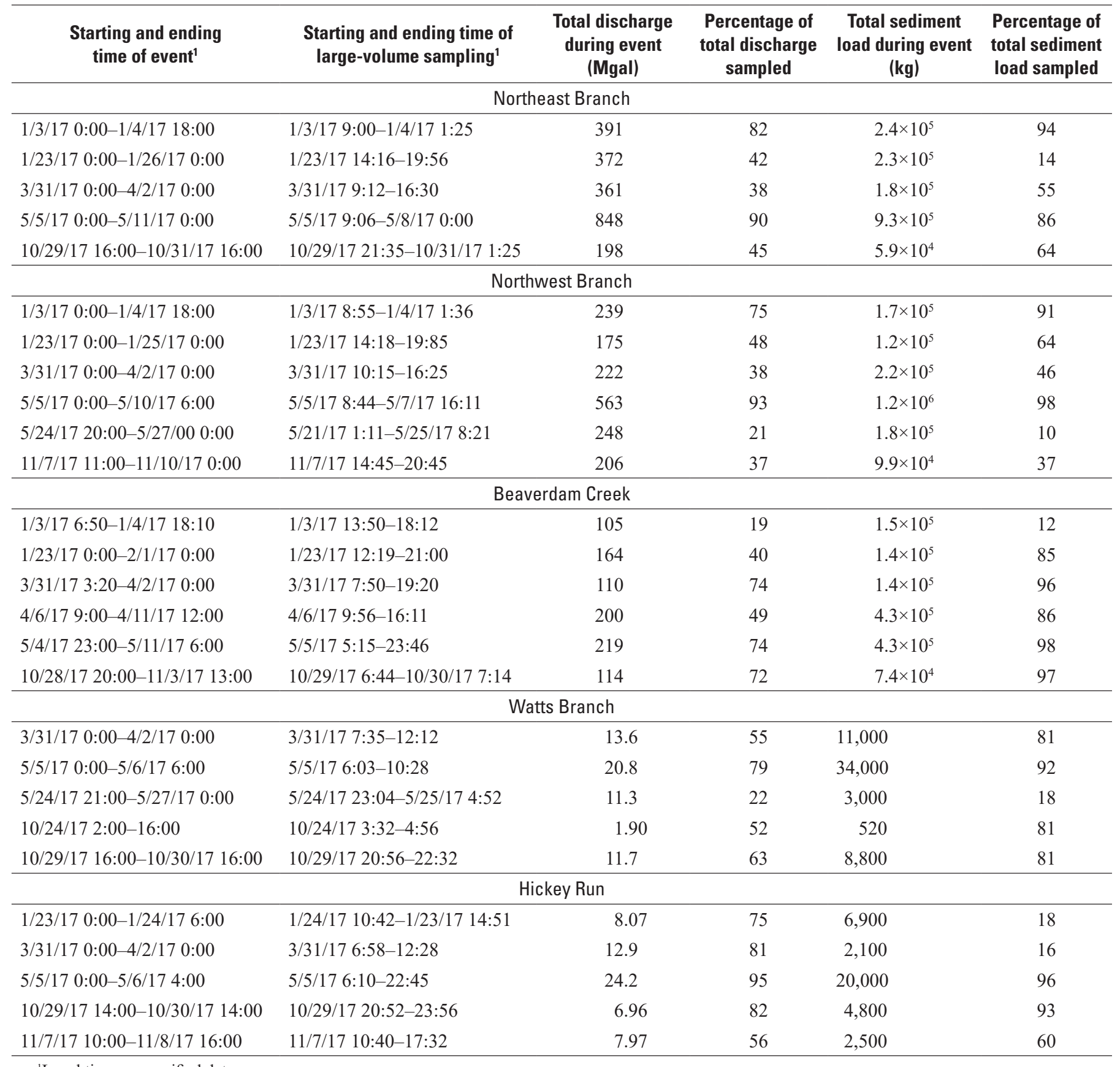

${ }^{1}$ Local time on specified date. 
Table 1.2. Summary of precipitation measured at Ronald Reagan Washington National Airport during sampled storms.

[<, less than; data from MesoWest, accessed February 1, 2018, at https://mesowest.utah.edu/]

\begin{tabular}{cccccc}
\hline $\begin{array}{c}\text { Sample } \\
\text { date }\end{array}$ & $\begin{array}{c}\text { Date/time } \\
\text { precipitation } \\
\text { started }\end{array}$ & $\begin{array}{c}\text { Date/time } \\
\text { precipitation } \\
\text { ended }\end{array}$ & $\begin{array}{c}\text { Total precipitation } \\
\text { for event, } \\
\text { in inches }\end{array}$ & $\begin{array}{c}\text { Duration } \\
\text { of event, } \\
\text { in hours }\end{array}$ & $\begin{array}{c}\text { Intensity, } \\
\text { in inches } \\
\text { per hour }\end{array}$ \\
\hline $1 / 3 / 2017$ & $1 / 2 / 20176: 35$ & $1 / 3 / 201717: 50$ & 1.213 & 44.2 & 0.027 \\
$1 / 22 / 2017$ & $1 / 22 / 20177: 52$ & $1 / 24 / 201711: 52$ & 0.871 & 52 & 0.017 \\
$3 / 31 / 2017$ & $3 / 31 / 20176: 47$ & $4 / 1 / 20178: 52$ & 0.95 & 26.08 & 0.036 \\
$4 / 6 / 2017$ & $4 / 6 / 20179: 21$ & $4 / 7 / 201720: 52$ & 1.57 & 35.52 & 0.044 \\
$5 / 5 / 2017$ & $5 / 5 / 20170: 33$ & $5 / 6 / 201722: 52$ & 1.831 & 46.32 & 0.039 \\
$5 / 25 / 2017$ & $5 / 25 / 20172: 52$ & $5 / 26 / 20170: 20$ & 0.792 & 21.47 & 0.039 \\
$7 / 17 / 2017$ & $7 / 17 / 201718: 41$ & $7 / 18 / 201718: 41$ & 0.001 & 24 & $<0.001$ \\
$7 / 28 / 2017$ & $7 / 28 / 20170: 52$ & $7 / 29 / 201821: 52$ & 3.801 & 45 & 0.085 \\
$8 / 7 / 2017$ & $8 / 7 / 20174: 52$ & $8 / 8 / 20179: 52$ & 1.521 & 29 & 0.052 \\
$8 / 12 / 2017$ & $8 / 11 / 201717: 52$ & $8 / 13 / 20172: 52$ & 1.32 & 33 & 0.039 \\
$8 / 29 / 2017$ & $8 / 29 / 20177: 40$ & $8 / 29 / 201723: 52$ & 0.981 & 16.2 & 0.061 \\
$10 / 9 / 2017$ & $10 / 9 / 20173: 30$ & $10 / 9 / 201715: 52$ & 0.611 & 12.37 & 0.049 \\
$10 / 24 / 2017$ & $10 / 24 / 20172: 52$ & $10 / 24 / 20179: 52$ & 0.135 & 7 & 0.019 \\
$10 / 29 / 2017$ & $10 / 29 / 20176: 52$ & $10 / 30 / 20179: 52$ & 1.065 & 27 & 0.039 \\
$11 / 7 / 2017$ & $11 / 7 / 20170: 00$ & $11 / 8 / 20175: 50$ & 1.076 & 29.87 & 0.036 \\
\hline
\end{tabular}


Table 1.3. Summary of discharge and sediment loads during sampled events on tributaries to the Anacostia River.

[L, liters; g, grams; ft, feet; Q, discharge; $\mathrm{ft}^{3} / \mathrm{s}$, cubic feet per second; Mgal/d, million gallons per day; g/d, grams per day; FNU, Formazin Nephelometric Units; SSC, suspended-sediment concentration; mg/L, milligrams per liter; $\mu \mathrm{g} / \mathrm{kg}$, micrograms per kilogram; kg, kilograms; kg/d, kilograms per day; mg/d, milligrams per day; na, not analyzed or not applicable; $\mathrm{PCB}$, polychlorinated biphenyls; PAH, polycyclic aromatic hydrocarbons; POC, particulate organic carbon; <, less than]

\begin{tabular}{|c|c|c|c|c|c|}
\hline Statistic (units) & $\begin{array}{c}\text { TS-NEB-LVSSD- } \\
\text { D-111616 }\end{array}$ & $\begin{array}{c}\text { TS-NEB-LVSS- } \\
\text { D-113017 }\end{array}$ & $\begin{array}{c}\text { TS-NWB-LVSSD- } \\
\text { D-111016 }\end{array}$ & $\begin{array}{c}\text { TS-NWB-LVSSD- } \\
\text { D-120717 }\end{array}$ & $\begin{array}{c}\text { TS-LBC-LVSSD- } \\
\text { D-102516 }\end{array}$ \\
\hline Stream & Northeast Branch & Northeast Branch & Northwest Branch & Northwest Branch & Beaverdam Creek \\
\hline Date & $11 / 16 / 2016$ & $11 / 30 / 2017$ & $11 / 10 / 2016$ & $12 / 7 / 2017$ & $10 / 25 / 2016$ \\
\hline Sample type & Low flow & Low flow & Low flow & Low flow & Low flow \\
\hline Blank & $\begin{array}{l}\text { TS-FB-LVSSD- } \\
\text { D-102516 }\end{array}$ & $\begin{array}{l}\text { TS-FB-LVSSD- } \\
\text { D-120717 }\end{array}$ & $\begin{array}{l}\text { TS-FB-LVSSD- } \\
\text { D-102516 }\end{array}$ & $\begin{array}{l}\text { TS-FB-LVSSD- } \\
\text { D-120717 }\end{array}$ & $\begin{array}{l}\text { TS-FB-LVSSD- } \\
\text { D-102516 }\end{array}$ \\
\hline Analyte group measured & PCB, PAH & PCB, PAH, pesticides & PCB, PAH & PCB, PAH, pesticides & PCB, PAH \\
\hline Volume filtered for PCB/PAH (L) & 1,005 & 623 & 1,000 & 620 & 1,000 \\
\hline Volume filtered for pesticides (L) & na & 611 & na & 694 & na \\
\hline Sediment mass for PCB and PAH (g) & 2.3 & 12.1 & 3.0 & 1.67 & 3.0 \\
\hline Sediment mass for pesticides $(\mathrm{g})$ & na & 11.9 & na & 1.87 & na \\
\hline Date and time event started & $11 / 16 / 168: 55$ & 11/30/17 9:20 & 11/10/16 9:00 & 12/7/17 9:30 & $10 / 25 / 1610: 30$ \\
\hline Gage height start (ft) & 2.7 & 0.82 & 1.35 & 1.33 & 0.10 \\
\hline Date and time event ended & $11 / 16 / 1614: 41$ & $11 / 30 / 1715: 30$ & $11 / 10 / 1615: 26$ & $12 / 7 / 1714: 42$ & $10 / 25 / 1614: 21$ \\
\hline Gage height end (ft) & 3.5 & 0.83 & 1.29 & 1.28 & 0.09 \\
\hline Average $\mathrm{Q}\left(\mathrm{ft}^{3} / \mathrm{s}\right)$ & 17.2 & 20 & 17.4 & 14.6 & 2.95 \\
\hline Average/maximum turbidity (FNU) & $3.0 / 9.0$ & $8.2 / 13$ & $197.3 / 8.0$ & $29 / 9$ & 39.2 \\
\hline Average/maximum SSC predicted (mg/L) & 0.49 & 8.8 & 10 & 11 & ${ }^{3} 17 / 40$ \\
\hline Total Q (Mgal/d) & 11.1 & 12.7 & 11.2 & 9.42 & 1.90 \\
\hline Total estimated sediment load (kg/d) & 141 & 263 & 413 & 401 & ${ }^{3} 70.8$ \\
\hline Total PCB concentration $(\mu \mathrm{g} / \mathrm{kg})$ & 19 & 0.99 & 20 & 7.4 & 310 \\
\hline Load of PCB $(g / d)$ & 0.027 & 0.0003 & 0.0083 & 0.0030 & 0.022 \\
\hline Total PAH concentration $(\mu \mathrm{g} / \mathrm{kg})$ & 2,700 & 240 & 7,700 & 3,900 & 2,300 \\
\hline Load of PAH $(g / d)$ & 0.38 & 0.063 & 3.2 & 1.6 & 0.16 \\
\hline
\end{tabular}


Table 1.3. Summary of discharge and sediment loads during sampled events on tributaries to the Anacostia River.-Continued

[L, liters; g, grams; ft, feet; Q, discharge; ft³/s, cubic feet per second; Mgal/d, million gallons per day; g/d, grams per day; FNU, Formazin Nephelometric Units; SSC, suspended-sediment concentration; mg/L, milligrams per liter; $\mu \mathrm{g} / \mathrm{kg}$, micrograms per kilogram; kg, kilograms; kg/d, kilograms per day; mg/d, milligrams per day; na, not analyzed or not applicable; PCB, polychlorinated biphenyls; PAH, polycyclic aromatic hydrocarbons; POC, particulate organic carbon; <, less than]

\begin{tabular}{|c|c|c|c|c|c|}
\hline Statistic (units) & $\begin{array}{c}\text { TS-LBC-LVSSD- } \\
\text { D-101817 }\end{array}$ & $\begin{array}{c}\text { TS-LBC-LVSSD-D- } \\
101817\end{array}$ & $\begin{array}{c}\text { TS-WB-LVSSD- } \\
\text { D-111617 }\end{array}$ & $\begin{array}{c}\text { TS-HR-LVSSD- } \\
\text { D-111716 }\end{array}$ & $\begin{array}{c}\text { TS-HR-LVSSD- } \\
\text { D-072717 }\end{array}$ \\
\hline Stream & Beaverdam Creek & Beaverdam Creek & Watts Branch & Hickey Run & Hickey Run \\
\hline Date & $10 / 18 / 2017$ & $10 / 19 / 2017$ & $11 / 16 / 2017$ & $11 / 17 / 2016$ & $7 / 27 / 2017$ \\
\hline Sample type & Low flow & Low flow & Low flow & Low flow & Low flow \\
\hline Blank & $\begin{array}{c}\text { TS-FB-LVSSD- } \\
\text { D-101817 }\end{array}$ & $\begin{array}{c}\text { TS-FB-LVSSD- } \\
\text { D-101817 }\end{array}$ & $\begin{array}{c}\text { TS-FB-LVSSD- } \\
\text { D-111617 }\end{array}$ & $\begin{array}{c}\text { TS-FB-LVSSD- } \\
\text { D-102516 }\end{array}$ & $\begin{array}{l}\text { TS-FB-LVSSD- } \\
\text { D-080217 }\end{array}$ \\
\hline Analyte group measured & PCB, РAH & Pesticides & PCB, PAH, pesticides & PCB, PAH & PCB, PAH, pesticides \\
\hline Volume filtered for PCB/PAH (L) & 931 & na & 575 & 1,000 & 467 \\
\hline Volume filtered for pesticides (L) & na & 710 & 507 & na & 294 \\
\hline Sediment mass for PCB and PAH $(\mathrm{g})$ & 3.9 & na & 2.3 & 5.5 & 2.8 \\
\hline Sediment mass for pesticides $(\mathrm{g})$ & na & 3.0 & 2.0 & na & 1.8 \\
\hline Date and time event started & $10 / 18 / 1710: 45$ & 10/19/17 9:42 & 11/16/17 9:20 & 11/17/16 9:22 & $7 / 27 / 1710: 20$ \\
\hline Gage height start (ft) & 0.1 & 0.09 & 4.21 & 1.77 & 1.71 \\
\hline Date and time event ended & $10 / 18 / 1712: 55$ & $10 / 19 / 1715: 24$ & $11 / 16 / 1714: 52$ & $11 / 17 / 1614: 47$ & $7 / 27 / 1717: 26$ \\
\hline Gage height end (ft) & 0.09 & 0.11 & 4.23 & 1.81 & 1.69 \\
\hline Average Q $\left(\mathrm{ft}^{3} / \mathrm{s}\right)$ & 7.28 & 6.61 & 1.25 & 0.47 & 0.26 \\
\hline Average/maximum turbidity (FNU) & $3.6 / 5.5$ & $4.2 / 10$ & $2.7 / 4.6$ & $4.1 / 11$ & $4.1 / 29.9$ \\
\hline Average/maximum SSC predicted $(\mathrm{mg} / \mathrm{L})$ & $4.6 / 8.7$ & $4.8 / 12$ & $3.6 / 7.3$ & $\mathrm{na} / 7.03$ & $6.0 / 21.9$ \\
\hline Total Q (Mgal/d) & 4.71 & 4.26 & 0.81 & 0.30 & 0.167 \\
\hline Total estimated sediment load (kg/d) & 96.7 & 92.3 & 6.98 & 7.91 & 2.24 \\
\hline Total PCB concentration $(\mu \mathrm{g} / \mathrm{kg})$ & 450 & na & 78 & 230 & 1,900 \\
\hline Load of PCB $(g / d)$ & 0.044 & na & 0.0005 & 0.0018 & 0.0043 \\
\hline Total PAH concentration $(\mu \mathrm{g} / \mathrm{kg})$ & 5,300 & na & 2,900 & 66,000 & 101,000 \\
\hline Load of PAH $(\mathrm{g} / \mathrm{d})$ & 2.4 & na & 0.020 & 0.52 & 0.10 \\
\hline
\end{tabular}


Table 1.3. Summary of discharge and sediment loads during sampled events on tributaries to the Anacostia River.—Continued

[L, liters; g, grams; ft, feet; Q, discharge; ft ${ }^{3} / \mathrm{s}$, cubic feet per second; Mgal/d, million gallons per day; g/d, grams per day; FNU, Formazin Nephelometric Units; SSC, suspended-sediment concentration; mg/L, milligrams per liter; $\mathrm{gg} / \mathrm{kg}$ micrograms per kilogram; $\mathrm{kg}$, kilograms; $\mathrm{kg} / \mathrm{d}$, kilograms per day; mg/d, milligrams per day; na, not analyzed or not applicable; $\mathrm{PCB}$, polychlorinated biphenyls; PAH, polycyclic aromatic hydrocarbons; POC, particulate organic carbon; $<$, less than]

\begin{tabular}{|c|c|c|c|}
\hline Statistic (units) & $\begin{array}{c}\text { TS-NR-LVSSD- } \\
\text { D-072517 }\end{array}$ & $\begin{array}{c}\text { TS-PB-LVSSD- } \\
\text { D-080217 } \\
\end{array}$ & $\begin{array}{c}\text { TS-FTS-LVSSD- } \\
\text { D-092017 }\end{array}$ \\
\hline Stream & Nash Run & Pope Branch & Fort Stanton Creek \\
\hline Date & $7 / 25 / 2017$ & $8 / 2 / 2017$ & $9 / 20 / 2017$ \\
\hline Sample type & Low flow & Low flow & Low flow \\
\hline Blank & $\begin{array}{c}\text { TS-EB-2-LVSSD- } \\
\text { W-072817 }\end{array}$ & $\begin{array}{c}\text { TS-EB-1-LVSSD- } \\
\text { W-080717 }\end{array}$ & $\begin{array}{l}\text { TS-FB-LVSSD- } \\
\text { D-092017 }\end{array}$ \\
\hline Analyte group measured & PCB, PAH, pesticides & PCB, PAH, pesticides & PCB, PAH, pesticides \\
\hline Volume filtered PCB and PAH (L) & 440 & 524 & 755 \\
\hline Volume filtered pesticides (L) & 490 & 414 & 612 \\
\hline Average SSC (mg/L) & 7.0 & 29 & 4.0 \\
\hline Average POC $(\mathrm{mg} / \mathrm{L})$ & $<0.27$ & na & $<0.49$ \\
\hline Sediment mass for PCB/PAH $(\mathrm{g})$ & 3.1 & 15.2 & 3.0 \\
\hline \multirow[t]{2}{*}{ Sediment mass for pesticides $(\mathrm{g})$} & 3.4 & 12.0 & 2.4 \\
\hline & \multicolumn{3}{|c|}{ Flow and loads in Watts Branch } \\
\hline Date and time event started & $7 / 25 / 170: 00$ & $8 / 2 / 170: 00$ & 9/20/17 0:00 \\
\hline Gage height start (ft) & 4.21 & 4.14 & 4.13 \\
\hline Date and time event ended & 7/26/17 0:00 & $8 / 2 / 1714: 50$ & 9/21/17 0:00 \\
\hline Gage height end (ft) & 4.13 & 4.12 & 4.12 \\
\hline Average $\mathrm{Q}\left(\mathrm{ft}^{3} / \mathrm{s}\right)$ & 1.06 & 1.16 & 1.11 \\
\hline Total Q (Mgal/d) & 0.69 & 0.75 & 0.72 \\
\hline Total sediment load $(\mathrm{kg} / \mathrm{d})$ & 6.21 & 6.33 & 3.65 \\
\hline \multirow[t]{2}{*}{ Basin area factor, normalized to Watts Branch } & 0.21 & 0.11 & 0.10 \\
\hline & \multicolumn{3}{|c|}{ Estimated loads in ungaged tributary } \\
\hline Total Q (Mgal/d) & 0.14 & 0.083 & 0.072 \\
\hline Sediment mass $(\mathrm{kg} / \mathrm{d})$ & 1.30 & 0.70 & 0.365 \\
\hline Total PCB concentration $(\mu \mathrm{g} / \mathrm{kg})$ & 36 & 1.1 & 7.3 \\
\hline Load of PCB (mg/d) & 0.046 & 0.0008 & 0.0027 \\
\hline Total PAH concentration $(\mu \mathrm{g} / \mathrm{kg})$ & 2,500 & 260 & 630 \\
\hline Load of PAH (mg/d) & 3.3 & 0.18 & 0.230 \\
\hline
\end{tabular}


Table 1.3. Summary of discharge and sediment loads during sampled events on tributaries to the Anacostia River.-Continued

[L, liters; g, grams; ft, feet; Q, discharge; ft³/s, cubic feet per second; Mgal/d, million gallons per day; g/d, grams per day; FNU, Formazin Nephelometric Units; SSC, suspended-sediment concentration; mg/L, milligrams per liter; $\mu \mathrm{g} / \mathrm{kg}$, micrograms per kilogram; kg, kilograms; kg/d, kilograms per day; mg/d, milligrams per day; na, not analyzed or not applicable; PCB, polychlorinated biphenyls; PAH, polycyclic aromatic hydrocarbons; POC, particulate organic carbon; $<$, less than]

\begin{tabular}{|c|c|c|c|c|c|}
\hline Statistic (units) & $\begin{array}{c}\text { TS-NEB-LVSSD- } \\
\text { W-010317 }\end{array}$ & $\begin{array}{c}\text { TS-NEB-LVSSD- } \\
\text { W-012317 }\end{array}$ & $\begin{array}{c}\text { TS-NEB-LVSSD- } \\
\text { W-033117 } \\
\end{array}$ & $\begin{array}{c}\text { TS-NEB-LVSSD- } \\
\text { W-050517 } \\
\end{array}$ & $\begin{array}{c}\text { TS-NEB-LVSSD- } \\
\text { W-102917 } \\
\end{array}$ \\
\hline Stream & Northeast Branch & Northeast Branch & Northeast Branch & Northeast Branch & Northeast Branch \\
\hline Date & $11 / 3 / 2017$ & $11 / 23 / 2017$ & $3 / 31 / 2017$ & $5 / 5 / 2017$ & $10 / 29 / 2017$ \\
\hline Sample type & Storm & Storm & Storm & Storm & Storm \\
\hline Blank & $\begin{array}{l}\text { TS-FB-LVSSD- } \\
\text { W-010317 }\end{array}$ & $\begin{array}{l}\text { TS-EB-LVSSD- } \\
\text { W-012317 }\end{array}$ & $\begin{array}{l}\text { TS-EB-LVSSD- } \\
\text { W-033117 }\end{array}$ & $\begin{array}{l}\text { TS-FB-LVSSD- } \\
\text { W-050517 }\end{array}$ & $\begin{array}{l}\text { TS-EB-LVSSD- } \\
\text { W-102917 }\end{array}$ \\
\hline Bed-sediment sample & na & $\begin{array}{c}\text { TS-NEB-SS- } \\
\text { W-012517 }\end{array}$ & na & $\begin{array}{c}\text { TS-NEB-SS- } \\
\text { W-052417 }\end{array}$ & $\begin{array}{c}\text { TS-NEB-SS- } \\
\text { W-110317 }\end{array}$ \\
\hline Analyte group measured & PCB, PAH & PCB, PAH & PCB, PAH & PCB, PAH, pesticides & PCB, PAH, pesticides \\
\hline Sediment mass for PCB and PAH $(\mathrm{g})$ & 60.9 & 40.5 & 55.1 & 48.5 & 55.7 \\
\hline Sediment mass for pesticides $(\mathrm{g})$ & na & na & na & 45.6 & 44.9 \\
\hline Date and time event started & $1 / 3 / 170: 00$ & 1/23/17 0:00 & $3 / 31 / 170: 00$ & $5 / 5 / 170: 00$ & 10/29/17 16:00 \\
\hline Gage height start (ft) & 1.16 & 0.90 & 0.82 & 0.80 & 0.84 \\
\hline Date and time event ended & 1/4/17 18:00 & 1/26/17 0:00 & 4/2/17 0:00 & 5/11/17 0:00 & 10/31/17 16:00 \\
\hline Gage height end (ft) & 1.16 & 0.98 & 1.12 & 0.84 & 0.84 \\
\hline Duration (minutes) & 2,525 & 4,325 & 2,885 & 8,645 & 2,885 \\
\hline Maximum $\mathrm{Q}\left(\mathrm{ft}^{3} / \mathrm{s}\right) /$ maximum gage height $(\mathrm{ft})$ & $1,020 / 3.32$ & $956 / 3.21$ & $874 / 3.06$ & $2,220 / 5.03$ & $761 / 2.85$ \\
\hline Date/time sampling started & 4/3/17 9:00 & $11 / 23 / 1714: 00$ & $3 / 31 / 179: 15$ & 5/5/17 8:06 & 10/29/17 21:17 \\
\hline Date/time sampling ended & $4 / 171: 25$ & $11 / 23 / 1720: 10$ & $3 / 31 / 1716: 30$ & $5 / 7 / 174: 21$ & $10 / 31 / 171: 27$ \\
\hline Total Q (Mgal) & 390 & 371.8 & 360.7 & 847.8 & 197.7 \\
\hline Percent of Q sampled & 77 & 34 & 38 & 81 & 84 \\
\hline Total sediment mass $(\mathrm{kg})$ & $2.77 \times 10^{5}$ & $2.58 \times 10^{5}$ & $1.75 \times 10^{5}$ & $1.20 \times 10^{6}$ & $5.88 \times 10^{4}$ \\
\hline Sediment load $(\mathrm{kg} / \mathrm{d})$ & $1.58 \times 10^{5}$ & $8.59 \times 10^{4}$ & $4.69 \times 10^{4}$ & $1.70 \times 10^{5}$ & $2.93 \times 10^{4}$ \\
\hline Percent of total sediment mass during sampling & 94 & 59 & 55 & 97 & 88 \\
\hline Average/maximum turbidity (FNU) & $65 / 198$ & $55 / 290$ & $50 / 150$ & $41 / 441$ & ${ }^{4} 33 / 93$ \\
\hline Average/maximum predicted SSC (mg/L) & $64 / 213$ & $86 / 325$ & $47 / 155$ & $41 / 520$ & ${ }^{4} 29 / 91$ \\
\hline Average measured SSC (mg/L) & 213 & 303 & 229 & 360 & na \\
\hline Average measured POC (mg/L) & 7.84 & 10.8 & 13.6 & 8.47 & 10.6 \\
\hline Total PCB concentration $(\mu \mathrm{g} / \mathrm{kg})$ & 2.8 & 6.2 & 1.4 & 6.4 & 4.3 \\
\hline Load of PCBs $(\mathrm{g})$ & 0.81 & 1.7 & 0.26 & 7.0 & 0.30 \\
\hline Load of PCBs $(\mathrm{g} / \mathrm{d})$ & 0.46 & 0.56 & 0.13 & 1.0 & 0.15 \\
\hline Total PAH concentration $(\mu \mathrm{g} / \mathrm{kg})$ & 1,500 & 3,200 & 1,110 & 1,800 & 3,200 \\
\hline Mass of PAH $(\mathrm{g})$ & 440 & 870 & 200 & 2,000 & 220 \\
\hline Load of PAH (g/d) & 250 & 290 & 100 & 290 & 110 \\
\hline
\end{tabular}


[L, liters; g, grams; ft, feet; Q, discharge; $\mathrm{ft}^{3} / \mathrm{s}$, cubic feet per second; Mgal/d, million gallons per day; g/d, grams per day; FNU, Formazin Nephelometric Units; SSC, suspended-sediment concentration; mg/L, milligrams per liter; $\mu \mathrm{g} / \mathrm{kg}$, micrograms per kilogram; $\mathrm{kg}$, kilograms; $\mathrm{kg} / \mathrm{d}$, kilograms per day; mg/d, milligrams per day; na, not analyzed or not applicable; $\mathrm{PCB}$, polychlorinated biphenyls; PAH, polycyclic aromatic hydrocarbons; POC, particulate organic carbon; <, less than]

\begin{tabular}{|c|c|c|c|c|c|}
\hline Statistic (units) & $\begin{array}{c}\text { TS-NWB-LVSSD- } \\
\text { W-010317 }\end{array}$ & $\begin{array}{c}\text { TS-NWB-LVSSD- } \\
\text { W-012317 }\end{array}$ & $\begin{array}{c}\text { TS-NWB-LVSSD- } \\
\text { W-033117 }\end{array}$ & $\begin{array}{c}\text { TS-NWB-LVSSD- } \\
\text { W-050517 }\end{array}$ & $\begin{array}{c}\text { TS-NWB-LVSSD- } \\
\text { W-052517 }\end{array}$ \\
\hline Stream & Northwest Branch & Northwest Branch & Northwest Branch & Northwest Branch & Northwest Branch \\
\hline Date & $1 / 3 / 2017$ & $1 / 23 / 2017$ & $3 / 31 / 2017$ & $5 / 5 / 2017$ & $5 / 25 / 2017$ \\
\hline Sample type & Storm & Storm & Storm & Storm & Storm \\
\hline Blank & $\begin{array}{l}\text { TS-FB-LVSSD- } \\
\text { W-010317 }\end{array}$ & $\begin{array}{l}\text { TS-NWB-SS- } \\
\text { W-012517 }\end{array}$ & $\begin{array}{l}\text { TS-EB-LVSSD- } \\
\text { W-033117 }\end{array}$ & $\begin{array}{l}\text { TS-FB-LVSSD- } \\
\text { W-050517 }\end{array}$ & $\begin{array}{l}\text { TS-EB-LVSSD- } \\
\text { W-052617 }\end{array}$ \\
\hline Bed-sediment sample & na & $\begin{array}{l}\text { TS-NWB-SS- } \\
\text { W-012517 }\end{array}$ & $\begin{array}{l}\text { TS-NWB-SS- } \\
\text { W-040117 }\end{array}$ & na & na \\
\hline Analyte group measured & PCB, PAH & PCB, PAH & PCB, PAH & PCB, PAH, pesticides & Pesticides \\
\hline Sediment mass for PCB and PAH (g) & 51.8 & 56.4 & 58.0 & 72.5 & na \\
\hline Sediment mass for pesticides $(\mathrm{g})$ & na & na & na & na & 32.6 \\
\hline Date and time event started & 1/3/17 0:00 & 1/23/17 0:00 & 3/31/17 0:00 & $5 / 5 / 170: 00$ & $5 / 24 / 1720: 00$ \\
\hline Gage height start (ft) & 1.62 & 1.4 & 1.29 & 1.29 & 1.61 \\
\hline Date and time event ended & 1/4/17 18:00 & 1/26/17 0:00 & 4/2/17 0:00 & 5/10/17 6:00 & 5/27/17 0:00 \\
\hline Gage height end (ft) & 1.66 & 1.4 & 1.57 & 1.29 & 1.62 \\
\hline Duration (minutes) & 2,525 & 4,325 & 2,885 & 7,565 & 3,125 \\
\hline Maximum $\mathrm{Q}\left(\mathrm{ft}^{3} / \mathrm{s}\right) /$ maximum gage height $(\mathrm{ft})$ & $760 / 3.31$ & $675 / 3.19$ & $635 / 3.13$ & $2,710 / 4.99$ & $571 / 3.03$ \\
\hline Date and time sampling started & $1 / 3 / 178: 55$ & 1/23/17 14:03 & $3 / 31 / 1710: 15$ & 5/5/17 9:41 & $5 / 24 / 171: 11$ \\
\hline Date and time sampling ended & $1 / 4 / 171: 36$ & $1 / 23 / 1720: 13$ & $3 / 31 / 1716: 25$ & 5/7/17 17:11 & $5 / 25 / 178: 21$ \\
\hline Total Q (Mgal) & 238.7 & 194.2 & 222 & 563 & 248 \\
\hline Percent of Q sampled & 78 & 36 & 38 & 71 & 16 \\
\hline Total sediment mass $(\mathrm{kg})$ & $1.68 \times 10^{5}$ & $1.25 \times 10^{5}$ & $2.20 \times 10^{5}$ & $1.19 \times 10^{6}$ & $1.78 \times 10^{5}$ \\
\hline Sediment load $(\mathrm{kg} / \mathrm{d})$ & $9.58 \times 10^{4}$ & $4.23 \times 10^{4}$ & $1.11 \times 10^{5}$ & $2.26 \times 10^{5}$ & $8.20 \times 10^{4}$ \\
\hline Percent of total sediment mass during sampling & 92 & 64 & 46 & 79 & 9.1 \\
\hline Average/maximum turbidity (FNU) & $61 / 277$ & $43 / 208$ & $84 / 331$ & $61 / 920$ & $82 / 329$ \\
\hline Average/maximum predicted SSC (mg/L) & $108 / 468$ & $68 / 410$ & $137 / 496$ & $110 / 1,593$ & $132 / 566$ \\
\hline Average measured SSC (mg/L) & 179 & 252 & 346 & 694 & 109 \\
\hline Average measured POC (mg/L) & 9.26 & 17.2 & 25.2 & 24.7 & 6.08 \\
\hline Total PCB concentration $(\mu \mathrm{g} / \mathrm{kg})$ & 1.3 & 3.3 & 3.1 & 1.8 & na \\
\hline Load of PCBs $(\mathrm{g})$ & 0.21 & 0.40 & 0.67 & 2.1 & na \\
\hline Load of PCBs $(g / d)$ & 0.12 & 0.13 & 0.33 & 0.40 & na \\
\hline Total PAH concentration $(\mu \mathrm{g} / \mathrm{kg})$ & 1,300 & 2,100 & 1,900 & 1,200 & na \\
\hline Mass of PAH (g) & 210 & 250 & 410 & 1,400 & na \\
\hline Load of PAH (g/d) & 120 & 84 & 210 & 270 & na \\
\hline
\end{tabular}


Table 1.3. Summary of discharge and sediment loads during sampled events on tributaries to the Anacostia River.-Continued

[L, liters; g, grams; ft, feet; Q, discharge; ft³/s, cubic feet per second; Mgal/d, million gallons per day; g/d, grams per day; FNU, Formazin Nephelometric Units; SSC, suspended-sediment concentration; mg/L, milligrams per liter; $\mu \mathrm{g} / \mathrm{kg}$, micrograms per kilogram; kg, kilograms; kg/d, kilograms per day; mg/d, milligrams per day; na, not analyzed or not applicable; PCB, polychlorinated biphenyls; PAH, polycyclic aromatic hydrocarbons; POC, particulate organic carbon; $<$, less than]

\begin{tabular}{|c|c|c|c|c|c|}
\hline Statistic (units) & $\begin{array}{c}\text { TS-NWB-LVSSD- } \\
\text { W-072817 }\end{array}$ & $\begin{array}{c}\text { TS-NWB-LVSSD- } \\
\text { W-110717 }\end{array}$ & $\begin{array}{c}\text { TS-LBC-LVSSD- } \\
\text { W-010317 }\end{array}$ & $\begin{array}{c}\text { TS-LBC-LVSSD- } \\
\text { W-012317 }\end{array}$ & $\begin{array}{c}\text { TS-LBC-LVSSD- } \\
\text { W-033117 }\end{array}$ \\
\hline Stream & Northwest Branch & Northwest Branch & Beaverdam Creek & Beaverdam Creek & Beaverdam Creek \\
\hline Date & $7 / 28 / 2017$ & $11 / 7 / 2017$ & $1 / 3 / 2017$ & $1 / 23 / 2017$ & $3 / 31 / 2017$ \\
\hline Sample type & Storm & Storm & Storm & Storm & Storm \\
\hline Blank & $\begin{array}{c}\text { TS-EB2-LVSSD- } \\
\text { W-072817 }\end{array}$ & $\begin{array}{l}\text { TS-EB-LVSSD- } \\
\text { W-110717 }\end{array}$ & $\begin{array}{l}\text { TS-FB-LVSSD- } \\
\text { W-010317 }\end{array}$ & $\begin{array}{l}\text { TS-EB-LVSSD- } \\
\text { W-012317 }\end{array}$ & $\begin{array}{l}\text { TS-EB-LVSSD- } \\
\text { W-033117 }\end{array}$ \\
\hline Bed-sediment sample & na & $\begin{array}{l}\text { TS-NWB-SS- } \\
\text { W-111517 }\end{array}$ & na & $\begin{array}{l}\text { TS-LBC-SS- } \\
\text { W-012517 }\end{array}$ & $\begin{array}{l}\text { TS-LBC-SS- } \\
\text { W-040117 }\end{array}$ \\
\hline Analyte group measured & Pesticides, metals & PCB, PAH, pesticides & PCB, PAH & $\mathrm{PCB}, \mathrm{PAH}$ & PCB, PAH \\
\hline Sediment mass for PCB and PAH $(\mathrm{g})$ & na & 29.9 & 230.2 & 64.1 & 53.6 \\
\hline Sediment mass for pesticides $(\mathrm{g})$ & 81.1 & 28.2 & na & na & na \\
\hline Date and time event started & $7 / 28 / 170: 00$ & 11/7/17 0:00 & $1 / 3 / 176: 50$ & 1/23/17 0:00 & $3 / 31 / 170: 00$ \\
\hline Gage height start (ft) & 1.17 & 1.35 & 0.44 & 0.39 & 0.19 \\
\hline Date and time event ended & 7/31/17 0:00 & 11/10/17 0:00 & 1/3/17 20:00 & $1 / 24 / 1713: 45$ & $4 / 2 / 170: 00$ \\
\hline Gage height end (ft) & 1.58 & 1.41 & 0.96 & 0.6 & 1.08 \\
\hline Duration (minutes) & 4,325 & 4,325 & 795 & 2,270 & 2,285 \\
\hline Maximum $\mathrm{Q}\left(\mathrm{ft}^{3} / \mathrm{s}\right) /$ maximum gage height $(\mathrm{ft})$ & $8,990 / 8.1$ & $614 / 3.22$ & $754 / 4.85$ & $\mathrm{na} / 4.99$ & $573 / 4.50$ \\
\hline Date and time sampling started & $7 / 28 / 1711: 20$ & 11/7/17 13:15 & $1 / 3 / 1713: 50$ & $1 / 23 / 1712: 19$ & $3 / 31 / 177: 50$ \\
\hline Date and time sampling ended & $7 / 28 / 1716: 15$ & $11 / 7 / 1720: 45$ & 1/3/17 18:15 & $1 / 23 / 1721: 00$ & 3/31/17 19:20 \\
\hline Total Q (Mgal) & 2,270 & 211 & 87.3 & 113 & 110 \\
\hline Percent of Q sampled & 23 & 35 & na & 58 & 74 \\
\hline Total sediment mass $(\mathrm{kg})$ & $8.93 \times 10^{6}$ & $1.01 \times 10^{5}$ & $1.5 \times 10^{5}$ & $1.3 \times 10^{5}$ & $1.4 \times 10^{5}$ \\
\hline Sediment load $(\mathrm{kg} / \mathrm{d})$ & $2.97 \times 10^{6}$ & $3.36 \times 10^{4}$ & $2.6 \times 10^{5}$ & $8.3 \times 10^{4}$ & $9.0 \times 10^{4}$ \\
\hline Percent of total sediment mass during sampling & 35 & 39 & na & 87 & 96 \\
\hline Average/maximum turbidity (FNU) & $180 / 985$ & $30 / 120$ & $180 / 466$ & $145 / 574$ & $170 / 453$ \\
\hline Average/maximum predicted SSC (mg/L) & $415 / 2,483$ & $52 / 250$ & $265 / 795$ & $142 / 647$ & $352 / 669$ \\
\hline Average measured SSC (mg/L) & 869 & na & 210 & 399 & 391 \\
\hline Average measured POC (mg/L) & 22.4 & na & 9.10 & 15.5 & 15.1 \\
\hline Total PCB concentration $(\mu \mathrm{g} / \mathrm{kg})$ & na & 1.2 & 5.4 & 26 & 36 \\
\hline Load of PCBs $(\mathrm{g})$ & na & 0.12 & 0.80 & 3.4 & 4.9 \\
\hline Load of PCBs $(g / d)$ & na & 0.039 & 1.4 & 2.2 & 7.2 \\
\hline Total PAH concentration $(\mu \mathrm{g} / \mathrm{kg})$ & na & 2,900 & 320 & 1,400 & 1,600 \\
\hline Mass of PAH $(\mathrm{g})$ & na & 280 & 47 & 180 & 220 \\
\hline Load of PAH $(\mathrm{g} / \mathrm{d})$ & na & 93 & 83 & 120 & 320 \\
\hline
\end{tabular}


[L, liters; g, grams; ft, feet; Q, discharge; $\mathrm{ft}^{3} / \mathrm{s}$, cubic feet per second; Mgal/d, million gallons per day; g/d, grams per day; FNU, Formazin Nephelometric Units; SSC, suspended-sediment concentration; mg/L, milligrams per liter; $\mu \mathrm{g} / \mathrm{kg}$, micrograms per kilogram; $\mathrm{kg}$, kilograms; kg/d, kilograms per day; mg/d, milligrams per day; na, not analyzed or not applicable; $\mathrm{PCB}$, polychlorinated biphenyls; PAH, polycyclic aromatic hydrocarbons; POC, particulate organic carbon; <, less than]

\begin{tabular}{|c|c|c|c|c|c|}
\hline Statistic (units) & $\begin{array}{c}\text { TS-LBC-LVSSD- } \\
\text { W-040617 }\end{array}$ & $\begin{array}{c}\text { TS-LBC-LVSSD- } \\
\text { W-050517 }\end{array}$ & $\begin{array}{c}\text { TS-LBC-LVSSD- } \\
\text { W-071817 }\end{array}$ & $\begin{array}{c}\text { TS-LBC-LVSSD- } \\
\text { W-072817 }\end{array}$ & $\begin{array}{c}\text { TS-LBC-LVSSD- } \\
\text { W-102917 }\end{array}$ \\
\hline Stream & Beaverdam Creek & Beaverdam Creek & Beaverdam Creek & Beaverdam Creek & Beaverdam Creek \\
\hline Date & $4 / 6 / 2017$ & $5 / 5 / 2017$ & $7 / 17 / 2017$ & $7 / 28 / 2017$ & $10 / 29 / 2017$ \\
\hline Sample type & Storm & Storm & Storm & Storm & Storm \\
\hline Blank & $\begin{array}{l}\text { TS-LBC-SS- } \\
\text { W-040717 }\end{array}$ & $\begin{array}{l}\text { TS-FB-LVSSD- } \\
\text { W-050517 }\end{array}$ & $\begin{array}{l}\text { TS-LBC-SS- } \\
\text { W-110317 }\end{array}$ & $\begin{array}{c}\text { TS-EB-2-LVSSD- } \\
\text { W-072817 }\end{array}$ & $\begin{array}{l}\text { TS-EB-LVSSD- } \\
\text { W-102917 }\end{array}$ \\
\hline Bed-sediment sample & $\begin{array}{c}\text { TS-LBC-SS- } \\
\text { W-040717 }\end{array}$ & $\begin{array}{c}\text { TS-LBC-SS- } \\
\text { W-052417 }\end{array}$ & na & $\begin{array}{c}\text { TS-EB1-SW- } \\
\text { W-071817 }\end{array}$ & $\begin{array}{l}\text { TS-LBC-SS- } \\
\text { W-110317 }\end{array}$ \\
\hline Analyte group measured & PCB, PAH & PCB, PAH, pesticides & Metals & Metals & PCB, PAH, pesticides \\
\hline Sediment mass for PCB and PAH (g) & 114.5 & 78.6 & na & na & 58.0 \\
\hline Sediment mass for pesticides $(\mathrm{g})$ & na & 56.6 & na & na & 35.2 \\
\hline Date and time event started & 4/6/17 9:00 & $5 / 4 / 1723: 00$ & 7/18/17 12:00 & $7 / 28 / 176: 00$ & 10/28/17 20:00 \\
\hline Gage height start (ft) & 0.79 & 0.20 & 0.14 & 0.16 & 0.08 \\
\hline Date and time event ended & 4/7/17 0:00 & $5 / 6 / 170: 30$ & 7/19/17 0:00 & 7/28/17 21:00 & 10/30/17 13:00 \\
\hline Gage height end (ft) & 1.47 & 1.1 & 0.68 & 2.6 & 0.57 \\
\hline Duration (minutes) & 905 & 1,535 & 725 & 905 & 2,465 \\
\hline Maximum $\mathrm{Q}\left(\mathrm{ft}^{3} / \mathrm{s}\right) /$ maximum gage height $(\mathrm{ft})$ & $1,090 / 6.37$ & $1,130 / 5.60$ & $670 / 3.54$ & $1,420 / 6.95$ & $568 / 3.43$ \\
\hline Date and time sampling started & 4/6/17 9:56 & $5 / 5 / 175: 15$ & $7 / 18 / 1716: 26$ & $7 / 28 / 1711: 35$ & $10 / 29 / 176: 44$ \\
\hline Date and time sampling ended & $4 / 6 / 1716: 11$ & $5 / 5 / 1723: 45$ & $7 / 18 / 1722: 20$ & $7 / 28 / 1717: 30$ & $10 / 30 / 1712: 14$ \\
\hline Total Q (Mgal) & 139 & 168 & 44.3 & 225 & 89.9 \\
\hline Percent of Q sampled & 70 & 97 & na & na & 91 \\
\hline Total sediment mass $(\mathrm{kg})$ & $4.1 \times 10^{5}$ & $4.2 \times 10^{5}$ & $7.5 \times 10^{4}$ & $6.1 \times 10^{5}$ & $7.2 \times 10^{4}$ \\
\hline Sediment load $(\mathrm{kg} / \mathrm{d})$ & $6.6 \times 10^{5}$ & $3.9 \times 10^{5}$ & $1.5 \times 10^{5}$ & $9.6 \times 10^{5}$ & $4.2 \times 10^{4}$ \\
\hline Percent of total sediment mass during sampling & 89 & 99 & na & na & 98 \\
\hline Average/maximum turbidity (FNU) & $390 / 1,110$ & $211 / 716$ & $165 / 515$ & $181 / 451$ & $51 / 568$ \\
\hline Average/maximum predicted SSC $(\mathrm{mg} / \mathrm{L})$ & $486 / 1,435$ & $284 / 1,065$ & $180 / 780$ & $402 / 1,053$ & $76 / 479$ \\
\hline Average measured SSC $(\mathrm{mg} / \mathrm{L})$ & 893 & 518 & 465 & 745 & 177 \\
\hline Average measured POC (mg/L) & 27.1 & 5.86 & 14.3 & 13.2 & 5.99 \\
\hline Total PCB concentration $(\mu \mathrm{g} / \mathrm{kg})$ & 14 & 140 & na & na & 52 \\
\hline Load of PCBs (g) & 5.8 & 59 & na & na & 3.7 \\
\hline Load of PCBs (g/d) & 9.2 & 55 & na & na & 2.2 \\
\hline Total PAH concentration $(\mu \mathrm{g} / \mathrm{kg})$ & 930 & 3,400 & na & na & 1,900 \\
\hline Mass of PAH $(\mathrm{g})$ & 390 & 1,400 & na & na & 140 \\
\hline Load of PAH (g/d) & 610 & 1,300 & na & na & 80 \\
\hline
\end{tabular}


Table 1.3. Summary of discharge and sediment loads during sampled events on tributaries to the Anacostia River.-Continued

[L, liters; g, grams; ft, feet; Q, discharge; ft³/s, cubic feet per second; Mgal/d, million gallons per day; g/d, grams per day; FNU, Formazin Nephelometric Units; SSC, suspended-sediment concentration; mg/L, milligrams per liter; $\mu \mathrm{g} / \mathrm{kg}$, micrograms per kilogram; kg, kilograms; kg/d, kilograms per day; mg/d, milligrams per day; na, not analyzed or not applicable; PCB, polychlorinated biphenyls; PAH, polycyclic aromatic hydrocarbons; POC, particulate organic carbon; $<$, less than]

\begin{tabular}{|c|c|c|c|c|c|}
\hline Statistic (units) & $\begin{array}{c}\text { TS-WB-LVSSD- } \\
\text { W-033117 }\end{array}$ & $\begin{array}{c}\text { TS-WB-LVSSD- } \\
\text { W-050517 }\end{array}$ & $\begin{array}{c}\text { TS-WB-LVSSD- } \\
\text { W-052417 }\end{array}$ & $\begin{array}{c}\text { TS-WB-LVSSD- } \\
\text { W-071817 }\end{array}$ & $\begin{array}{c}\text { TS-WB-LVSSD- } \\
\text { W-072217 }\end{array}$ \\
\hline Stream & Watts Branch & Watts Branch & Watts Branch & Watts Branch & Watts Branch \\
\hline Date & $3 / 31 / 2017$ & $5 / 5 / 2017$ & $5 / 24 / 2017$ & 7/17/2017 & $7 / 22 / 2017$ \\
\hline Sample type & Storm & Storm & Storm & Storm & Storm \\
\hline Blank & $\begin{array}{l}\text { TS-EB-LVSSD- } \\
\text { W-033117 }\end{array}$ & $\begin{array}{l}\text { TS-FB-LVSSD- } \\
\text { W-050517 }\end{array}$ & $\begin{array}{l}\text { TS-EB-LVSSD- } \\
\text { W-052617 }\end{array}$ & $\begin{array}{l}\text { TS-EB1-SW- } \\
\text { W-071817 }\end{array}$ & $\begin{array}{l}\text { TS-EB1-SW- } \\
\text { W-071817 }\end{array}$ \\
\hline Bed-sediment sample & $\begin{array}{c}\text { TS-WB-SS- } \\
\text { W-040117 } \\
\end{array}$ & na & $\begin{array}{c}\text { TS-WB-SS- } \\
\text { W-052417 }\end{array}$ & na & na \\
\hline Analyte group measured & PCB, PAH & PCB, PAH, pesticides & PCB, PAH, pesticides & Metals & Metals \\
\hline Sediment mass for PCB and PAH (g) & 36.9 & 45.7 & 31.6 & na & na \\
\hline Sediment mass for pesticides $(\mathrm{g})$ & na & 52.4 & 21.9 & na & na \\
\hline Date and time event started & $3 / 31 / 170: 00$ & $5 / 5 / 170: 00$ & $5 / 24 / 1721: 00$ & $7 / 18 / 1712: 00$ & $7 / 22 / 1713: 00$ \\
\hline Gage height start (ft) & 4.14 & 4.28 & 4.19 & 4.10 & 4.10 \\
\hline Date and time event ended & $4 / 2 / 170: 00$ & $5 / 6 / 176: 00$ & $5 / 27 / 170: 00$ & 7/19/17 6:00 & 7/23/17 8:00 \\
\hline Gage height end (ft) & 4.21 & 4.29 & 4.2 & 4.16 & 4.20 \\
\hline Duration (minutes) & 2,882 & 1,802 & 3,062 & 1,082 & 1,142 \\
\hline Maximum $\mathrm{Q}\left(\mathrm{ft}^{3} / \mathrm{s}\right) /$ maximum gage height $(\mathrm{ft})$ & $114 / 5.79$ & $350 / 6.72$ & $51.8 / 5.30$ & $283 / 6.52$ & $49.9 / 5.26$ \\
\hline Date and time sampling started & $3 / 31 / 177: 35$ & $5 / 5 / 176: 03$ & $5 / 24 / 1723: 01$ & $7 / 18 / 1716: 25$ & $7 / 22 / 1714: 09$ \\
\hline Date and time sampling ended & $3 / 31 / 1712: 12$ & 5/5/17 10:28 & $5 / 25 / 174: 52$ & 7/18/17 18:49 & 7/23/17 19:49 \\
\hline Total Q (Mgal) & 13.6 & 20.8 & 11.3 & 6.18 & 4.82 \\
\hline Percent of Q sampled & 55 & 65 & 20 & 72 & 66 \\
\hline Total sediment mass $(\mathrm{kg})$ & 11,000 & 34,000 & 3,000 & 12,000 & 1,500 \\
\hline Sediment load $(\mathrm{kg} / \mathrm{d})$ & 520 & 27,000 & 1,400 & 15,000 & 1,800 \\
\hline Percent of total sediment mass during sampling & 81 & 88 & 17 & 81 & 85 \\
\hline Average/maximum turbidity (FNU) & $39 / 194$ & $82 / 577$ & $25 / 153$ & $51 / 320$ & $30 / 138$ \\
\hline Average/maximum predicted SSC (mg/L) & $51 / 440$ & $118 / 1,002$ & $34 / 238$ & $61 / 940$ & $41 / 178$ \\
\hline Average measured SSC (mg/L) & 240 & 177 & 51 & 5.5 & na \\
\hline Average measured POC (mg/L) & 21.8 & na & 5.17 & 0.62 & na \\
\hline Total PCB concentration $(\mu \mathrm{g} / \mathrm{kg})$ & 5.1 & 20 & 4.8 & na & na \\
\hline Load of PCBs (g) & 0.055 & 0.69 & 0.0143 & na & na \\
\hline Load of PCBs $(\mathrm{g} / \mathrm{d})$ & 0.027 & 0.55 & 0.0068 & na & na \\
\hline Total PAH concentration $(\mu \mathrm{g} / \mathrm{kg})$ & 1,200 & 1,400 & 1,000 & na & na \\
\hline Mass of PAH $(\mathrm{g})$ & 13 & 48 & 3.0 & na & na \\
\hline Load of PAH (g/d) & 6.5 & 38 & 1.4 & na & na \\
\hline
\end{tabular}


[L, liters; g, grams; ft, feet; Q, discharge; $\mathrm{ft}^{3} / \mathrm{s}$, cubic feet per second; Mgal/d, million gallons per day; g/d, grams per day; FNU, Formazin Nephelometric Units; SSC, suspended-sediment concentration; mg/L, milligrams per liter; $\mu \mathrm{g} / \mathrm{kg}$, micrograms per kilogram; $\mathrm{kg}$, kilograms; $\mathrm{kg} / \mathrm{d}$, kilograms per day; mg/d, milligrams per day; na, not analyzed or not applicable; $\mathrm{PCB}$, polychlorinated biphenyls; $\mathrm{PAH}$, polycyclic aromatic hydrocarbons; POC, particulate organic carbon; <, less than]

\begin{tabular}{|c|c|c|c|c|c|}
\hline Statistic (units) & $\begin{array}{c}\text { TS-WB-LVSSD- } \\
\text { W-072317 }\end{array}$ & $\begin{array}{c}\text { TS-WB-LVSSD- } \\
\text { W-102417 }\end{array}$ & $\begin{array}{c}\text { TS-WB-LVSSD- } \\
\text { W-102917 }\end{array}$ & $\begin{array}{c}\text { TS-WB-LVSSD- } \\
\text { W-071817 }\end{array}$ & $\begin{array}{c}\text { TS-WB-LVSSD } \\
\text {-W-072217 }\end{array}$ \\
\hline Stream & Watts Branch & Watts Branch & Watts Branch & Watts Branch & Watts Branch \\
\hline Date & $7 / 23 / 2017$ & $10 / 24 / 2017$ & $10 / 29 / 2017$ & 7/17/2017 & $7 / 22 / 2017$ \\
\hline Sample type & Storm & Storm & Storm & Storm & Storm \\
\hline Blank & $\begin{array}{l}\text { TS-EB1-SW- } \\
\text { W-071817 }\end{array}$ & $\begin{array}{l}\text { TS-EB-LVSSD- } \\
\text { W-102917 }\end{array}$ & $\begin{array}{l}\text { TS-EB-LVSSD- } \\
\text { W-102917 }\end{array}$ & $\begin{array}{l}\text { TS-EB1-SW- } \\
\text { W-071817 }\end{array}$ & $\begin{array}{l}\text { TS-EB1-SW- } \\
\text { W-071817 }\end{array}$ \\
\hline Bed-sediment sample & na & $\begin{array}{l}\text { TS-WB-SS- } \\
\text { W-111317 }\end{array}$ & $\begin{array}{l}\text { TS-WB-SS- } \\
\text { W-111317 }\end{array}$ & na & na \\
\hline Analyte group measured & Metals & PCB, PAH, pesticides & PCB, PAH, pesticides & Metals & Metals \\
\hline Sediment mass for PCB and PAH $(\mathrm{g})$ & na & 21.4 & 45.6 & na & na \\
\hline Sediment mass for pesticides $(\mathrm{g})$ & na & 20.9 & 24.2 & na & na \\
\hline Date and time event started & $7 / 23 / 1713: 00$ & $10 / 24 / 172: 00$ & 10/29/17 16:00 & $7 / 18 / 1712: 00$ & $7 / 22 / 1713: 00$ \\
\hline Gage height start (ft) & 4.17 & 4.18 & 4.21 & 4.10 & 4.10 \\
\hline Date and time event ended & 7/23/17 21:00 & 10/24/17 16:00 & 10/30/17 16:00 & 7/19/17 6:00 & 7/23/17 8:00 \\
\hline Gage height end (ft) & 4.58 & 4.18 & 4.26 & 4.16 & 4.20 \\
\hline Duration (minutes) & 482 & 844 & 1,442 & 1,082 & 1,142 \\
\hline Maximum $\mathrm{Q}\left(\mathrm{ft}^{3} / \mathrm{s}\right) /$ maximum gage height $(\mathrm{ft})$ & $171 / 6.1$ & $26.6 / 4.88$ & $121 / 5.80$ & $283 / 6.52$ & $49.9 / 5.26$ \\
\hline Date and time sampling started & 7/23/17 14:09 & $10 / 24 / 173: 31$ & $10 / 29 / 1720: 55$ & $7 / 18 / 1716: 25$ & 7/22/17 14:09 \\
\hline Date and time sampling ended & $7 / 23 / 1720: 13$ & $10 / 24 / 174: 55$ & $10 / 29 / 1722: 31$ & 7/18/17 18:49 & 7/23/17 19:49 \\
\hline Total Q (Mgal) & 6.05 & 1.90 & 11.7 & 6.18 & 4.82 \\
\hline Percent of Q sampled & 81 & 40 & 28 & 72 & 66 \\
\hline Total sediment mass $(\mathrm{kg})$ & 9,700 & 480 & 8,800 & 12,000 & 1,500 \\
\hline Sediment load $(\mathrm{kg} / \mathrm{d})$ & 29,000 & 830 & 8,800 & 15,000 & 1,800 \\
\hline Percent of total sediment mass during sampling & 75 & 71 & 48 & 81 & 85 \\
\hline Average/maximum turbidity (FNU) & $336 / 999$ & $28 / 117$ & $46 / 232$ & $51 / 320$ & $30 / 138$ \\
\hline Average/maximum predicted SSC $(\mathrm{mg} / \mathrm{L})$ & $241 / 909$ & $28 / 136$ & $75 / 498$ & $61 / 940$ & $41 / 178$ \\
\hline Average measured SSC (mg/L) & na & 118 & 364 & 5.5 & na \\
\hline Average measured POC (mg/L) & na & na & 23 & 0.62 & na \\
\hline Total PCB concentration $(\mu \mathrm{g} / \mathrm{kg})$ & na & 23 & 76 & na & na \\
\hline Load of PCBs $(\mathrm{g})$ & na & 0.011 & 0.67 & na & na \\
\hline Load of PCBs $(\mathrm{g} / \mathrm{d})$ & na & 0.019 & 0.67 & na & na \\
\hline Total PAH concentration $(\mu \mathrm{g} / \mathrm{kg})$ & na & 1,800 & 4,100 & na & na \\
\hline Mass of PAH $(\mathrm{g})$ & na & 0.87 & 36 & na & na \\
\hline Load of PAH (g/d) & na & 1.5 & 36 & na & na \\
\hline
\end{tabular}


Table 1.3. Summary of discharge and sediment loads during sampled events on tributaries to the Anacostia River.-Continued

[L, liters; g, grams; ft, feet; Q, discharge; ft³/s, cubic feet per second; Mgal/d, million gallons per day; g/d, grams per day; FNU, Formazin Nephelometric Units; SSC, suspended-sediment concentration; mg/L, milligrams per liter; $\mu \mathrm{g} / \mathrm{kg}$, micrograms per kilogram; kg, kilograms; kg/d, kilograms per day; mg/d, milligrams per day; na, not analyzed or not applicable; PCB, polychlorinated biphenyls; PAH, polycyclic aromatic hydrocarbons; POC, particulate organic carbon; $<$, less than]

\begin{tabular}{|c|c|c|c|c|c|}
\hline Statistic (units) & $\begin{array}{c}\text { TS-WB-LVSSD- } \\
\text { W-072317 }\end{array}$ & $\begin{array}{c}\text { TS-WB-LVSSD- } \\
\text { W-102417 }\end{array}$ & $\begin{array}{c}\text { TS-WB-LVSSD- } \\
\text { W-102917 }\end{array}$ & $\begin{array}{c}\text { TS-HR-LVSSD- } \\
\text { W-012317 }\end{array}$ & $\begin{array}{l}\text { TS-HR-LVSSD- } \\
\text { W-033117 }\end{array}$ \\
\hline Stream & Watts Branch & Watts Branch & Watts Branch & Hickey Run & Hickey Run \\
\hline Date & 7/23/2017 & $10 / 24 / 2017$ & 10/29/2017 & $1 / 23 / 2017$ & $3 / 31 / 2017$ \\
\hline Sample type & Storm & Storm & Storm & Storm & Storm \\
\hline Blank & $\begin{array}{l}\text { TS-EB1-SW- } \\
\text { W-071817 }\end{array}$ & $\begin{array}{l}\text { TS-EB-LVSSD- } \\
\text { W-102917 }\end{array}$ & $\begin{array}{l}\text { TS-EB-LVSSD- } \\
\text { W-102917 }\end{array}$ & $\begin{array}{l}\text { TS-EB-LVSSD- } \\
\text { W-012317 }\end{array}$ & $\begin{array}{c}\text { TS-EB-LVSSD- } \\
\text { W-033117 }\end{array}$ \\
\hline Bed-sediment sample & na & $\begin{array}{l}\text { TS-WB-SS- } \\
\text { W-111317 }\end{array}$ & $\begin{array}{l}\text { TS-WB-SS- } \\
\text { W-111317 }\end{array}$ & $\begin{array}{l}\text { TS-HR-SS- } \\
\text { W-012517 }\end{array}$ & $\begin{array}{l}\text { TS-HR-SS- } \\
\text { W-040117 }\end{array}$ \\
\hline Analyte group measured & Metals & PCB, PAH, pesticides & PCB, PAH, pesticides & PCB, PAH & PCB, PAH \\
\hline Sediment mass for PCB and PAH (g) & na & 21.4 & 45.6 & 48.7 & 45.4 \\
\hline Sediment mass for pesticides $(\mathrm{g})$ & na & 20.9 & 24.2 & na & na \\
\hline Date and time event started & 7/23/17 13:00 & $10 / 24 / 172: 00$ & 10/29/17 16:00 & 1/23/17 0:00 & $3 / 31 / 170: 00$ \\
\hline Gage height start (ft) & 4.17 & 4.18 & 4.21 & 1.82 & 1.67 \\
\hline Date and time event ended & $7 / 23 / 1721: 00$ & 10/24/17 16:00 & 10/30/17 16:00 & $1 / 24 / 176: 00$ & $4 / 2 / 170: 00$ \\
\hline Gage height end (ft) & 4.58 & 4.18 & 4.26 & 2.00 & 1.78 \\
\hline Duration (minutes) & 482 & 844 & 1,442 & 1,802 & 2,882 \\
\hline Maximum Q ( $\left.\mathrm{ft}^{3} / \mathrm{s}\right) /$ maximum gage height $(\mathrm{ft})$ & $171 / 6.1$ & $26.6 / 4.88$ & $121 / 5.80$ & $179 / 4.64$ & $149 / 4.42$ \\
\hline Date and time sampling started & 7/23/17 14:09 & $10 / 24 / 173: 31$ & $10 / 29 / 1720: 55$ & $1 / 23 / 1710: 42$ & $3 / 31 / 176: 58$ \\
\hline Date and time sampling ended & $7 / 23 / 1720: 13$ & $10 / 24 / 174: 55$ & $10 / 29 / 1722: 31$ & $1 / 23 / 1714: 51$ & $3 / 31 / 1712: 26$ \\
\hline Total Q (Mgal) & 6.05 & 1.90 & 11.7 & 8.07 & 12.9 \\
\hline Percent of Q sampled & 81 & 40 & 28 & 64 & 81 \\
\hline Total sediment mass $(\mathrm{kg})$ & 9,700 & 480 & 8,800 & 6,800 & 2,100 \\
\hline Sediment load $(\mathrm{kg} / \mathrm{d})$ & 29,000 & 830 & 8,800 & 5,500 & 1,100 \\
\hline Percent of total sediment mass during sampling & 75 & 71 & 48 & 86 & 16 \\
\hline Average/maximum turbidity (FNU) & $336 / 999$ & $28 / 117$ & $46 / 232$ & $61 / 253$ & $41 / 169$ \\
\hline Average/maximum predicted SSC (mg/L) & $241 / 909$ & $28 / 136$ & $75 / 498$ & $83 / 504$ & $54 / 293$ \\
\hline Average measured SSC (mg/L) & na & 118 & 364 & 396 & 171 \\
\hline Average measured POC (mg/L) & na & na & 23 & 27.5 & 13.2 \\
\hline Total PCB concentration $(\mu \mathrm{g} / \mathrm{kg})$ & na & 23 & 76 & 42 & 20 \\
\hline Load of PCBs (g) & na & 0.011 & 0.67 & 0.29 & 0.15 \\
\hline Load of PCBs $(\mathrm{g} / \mathrm{d})$ & na & 0.019 & 0.67 & 0.23 & 0.074 \\
\hline Total PAH concentration $(\mu \mathrm{g} / \mathrm{kg})$ & na & 1,800 & 4,100 & 8,700 & 6,000 \\
\hline Mass of PAH $(\mathrm{g})$ & na & 0.87 & 36 & 59 & 44 \\
\hline Load of PAH (g/d) & na & 1.5 & 36 & 48 & 22 \\
\hline
\end{tabular}


[L, liters; g, grams; ft, feet; Q, discharge; $\mathrm{ft}^{3} / \mathrm{s}$, cubic feet per second; Mgal/d, million gallons per day; g/d, grams per day; FNU, Formazin Nephelometric Units; SSC, suspended-sediment concentration; mg/L, milligrams per liter; $\mu \mathrm{g} / \mathrm{kg}$, micrograms per kilogram; $\mathrm{kg}$, kilograms; $\mathrm{kg} / \mathrm{d}$, kilograms per day; mg/d, milligrams per day; na, not analyzed or not applicable; $\mathrm{PCB}$, polychlorinated biphenyls; $\mathrm{PAH}$, polycyclic aromatic hydrocarbons; POC, particulate organic carbon; <, less than]

\begin{tabular}{|c|c|c|c|c|}
\hline Statistic (units) & $\begin{array}{c}\text { TS-HR-LVSSD- } \\
\text { W-050517 }\end{array}$ & $\begin{array}{c}\text { TS-HR-LVSSD- } \\
\text { W-072817 }\end{array}$ & $\begin{array}{c}\text { TS-HR-LVSSD- } \\
\text { W-102917 }\end{array}$ & $\begin{array}{c}\text { TS-HR-LVSSD- } \\
\text { W-110717 }\end{array}$ \\
\hline Stream & Hickey Run & Hickey Run & Hickey Run & Hickey Run \\
\hline Date & $5 / 5 / 2017$ & $7 / 28 / 2017$ & $10 / 29 / 2017$ & $11 / 7 / 2017$ \\
\hline Sample type & Storm & Storm & Storm & Storm \\
\hline Blank & $\begin{array}{l}\text { TS-FB-LVSSD- } \\
\text { W-050517 }\end{array}$ & $\begin{array}{c}\text { TS-EB-2-LVSSD- } \\
\text { W-072817 }\end{array}$ & $\begin{array}{l}\text { TS-EB-LVSSD- } \\
\text { W-102917 }\end{array}$ & $\begin{array}{l}\text { TS-EB-LVSSD- } \\
\text { W-110717 }\end{array}$ \\
\hline Bed-sediment sample & $\begin{array}{l}\text { TS-HR-SS- } \\
\text { W-052417 }\end{array}$ & na & $\begin{array}{l}\text { TS-HR-SS- } \\
\text { W-111317 }\end{array}$ & $\begin{array}{l}\text { TS-HR-SS- } \\
\text { W-111517 }\end{array}$ \\
\hline Analyte group measured & PCB, PAH, pesticides & Metals & Pesticides & PCB, PAH, pesticides \\
\hline Sediment mass for PCB and PAH (g) & 57.4 & na & 20.7 & 42.2 \\
\hline Sediment mass for pesticides $(\mathrm{g})$ & 63.1 & na & 20.7 & 41.1 \\
\hline Date and time event started & $5 / 5 / 170: 00$ & 7/28/17 8:00 & $10 / 29 / 1714: 00$ & 11/7/17 10:00 \\
\hline Gage height start (ft) & 2.19 & 1.69 & 1.70 & 1.72 \\
\hline Date and time event ended & $5 / 6 / 174: 00$ & 7/30/17 0:00 & $10 / 30 / 1714: 00$ & 11/8/17 16:00 \\
\hline Gage height end (ft) & 2.14 & 2.0 & 1.83 & 1.84 \\
\hline Duration (minutes) & 1,682 & 2,402 & 1,442 & 1,802 \\
\hline Maximum $\mathrm{Q}\left(\mathrm{ft}^{3} / \mathrm{s}\right) /$ maximum gage height $(\mathrm{ft})$ & $976 / 7.82$ & 938/7.72 & $325 / 5.47$ & $52.8 / 3.49$ \\
\hline Date and time sampling started & $5 / 5 / 176: 11$ & 7/28/17 11:17 & 10/29/17 21:00 & $11 / 7 / 1711: 44$ \\
\hline Date and time sampling ended & $5 / 5 / 1722: 45$ & 7/29/17 4:00 & $10 / 29 / 1723: 24$ & $11 / 7 / 1717: 32$ \\
\hline Total Q (Mgal) & 24.2 & 59.0 & 6.96 & 7.97 \\
\hline Percent of Q sampled & 80 & 96 & 25 & 57 \\
\hline Total sediment mass $(\mathrm{kg})$ & 20,000 & 61,000 & 4,900 & 3,200 \\
\hline Sediment load $(\mathrm{kg} / \mathrm{d})$ & 18,000 & 537,000 & ${ }^{6} 4,900$ & 2,600 \\
\hline Percent of total sediment mass during sampling & 87 & 99 & 25 & 70 \\
\hline Average/maximum turbidity (FNU) & $110 / 348$ & $61 / 275$ & $34 / 193$ & $55 / 84$ \\
\hline Average/maximum predicted SSC (mg/L) & $120 / 766$ & $111 / 825$ & $57 / 490$ & $70 / 169$ \\
\hline Average measured SSC (mg/L) & 233 & 174 & na & 73 \\
\hline Average measured POC (mg/L) & 15.8 & ${ }^{5} 7.65$ & na & 12.5 \\
\hline Total PCB concentration $(\mu \mathrm{g} / \mathrm{kg})$ & 39 & na & na & 11 \\
\hline Load of PCBs $(\mathrm{g})$ & 0.80 & na & na & 0.035 \\
\hline Load of PCBs $(g / d)$ & 0.68 & na & na & 0.029 \\
\hline Total PAH concentration $(\mu \mathrm{g} / \mathrm{kg})$ & 4,500 & na & na & 6,000 \\
\hline Mass of PAH $(\mathrm{g})$ & 92 & na & na & 19 \\
\hline Load of PAH (g/d) & 79 & na & na & 16 \\
\hline
\end{tabular}


Table 1.3. Summary of discharge and sediment loads during sampled events on tributaries to the Anacostia River.-Continued

[L, liters; g, grams; ft, feet; Q, discharge; ft³/s, cubic feet per second; Mgal/d, million gallons per day; g/d, grams per day; FNU, Formazin Nephelometric Units; SSC, suspended-sediment concentration; mg/L, milligrams per liter; $\mu \mathrm{g} / \mathrm{kg}$, micrograms per kilogram; kg, kilograms; kg/d, kilograms per day; mg/d, milligrams per day; na, not analyzed or not applicable; PCB, polychlorinated biphenyls; PAH, polycyclic aromatic hydrocarbons; POC, particulate organic carbon; $<$, less than]

\begin{tabular}{|c|c|c|c|c|c|}
\hline Statistic (units) & $\begin{array}{l}\text { TS-NR-LVSSD- } \\
\text { W-072817 }\end{array}$ & $\begin{array}{l}\text { TS-PB-LVSSD- } \\
\text { W-080717 }\end{array}$ & $\begin{array}{l}\text { TS-FDP-LVSSD- } \\
\text { W-081217 }\end{array}$ & $\begin{array}{l}\text { TS-FDP-LVSSD- } \\
\text { W-082917 }\end{array}$ & $\begin{array}{l}\text { TS-FS-LVSSD- } \\
\text { W-100917 }\end{array}$ \\
\hline Stream & Nash Run & Pope Branch & Fort DuPont Creek & Fort DuPont Creek & Fort Stanton Creek \\
\hline Date & $7 / 28 / 2017$ & 8/7/2017 & $8 / 12 / 2017$ & $8 / 29 / 2017$ & $10 / 9 / 2017$ \\
\hline Sample type & Storm & Storm & Storm & Storm & Storm \\
\hline Blank & $\begin{array}{l}\text { TS-EB2-LVSSD- } \\
\text { W-072817 }\end{array}$ & $\begin{array}{l}\text { TS-EB1-LVSSD- } \\
\text { W-080717 }\end{array}$ & $\begin{array}{l}\text { TS-EB3-LVSSD- } \\
\text { W-081217 }\end{array}$ & $\begin{array}{l}\text { TS-EB-LVSSD- } \\
\text { W-082917 }\end{array}$ & $\begin{array}{l}\text { TS-EB-LVSSD- } \\
\text { W-110917 }\end{array}$ \\
\hline Bed-sediment sample & $\begin{array}{l}\text { TS-NR-SS- } \\
\text { W-080117 }\end{array}$ & $\begin{array}{l}\text { TS-PB-SS- } \\
\text { W-081417 }\end{array}$ & $\begin{array}{l}\text { TS-FDP-SS- } \\
\text { W-081417 }\end{array}$ & na & $\begin{array}{l}\text { TS-FS-SS- } \\
\text { W-101117 }\end{array}$ \\
\hline Analyte group measured & PCB, PAH, pesticides & PCB, PAH, pesticides & PCB, PAH, pesticides & PCB, PAH, pesticides & PCB, PAH, pesticides \\
\hline Sediment mass for PCB/PAH (g) & 41.7 & 23.6 & 46.5 & 67 & 32.6 \\
\hline Sediment mass for pesticides $(\mathrm{g})$ & 30.9 & 24.2 & 42.5 & 70 & 92.2 \\
\hline Date and time sampling started & $7 / 28 / 1711: 47$ & 8/7/17 12:28 & 8/12/17 19:12 & $9 / 2 / 176: 38$ & 10/9/17 6:16 \\
\hline Date and time sampling ended & 7/28/17 10:00 & 8/7/17 14:22 & $8 / 12 / 1720: 25$ & 9/2/17 9:29 & 10/9/17 8:11 \\
\hline Average measured SSC (mg/L) & 80 & 87 & 5,280 & 300 & 258 \\
\hline Average measured POC (mg/L) & 2.65 & 5.26 & 127 & na & na \\
\hline Total PCB concentration $(\mu \mathrm{g} / \mathrm{kg})$ & 47.7 & 0.925 & 0.496 & 1.88 & 1.32 \\
\hline \multirow{2}{*}{ Total PAH concentration $(\mu \mathrm{g} / \mathrm{kg})$} & 5,440 & 764 & 315 & 380 & 586 \\
\hline & \multicolumn{5}{|c|}{ Flow in Watts Branch } \\
\hline Date/Time event started & 7/28/17 10:00 & 8/7/17 0:00 & $8 / 12 / 1712: 00$ & 9/2/17 0:00 & 10/9/17 0:00 \\
\hline Gage height start (ft) & 4.14 & 4.11 & 4.24 & 4.14 & 4.14 \\
\hline Date/Time event ended & 7/30/17 0:00 & 8/9/17 0:00 & 8/14/17 0:00 & 9/5/17 0:00 & $10 / 10 / 176: 00$ \\
\hline Gage height end (ft) & 4.13 & 4.18 & 4.24 & 4.14 & 4.13 \\
\hline Duration (minutes) & 2,282 & 2,882 & 2,162 & 4,322 & 1,802 \\
\hline Maximum Q ( $\left.\mathrm{ft}^{3} / \mathrm{s}\right) /$ maximum gage height (ft) & $590 / 7.27$ & $192 / 6.19$ & $878 / 7.77$ & $69.2 / 5.36$ & $67.2 / 5.34$ \\
\hline Total Q (Mgal) & 61.3 & 15.3 & 27.2 & 10.3 & 4.98 \\
\hline \multirow[t]{2}{*}{ Total sediment mass $(\mathrm{kg})$} & $1.7 \times 10^{5}$ & 15,000 & $1.2 \times 10^{5}$ & 3,900 & 2,900 \\
\hline & \multicolumn{5}{|c|}{ Estimated in ungaged tributary } \\
\hline Basin area factor normalized to Watts Branch & 0.21 & 0.11 & 0.20 & 0.20 & 0.10 \\
\hline Total Q (Mgal) & 12.9 & 1.68 & 5.44 & 2.06 & 0.50 \\
\hline Total sediment mass $(\mathrm{kg})$ & 34,700 & 1,600 & 24,600 & 774 & 287 \\
\hline Total PCB concentration $(\mu \mathrm{g} / \mathrm{kg})$ & 48 & 0.92 & 0.50 & 1.9 & 1.3 \\
\hline Mass of PCBs $(\mathrm{g})$ & 1.7 & 0.0015 & 0.012 & 0.0015 & 0.0004 \\
\hline Total PAH concentration $(\mu \mathrm{g} / \mathrm{kg})$ & 5,500 & 760 & 320 & 380 & 590 \\
\hline Mass of PAH $(\mathrm{g})$ & 190 & 1.2 & 7.9 & 0.29 & 0.17 \\
\hline
\end{tabular}

${ }^{2}$ Missing turbidity measurements and suspended-sediment concentrations on 12/7/17.

${ }^{3}$ Turbidity sensor not yet installed; used suspended-sediment concentration of $8.8 \mathrm{mg} / \mathrm{L}$.

${ }^{4}$ Missing turbidity measurements 10/29 21:10-10/30 12:05.

${ }^{5}$ Missing 1,174 minutes of turbidity measurements.

${ }^{6}$ Missing 589 minutes of turbidity measurements, only through 10/30 5:22. 


\section{Reference Cited}

U.S. Geological Survey, 2019, USGS water data for the Nation: U.S. Geological Survey National Water Information System database, accessed December 16, 2017, at https://doi.org/10.5066/F7P55KJN. 


\section{Appendix 2. Summary of polychlorinated biphenyl, polycyclic aromatic hydrocarbon, pesticide, and metal concentrations in blank samples and suspended and bed sediment in tributaries to the Anacostia River, 2017.}

This appendix contains summaries of concentrations of polychlorinated biphenyls, polycyclic aromatic hydrocarbons, organochlorine pesticides, and trace metals in suspended sediment, bed sediment, and field and equipment blanks collected during the Anacostia River tributary study. All laboratory analytical results are available from the Washington, D.C., Department of Energy \& Environment by contacting:

Mr. Dev Murali

Washington, D.C., Department of Energy \& Environment

1200 First St NE, Washington, DC 20002

(202) 535-2600

dev.murali@dc.gov 
Table 2.1. Summary of total polychlorinated biphenyl, polycyclic aromatic hydrocarbon, and pesticide concentrations measured in field and equipment blanks.

[PCB, polychlorinated biphenyls; PAH, polycyclic aromatic hydrocarbons; $\mu \mathrm{g} / \mathrm{kg}$, micrograms per kilogram; nd, not detected]

\begin{tabular}{|c|c|c|c|c|c|c|c|c|c|}
\hline \multirow[b]{2}{*}{ Date } & \multirow[b]{2}{*}{ Sample identifier } & \multicolumn{4}{|c|}{ PCB } & \multicolumn{4}{|c|}{ PAH } \\
\hline & & $\begin{array}{c}\text { Number of } \\
\text { measurable } \\
\text { congeners }\end{array}$ & $\begin{array}{c}\text { Congener with } \\
\text { highest } \\
\text { concentration }\end{array}$ & $\begin{array}{c}\text { Maximum } \\
\text { concentration } \\
(\mu \mathrm{g} / \mathrm{kg})\end{array}$ & $\begin{array}{c}\text { Total } \\
\text { concentration } \\
(\mu \mathrm{g} / \mathrm{kg})\end{array}$ & $\begin{array}{l}\text { Number of } \\
\text { measurable } \\
\text { compounds }\end{array}$ & $\begin{array}{l}\text { Compound } \\
\text { with highest } \\
\text { concentration }\end{array}$ & $\begin{array}{c}\text { Maximum } \\
\text { concentration } \\
(\mu \mathrm{g} / \mathrm{kg})\end{array}$ & $\begin{array}{c}\text { Total } \\
\text { concentration } \\
(\mu \mathrm{g} / \mathrm{kg})\end{array}$ \\
\hline $11 / 16 / 16$ & TS-FB-LVSSD-D-102516 & 33 & PCB-11 & 0.021 & 0.11 & 1 & Chrysene & 0.48 & 0.48 \\
\hline $01 / 03 / 17$ & TS-EB-LVSSD-W-010317 & 27 & PCB-110/115 & 0.0086 & 0.10 & 0 & nd & nd & nd \\
\hline $01 / 23 / 17$ & TS-EB-LVSSD-W-012317 & 2 & PCB-90/101 & 0.0078 & 0.012 & 0 & nd & nd & nd \\
\hline $03 / 31 / 17$ & TS-EB-LVSSD-W-033117 & 45 & PCB-11 & 0.015 & 0.10 & 0 & nd & nd & nd \\
\hline $05 / 05 / 17$ & TS-FB-LVSSD-W-050517 & 14 & PCB-180/193 & 0.051 & 0.33 & 0 & nd & nd & nd \\
\hline $05 / 25 / 17$ & TS-EB-LVSSD-W-052617 & 32 & PCB-44/47/65 & 0.49 & 0.91 & 0 & nd & nd & nd \\
\hline $07 / 28 / 17$ & TS-EB-2-LVSSD-W-072817 & 34 & PCB-44/47/65 & 0.016 & 0.24 & 0 & nd & nd & nd \\
\hline 08/07/17 & TS-EB-1-LVSSD-W-080717 & 32 & PCB-180/193 & 0.034 & 0.30 & 0 & nd & nd & nd \\
\hline $08 / 12 / 17$ & TS-EB-1-LVSSD-W-081217 & 25 & PCB-61/70/74/76 & 0.01 & 0.13 & 0 & nd & nd & nd \\
\hline 08/29/17 & TS-EB-LVSSD-W-082917 & 14 & PCB-61/70/74/76 & 0.041 & 0.28 & 0 & nd & nd & nd \\
\hline 09/20/17 & TS-FB-LVSSD-D-092017 & 19 & PCB-52 & 0.10 & 0.93 & 3 & C3-Fluorenes & 25 & 48.3 \\
\hline $10 / 09 / 17$ & TS-EB-LVSSD-W-100917 & 26 & PCB-11 & 0.04 & 0.25 & 0 & nd & nd & nd \\
\hline $10 / 18 / 17$ & TS-FB-LVSSD-D-101817 & 43 & PCB-11 & 0.18 & 0.82 & 0 & nd & nd & nd \\
\hline $10 / 25 / 16$ & TS-FB-LVSSD-D-102516 & 33 & PCB-11 & 0.021 & 0.11 & 1 & Chrysene & 0.48 & 0.48 \\
\hline $10 / 29 / 17$ & TS-EB-LVSSD-102917 & 41 & PCB-11 & 0.038 & 0.28 & 0 & nd & nd & nd \\
\hline $11 / 07 / 17$ & TS-EB-LVSSD-W-110717 & 39 & PCB-11 & 0.053 & 0.27 & 0 & nd & nd & nd \\
\hline $11 / 16 / 17$ & TS-FB-LVSSD-D-111617 & 73 & PCB-11 & 0.12 & 1.2 & 2 & Fluorene & 12 & 12 \\
\hline $12 / 07 / 17$ & TS-FB-LVSSD-D-120717 & 39 & PCB-11 & 0.053 & 0.27 & 0 & nd & nd & nd \\
\hline
\end{tabular}


Table 2.1. Summary of total polychlorinated biphenyl, polycyclic aromatic hydrocarbon, and pesticide concentrations measured in field and equipment blanks.-Continued [PCB, polychlorinated biphenyls; PAH, polycyclic aromatic hydrocarbons; $\mu \mathrm{g} / \mathrm{kg}$, micrograms per kilogram; nd, not detected]

\begin{tabular}{|c|c|c|c|c|c|}
\hline \multirow[b]{2}{*}{ Date } & \multirow[b]{2}{*}{ Sample identifier } & \multicolumn{4}{|c|}{ Pesticide } \\
\hline & & $\begin{array}{c}\text { Number } \\
\text { pesticides } \\
\text { having } \\
\text { detectable } \\
\text { concentrations }\end{array}$ & $\begin{array}{l}\text { Congener with } \\
\text { highest } \\
\text { concentration }\end{array}$ & $\begin{array}{c}\text { Maximum } \\
\text { concentration } \\
(\mu \mathrm{g} / \mathrm{kg})\end{array}$ & $\begin{array}{c}\text { Total } \\
\text { concentration } \\
(\mu \mathrm{g} / \mathrm{kg})\end{array}$ \\
\hline $05 / 05 / 17$ & TS-FB-LVSSD-W-050517 & 0 & nd & nd & nd \\
\hline $05 / 25 / 17$ & TS-EB-LVSSD-W-052617 & 0 & nd & nd & nd \\
\hline $05 / 26 / 17$ & TS-EB-LVSSD-W-052617 & 0 & nd & nd & nd \\
\hline $07 / 28 / 17$ & TS-EB-2-LVSSD-W-072817 & 0 & nd & nd & nd \\
\hline $08 / 02 / 17$ & TS-EB-1-LVSSD-W-080717 & 0 & nd & nd & nd \\
\hline $08 / 12 / 17$ & TS-EB-1-LVSSD-W-081217 & 0 & nd & nd & nd \\
\hline $08 / 29 / 17$ & TS-EB-LVSSD-W-082917 & 0 & nd & nd & nd \\
\hline $09 / 20 / 17$ & TS-FB-LVSSD-D-092017 & 0 & nd & nd & nd \\
\hline $10 / 09 / 17$ & TS-EB-LVSSD-W-100917 & 0 & nd & nd & nd \\
\hline $10 / 18 / 17$ & TS-FB-LVSSD-D-101817 & 0 & nd & nd & nd \\
\hline $10 / 29 / 17$ & TS-EB-LVSSD-102917 & 1 & Heptachlor & 16 & 16 \\
\hline $11 / 07 / 17$ & TS-EB-LVSSD-W-110717 & 0 & nd & nd & nd \\
\hline $11 / 16 / 17$ & TS-FB-LVSSD-D-111617 & 0 & nd & nd & nd \\
\hline $12 / 07 / 17$ & TS-FB-LVSSD-D-120717 & 0 & nd & nd & nd \\
\hline
\end{tabular}


Table 2.2. Summary of polycyclic aromatic hydrocarbon concentrations in suspended-sediment samples from tributaries to the Anacostia River.

$[\mu \mathrm{g} / \mathrm{kg}$, micrograms per kilogram; nd, not detected]

\begin{tabular}{|c|c|c|c|c|c|c|c|c|}
\hline & \multirow{2}{*}{$\begin{array}{l}\text { Number } \\
\text { detected }\end{array}$} & \multicolumn{3}{|c|}{ Concentration ( $\mu \mathrm{g} / \mathrm{kg}$ ) } & \multirow{2}{*}{$\begin{array}{l}\text { Number } \\
\text { detected }\end{array}$} & \multicolumn{3}{|c|}{ Concentration $(\mu \mathrm{g} / \mathrm{kg})$} \\
\hline & & Average & Maximum & Minimum & & Average & Maximum & Minimum \\
\hline & \multicolumn{4}{|c|}{ Northeast Branch } & \multicolumn{4}{|c|}{ Northwest Branch } \\
\hline Acenaphthene & 7 & 4.7 & 8.0 & 1.2 & 7 & 6.2 & 9.0 & 4.1 \\
\hline Acenapthylene & 6 & 3.0 & 4.4 & 1.8 & 6 & 4.5 & 12. & 1.4 \\
\hline Anthracene & 7 & 14 & 43 & 1.7 & 7 & 17 & 31 & 8.3 \\
\hline Benzo(a)anthracene & 7 & 76 & 140 & 8.2 & 7 & 110 & 230 & 52 \\
\hline Benzo(a)pyrene & 7 & 95 & 160 & 9.7 & 7 & 140 & 370 & 57 \\
\hline Benzo(b)fluoranthene & 7 & 198 & 330 & 21 & 7 & 290 & 830 & 110 \\
\hline Benzo(e)pyrene & 7 & 116 & 190 & 13 & 7 & 170 & 500 & 58 \\
\hline Benzo(g,h,i)perylene & 7 & 116 & 210 & 13 & 7 & 170 & 500 & 56 \\
\hline Benzo(k)fluoranthene & 7 & 82 & 150 & 7.7 & 7 & 120 & 370 & 47 \\
\hline Chrysene & 7 & 170 & 310 & 20 & 7 & 270 & 730 & 99 \\
\hline C1-Chrysenes/Benzo(a)anthracenes & 7 & 74 & 130 & 8.7 & 7 & 120 & 310 & 44 \\
\hline C2-Chrysenes/Benzo(a)anthracenes & 7 & 43 & 79. & 4.6 & 7 & 74 & 220 & 22 \\
\hline C3-Chrysenes/Benzo(a)anthracenes & 7 & 26 & 51 & 2.9 & 7 & 47 & 140 & 12 \\
\hline C4-Chrysenes/Benzo(a)anthracenes & 7 & 14 & 31 & 1.7 & 7 & 24 & 67 & 6.6 \\
\hline Dibenzo(a,h)anthracene & 7 & 20 & 33 & 2.1 & 7 & 31 & 97 & 11 \\
\hline Fluoranthene & 7 & 240 & 460 & 27 & 7 & 320 & 770 & 160 \\
\hline C1-Fluoranthenes/pyrenes & 6 & 80 & 120 & 40 & 6 & 110 & 250 & 46 \\
\hline Fluorene & 6 & 6.9 & 9.6 & 4.4 & 6 & 8.6 & 16 & 4.6 \\
\hline C1-Fluorene & 5 & 4.3 & 7.8 & 2.3 & 5 & 8.4 & 15 & 2.6 \\
\hline C2-Fluorene & 6 & 8.0 & 16 & 2.9 & 6 & 16 & 36 & 3.3 \\
\hline C3-Fluorene & 7 & 11 & 34 & 3.3 & 6 & 22 & 50 & 3.7 \\
\hline Indeno(1,2,3-cd)pyrene & 7 & 85 & 140. & 9.2 & 7 & 120 & 370 & 45 \\
\hline Naphthalene & 2 & 14 & 16 & 11 & 5 & 14 & 19 & 6.5 \\
\hline 1-Methylnaphthalene & 4 & 3.9 & 6.1 & 2.3 & 5 & 12 & 40 & 2.8 \\
\hline 2-Methylnaphthalene & 2 & 6.5 & 8.0 & 5.0 & 5 & 7.7 & 13 & 3.3 \\
\hline C2-Naphthalenes & 4 & 5.3 & 8.1 & 4.0 & 4 & 6.8 & 8.6 & 3.8 \\
\hline C3-Naphthalenes & 4 & 5.2 & 7.8 & 3.1 & 5 & 12 & 31 & 4.0 \\
\hline C4-Naphthalenes & 7 & 7.3 & 16 & 3.6 & 6 & 18 & 40 & 3.2 \\
\hline Perylene & 7 & 32 & 52 & 6.7 & 7 & 42 & 110 & 17 \\
\hline Phenanthrene & 7 & 110 & 190 & 11 & 7 & 150 & 290 & 80 \\
\hline C1-Phenanthrenes/anthracenes & 7 & 26 & 43 & 13 & 7 & 59 & 150 & 18 \\
\hline C2-Phenanthrenes/anthracenes & 6 & 43 & 100 & 16 & 7 & 110 & 440 & 11 \\
\hline C3-Phenanthrenes/anthracenes & 7 & 54 & 190 & 12 & 7 & 150 & 370 & 4.2 \\
\hline C4-Phenanthrenes/anthracenes & 6 & 24 & 55 & 7.6 & 6 & 62 & 140 & 10 \\
\hline Pyrene & 7 & 180 & 380 & 11. & 7 & 200 & 600 & 11 \\
\hline
\end{tabular}


Table 2.2. Summary of polycyclic aromatic hydrocarbon concentrations in suspended-sediment samples from tributaries to the Anacostia River-Continued

$[\mu \mathrm{g} / \mathrm{kg}$, micrograms per kilogram; nd, not detected]

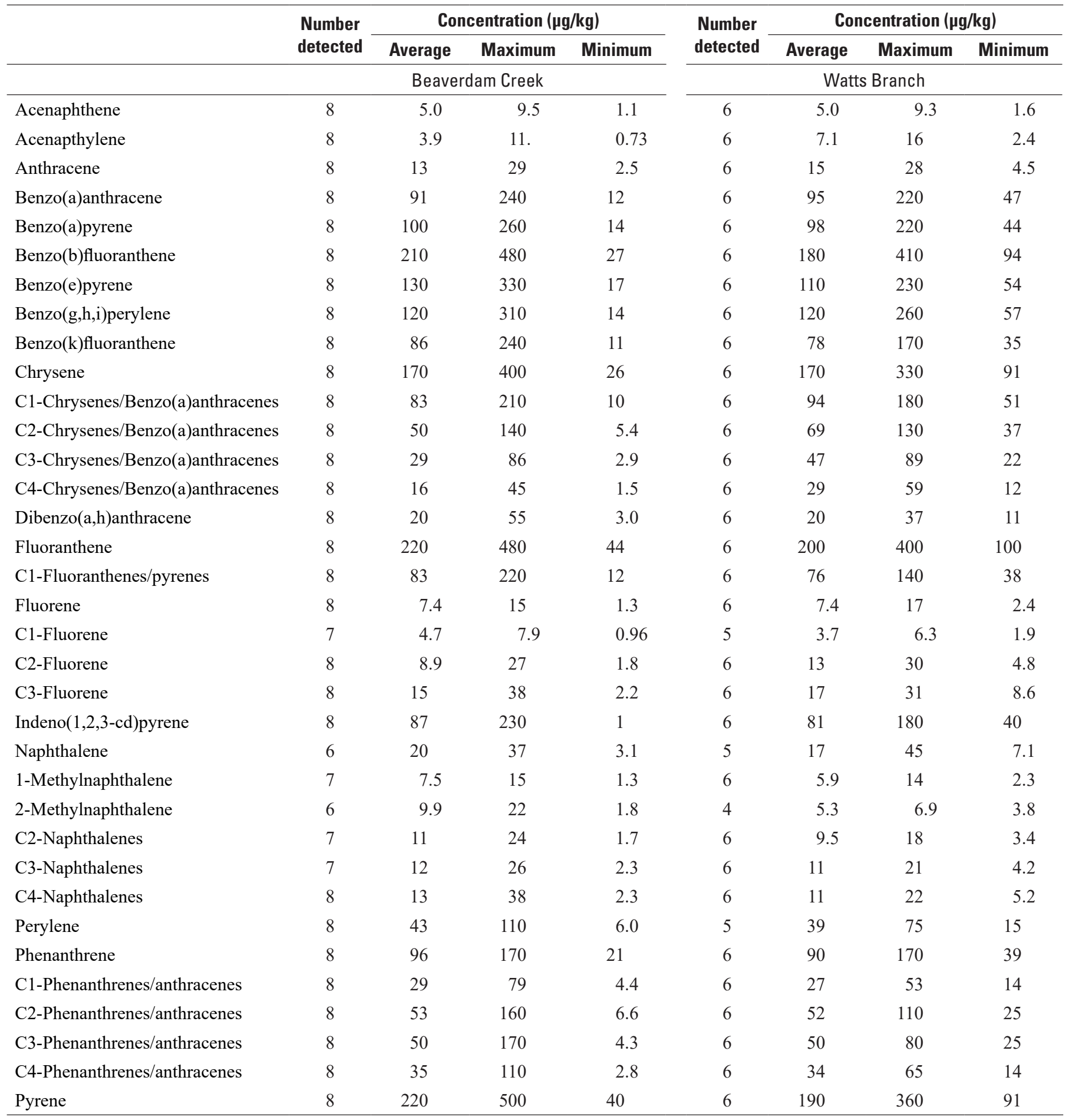


Table 2.2. Summary of polycyclic aromatic hydrocarbon concentrations in suspended-sediment samples from tributaries to the Anacostia River.-Continued

$[\mu \mathrm{g} / \mathrm{kg}$, micrograms per kilogram; nd, not detected]

\begin{tabular}{|c|c|c|c|c|c|c|c|c|}
\hline & \multirow{2}{*}{$\begin{array}{l}\text { Number } \\
\text { detected }\end{array}$} & \multicolumn{3}{|c|}{ Concentration $(\mu \mathrm{g} / \mathrm{kg})$} & \multirow{2}{*}{$\begin{array}{l}\text { Number } \\
\text { detected }\end{array}$} & \multicolumn{3}{|c|}{ Concentration $(\mu \mathrm{g} / \mathrm{kg})$} \\
\hline & & Average & Maximum & Minimum & & Average & Maximum & Minimum \\
\hline & \multicolumn{4}{|c|}{ Hickey Run } & \multicolumn{4}{|c|}{ Nash Run } \\
\hline Acenaphthene & 5 & 23 & 44 & 14 & 2 & 18 & 28. & 8.9 \\
\hline Acenapthylene & 5 & 17 & 42 & 6.3 & 2 & 7.1 & 7.9 & 6.4 \\
\hline Anthracene & 5 & 40 & 66 & 27 & 2 & 32 & 48 & 16 \\
\hline Benzo(a)anthracene & 5 & 290 & 490 & 190 & 2 & 220 & 330 & 100 \\
\hline Benzo(a)pyrene & 5 & 280 & 420 & 190 & 2 & 210 & 320 & 110 \\
\hline Benzo(b)fluoranthene & 5 & 590 & 1,000 & 370 & 2 & 350 & 480 & 210 \\
\hline Benzo(e)pyrene & 5 & 370 & 710 & 220 & 2 & 210 & 280 & 130 \\
\hline Benzo(g,h,i)perylene & 5 & 330 & 600 & 220 & 2 & 220 & 310 & 130 \\
\hline Benzo(k)fluoranthene & 5 & 230 & 380 & 150 & 2 & 180 & 260 & 99 \\
\hline Chrysene & 5 & 550 & 1,000 & 340 & 2 & 330 & 450 & 210 \\
\hline C1-Chrysenes/Benzo(a)anthracenes & 5 & 440 & 1,100 & 210 & 2 & 170 & 230 & 110 \\
\hline C2-Chrysenes/Benzo(a)anthracenes & 5 & 320 & 760 & 140 & 2 & 110 & 150 & 79 \\
\hline C3-Chrysenes/Benzo(a)anthracenes & 5 & 200 & 490 & 89 & 2 & 72 & 93 & 50 \\
\hline C4-Chrysenes/Benzo(a)anthracenes & 5 & 110 & 270 & 46 & 2 & 4 & 54 & 26 \\
\hline Dibenzo(a,h)anthracene & 5 & 52 & 96 & 35 & 2 & 40 & 58 & 23 \\
\hline Fluoranthene & 5 & 550 & 980 & 340 & 2 & 410 & 590 & 230 \\
\hline C1-Fluoranthenes/pyrenes & 5 & 1,400 & 6,000 & 180 & 2 & 140 & 170 & 110 \\
\hline Fluorene & 5 & 58 & 170 & 20 & 2 & 14 & 22 & 6.6 \\
\hline C1-Fluorene & 5 & 180 & 730 & 17 & 1 & 8.0 & 8.0 & 8.0 \\
\hline C2-Fluorene & 5 & 770 & 3,500 & 30 & 2 & 16 & 16 & 16 \\
\hline C3-Fluorene & 5 & 1,400 & 6,200 & 49 & 2 & 30 & 32 & 29 \\
\hline Indeno(1,2,3-cd)pyrene & 5 & 230 & 400 & 160 & 2 & 160 & 230 & 96 \\
\hline Naphthalene & 4 & 38 & 6.0 & 23 & nd & nd & nd & nd \\
\hline 1-Methylnaphthalene & 5 & 39 & 110 & 17 & 1 & 13 & 13 & 13 \\
\hline 2-Methylnaphthalene & 5 & 39 & 96 & 19 & 1 & 18 & 18 & 18 \\
\hline C2-Naphthalenes & 5 & 160 & 560 & 41 & 2 & 25 & 27 & 22 \\
\hline C3-Naphthalenes & 5 & 930 & 4,200 & 55 & 2 & 22 & 22 & 21 \\
\hline C4-Naphthalenes & 5 & 1,750 & 8,000 & 61 & 2 & 24 & 26 & 23 \\
\hline Perylene & 5 & 91 & 170 & 61 & 2 & 84 & 110 & 57 \\
\hline Phenanthrene & 5 & 440 & 820 & 220 & 2 & 190 & 300 & 80 \\
\hline C1-Phenanthrenes/anthracenes & 5 & 570 & 2,400 & 66 & 2 & 44 & 56 & 33 \\
\hline C2-Phenanthrenes/anthracenes & 5 & 1,900 & 8,500 & 120 & 2 & 68 & 78 & 57 \\
\hline C3-Phenanthrenes/anthracenes & 5 & 1,800 & 7,800 & 110 & 2 & 95 & 110 & 82 \\
\hline C4-Phenanthrenes/anthracenes & 5 & 1,000 & 4,500 & 70 & 2 & 43 & 44 & 41 \\
\hline Pyrene & 5 & 1,100 & 3,600 & 370 & 2 & 360 & 510 & 214 \\
\hline
\end{tabular}


Table 2.2. Summary of polycyclic aromatic hydrocarbon concentrations in suspended-sediment samples from tributaries to the Anacostia River.-Continued

$[\mu \mathrm{g} / \mathrm{kg}$, micrograms per kilogram; nd, not detected]

\begin{tabular}{|c|c|c|c|c|}
\hline & \multirow{2}{*}{$\begin{array}{l}\text { Number } \\
\text { detected }\end{array}$} & \multicolumn{3}{|c|}{ Concentration $(\mu \mathrm{g} / \mathrm{kg})$} \\
\hline & & Average & Maximum & Minimum \\
\hline & \multicolumn{4}{|c|}{ Pope Branch } \\
\hline Acenapthylene & 2 & 2.8 & 3.0 & 2.6 \\
\hline Anthracene & 2 & 3.3 & 5.5 & 1.1 \\
\hline Benzo(a)pyrene & 2 & 19 & 35 & 3.5 \\
\hline Benzo(b)fluoranthene & 2 & 32 & 56 & 7.6 \\
\hline Benzo(e)pyrene & 2 & 20 & 35 & 4.1 \\
\hline Benzo(g,h,i)perylene & 2 & 23 & 42 & 4.5 \\
\hline Benzo(k)fluoranthene & 2 & 15 & 28 & 2.6 \\
\hline C3-Chrysenes/Benzo(a)anthracenes & 2 & 13 & 19 & 6.6 \\
\hline C4-Chrysenes/Benzo(a)anthracenes & 2 & 9.3 & 12 & 6.6 \\
\hline Dibenzo(a,h)anthracene & 2 & 4.6 & 8.4 & 0.83 \\
\hline Fluoranthene & 2 & 46 & 82 & 10 \\
\hline C1-Fluoranthenes/pyrenes & 2 & 20 & 30 & 10 \\
\hline Fluorene & 2 & 4.1 & 5.5 & 2.7 \\
\hline C1-Fluorene & 0 & nd & nd & nd \\
\hline C2-Fluorene & 2 & 3.4 & 4.8 & 2.0 \\
\hline C3-Naphthalenes & 1 & 3.9 & 3.9 & 3.9 \\
\hline C4-Naphthalenes & 2 & 4.4 & 5.5 & 3.3 \\
\hline Perylene & 2 & 6.6 & 12 & 1.3 \\
\hline Phenanthrene & 2 & 21 & 35 & 7.6 \\
\hline C1-Phenanthrenes/anthracenes & 2 & 23 & 34 & 11 \\
\hline C2-Phenanthrenes/anthracenes & 2 & 20 & 24 & 16 \\
\hline C3-Phenanthrenes/anthracenes & 2 & 25 & 38 & 13 \\
\hline C4-Phenanthrenes/anthracenes & 2 & 6.3 & 7.1 & 5.5 \\
\hline Pyrene & 2 & 41 & 73 & 9.3 \\
\hline
\end{tabular}


Table 2.3. Summary of pesticide concentrations measured in suspended-sediment and bed-sediment samples from tributaries to the Anacostia River.

[ $\mu \mathrm{g} / \mathrm{kg}$, micrograms per kilogram; mg/kg, milligrams per kilogram; SS, suspended sediment; BS, bed sediment; nd, not detected]

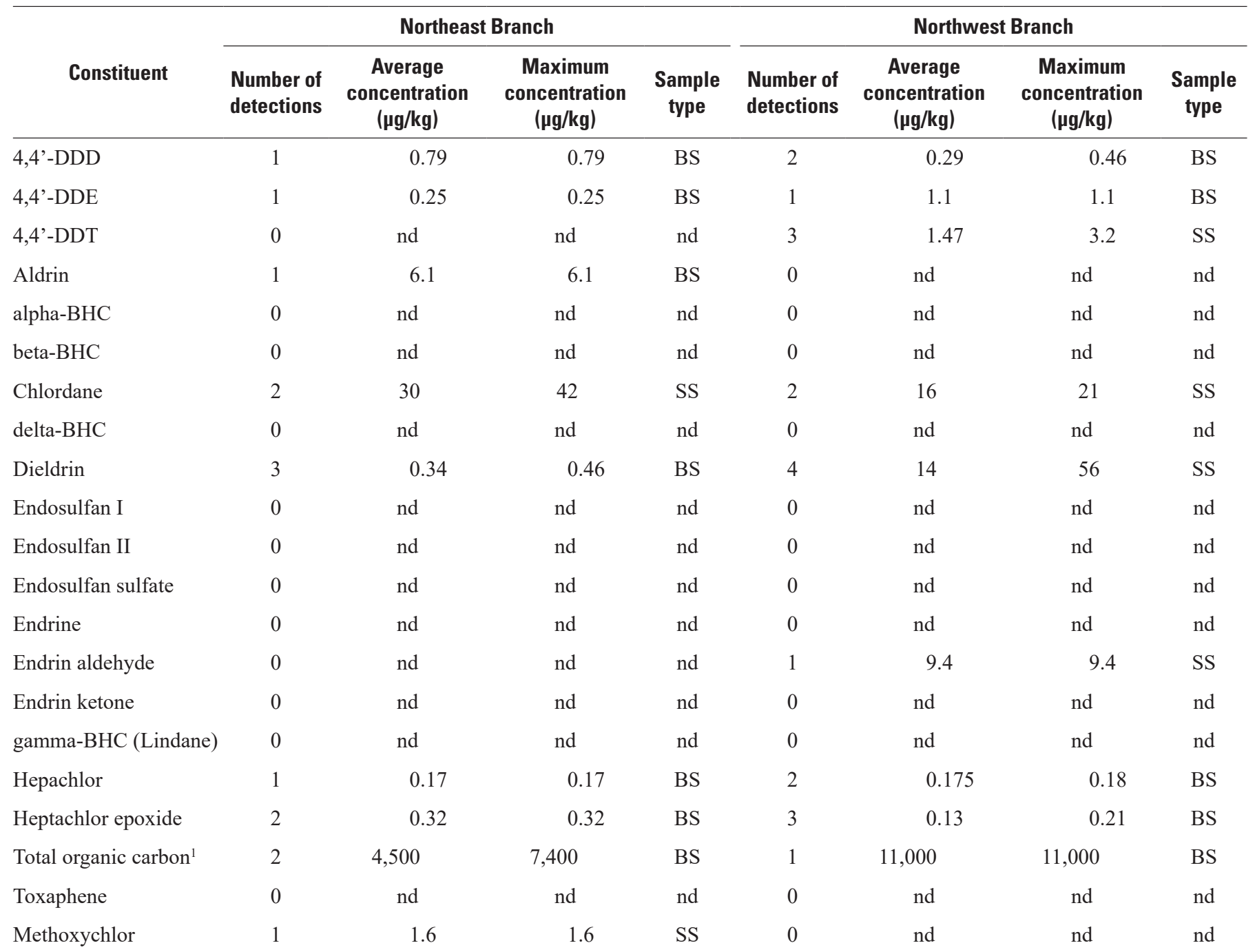


Table 2.3. Summary of pesticide concentrations measured in suspended-sediment and bed-sediment samples from tributaries to the Anacostia River.-Continued

$[\mu \mathrm{g} / \mathrm{kg}$, micrograms per kilogram; mg/kg, milligrams per kilogram; SS, suspended sediment; BS, bed sediment; nd, not detected]

\begin{tabular}{|c|c|c|c|c|c|c|c|c|}
\hline \multirow[b]{2}{*}{ Constituent } & \multicolumn{4}{|c|}{ Beaverdam Creek } & \multicolumn{4}{|c|}{ Watts Branch } \\
\hline & $\begin{array}{l}\text { Number of } \\
\text { detections }\end{array}$ & $\begin{array}{c}\text { Average } \\
\text { concentration } \\
(\mu \mathrm{g} / \mathrm{kg})\end{array}$ & $\begin{array}{c}\text { Maximum } \\
\text { concentration } \\
(\mu \mathrm{g} / \mathrm{kg})\end{array}$ & $\begin{array}{c}\text { Sample } \\
\text { type }\end{array}$ & $\begin{array}{l}\text { Number of } \\
\text { detections }\end{array}$ & $\begin{array}{c}\text { Average } \\
\text { concentration } \\
(\mu \mathrm{g} / \mathrm{kg})\end{array}$ & $\begin{array}{l}\text { Maximum } \\
\text { concentration } \\
(\mu \mathrm{g} / \mathrm{kg})\end{array}$ & $\begin{array}{c}\text { Sample } \\
\text { type }\end{array}$ \\
\hline 4,4'-DDD & 1 & 2.2 & 2.2 & BS & 3 & 1.4 & 1.8 & BS \\
\hline 4,4'-DDE & 1 & 1.5 & 1.5 & BS & 3 & 1.9 & 2.6 & BS \\
\hline 4,4'-DDT & 1 & 2.0 & 2.0 & BS & 4 & 37 & 140 & SS \\
\hline Aldrin & 0 & nd & nd & nd & 0 & nd & nd & nd \\
\hline alpha-BHC & 0 & nd & nd & nd & 0 & nd & nd & nd \\
\hline beta-BHC & 0 & nd & nd & nd & 0 & nd & nd & nd \\
\hline Chlordane & 3 & 43 & 70 & SS & 4 & 62 & 100 & SS \\
\hline delta-BHC & 0 & nd & nd & nd & 1 & 24 & 24 & SS \\
\hline Dieldrin & 1 & 0.39 & 0.39 & BS & 2 & 0.44 & 0.6 & BS \\
\hline Endosulfan I & 0 & nd & nd & nd & 0 & nd & nd & nd \\
\hline Endosulfan II & 0 & nd & nd & nd & 0 & nd & nd & nd \\
\hline Endosulfan sulfate & 0 & nd & nd & nd & 0 & nd & nd & nd \\
\hline Endrine & 0 & nd & nd & nd & 1 & 0.73 & 0.73 & $\mathrm{BS}$ \\
\hline Endrin aldehyde & 0 & nd & nd & nd & 2 & 49 & 98 & SS \\
\hline Endrin ketone & 0 & nd & nd & nd & 0 & nd & nd & nd \\
\hline gamma-BHC (Lindane) & 1 & 0.37 & 0.37 & BS & 0 & nd & nd & nd \\
\hline Heptachlor & 2 & 0.19 & 0.22 & $\mathrm{BS}$ & 0 & nd & nd & nd \\
\hline Heptachlor epoxide & 6 & 1.50 & 7.5 & SS & 2 & 0.21 & 0.31 & BS \\
\hline Total organic carbon ${ }^{1}$ & 2 & 5,350 & 9,100 & BS & 2 & 3,600 & 3,700 & $\mathrm{BS}$ \\
\hline Toxaphene & 0 & nd & nd & nd & 0 & nd & nd & nd \\
\hline Methoxychlor & 1 & 46 & 46 & $\mathrm{BS}$ & 1 & 103 & 103 & SS \\
\hline
\end{tabular}


Table 2.3. Summary of pesticide concentrations measured in suspended-sediment and bed-sediment samples from tributaries to the Anacostia River.-Continued

$[\mu \mathrm{g} / \mathrm{kg}$, micrograms per kilogram; mg/kg, milligrams per kilogram; SS, suspended sediment; BS, bed sediment; nd, not detected]

\begin{tabular}{|c|c|c|c|c|c|c|c|c|}
\hline \multirow[b]{2}{*}{ Constituent } & \multicolumn{4}{|c|}{ Hickey Run } & \multicolumn{4}{|c|}{ Nash Run } \\
\hline & $\begin{array}{l}\text { Number of } \\
\text { detections }\end{array}$ & $\begin{array}{c}\text { Average } \\
\text { concentration } \\
(\mu \mathrm{gg} / \mathrm{kg})\end{array}$ & $\begin{array}{c}\text { Maximum } \\
\text { concentration } \\
(\mu \mathrm{g} / \mathrm{kg})\end{array}$ & $\begin{array}{c}\text { Sample } \\
\text { type }\end{array}$ & $\begin{array}{l}\text { Number of } \\
\text { detections }\end{array}$ & $\begin{array}{c}\text { Average } \\
\text { concentration } \\
(\mu \mathrm{g} / \mathrm{kg})\end{array}$ & $\begin{array}{c}\text { Maximum } \\
\text { concentration } \\
(\mu \mathbf{g g} / \mathbf{k g})\end{array}$ & $\begin{array}{c}\text { Sample } \\
\text { type }\end{array}$ \\
\hline 4,4'-DDD & 5 & 2.9 & 4.6 & BS & 1 & 0.23 & 0.23 & BS \\
\hline 4,4'-DDE & 6 & 3.5 & 5.5 & BS & 0 & nd & nd & nd \\
\hline 4,4'-DDT & 4 & 7.6 & 23 & BS & 0 & nd & nd & nd \\
\hline Aldrin & 0 & nd & nd & nd & 0 & nd & nd & nd \\
\hline alpha-BHC & 0 & nd & nd & nd & 0 & nd & nd & nd \\
\hline beta-BHC & 0 & nd & nd & nd & 0 & nd & nd & nd \\
\hline Chlordane & 3 & 27 & 40 & SS & 0 & nd & nd & nd \\
\hline delta-BHC & 0 & nd & nd & nd & 0 & nd & nd & nd \\
\hline Dieldrin & 4 & 0.68 & 0.89 & BS & 1 & 0.041 & 0.041 & BS \\
\hline Endosulfan I & 0 & nd & nd & nd & 0 & nd & nd & nd \\
\hline Endosulfan II & 0 & nd & nd & nd & 0 & nd & nd & nd \\
\hline Endosulfan sulfate & 0 & nd & nd & nd & 0 & nd & nd & nd \\
\hline Endrine & 1 & 0.63 & 0.63 & BS & 0 & nd & nd & nd \\
\hline Endrin aldehyde & 1 & 0.15 & 0.15 & BS & 0 & nd & nd & nd \\
\hline Endrin ketone & 0 & nd & nd & nd & 0 & nd & nd & nd \\
\hline gamma-BHC (Lindane) & 0 & nd & nd & nd & 0 & nd & nd & nd \\
\hline Heptachlor & 1 & 0.13 & 0.13 & BS & 0 & nd & nd & nd \\
\hline Heptachlor epoxide & 3 & 0.31 & 0.52 & BS & 0 & nd & nd & nd \\
\hline Total organic carbon ${ }^{1}$ & 3 & 8,000 & 13,000 & BS & 1 & 5,700 & 5,700 & BS \\
\hline Toxaphene & 0 & nd & nd & nd & 0 & nd & nd & nd \\
\hline Methoxychlor & 0 & nd & nd & nd & 0 & nd & nd & nd \\
\hline
\end{tabular}


Table 2.3. Summary of pesticide concentrations measured in suspended-sediment and bed-sediment samples from tributaries to the Anacostia River.-Continued

[ $\mu \mathrm{g} / \mathrm{kg}$, micrograms per kilogram; mg/kg, milligrams per kilogram; SS, suspended sediment; BS, bed sediment; nd, not detected]

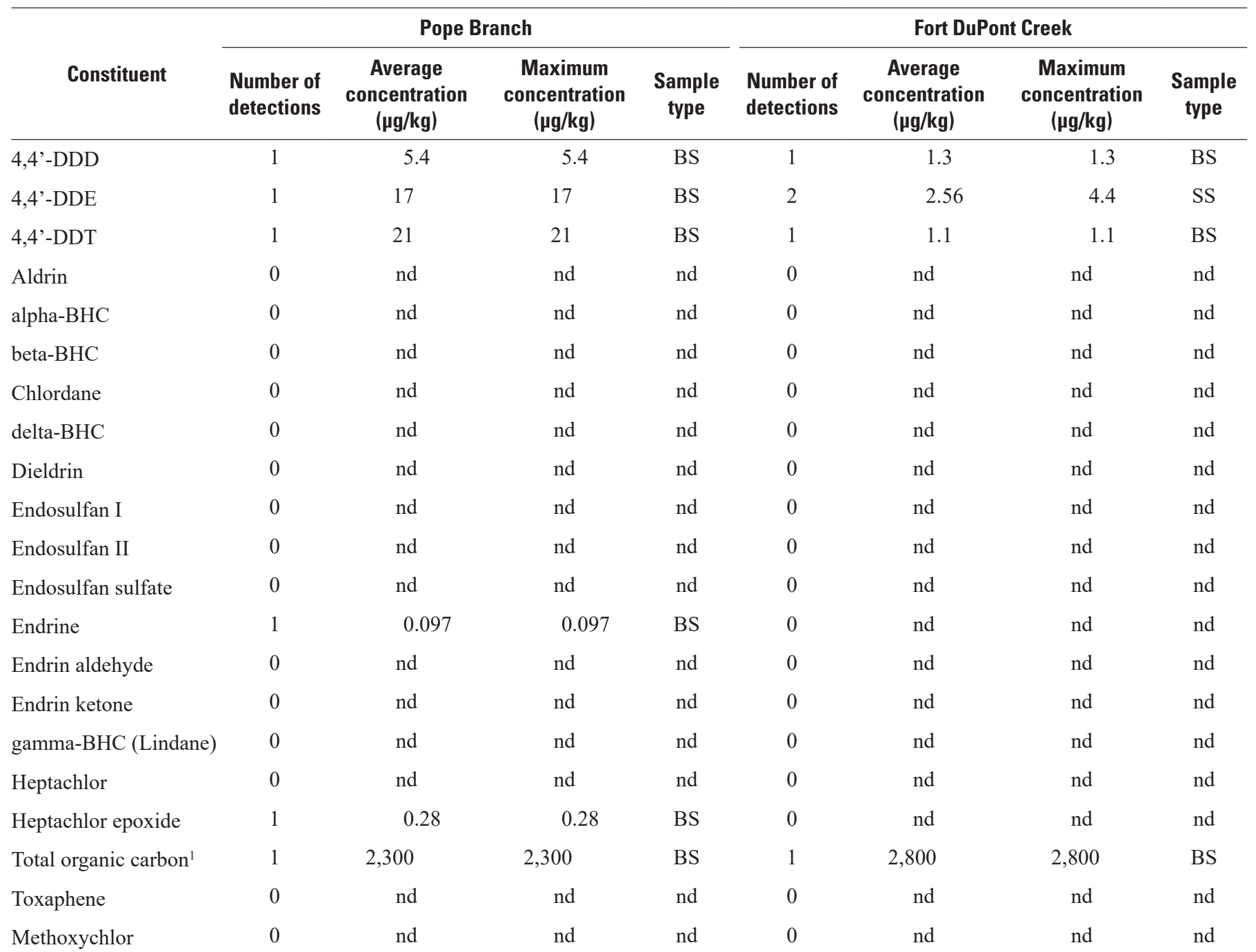


Table 2.3. Summary of pesticide concentrations measured in suspended-sediment and bed-sediment samples from tributaries to the Anacostia River.-Continued

$[\mu \mathrm{g} / \mathrm{kg}$, micrograms per kilogram; mg/kg, milligrams per kilogram; SS, suspended sediment; BS, bed sediment; nd, not detected]

\begin{tabular}{|c|c|c|c|c|}
\hline \multirow[b]{2}{*}{ Constituent } & \multicolumn{4}{|c|}{ Fort Stanton Creek } \\
\hline & $\begin{array}{l}\text { Number of } \\
\text { detections }\end{array}$ & $\begin{array}{c}\text { Average } \\
\text { concentration } \\
(\mu \mathrm{g} / \mathrm{kg})\end{array}$ & $\begin{array}{c}\text { Maximum } \\
\text { concentration } \\
(\mu \mathbf{g} / \mathbf{k g})\end{array}$ & $\begin{array}{c}\text { Sample } \\
\text { type }\end{array}$ \\
\hline 4,4'-DDD & 1 & 0.41 & 0.41 & BS \\
\hline 4,4'-DDE & 1 & 0.36 & 0.36 & BS \\
\hline 4,4'-DDT & 1 & 0.62 & 0.62 & BS \\
\hline Aldrin & 0 & nd & nd & nd \\
\hline alpha-BHC & 0 & nd & nd & nd \\
\hline beta-BHC & 0 & nd & nd & nd \\
\hline Chlordane & 0 & nd & nd & nd \\
\hline delta-BHC & 0 & nd & nd & nd \\
\hline Dieldrin & 0 & nd & nd & nd \\
\hline Endosulfan I & 0 & nd & nd & nd \\
\hline Endosulfan II & 0 & nd & nd & nd \\
\hline Endosulfan sulfate & 0 & nd & nd & nd \\
\hline Endrine & 0 & nd & nd & nd \\
\hline Endrin aldehyde & 0 & nd & nd & nd \\
\hline Endrin ketone & 0 & nd & nd & nd \\
\hline gamma-BHC (Lindane) & 0 & nd & nd & nd \\
\hline Heptachlor & 0 & nd & nd & nd \\
\hline Heptachlor epoxide & 0 & nd & nd & nd \\
\hline Total organic carbon ${ }^{1}$ & 1 & 4,600 & 4,600 & BS \\
\hline Toxaphene & 0 & nd & nd & nd \\
\hline Methoxychlor & 0 & nd & nd & nd \\
\hline
\end{tabular}

${ }^{1}$ Total organic carbon in $\mathrm{mg} / \mathrm{kg}$. 
Table 2.4. Summary of metal concentrations in suspended-sediment and bed-sediment samples from tributaries to the Anacostia River.

[mg/kg, milligrams per kilogram; n, number of samples]

\begin{tabular}{|c|c|c|c|c|c|c|c|c|c|}
\hline \multirow{3}{*}{ Metal } & \multicolumn{3}{|c|}{ Northeast Branch } & \multicolumn{3}{|c|}{ Northwest Branch } & \multicolumn{3}{|c|}{ Beaverdam Creek } \\
\hline & \multicolumn{3}{|c|}{$\begin{array}{l}\text { Suspended sediment, } \mathrm{n}=0 \\
\text { bed sediment, } \mathrm{n}=3\end{array}$} & \multicolumn{3}{|c|}{$\begin{array}{l}\text { Suspended sediment, } \mathrm{n}=0 \text {; } \\
\text { bed sediment, } \mathrm{n}=3\end{array}$} & \multicolumn{3}{|c|}{$\begin{array}{l}\text { Suspended sediment, } \mathrm{n}=2 ; \\
\text { bed sediment, } \mathrm{n}=5\end{array}$} \\
\hline & Average & Maximum & Minimum & Average & Maximum & Minimum & Average & Maximum & Minimum \\
\hline Aluminum & 790 & 980 & 620 & 2,000 & 2,600 & 1,600 & 3,900 & 15,000 & 790 \\
\hline Barium & 8.2 & 11 & 6.5 & 14 & 23 & 8.1 & 80 & 420 & 7.0 \\
\hline Beryllium & 0.19 & 0.20 & 0.17 & 0.24 & 0.25 & 0.22 & 0.55 & 1.1 & 0.24 \\
\hline Cadmium & 0.048 & 0.064 & 0.039 & 0.050 & 0.077 & 0.027 & 1.5 & 8.6 & 0.13 \\
\hline Calcium & 3,500 & 4,600 & 2,200 & 780 & 1,000 & 670 & 3,900 & 18,000 & 420 \\
\hline Iron & 4,900 & 6,400 & 4,000 & 6,300 & 8,500 & 4,500 & 17,000 & 48,000 & 7,800 \\
\hline Lead & 4.1 & 6.1 & 2.7 & 9.4 & 20 & 3.3 & 140 & 790 & 10 \\
\hline Magnesium & 2,000 & 2,700 & 1,100 & 1,700 & 2,000 & 1,400 & 2,100 & 8,900 & 390 \\
\hline Manganese & 99 & 140 & 66 & 75 & 86 & 61 & 220 & 650 & 58 \\
\hline Mercury & 0.0080 & 0.01 & 0.0061 & 0.0052 & 0.0055 & 0.0045 & 0.33 & 2.2 & 0.0070 \\
\hline Nickel & 5.2 & 6.9 & 4.3 & 11 & 13 & 9.1 & 25 & 100 & 4.1 \\
\hline Potassium & 130 & 180 & 73 & 490 & 720 & 330 & 590 & 1,200 & 230 \\
\hline Selenium & 0.11 & 0.16 & 0.069 & 0.16 & 0.34 & 0.072 & 0.42 & 1.200 & 0.092 \\
\hline Silver & 0.017 & 0.027 & 0.0089 & 0.014 & 0.025 & 0.008 & 0.61 & 3.900 & 0.0090 \\
\hline
\end{tabular}


Table 2.4. Summary of metal concentrations in suspended-sediment and bed-sediment samples from tributaries to the Anacostia River.-Continued

[mg/kg, milligrams per kilogram; n, number of samples]

\begin{tabular}{|c|c|c|c|c|c|c|c|c|c|}
\hline \multirow{3}{*}{ Metal } & \multicolumn{3}{|c|}{ Watts Branch } & \multicolumn{3}{|c|}{ Hickey Run } & \multicolumn{3}{|c|}{ Pope Branch } \\
\hline & \multicolumn{3}{|c|}{$\begin{array}{l}\text { Suspended sediment, } n=3 \\
\text { bed sediment, } n=3\end{array}$} & \multicolumn{3}{|c|}{$\begin{array}{l}\text { Suspended sediment, } \mathrm{n}=1 \\
\text { bed sediment, } \mathrm{n}=5\end{array}$} & \multicolumn{3}{|c|}{$\begin{array}{l}\text { Suspended sediment, } \mathrm{n}=1 \\
\text { bed sediment, } \mathrm{n}=1\end{array}$} \\
\hline & Average & Maximum & Minimum & Average & Maximum & Minimum & Average & Maximum & Minimum \\
\hline Aluminum & 5,500 & 14,000 & 1,100 & 3,300 & 6,700 & 1,600 & 2,100 & 2,600 & 1,500 \\
\hline Barium & 79 & 140 & 15 & 54 & 110 & 32 & 31 & 53 & 9.8 \\
\hline Beryllium & 0.90 & 1.6 & 0.32 & 0.30 & 0.48 & 0.099 & 0.22 & 0.24 & 0.20 \\
\hline Cadmium & 1.1 & 3.0 & 0.11 & 0.29 & 0.89 & 0.11 & 0.18 & 0.26 & 0.10 \\
\hline Calcium & 4,500 & 7,900 & 1,200 & 26,000 & 40,000 & 12,000 & 2,000 & 2,200 & 1,800 \\
\hline Iron & 23,000 & 45,000 & 8,700 & 16,000 & 24,000 & 11,000 & 20,000 & 29,000 & 11,000 \\
\hline Lead & 81 & 150 & 12 & 65 & 130 & 16 & 16 & 17 & 15 \\
\hline Magnesium & 2,500 & 4,100 & 910 & 12,000 & 21,000 & 5,600 & 8,900 & 17,000 & 800 \\
\hline Manganese & 511 & 1,300 & 93 & 310 & 530 & 190 & 780 & 1,500 & 91 \\
\hline Mercury & 0.096 & 0.24 & 0.0076 & 0.030 & 0.14 & 0.0068 & 0.021 & 0.031 & 0.01 \\
\hline Nickel & 33 & 63 & 9.4 & 29 & 35 & 17 & 110 & 210 & 6.7 \\
\hline Potassium & 780 & 1,700 & 230 & 370 & 1,100 & 180 & 270 & 440 & 99 \\
\hline Selenium & 0.96 & 2.1 & 0.097 & 0.25 & 0.56 & 0.13 & 0.60 & 0.99 & 0.21 \\
\hline Silver & 0.24 & 0.54 & 0.0099 & 0.11 & 0.50 & 0.022 & 0.062 & 0.11 & 0.013 \\
\hline
\end{tabular}


Table 2.4. Summary of metal concentrations in suspended-sediment and bed-sediment samples from tributaries to the Anacostia River.-Continued

[mg/kg, milligrams per kilogram; n, number of samples]

\begin{tabular}{|c|c|c|c|c|c|}
\hline \multirow{4}{*}{ Metal } & \multirow{2}{*}{$\begin{array}{c}\text { Nash Run } \\
\begin{array}{c}\text { Suspended sediment, } \mathrm{n}=0 ; \\
\text { bed sediment, } \mathrm{n}=1\end{array} \\
\end{array}$} & \multicolumn{3}{|c|}{ Fort DuPont Creek } & \multirow{4}{*}{$\begin{array}{c}\text { Fort Stanton Creek } \\
\begin{array}{c}\text { Suspended sediment, } \mathrm{n}=0 ; \\
\text { bed sediment, } \mathrm{n}=1\end{array} \\
\text { Concentration }(\mathbf{m g} / \mathbf{k g})\end{array}$} \\
\hline & & Susp & $\begin{array}{l}\text { ended sedimer } \\
\text { ed sediment, }\end{array}$ & $\begin{array}{l}n t, n=1 ; \\
n=1\end{array}$ & \\
\hline & \multirow{2}{*}{ Concentration (mg/kg) } & \multicolumn{3}{|c|}{ Concentration (mg/kg) } & \\
\hline & & Average & Maximum & Minimum & \\
\hline Aluminum & 980 & 2,400 & 4,000 & 800 & 1,700 \\
\hline Antimony & 0.11 & 0.29 & 0.51 & 0.069 & 0.23 \\
\hline Arsenic & 2.6 & 3.5 & 4.6 & 2.4 & 5.9 \\
\hline Barium & 11 & 39 & 70 & 8.2 & 19 \\
\hline Beryllium & 0.48 & 0.51 & 0.77 & 0.24 & 0.64 \\
\hline Cadmium & 0.06 & 0.13 & 0.23 & 0.026 & 0.19 \\
\hline Calcium & 330 & 820 & 1,400 & 250 & 480 \\
\hline Chromium & 6.8 & 13 & 16 & 9.5 & 24 \\
\hline Cobalt & 6.6 & 9.6 & 18 & 1.2 & 4.9 \\
\hline Copper & 5.6 & 11 & 20 & 2.5 & 10 \\
\hline Iron & 12,000 & 16,000 & 20,000 & 12,000 & 34,000 \\
\hline Lead & 7.3 & 16. & 29 & 3.1 & 8.9 \\
\hline Magnesium & 500 & 410 & 690 & 120 & 210 \\
\hline Manganese & 120 & 250 & 470 & 38 & 240 \\
\hline Mercury & 0.019 & 0.060 & 0.11 & 0.0099 & 0.0096 \\
\hline Nickel & 8.9 & 7.8 & 14 & 1.5 & 6.0 \\
\hline Potassium & 96 & 290 & 510 & 74 & 150 \\
\hline Selenium & 0.21 & 0.50 & 0.76 & 0.23 & 0.41 \\
\hline Silver & 0.016 & 0.26 & 0.52 & 0.0084 & 0.011 \\
\hline Sodium & 33 & 39 & 57 & 21 & 26 \\
\hline Thallium & 0.025 & 0.055 & 0.10 & 0.011 & 0.024 \\
\hline Vanadium & 14 & 21 & 31 & 11 & 67 \\
\hline Zinc & 26 & 37 & 66 & 7.3 & 42 \\
\hline
\end{tabular}

${ }^{1}$ Number of suspended- and bed-sediment samples used to calculate average concentrations. 


\section{Appendix 3. Datasets used to model suspended sediment in tributaries to the Anacostia River, 2017.}

This appendix presents the suspended-sediment data and discharge and turbidity data taken from continuous gages and water-quality sondes deployed on the tributaries to the Anacostia River. These data were used to develop the linear regression equations used to predict suspended-sediment concentrations and loads from continuously measured discharge and turbidity records. These data are publicly available in the ScienceBase repository as a U.S. Geological Survey data release (Wilson, 2019). 
Table 3.1. Suspended-sediment data used to develop linear regression models.

[ft, feet; $\mathrm{ft}^{3} / \mathrm{s}$, cubic feet per second; FNU, Formazin Nephelometric Units; SSC, suspended-sediment concentration; mg/L, milligrams per liter; nr, not measured; average discharge and average turbidity are calculated from measurements beginning 15 minutes before through 15 minutes after the collection time reported for the suspended-sediment sample]

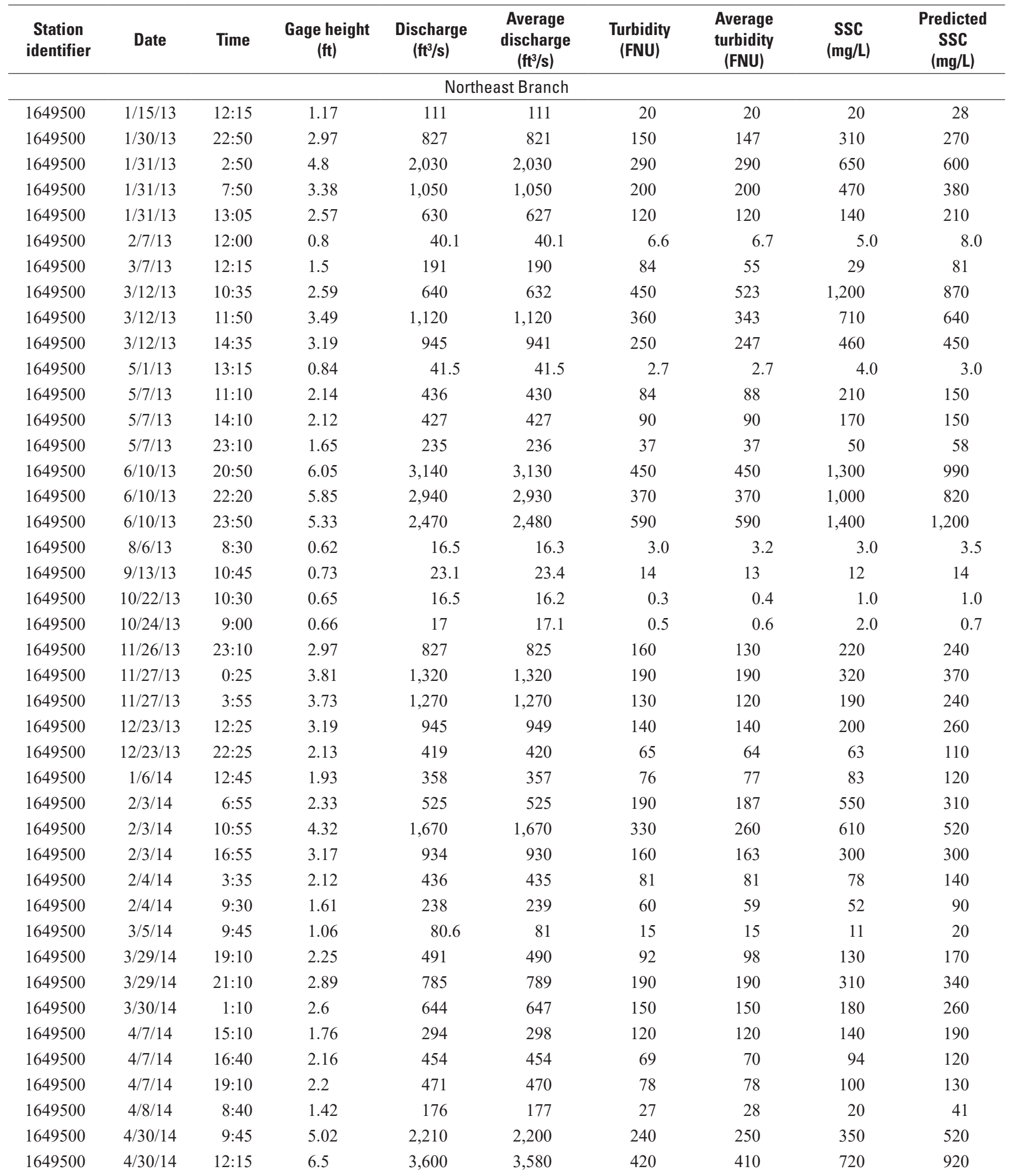


Table 3.1. Suspended-sediment data used to develop linear regression models.-Continued

[ft, feet; $\mathrm{ft}^{3} / \mathrm{s}$, cubic feet per second; FNU, Formazin Nephelometric Units; SSC, suspended-sediment concentration; mg/L, milligrams per liter; nr, not measured; average discharge and average turbidity are calculated from measurements beginning 15 minutes before through 15 minutes after the collection time reported for the suspended-sediment sample]

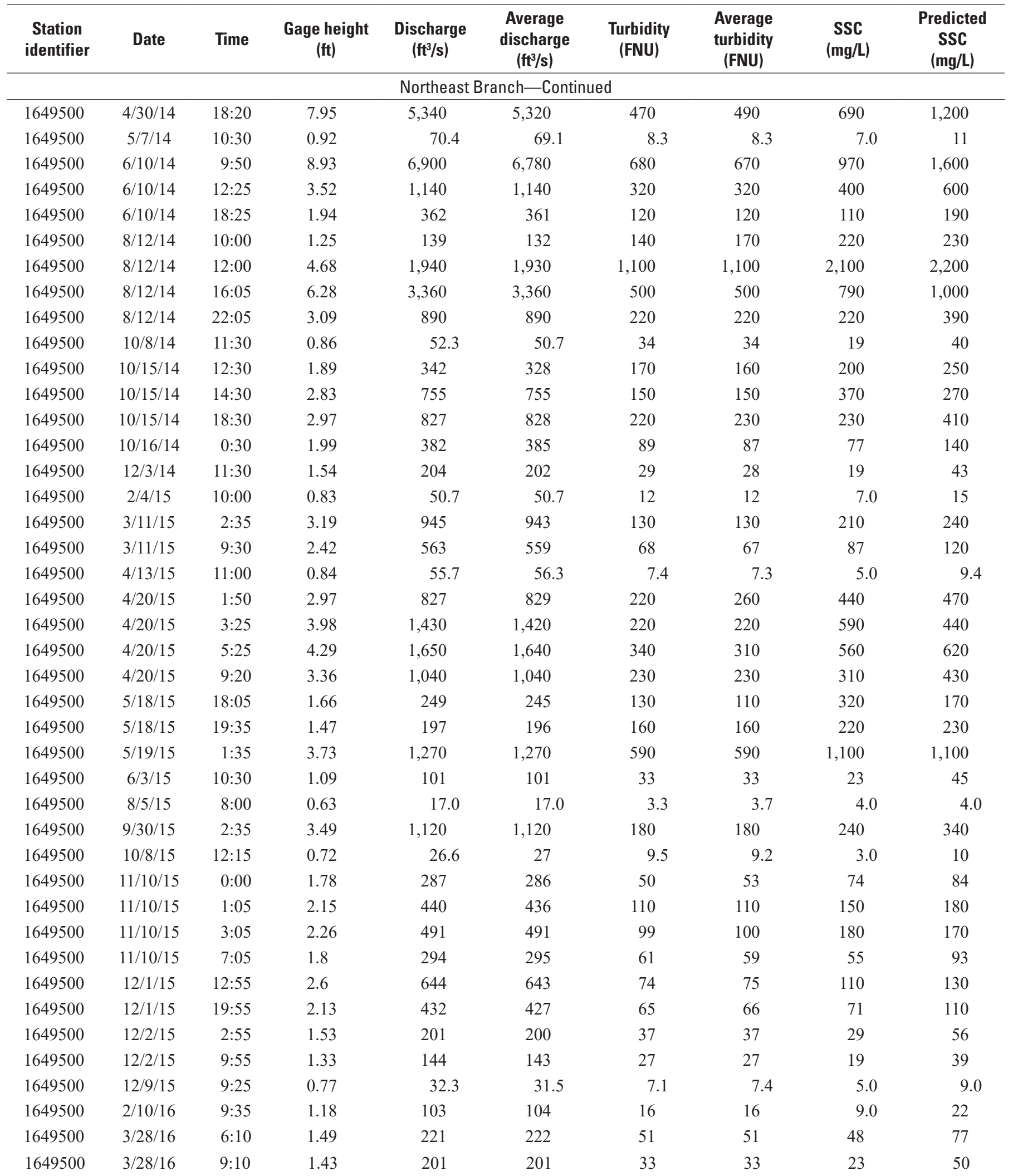


Table 3.1. Suspended-sediment data used to develop linear regression models.—Continued

[ft, feet; $\mathrm{ft}^{3} / \mathrm{s}$, cubic feet per second; FNU, Formazin Nephelometric Units; SSC, suspended-sediment concentration; mg/L, milligrams per liter; nr, not measured; average discharge and average turbidity are calculated from measurements beginning 15 minutes before through 15 minutes after the collection time reported for the suspended-sediment sample]

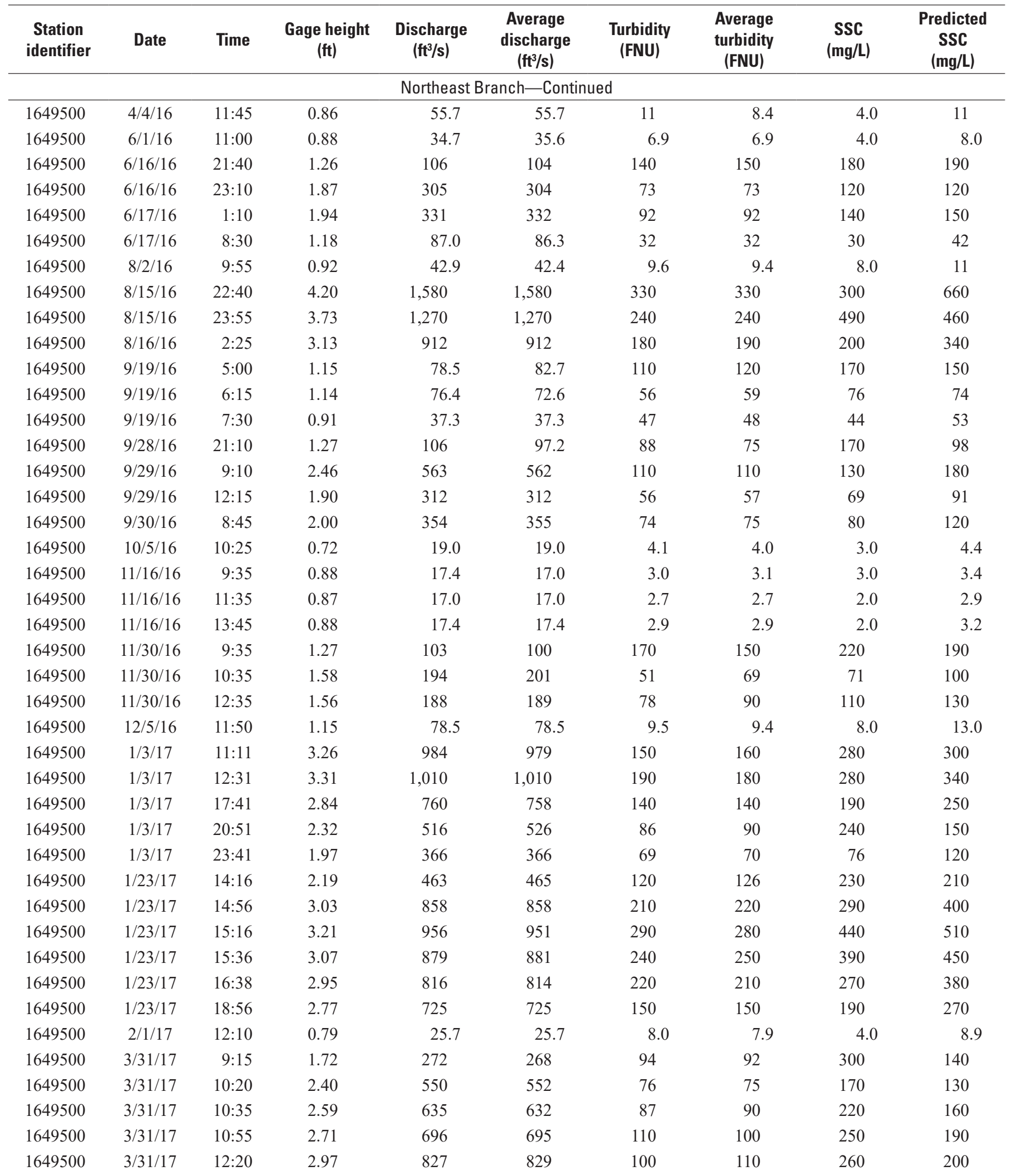


Table 3.1. Suspended-sediment data used to develop linear regression models. - Continued

[ft, feet; $\mathrm{ft}^{3} / \mathrm{s}$, cubic feet per second; FNU, Formazin Nephelometric Units; SSC, suspended-sediment concentration; mg/L, milligrams per liter; nr, not measured; average discharge and average turbidity are calculated from measurements beginning 15 minutes before through 15 minutes after the collection time reported for the suspended-sediment sample]

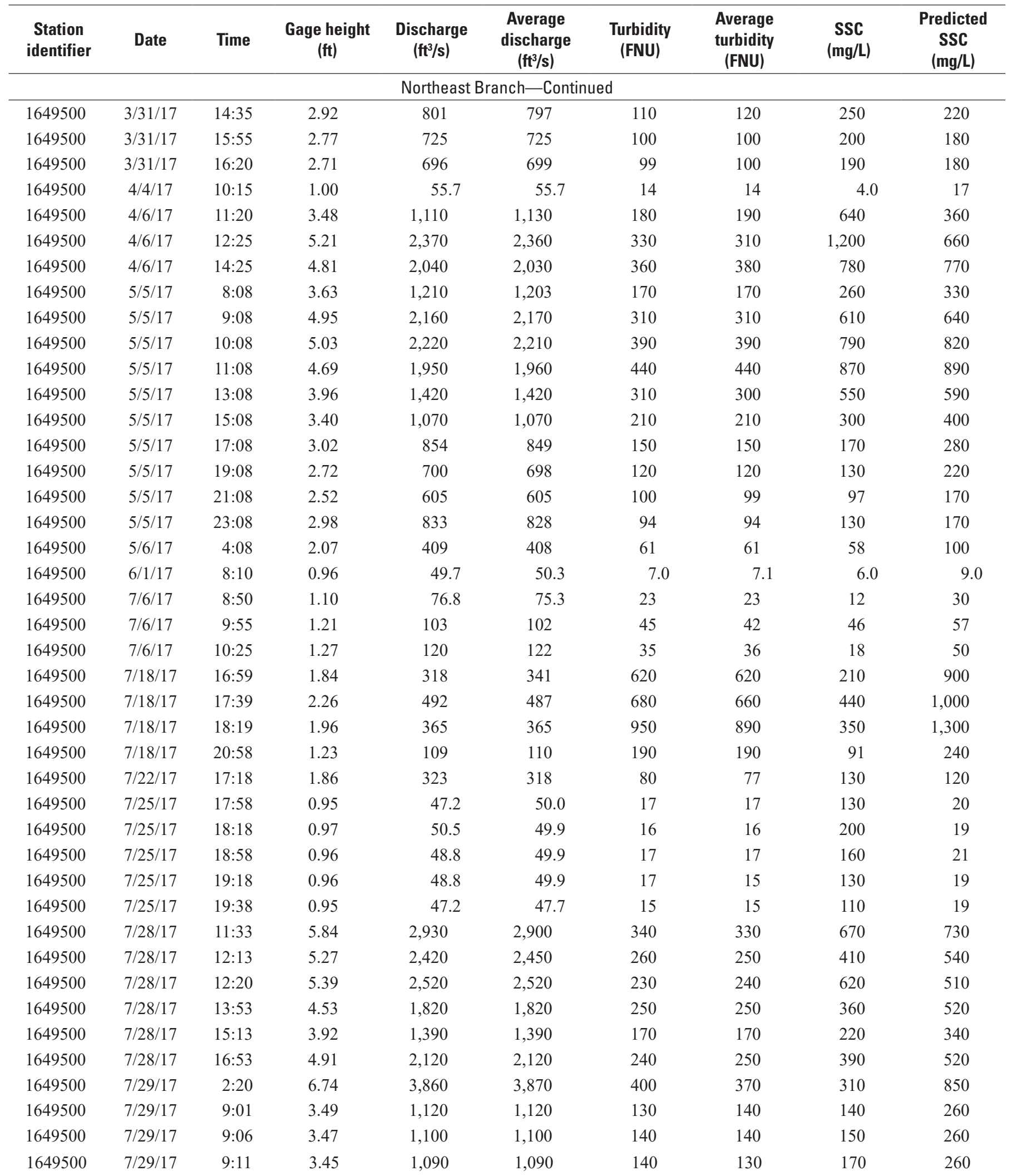


Table 3.1. Suspended-sediment data used to develop linear regression models. - Continued

[ft, feet; $\mathrm{ft}^{3} / \mathrm{s}$, cubic feet per second; FNU, Formazin Nephelometric Units; SSC, suspended-sediment concentration; mg/L, milligrams per liter; nr, not measured; average discharge and average turbidity are calculated from measurements beginning 15 minutes before through 15 minutes after the collection time reported for the suspended-sediment sample]

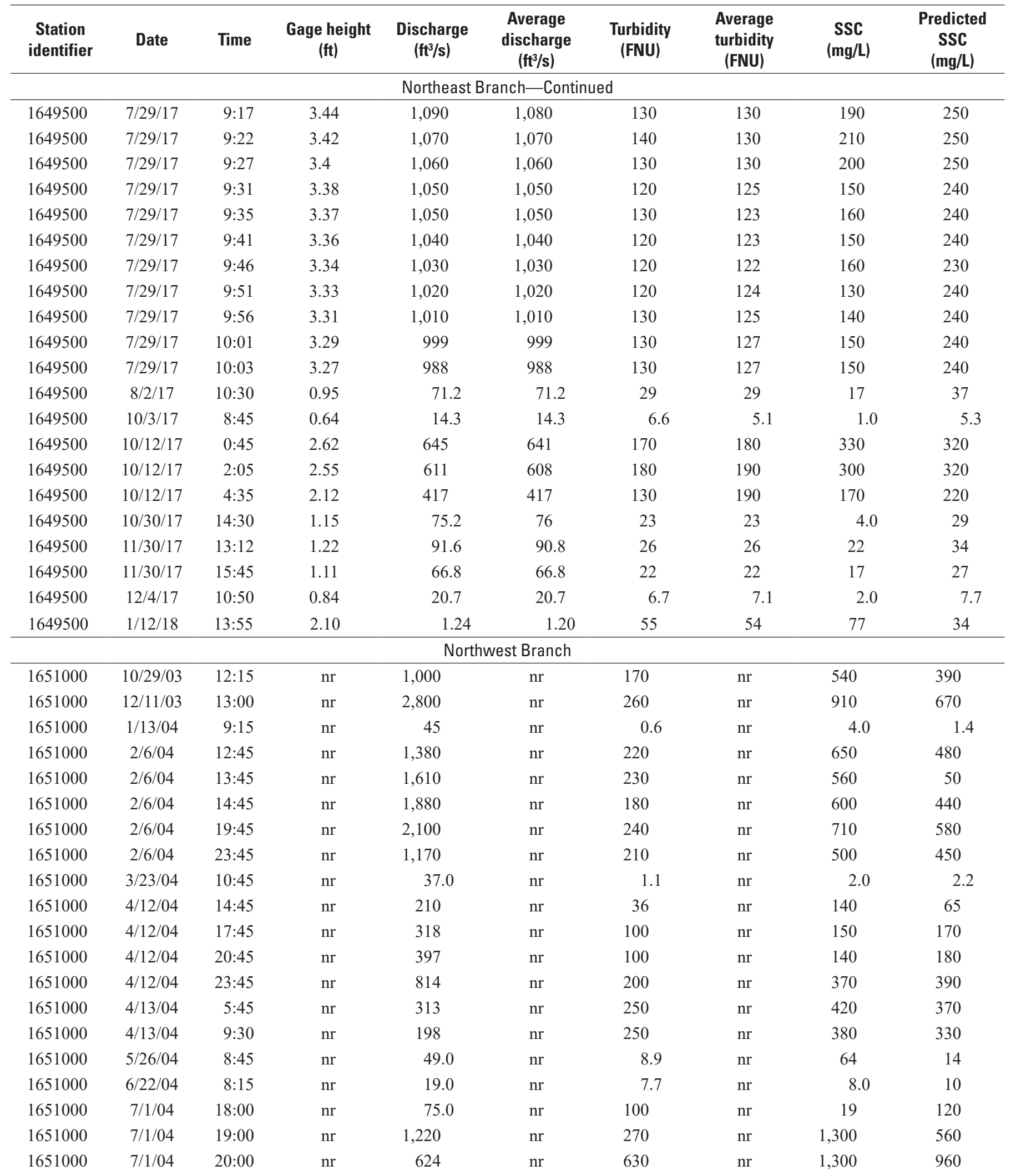


Table 3.1. Suspended-sediment data used to develop linear regression models. - Continued

[ft, feet; $\mathrm{ft}^{3} / \mathrm{s}$, cubic feet per second; FNU, Formazin Nephelometric Units; SSC, suspended-sediment concentration; mg/L, milligrams per liter; nr, not measured; average discharge and average turbidity are calculated from measurements beginning 15 minutes before through 15 minutes after the collection time reported for the suspended-sediment sample]

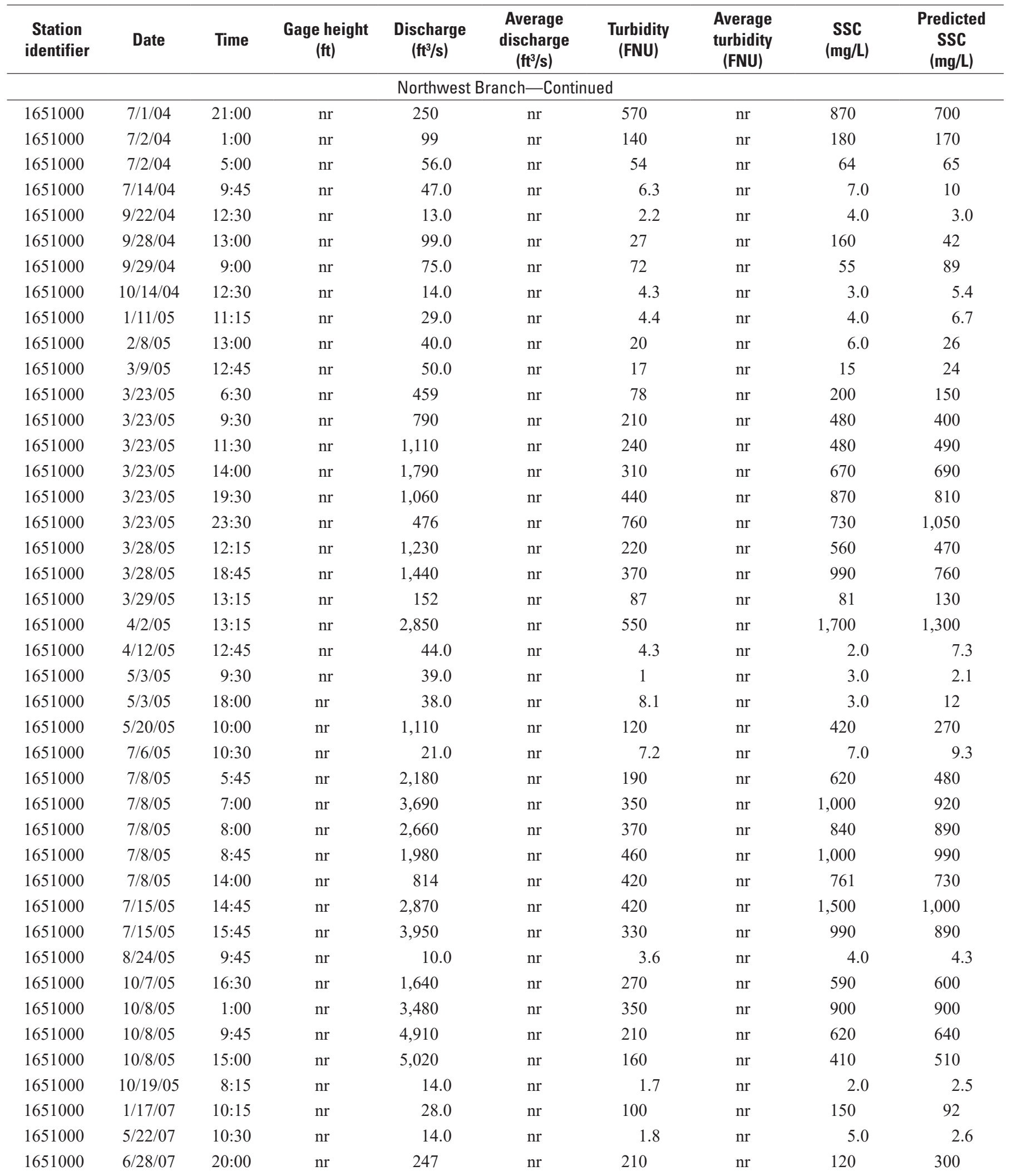


Table 3.1. Suspended-sediment data used to develop linear regression models. - Continued

[ft, feet; $\mathrm{ft}^{3} / \mathrm{s}$, cubic feet per second; FNU, Formazin Nephelometric Units; SSC, suspended-sediment concentration; mg/L, milligrams per liter; nr, not measured; average discharge and average turbidity are calculated from measurements beginning 15 minutes before through 15 minutes after the collection time reported for the suspended-sediment sample]

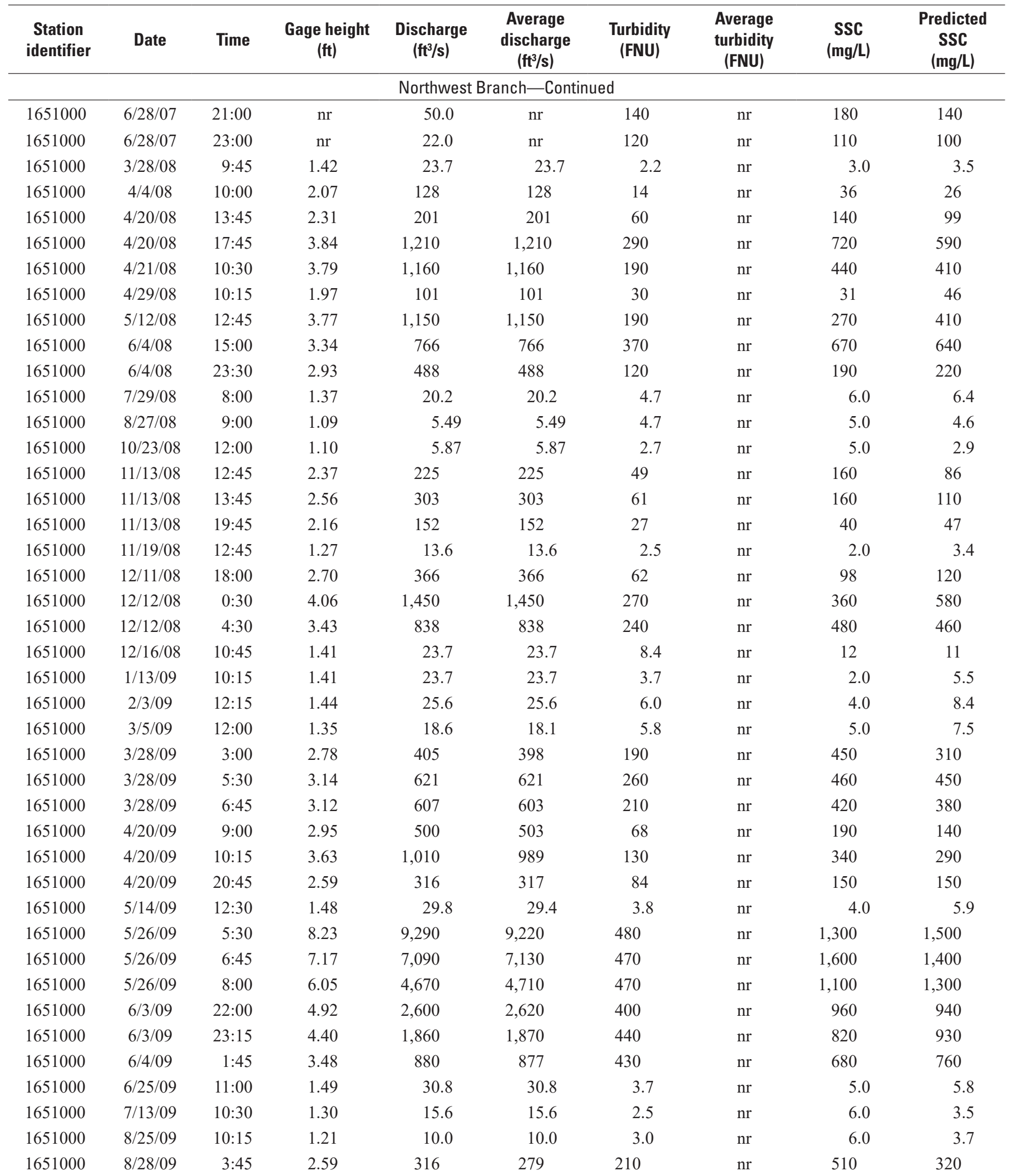


Table 3.1. Suspended-sediment data used to develop linear regression models. - Continued

[ft, feet; $\mathrm{ft}^{3} / \mathrm{s}$, cubic feet per second; FNU, Formazin Nephelometric Units; SSC, suspended-sediment concentration; mg/L, milligrams per liter; nr, not measured; average discharge and average turbidity are calculated from measurements beginning 15 minutes before through 15 minutes after the collection time reported for the suspended-sediment sample]

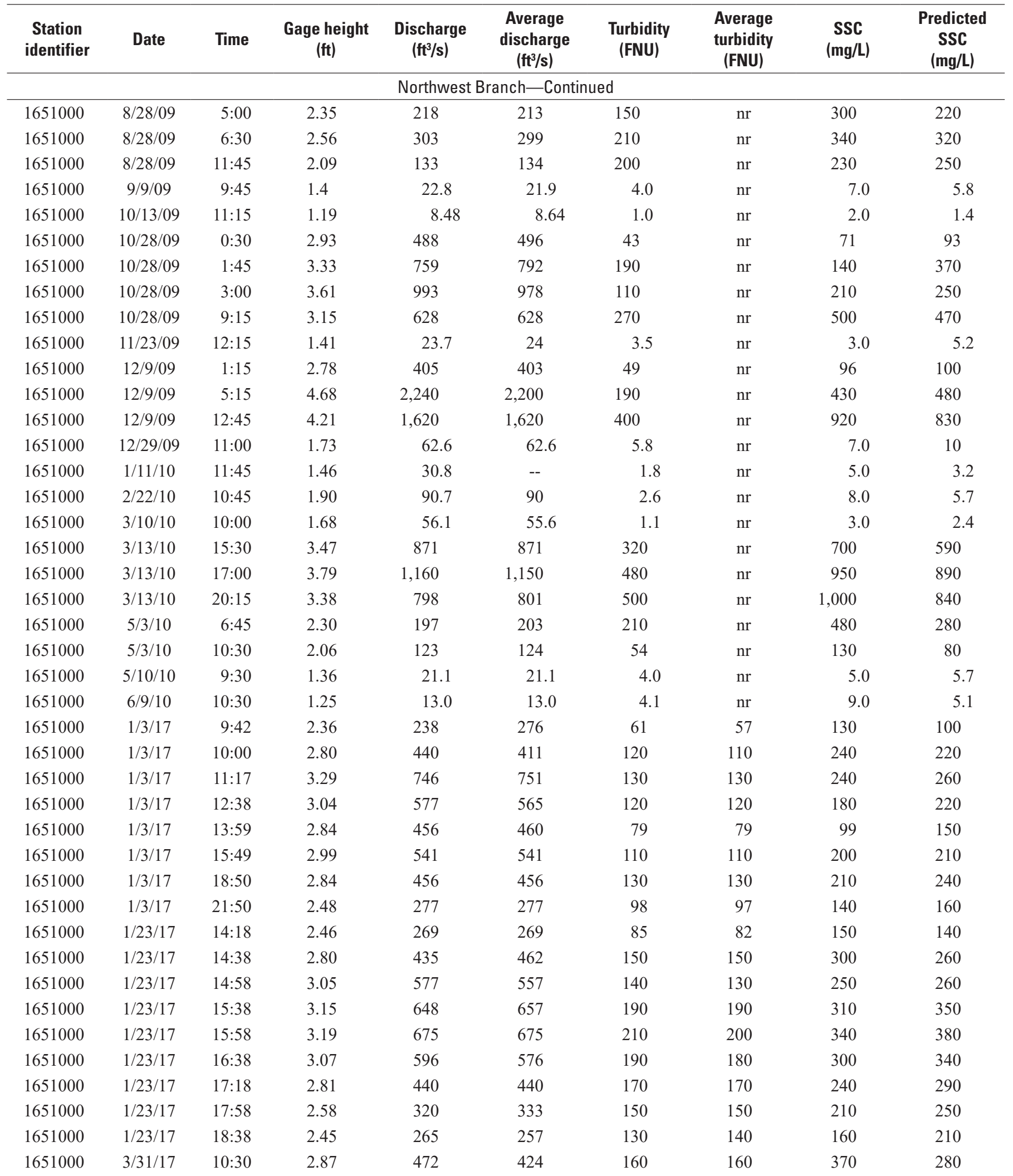


Table 3.1. Suspended-sediment data used to develop linear regression models.—Continued

[ft, feet; $\mathrm{ft}^{3} / \mathrm{s}$, cubic feet per second; FNU, Formazin Nephelometric Units; SSC, suspended-sediment concentration; mg/L, milligrams per liter; nr, not measured; average discharge and average turbidity are calculated from measurements beginning 15 minutes before through 15 minutes after the collection time reported for the suspended-sediment sample]

\begin{tabular}{|c|c|c|c|c|c|c|c|c|c|}
\hline $\begin{array}{l}\text { Station } \\
\text { identifier }\end{array}$ & Date & Time & $\begin{array}{c}\text { Gage height } \\
\text { (ft) }\end{array}$ & $\begin{array}{c}\text { Discharge } \\
\left(\mathrm{ft}^{3} / \mathrm{s}\right)\end{array}$ & $\begin{array}{c}\text { Average } \\
\text { discharge } \\
\left(\mathrm{ft}^{3} / \mathrm{s}\right)\end{array}$ & $\begin{array}{c}\text { Turbidity } \\
\text { (FNU) }\end{array}$ & $\begin{array}{c}\text { Average } \\
\text { turbidity } \\
\text { (FNU) }\end{array}$ & $\begin{array}{c}\text { SSC } \\
(\mathrm{mg} / \mathrm{L})\end{array}$ & $\begin{array}{c}\text { Predicted } \\
\text { SSC } \\
\text { (mg/L) }\end{array}$ \\
\hline \multicolumn{10}{|c|}{ Northwest Branch-Continued } \\
\hline 1651000 & $3 / 31 / 17$ & $10: 50$ & 2.94 & 512 & 512 & 190 & 190 & 400 & 320 \\
\hline 1651000 & $3 / 31 / 17$ & $11: 10$ & 3.05 & 577 & 581 & 220 & 210 & 470 & 380 \\
\hline 1651000 & $3 / 31 / 17$ & $12: 10$ & 3.13 & 635 & 633 & 160 & 160 & 350 & 300 \\
\hline 1651000 & $3 / 31 / 17$ & $15: 10$ & 2.91 & 494 & 490 & 160 & 160 & 300 & 280 \\
\hline 1651000 & $5 / 5 / 17$ & $8: 45$ & 4.53 & 2,030 & 2,020 & 210 & 220 & 510 & 510 \\
\hline 1651000 & $5 / 5 / 17$ & $11: 00$ & 3.95 & 1,330 & 1,400 & 340 & 350 & 660 & 690 \\
\hline 1651000 & $5 / 5 / 17$ & $12: 30$ & 3.88 & 1,250 & 1,230 & 420 & 430 & 840 & 820 \\
\hline 1651000 & $5 / 5 / 17$ & $14: 00$ & 3.70 & 1,080 & 1,090 & 520 & 650 & 1,000 & 940 \\
\hline 1651000 & $5 / 25 / 17$ & $1: 28$ & 2.08 & 148 & 146 & 41 & 41 & 66 & 66 \\
\hline 1651000 & $5 / 25 / 17$ & $3: 08$ & 2.11 & 156 & 155 & 33 & 32 & 42 & 55 \\
\hline 1651000 & $5 / 25 / 17$ & $6: 08$ & 2.31 & 220 & 212 & 49 & 49 & 81 & 85 \\
\hline 1651000 & $5 / 25 / 17$ & $6: 48$ & 2.49 & 290 & 290 & 56 & 58 & 110 & 100 \\
\hline 1651000 & $5 / 25 / 17$ & 7:08 & 2.60 & 339 & 348 & 76 & 75 & 130 & 140 \\
\hline 1651000 & $5 / 25 / 17$ & $7: 28$ & 2.71 & 392 & 384 & 84 & 82 & 160 & 160 \\
\hline 1651000 & $5 / 25 / 17$ & $7: 48$ & 2.74 & 408 & 408 & 97 & 97 & 180 & 180 \\
\hline 1651000 & $7 / 28 / 17$ & $11: 37$ & 4.19 & 1,560 & 1,560 & 500 & 570 & 730 & 990 \\
\hline 1651000 & $7 / 28 / 17$ & $11: 57$ & 5.92 & 4,320 & 5,260 & 560 & 630 & 790 & 1,400 \\
\hline 1651000 & $7 / 28 / 17$ & $12: 37$ & 7.47 & 7,670 & 7,670 & 790 & 740 & 1,100 & 2,200 \\
\hline 1651730 & $12 / 6 / 16$ & $21: 59$ & 1.96 & 216 & 223 & 110 & 109 & 120 & 170 \\
\hline 1651730 & $12 / 6 / 16$ & $22: 59$ & 2.27 & 165 & 162 & 93 & 92 & 88 & 130 \\
\hline 1651730 & $12 / 6 / 16$ & $23: 59$ & 2.62 & 115 & 116 & 74 & 76 & 69 & 100 \\
\hline 1651730 & $12 / 7 / 16$ & $0: 59$ & 2.63 & 85.6 & 86.8 & 64 & 64 & 55 & 80 \\
\hline 1651730 & $12 / 7 / 16$ & $1: 59$ & 2.28 & 77.2 & 68.3 & 56 & 57 & 47 & 67 \\
\hline 1651730 & $12 / 7 / 16$ & $2: 59$ & 1.81 & 40.6 & 43.4 & 53 & 52 & 39 & 53 \\
\hline 1651730 & $12 / 7 / 16$ & $3: 59$ & 1.33 & 25.2 & 24.8 & 49 & 49 & 37 & 42 \\
\hline 1651730 & $12 / 7 / 16$ & $4: 59$ & 0.90 & 15.3 & 14.9 & 47 & 47 & 36 & 34 \\
\hline 1651730 & $1 / 3 / 17$ & $14: 33$ & 2.32 & 251 & 241 & 222 & 216 & 220 & 290 \\
\hline 1651730 & $1 / 3 / 17$ & $15: 32$ & 1.81 & 171 & 169 & 177 & 181 & 200 & 220 \\
\hline 1651730 & $1 / 3 / 17$ & $16: 52$ & 1.37 & 136 & 138 & 153 & 153 & 240 & 180 \\
\hline 1651730 & $1 / 23 / 17$ & $12: 39$ & 1.69 & 197 & 198 & 117 & 154 & 140 & 210 \\
\hline 1651730 & $1 / 23 / 17$ & $13: 59$ & 2.50 & 214 & 212 & 212 & 156 & 160 & 220 \\
\hline 1651730 & $1 / 23 / 17$ & $14: 59$ & 4.40 & 506 & 516 & 364 & 360 & 550 & 560 \\
\hline
\end{tabular}


Table 3.1. Suspended-sediment data used to develop linear regression models. - Continued

[ft, feet; $\mathrm{ft}^{3} / \mathrm{s}$, cubic feet per second; FNU, Formazin Nephelometric Units; SSC, suspended-sediment concentration; mg/L, milligrams per liter; nr, not measured; average discharge and average turbidity are calculated from measurements beginning 15 minutes before through 15 minutes after the collection time reported for the suspended-sediment sample]

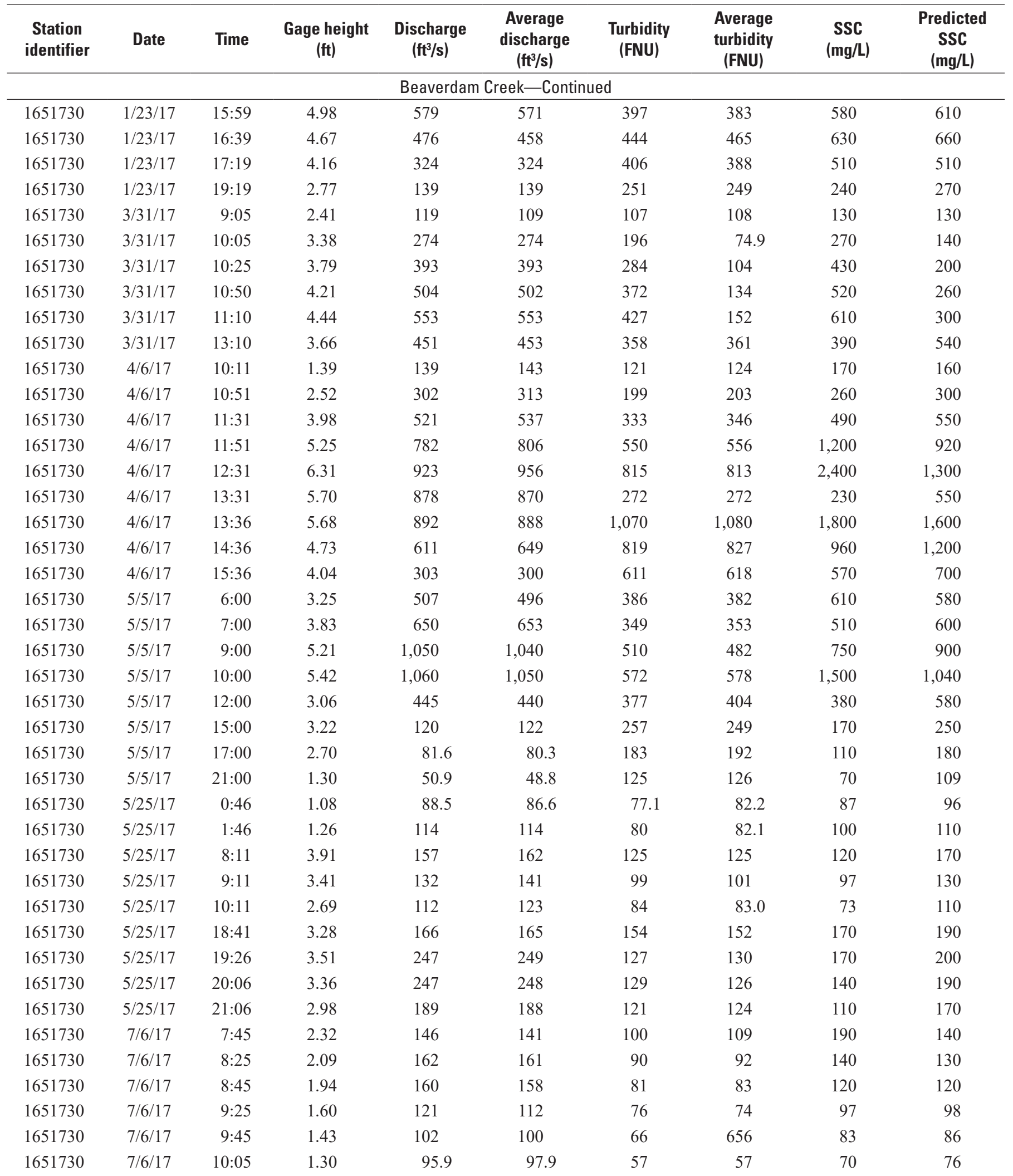


Table 3.1. Suspended-sediment data used to develop linear regression models. - Continued

[ft, feet; $\mathrm{ft}^{3} / \mathrm{s}$, cubic feet per second; FNU, Formazin Nephelometric Units; SSC, suspended-sediment concentration; mg/L, milligrams per liter; nr, not measured; average discharge and average turbidity are calculated from measurements beginning 15 minutes before through 15 minutes after the collection time reported for the suspended-sediment sample]

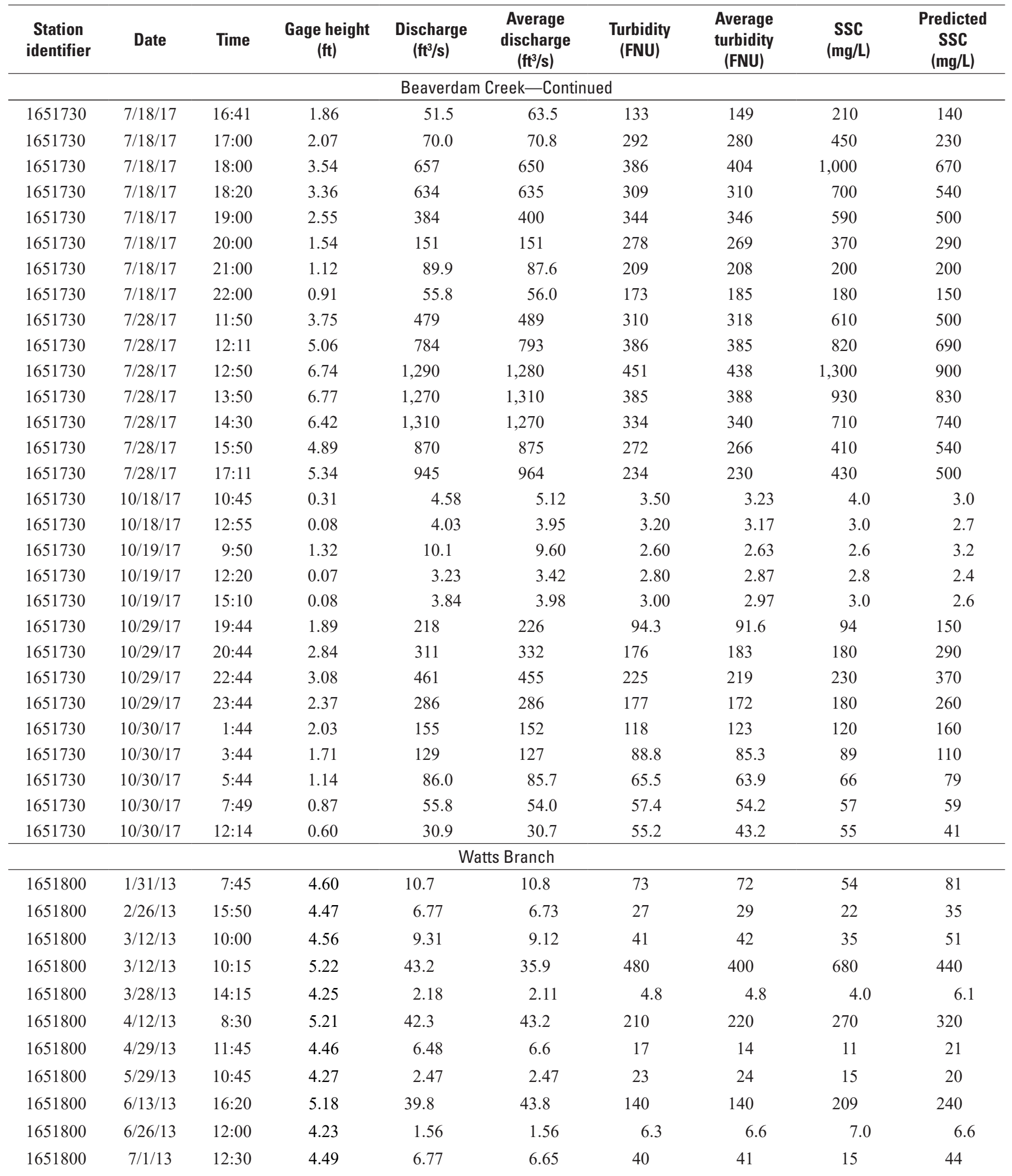


Table 3.1. Suspended-sediment data used to develop linear regression models. - Continued

[ft, feet; $\mathrm{ft}^{3} / \mathrm{s}$, cubic feet per second; FNU, Formazin Nephelometric Units; SSC, suspended-sediment concentration; mg/L, milligrams per liter; nr, not measured; average discharge and average turbidity are calculated from measurements beginning 15 minutes before through 15 minutes after the collection time reported for the suspended-sediment sample]

\begin{tabular}{|c|c|c|c|c|c|c|c|c|c|}
\hline $\begin{array}{l}\text { Station } \\
\text { identifier }\end{array}$ & Date & Time & $\begin{array}{c}\text { Gage height } \\
\text { (ft) }\end{array}$ & $\begin{array}{c}\text { Discharge } \\
\left(\mathrm{ft}^{3} / \mathrm{s}\right)\end{array}$ & $\begin{array}{c}\text { Average } \\
\text { discharge } \\
\left(\mathrm{ft}^{3} / \mathrm{s}\right)\end{array}$ & $\begin{array}{c}\text { Turbidity } \\
\text { (FNU) }\end{array}$ & $\begin{array}{c}\text { Average } \\
\text { turbidity } \\
\text { (FNU) }\end{array}$ & $\begin{array}{c}\text { SSC } \\
(\mathrm{mg} / \mathrm{L})\end{array}$ & $\begin{array}{c}\text { Predicted } \\
\text { SSC } \\
\text { (mg/L) }\end{array}$ \\
\hline \multicolumn{10}{|c|}{ Watts Branch-Continued } \\
\hline 1651800 & $7 / 25 / 13$ & $10: 15$ & 4.19 & 1.18 & 1.18 & 6.7 & 6.9 & 6.0 & 6.1 \\
\hline 1651800 & $6 / 13 / 13$ & $16: 20$ & 5.18 & 39.8 & 43.8 & 140 & 140 & 210 & 240 \\
\hline 1651800 & $6 / 26 / 13$ & $12: 00$ & 4.23 & 1.56 & 1.56 & 6.3 & 6.6 & 7.0 & 6.6 \\
\hline 1651800 & $10 / 28 / 13$ & $10: 45$ & 4.17 & 1.18 & 1.18 & 16 & 16 & 3.0 & 11 \\
\hline 1651800 & $11 / 1 / 13$ & $9: 30$ & 4.56 & 9.31 & 9.65 & 58 & 57 & 78 & 65 \\
\hline 1651800 & $11 / 26 / 13$ & $14: 15$ & 4.64 & 11.7 & 11.6 & 34 & 33 & 29 & 49 \\
\hline 1651800 & $12 / 22 / 13$ & $13: 00$ & 4.75 & 16.5 & 16.7 & 180 & 180 & 200 & 180 \\
\hline 1651800 & $2 / 5 / 14$ & $10: 45$ & 4.79 & 17.9 & 17.9 & 39 & 39 & 170 & 66 \\
\hline 1651800 & $2 / 25 / 14$ & $14: 00$ & 4.44 & 6.19 & 6.19 & 29 & 29 & 170 & 33 \\
\hline 1651800 & $3 / 27 / 14$ & $13: 15$ & 4.26 & 2.18 & 2.18 & 20 & 20 & 5.0 & 16 \\
\hline 1651800 & $3 / 29 / 14$ & $14: 30$ & 5.09 & 34.2 & 34.2 & 78 & 78 & 200 & 140 \\
\hline 1651800 & $3 / 30 / 14$ & $9: 45$ & 5.50 & 70.8 & 70.8 & 87 & 87 & 280 & 210 \\
\hline 1651800 & $4 / 15 / 14$ & $10: 45$ & 5.43 & 63.6 & 63.6 & 700 & 720 & 1,100 & 850 \\
\hline 1651800 & $4 / 29 / 14$ & $10: 30$ & 4.49 & 7.68 & 7.68 & 20 & 20 & 17 & 29 \\
\hline 1651800 & $4 / 30 / 14$ & $13: 30$ & 6.06 & 163 & 163 & 240 & 250 & 440 & 610 \\
\hline 1651800 & $5 / 28 / 14$ & $10: 15$ & 4.26 & 2.18 & 2.18 & 24 & 24 & 3.0 & 19 \\
\hline 1651800 & $10 / 22 / 14$ & $11: 05$ & 4.91 & 22.5 & 22.5 & 81 & 79 & 59 & 120 \\
\hline 1651800 & $10 / 28 / 14$ & $12: 00$ & 4.19 & 1.1 & 1.1 & 10 & 10 & 3.0 & 7.8 \\
\hline 1651800 & $11 / 17 / 14$ & $13: 30$ & 5.10 & 38.1 & 38.1 & 170 & 160 & 160 & 250 \\
\hline 1651800 & $11 / 25 / 14$ & $12: 45$ & 4.19 & 1.36 & 1.36 & 9.0 & 9.7 & 5.0 & 8.2 \\
\hline 1651800 & $12 / 23 / 14$ & 9:00 & 4.25 & 2.05 & 2.05 & 13 & 13 & 6.0 & 12 \\
\hline 1651800 & $12 / 24 / 14$ & $9: 45$ & 4.98 & 29.3 & 29.5 & 42 & 42 & 33 & 85 \\
\hline 1651800 & $1 / 26 / 15$ & $11: 15$ & 4.46 & 6.77 & 6.77 & 19 & 19 & 13 & 26 \\
\hline 1651800 & $2 / 24 / 15$ & 9:00 & 4.33 & 3.00 & 3.00 & 12 & 13 & 7.0 & 14 \\
\hline 1651800 & $3 / 24 / 15$ & $10: 00$ & 4.25 & 2.18 & 2.18 & 12 & 12 & 5.0 & 12 \\
\hline 1651800 & $5 / 26 / 15$ & $9: 15$ & 4.15 & 1.10 & 1.10 & 23 & 23 & 3.0 & 14 \\
\hline 1651800 & $6 / 20 / 15$ & $21: 00$ & 7.79 & 888 & 838 & 630 & 590 & 1,600 & 2,300 \\
\hline 1651800 & $6 / 20 / 15$ & $21: 10$ & 7.35 & 632 & 681 & 610 & 600 & 830 & 2,100 \\
\hline 1651800 & $6 / 23 / 15$ & $7: 45$ & 4.21 & 1.67 & 1.67 & 14 & 13 & 3.0 & 11 \\
\hline
\end{tabular}


Table 3.1. Suspended-sediment data used to develop linear regression models. - Continued

[ft, feet; $\mathrm{ft}^{3} / \mathrm{s}$, cubic feet per second; FNU, Formazin Nephelometric Units; SSC, suspended-sediment concentration; mg/L, milligrams per liter; nr, not measured; average discharge and average turbidity are calculated from measurements beginning 15 minutes before through 15 minutes after the collection time reported for the suspended-sediment sample]

\begin{tabular}{|c|c|c|c|c|c|c|c|c|c|}
\hline $\begin{array}{l}\text { Station } \\
\text { identifier }\end{array}$ & Date & Time & $\begin{array}{c}\text { Gage height } \\
\text { (ft) }\end{array}$ & $\begin{array}{c}\text { Discharge } \\
\left(\mathrm{ft}^{3} / \mathrm{s}\right)\end{array}$ & $\begin{array}{c}\text { Average } \\
\text { discharge } \\
\left(\mathrm{ft}^{3} / \mathrm{s}\right)\end{array}$ & $\begin{array}{c}\text { Turbidity } \\
\text { (FNU) }\end{array}$ & $\begin{array}{c}\text { Average } \\
\text { turbidity } \\
\text { (FNU) }\end{array}$ & $\begin{array}{l}\text { SSC } \\
\text { (mg/L) }\end{array}$ & $\begin{array}{c}\text { Predicted } \\
\text { SSC } \\
\text { (mg/L) }\end{array}$ \\
\hline \multicolumn{10}{|c|}{ Watts Branch-Continued } \\
\hline 1651800 & $7 / 28 / 15$ & $7: 30$ & 4.13 & 0.87 & 0.87 & 14 & 13 & 8.0 & 8.3 \\
\hline 1651800 & $7 / 30 / 15$ & $14: 30$ & 5.58 & 88 & 97.1 & 330 & 330 & 490 & 600 \\
\hline 1651800 & $9 / 29 / 15$ & $10: 00$ & 4.10 & 0.51 & 0.51 & 4.8 & 4.9 & 2.0 & 3.4 \\
\hline 1651800 & $10 / 28 / 15$ & $12: 20$ & 4.71 & 16.5 & 15.9 & 69 & 68 & 68 & 92 \\
\hline 1651800 & $10 / 29 / 15$ & $10: 50$ & 4.23 & 2.05 & 2.05 & 39 & 38 & 22 & 25 \\
\hline 1651800 & $11 / 24 / 15$ & $11: 25$ & 4.11 & 0.87 & 0.87 & 13 & 13 & 9.0 & 8.1 \\
\hline 1651800 & $12 / 1 / 15$ & $11: 15$ & 5.34 & 62.4 & 61.3 & 110 & 110 & 130 & 220 \\
\hline 1651800 & $2 / 3 / 16$ & $13: 35$ & 5.50 & 80.0 & 66.6 & 160 & 170 & 490 & 320 \\
\hline 1651800 & $2 / 16 / 16$ & $14: 15$ & 5.08 & 39.8 & 40.1 & 110 & 110 & 100 & 190 \\
\hline 1651800 & $2 / 25 / 16$ & $10: 20$ & 4.55 & 10.3 & 10.3 & 47 & 47 & 40 & 58 \\
\hline 1651800 & $3 / 29 / 16$ & $10: 30$ & 4.20 & 1.67 & 1.65 & 9.3 & 9.5 & 3.0 & 8.8 \\
\hline 1651800 & $4 / 7 / 16$ & $13: 10$ & 4.56 & 10.7 & 10.8 & 91 & 91 & 80 & 95 \\
\hline 1651800 & $5 / 6 / 16$ & 9:50 & 5.43 & 68.3 & 60.4 & 110 & 100 & 280 & 220 \\
\hline 1651800 & $5 / 25 / 16$ & $11: 30$ & 4.26 & 1.92 & 1.86 & 3.6 & 4.1 & 9.0 & 5.2 \\
\hline 1651800 & $6 / 28 / 16$ & $12: 25$ & 4.22 & 1.46 & 1.46 & 9.6 & 9.8 & 14 & 8.5 \\
\hline 1651800 & $7 / 29 / 16$ & $8: 25$ & 4.45 & 5.92 & 6.04 & 17 & 17 & 65 & 23 \\
\hline 1651800 & $11 / 30 / 16$ & $11: 36$ & 4.68 & 14.6 & 14.4 & 71 & 73 & 55 & 92 \\
\hline 1651800 & $1 / 23 / 17$ & $13: 50$ & 5.52 & 77.3 & 76.6 & 140 & 130 & 250 & 290 \\
\hline 1651800 & $2 / 28 / 17$ & $12: 40$ & 4.12 & 0.74 & 0.74 & 9.1 & 8.9 & 8.0 & 5.9 \\
\hline 1651800 & $3 / 28 / 17$ & $14: 00$ & 4.20 & 1.46 & 1.45 & 10 & 80 & 6.0 & 36 \\
\hline 1651800 & $3 / 31 / 17$ & $8: 40$ & 4.94 & 26.1 & 25.6 & 81 & 78 & 89 & 120 \\
\hline 1651800 & $3 / 31 / 17$ & $9: 16$ & 5.38 & 61.3 & 61.0 & 170 & 160 & 310 & 300 \\
\hline 1651800 & $3 / 31 / 17$ & $10: 28$ & 5.79 & 114 & 111 & 190 & 190 & 360 & 430 \\
\hline 1651800 & $3 / 31 / 17$ & $11: 04$ & 5.54 & 80 & 79.4 & 170 & 170 & 250 & 350 \\
\hline 1651800 & $3 / 31 / 17$ & $11: 12$ & 5.49 & 73.4 & 73.0 & 170 & 170 & 250 & 340 \\
\hline 1651800 & $3 / 31 / 17$ & $11: 16$ & 5.47 & 70.8 & 71.2 & 170 & 170 & 230 & 340 \\
\hline 1651800 & $3 / 31 / 17$ & $11: 52$ & 5.38 & 61.3 & 61.8 & 140 & 140 & 190 & 280 \\
\hline 1651800 & $4 / 25 / 17$ & $13: 00$ & 4.38 & 4.6 & 4.64 & 10 & 12 & 7.0 & 16 \\
\hline 1651800 & $5 / 5 / 17$ & $5: 16$ & 5.47 & 71.5 & 71.5 & 210 & 220 & 43 & 390 \\
\hline
\end{tabular}


Table 3.1. Suspended-sediment data used to develop linear regression models. - Continued

[ft, feet; $\mathrm{ft}^{3} / \mathrm{s}$, cubic feet per second; FNU, Formazin Nephelometric Units; SSC, suspended-sediment concentration; mg/L, milligrams per liter; nr, not measured; average discharge and average turbidity are calculated from measurements beginning 15 minutes before through 15 minutes after the collection time reported for the suspended-sediment sample]

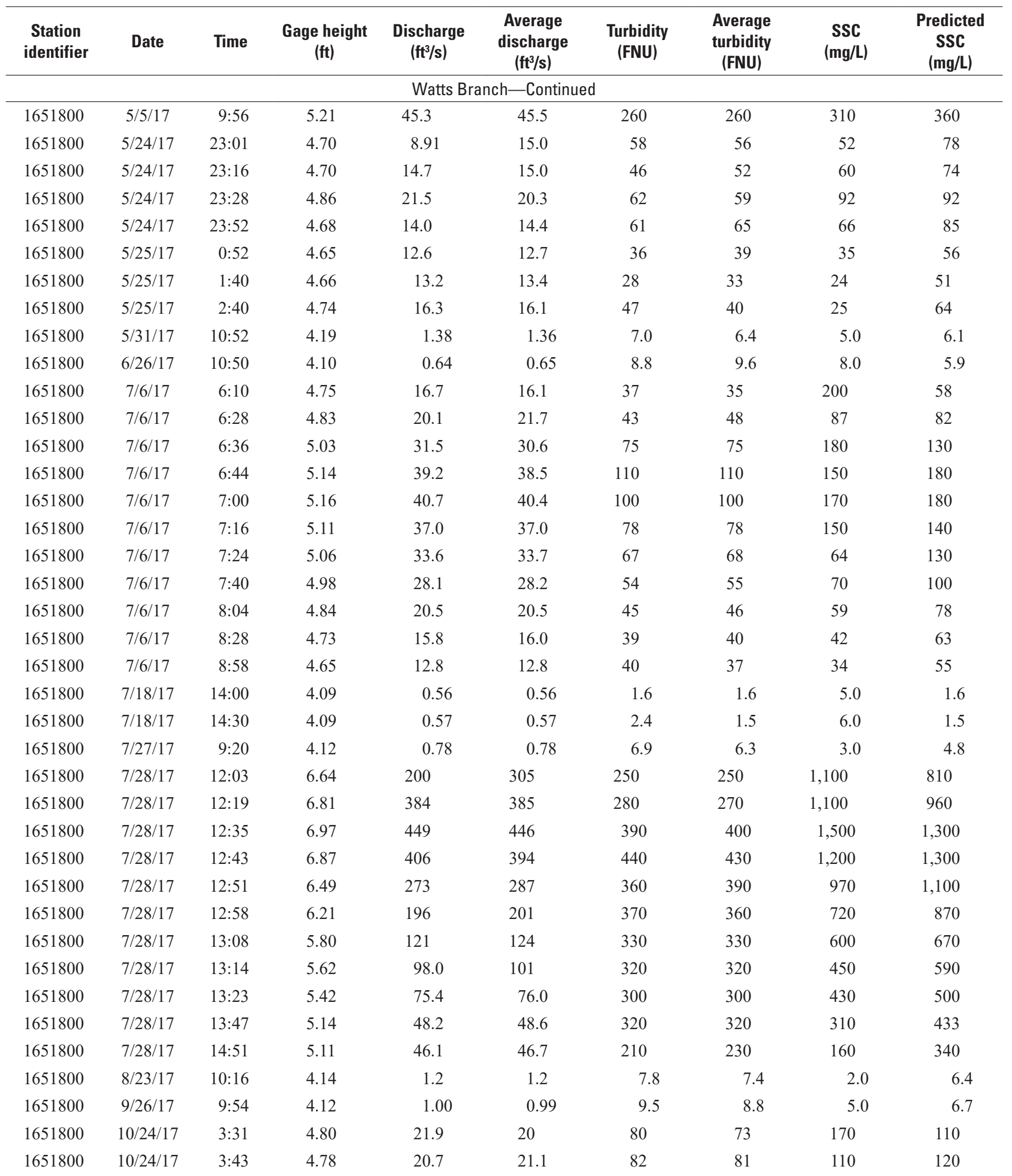


Table 3.1. Suspended-sediment data used to develop linear regression models. - Continued

[ft, feet; $\mathrm{ft}^{3} / \mathrm{s}$, cubic feet per second; FNU, Formazin Nephelometric Units; SSC, suspended-sediment concentration; mg/L, milligrams per liter; nr, not measured; average discharge and average turbidity are calculated from measurements beginning 15 minutes before through 15 minutes after the collection time reported for the suspended-sediment sample]

\begin{tabular}{|c|c|c|c|c|c|c|c|c|c|}
\hline $\begin{array}{l}\text { Station } \\
\text { identifier }\end{array}$ & Date & Time & $\begin{array}{c}\text { Gage height } \\
\text { (ft) }\end{array}$ & $\begin{array}{c}\text { Discharge } \\
\left(\mathrm{ft}^{3} / \mathrm{s}\right)\end{array}$ & $\begin{array}{c}\text { Average } \\
\text { discharge } \\
\left(\mathrm{ft}^{3} / \mathrm{s}\right)\end{array}$ & $\begin{array}{c}\text { Turbidity } \\
\text { (FNU) }\end{array}$ & $\begin{array}{c}\text { Average } \\
\text { turbidity } \\
\text { (FNU) }\end{array}$ & $\begin{array}{c}\text { SSC } \\
(\mathrm{mg} / \mathrm{L})\end{array}$ & $\begin{array}{c}\text { Predicted } \\
\text { SSC } \\
\text { (mg/L) }\end{array}$ \\
\hline \multicolumn{10}{|c|}{ Watts Branch-Continued } \\
\hline 1651800 & $10 / 24 / 17$ & $3: 55$ & 4.86 & 25.6 & 24.6 & 85 & 85 & 120 & 130 \\
\hline 1651800 & $10 / 24 / 17$ & $4: 07$ & 4.85 & 24.8 & 24.7 & 97 & 100 & 120 & 150 \\
\hline 1651800 & $10 / 24 / 17$ & $4: 19$ & 4.78 & 20.6 & 20.8 & 120 & 110 & 120 & 150 \\
\hline 1651800 & $10 / 24 / 17$ & $4: 43$ & 4.67 & 15.2 & 15.4 & 100 & 99 & 100 & 120 \\
\hline 1651800 & $10 / 24 / 17$ & $4: 55$ & 4.64 & 13.8 & 13.9 & 79 & 79 & 79 & 96 \\
\hline 1651800 & $10 / 29 / 17$ & $20: 55$ & 5.31 & 64.2 & 61.1 & 86 & 95 & 180 & 210 \\
\hline 1651800 & $10 / 29 / 17$ & $21: 19$ & 5.80 & 121 & 114 & 230 & 200 & 560 & 460 \\
\hline 1651800 & $11 / 7 / 17$ & $13: 32$ & 4.86 & 25.3 & 25.6 & 31 & 34 & 51 & 70 \\
\hline 1651800 & $11 / 16 / 17$ & $11: 10$ & 4.21 & 1.2 & 1.24 & 3.9 & 4.0 & 4.0 & 4.3 \\
\hline 1651800 & $11 / 16 / 17$ & $13: 00$ & 4.22 & 1.21 & 1.21 & 3.1 & 3.1 & 5.0 & 3.5 \\
\hline 1651800 & $11 / 16 / 17$ & $14: 30$ & 4.20 & 1.11 & 1.12 & 3.0 & 3.0 & 3.0 & 3.4 \\
\hline 1651800 & $11 / 29 / 17$ & $12: 10$ & 4.28 & 1.63 & 1.66 & 6.2 & 6.2 & 2.0 & 6.6 \\
\hline 1651800 & $12 / 21 / 17$ & $11: 10$ & 4.31 & 1.33 & 1.34 & 6.9 & 9.1 & 3.0 & 7.8 \\
\hline 1651800 & $1 / 12 / 18$ & $1: 00$ & 4.51 & 9.53 & $\mathrm{nr}$ & 75 & $\mathrm{nr}$ & 81 & 78 \\
\hline 1651800 & $1 / 25 / 18$ & $3: 16$ & 4.38 & 5.34 & $\mathrm{nr}$ & 52 & $\mathrm{nr}$ & 67 & 47 \\
\hline \multicolumn{10}{|c|}{ Hickey Run } \\
\hline 1651770 & $6 / 7 / 13$ & $10: 15$ & 2.88 & 17.4 & 17.7 & 45 & 44 & 35 & 83 \\
\hline 1651770 & $6 / 13 / 13$ & $15: 20$ & 3.44 & 48.3 & 44.7 & 190 & 210 & 280 & 290 \\
\hline 1651770 & $6 / 28 / 13$ & $10: 15$ & 1.83 & 0.51 & 0.57 & 15 & 13 & 7.0 & 15 \\
\hline 1651770 & $7 / 1 / 13$ & $11: 45$ & 2.5 & 8.45 & 8.62 & 55 & 55 & 46 & 80 \\
\hline 1651770 & $7 / 25 / 13$ & $11: 15$ & 1.8 & 0.39 & 0.39 & 8.8 & 8.9 & 6.0 & 11 \\
\hline 1651770 & $8 / 27 / 13$ & $12: 15$ & 1.76 & 0.21 & 0.21 & 4.5 & 4.5 & 5.0 & 5.9 \\
\hline 1651770 & $9 / 27 / 13$ & $11: 45$ & 2.00 & 1.42 & 1.38 & 5.5 & 5.5 & 4.0 & 11 \\
\hline 1651770 & $10 / 28 / 13$ & $11: 45$ & 1.78 & 0.22 & 0.22 & 5.8 & 5.4 & 2.0 & 6.7 \\
\hline 1651770 & $11 / 1 / 13$ & $8: 45$ & 2.98 & 22.6 & 17.0 & 430 & 390 & 520 & 340 \\
\hline 1651770 & $11 / 26 / 13$ & $15: 00$ & 2.43 & 6.65 & 7.00 & 52 & 53 & 41 & 73 \\
\hline 1651770 & $12 / 22 / 13$ & $13: 30$ & 2.07 & 1.86 & 1.82 & 56 & 53 & 180 & 52 \\
\hline 1651770 & $12 / 23 / 13$ & $8: 45$ & 2.46 & 7.17 & 7.07 & 53 & 53 & 130 & 74 \\
\hline 1651770 & $12 / 30 / 13$ & $12: 45$ & 2.01 & 1.34 & 1.34 & 42 & 41 & 76 & 40 \\
\hline
\end{tabular}


Table 3.1. Suspended-sediment data used to develop linear regression models. - Continued

[ft, feet; $\mathrm{ft}^{3} / \mathrm{s}$, cubic feet per second; FNU, Formazin Nephelometric Units; SSC, suspended-sediment concentration; mg/L, milligrams per liter; nr, not measured; average discharge and average turbidity are calculated from measurements beginning 15 minutes before through 15 minutes after the collection time reported for the suspended-sediment sample]

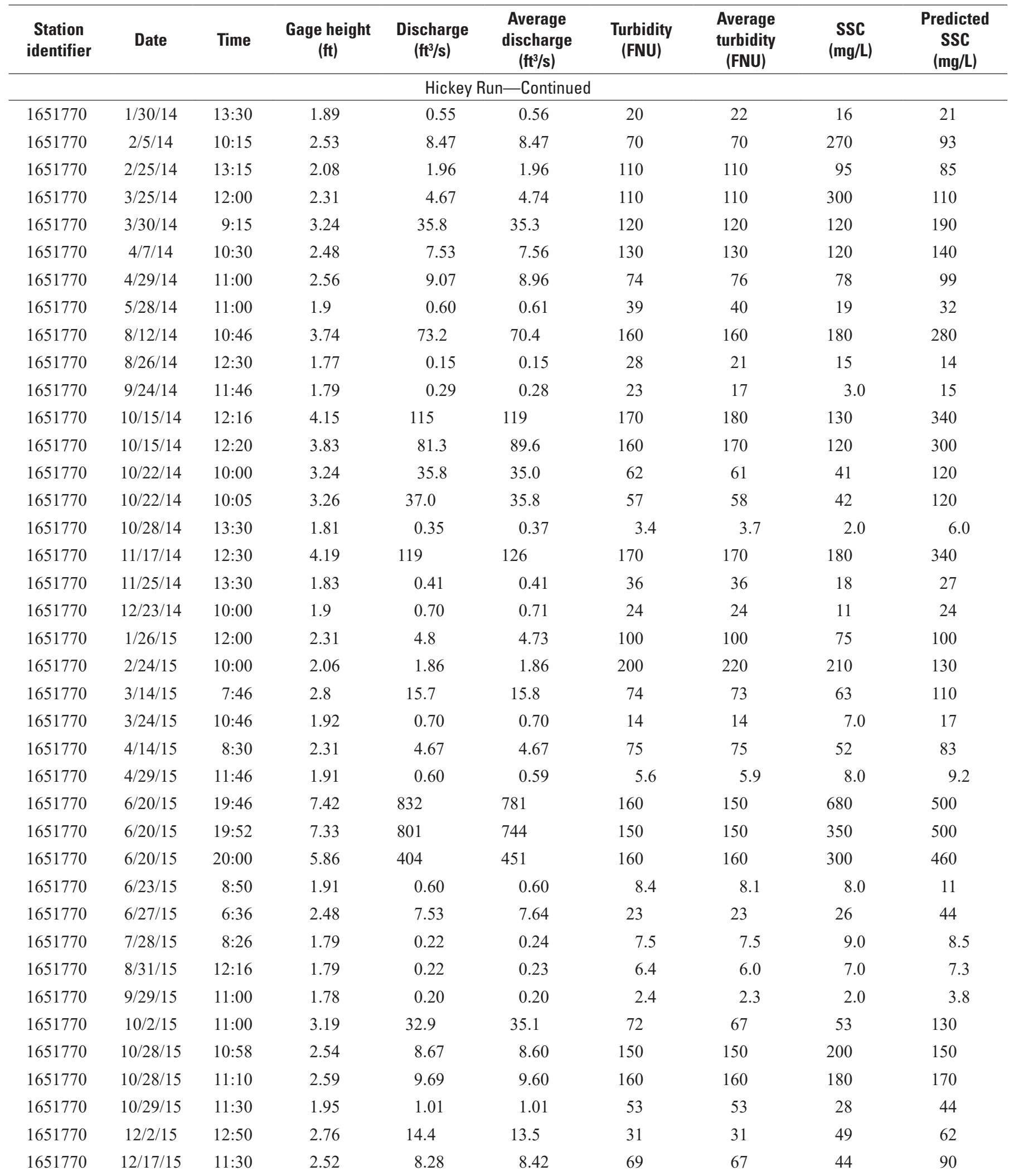


Table 3.1. Suspended-sediment data used to develop linear regression models. - Continued

[ft, feet; $\mathrm{ft}^{3} / \mathrm{s}$, cubic feet per second; FNU, Formazin Nephelometric Units; SSC, suspended-sediment concentration; mg/L, milligrams per liter; nr, not measured; average discharge and average turbidity are calculated from measurements beginning 15 minutes before through 15 minutes after the collection time reported for the suspended-sediment sample]

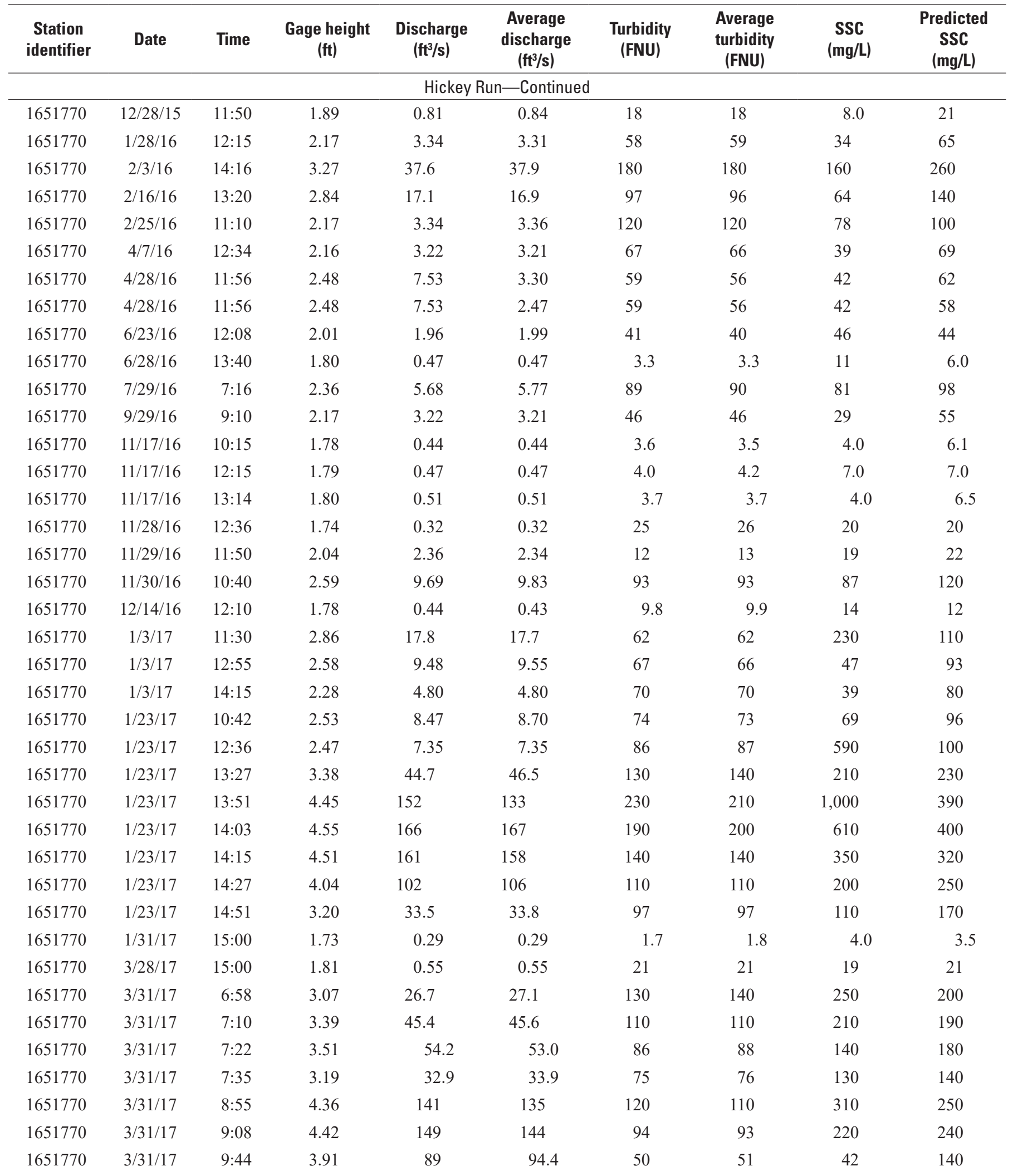


Table 3.1. Suspended-sediment data used to develop linear regression models. - Continued

[ft, feet; $\mathrm{ft}^{3} / \mathrm{s}$, cubic feet per second; FNU, Formazin Nephelometric Units; SSC, suspended-sediment concentration; mg/L, milligrams per liter; nr, not measured; average discharge and average turbidity are calculated from measurements beginning 15 minutes before through 15 minutes after the collection time reported for the suspended-sediment sample]

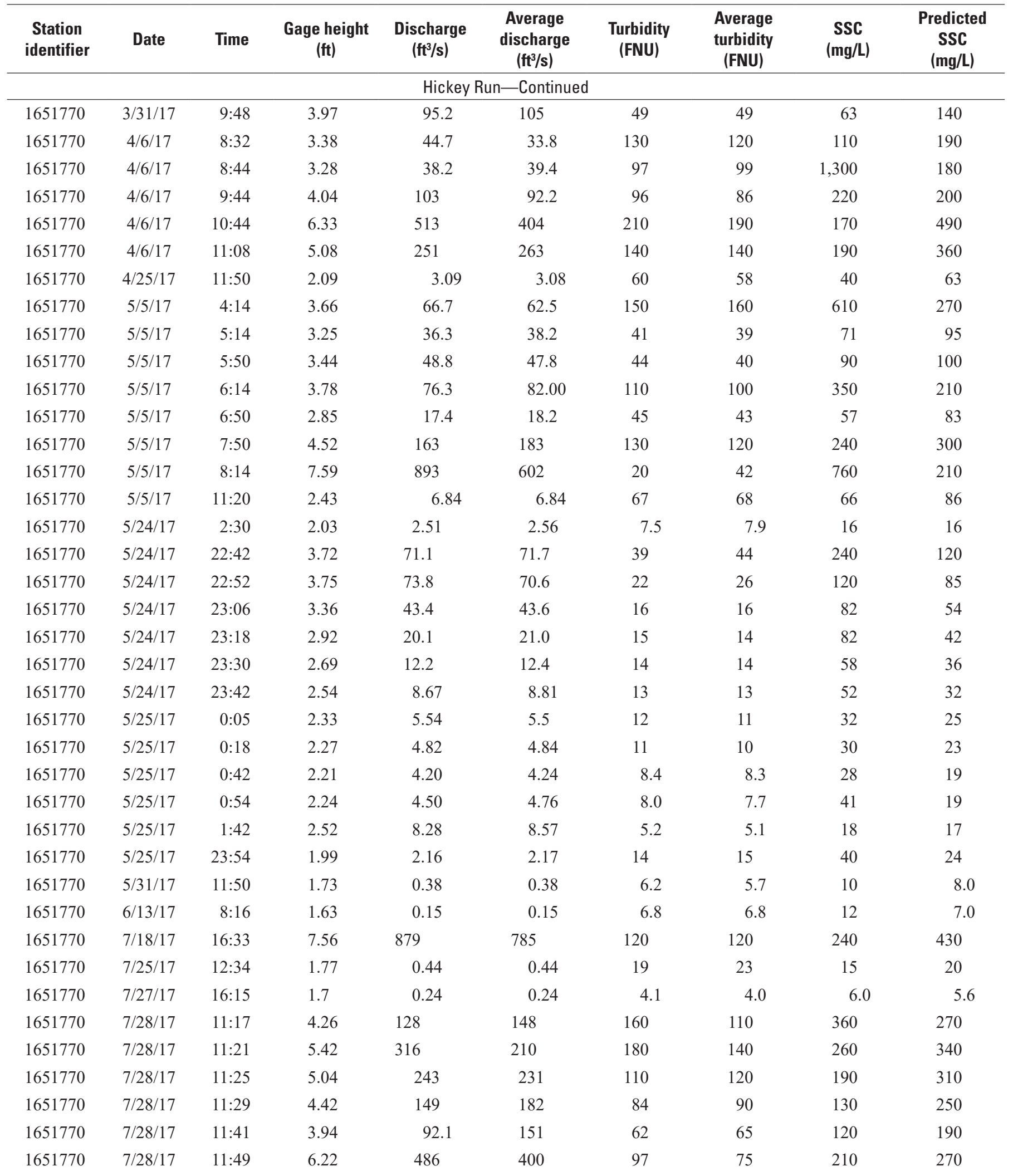


Table 3.1. Suspended-sediment data used to develop linear regression models. - Continued

[ft, feet; $\mathrm{ft}^{3} / \mathrm{s}$, cubic feet per second; FNU, Formazin Nephelometric Units; SSC, suspended-sediment concentration; mg/L, milligrams per liter; nr, not measured; average discharge and average turbidity are calculated from measurements beginning 15 minutes before through 15 minutes after the collection time reported for the suspended-sediment sample]

\begin{tabular}{cccccccccc}
\hline $\begin{array}{c}\text { Station } \\
\text { identifier }\end{array}$ & Date & Time & $\begin{array}{c}\text { Gage height } \\
\text { (ft) }\end{array}$ & $\begin{array}{c}\text { Discharge } \\
\text { (ft/s) } / \mathbf{s})\end{array}$ & $\begin{array}{c}\text { Average } \\
\text { discharge } \\
\text { (ft/s) }\end{array}$ & $\begin{array}{c}\text { Turbidity } \\
\text { (FNU) }\end{array}$ & $\begin{array}{c}\text { Average } \\
\text { turbidity } \\
\text { (FNU) }\end{array}$ & $\begin{array}{c}\text { SSC } \\
\text { (mg/L) }\end{array}$ & $\begin{array}{c}\text { Predicted } \\
\text { SSC } \\
\text { (mg/L) }\end{array}$ \\
\hline & & & \multicolumn{7}{c}{ Hickey Run-Continued } \\
\hline 1651770 & $7 / 28 / 17$ & $11: 53$ & 6.64 & 592 & 472 & 77 & 83 & 200 & 300 \\
1651770 & $7 / 28 / 17$ & $12: 09$ & 4.39 & 145 & 175 & 99 & 97 & 140 & 260 \\
1651770 & $7 / 28 / 17$ & $12: 17$ & 4.87 & 214 & 212 & 82 & 80 & 110 & 240 \\
1651770 & $7 / 28 / 17$ & $12: 21$ & 5.11 & 256 & 241 & 63 & 69 & 88 & 220 \\
1651770 & $7 / 28 / 17$ & $12: 41$ & 3.75 & 73.8 & 78.9 & 90 & 86 & 100 & 190 \\
1651770 & $9 / 5 / 17$ & $13: 20$ & 1.80 & 0.59 & 0.60 & 1.9 & 1.8 & 3.0 & 4.3 \\
1651770 & $9 / 26 / 17$ & $12: 48$ & 1.67 & 0.20 & 0.21 & 2.3 & 2.3 & 2.0 & 3.8 \\
1651770 & $11 / 1 / 17$ & $10: 40$ & 1.68 & 0.13 & 0.13 & 12 & 12 & 8.0 & 10 \\
1651770 & $11 / 7 / 17$ & $11: 44$ & 2.33 & 5.28 & 5.63 & 31 & 40 & 62 & 58 \\
1651770 & $11 / 7 / 17$ & $11: 48$ & 2.60 & 9.98 & 9.02 & 61 & 55 & 71 & 80 \\
1651770 & $11 / 7 / 17$ & $11: 56$ & 2.71 & 12.9 & 12.2 & 77 & 75 & 100 & 110 \\
1651770 & $11 / 7 / 17$ & $12: 04$ & 2.62 & 10.4 & 10.6 & 77 & 76 & 67 & 100 \\
1651770 & $11 / 7 / 17$ & $12: 16$ & 2.52 & 8.34 & 9.11 & 70 & 71 & 49 & 95 \\
1651770 & $11 / 7 / 17$ & $12: 32$ & 3.00 & 23.6 & 22.8 & 75 & 74 & 79 & 130 \\
1651770 & $11 / 7 / 17$ & $12: 34$ & 3.03 & 25.0 & 24.4 & 75 & 75 & 75 & 130 \\
1651770 & $11 / 7 / 17$ & $12: 40$ & 3.09 & 27.9 & 26.8 & 78 & 77 & 89 & 130 \\
1651770 & $11 / 7 / 17$ & $12: 56$ & 2.97 & 22.3 & 23.3 & 74 & 72 & 62 & 120 \\
1651770 & $11 / 29 / 17$ & $13: 10$ & 1.78 & 0.24 & 0.25 & 2.2 & 3.5 & 3.0 & 5.2 \\
1651770 & $12 / 21 / 17$ & $10: 26$ & 1.68 & 0.14 & 0.14 & 3.8 & 3.8 & 6.0 & 4.7 \\
1651770 & $1 / 12 / 18$ & $9: 48$ & 1.87 & 0.92 & 0.92 & 59 & 60 & 44 & 47 \\
1651770 & $1 / 25 / 18$ & $12: 26$ & 1.65 & 0.16 & 0.16 & 4.3 & 4.2 & 7.0 & 5.2 \\
\hline
\end{tabular}

\section{Reference Cited}

Wilson, T.P., 2019, Discharge and sediment data for selected tributaries to the Anacostia River, Washington, D.C., 2003-18:

U.S. Geological Survey data release, https://doi.org/10.5066/P9RUZSMV. 
For additional information, contact:

Director, MD-DE-DC Water Science Center U.S. Geological Survey

5522 Research Park Drive

Baltimore, MD 21228

or visit our website at: https://www.usgs.gov/centers/md-de-dc-water/

Publishing support provided by the West Trenton Publishing Service Center 


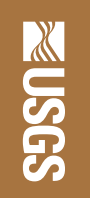

$\sum_{\substack{\mathrm{D} \\ \mathrm{O}}}$

i

赵

䨔

言

$\frac{2}{\frac{9}{2}}$

ㅎ.

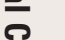

을

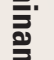

5:

$\Xi$

ISSN 2328-0328 (online) 